\title{
Acquisition and Inversion of Dispersive Seismic Waves in Shallow Marine Environments
}

\author{
Dissertation \\ zur Erlangung des Doktorgrades \\ der Mathematisch-Naturwissenschaftlichen Fakultät \\ der Christian-Albrechts-Universität \\ zu Kiel
}

vorgelegt von

Gerald Klein

Kiel

April 2003 
Referent:

Korreferent:

Tag der mündlichen Prüfung:

Zum Druck genehmigt:
Prof. Dr. Wolfgang Rabbel Prof. Dr. Bernd Milkereit

10. Juni 2003

10. Juli 2003 


\section{Zusammenfassung}

Die Kenntnis der elastischen Eigenschaften mariner Sedimente, insbesondere die Scherwellengeschwindigkeit, ist eine wichtige Information für die Untersuchung der Stabilität von Meeresböden. Dies ist insbesondere für geotechnische Anwendungen im flachmarinen Bereich von Bedeutung, aber auch für die Untersuchung von Hangstabilitäten der Kontinentalränder und für die Gashydratforschung von Interesse.

Seismische Experimente in der Ostsee sowie die Analyse eines Datensatzes aus der Nordsibirischen Laptevsee konnten zeigen, dass dispersive seismische Wellenfelder mit Hilfe einer nahe der Meeresoberfläche geschleppten seismischen Quelle (Airgun) angeregt werden können. Sowohl dispersive Grenzflächenwellen als auch geführte Wasserwellen konnten einerseits mit einem stationären Empfänger auf dem Meeresboden, andererseits mit einer nahe dem Meeresboden geschleppten Hydrophonkette registriert werden. Dabei musste festgestellt werden, dass die Meßbarkeit beider Wellentypen stark von der zu untersuchenden Untergrundstruktur abhängig ist. Die Dispersion der sich am Meeresboden ausbreitenden Grenzflächenwelle (Scholte-Welle), wird besonders durch die Tiefenverteilung der Scherwellengeschwindigkeit des Untergrundes bestimmt. Dagegen reagieren die Dispersionseigenschaften der geführten Wasserwellen auch stark auf Änderungen der Kompressionswellengeschwindigkeiten und der Dichte.

Die seismischen Eigenschaften in Form von den seismischen Ausbreitungsgeschwindigkeiten der Scher- und Kompressionwellenenergie sowie der Dichte werden mit Hilfe einer iterativen Least-Squares-Inversion aus gemessenen Wellenfeldspektren bestimmt. Diese werden durch eine zweidimensionale Wellenfeldtransformation aus den mit ausreichend engem Abstand vorhandenen Seismogrammfamilien erzeugt. Die Anwendung der Inversion auf Wellenfeldspektren hat gegenüber einer Inversion von Wellenformen bzw. Seismogrammen deutliche Vorteile in Hinblick auf die Linearisierbarkeit und Stabilität der Inversion. Andererseits ist sie einfacheren Inversionsverfahren zur Anpassung von Dispersionskurven überlegen, da hier die Information des kompletten Wellenfelds inklusive der Amplituden und Phasenspektren berücksichtigt wird und somit keine Zuordnung der Moden nötig ist.

Die Anwendung der 2-D Wellenfeldtransformation bedingt die Anforderung der engen räumlichen Abtastung des Wellenfeldes und damit verbunden Grenzen der Durchführbarkeit der Messung oder des Auflösungsvermögens der Inversion. Diese sind jedoch für beide Wellentypen unterschiedlich zu betrachten. Die Registrierung und Auflösung von dispersiven Grenzflächenwellen, insbesondere der Scholte-Welle, ist in weichen schlickhaltigen Meeresböden mit Scherwellengeschwindigkeiten unter $50 \mathrm{~m} / \mathrm{s}$ sehr problematisch, da hier das Abtastkriterium mit den bisher verfügbaren Meßgeräten nicht erfüllt werden kann. Jedoch konnte die Scherwellengeschwindigkeit fester Untergründe mit Hilfe von Inversionsverfahren bestimmt werden, sowie Aufschluß über die laterale Variation der Schichtparameter gewonnen werden. 
Die Auswertung von dispersiven geführten Wellen erfordert die Registrierung des Wellenfeldes über große Distanzen. Auswertbare Daten wurden mit Auslagen der Registrierung ab ca. $800 \mathrm{~m}$ erreicht. Wie allgemein angenommen, werden die Dispersionseigenschaften dieses Wellentyps nur bei Vorhandensein eines harten Untergrunds, bzw. eines starken Kontrast der elastischen Eigenschaften, meßbar. Es konnte am Beispiel eines Datensatzes aus dem Arkonabecken (Ostsee) gezeigt werden, dass hier eine ca. 10 - 12 m mächtige Schicht weicher Sedimente die Registrierung und Auswertung der akustischen Moden nicht behindern. Vielmehr konnte auch eine grobe Abschätzung der Scherwellengeschwindigkeit erfolgen, auch wenn die Sensitivität und dadurch auch die Auflösung in den darunterliegenden harten Untergrundschichten höher war als für den weichen Meeresboden.

Das geschleppte Meßverfahren konnte laterale Variationen der Dispersionseigenschaften von Grenzflächenwellen auflösen. Die quantitative Aussagekraft war durch systembedingte Ausfälle beeinträchtigt, zeigte jedoch, dass die Anwendbarkeit der Methode mit einem langen, tiefgeschleppten Meßsystem erfolgversprechend ist. Aufgrund der Anforderungen an die Akquisition wird die Anwendbarkeit jedoch in Meßgebieten mit weichen Untergründen sowie in kleinräumigen Wassergebieten (z.B. Hafenanlagen) problematisch sein. Die Scherwellengeschwindigkeit für harte Meeresböden kann mit Hilfe der Inversionsverfahren ermittelt werden. Die Ergebnisse der geschleppten Meßanordnung zeigen, dass diese Methode für die Identifizierung von Bereichen festen Untergrundes in großräumigem Untersuchungsgebieten mit gutem Meßfortschritt denkbar und nützlich wäre. 


\section{Abstract}

The knowledge of the shear wave velocity structure of shallow marine sediments is an important information for the assessment of sediment stability. This is of interest for marine geotechnical applications as well as investigations of sea-floor stability of continental shelves and margins and in gashydrate research. The derivation of seismic velocities with special interest on the shear wave velocity from the analysis and inversion of dispersive seismic waves is investigated in this work.

Dispersive waves are excited by surface towed airguns and the acquisition was achieved with two different configurations. Firstly, the stationary-receiver method comprises of an ocean bottom seismometer station (OBS) and excitation with a surface towed airgun. Secondly, a towed-acquisition system includes a streamer towed at ten meters above the sea bed and excitation with an airgun. Two different wave types could be observed with either of the two acquisition systems. Interface waves, namely of the Scholte wave type, propagating along the sea floor and guided waves in the water column, denoted as acoustic guided waves despite of their sediment interaction at the sea bed. The both wave types were observed in certain environments indicating the limitations in the acquisition of these wave types depending on the sediment properties. These limitations as well as the difference in dispersion sensitivity to variations of the seismic properties give rise for separate treatment of both wave types. While the dispersion sensitivity of Scholte waves as well as other interface waves is dominant for shear wave velocity variations, the variation of the dispersion of acoustic guided waves is affected by variations of compressional and shear wave velocity as well as density to a smaller extend. The difference in velocity and frequency range of interest for both wave types, i.e. the ranges of most variations in the dispersion characteristics, also affect the required acquisition configuration and parameters. While a long offset recording (exceeding $800 \mathrm{~m}$ ) is required for acoustic guided waves, the fulfillment of the spatial sample criteria sometimes limits the feasibility to adequately acquire the dispersive wavefield. This is most dominant for the Scholte wave measurements in very soft marine sediments.

The derivation of the shear wave velocity structure was investigated at three study areas in the Kiel Bay and Arkona Basin of the Baltic Sea and from a data set of the Laptev Sea in North Siberia. The derivation of shear wave velocites from the dispersion of Scholte waves has been successfully applied in several locations. Limitations for the acquisition of Scholte waves remain for very soft marine sediments with shear wave velocities lower than $50 \mathrm{~m} / \mathrm{s}$ due to the required dense spatial sampling and the low frequency range of interest for these conditions. However, for shallow marine sediments exceeding shear wave velocities of 60 $\mathrm{m} / \mathrm{s}$ sufficient Scholte wave energy could be excited with a surface towed airgun and the shear wave velocity was successfully inverted from dispersive seismic wavefields recorded at or near the sea floor.

Acoustic guided waves recorded with a long streamer in the Laptev Sea and with a sta- 
tionary receiver in the central Arkona Basin could be used to infer sub-surface properties including the shear wave velocity by inversion of the full wavefield spectra. Since this wave type is sensitive to compressional wave velocity and density as well as the shear wave velocity, the ambiguities have to be compensated by additional constraints. However, shear wave velocity information was obtained from this wave type at the central Arkona Basin where no seismic interface wave could be recorded. This wave type is generally known to require a hard contrast at the sea floor, yet the acoustic guided wave could be observed and analyzed in the central Arkona Basin despite of the presence of a 10 to $12 \mathrm{~m}$ thick layer of soft sediments above stiffer materials. However, the sensitivity of the shear wave velocity information is low for the soft sediment layer in this specific data set.

In order to infer sub-bottom properties a linear-least-squares inversion of the wavefield transformed data is applied in the frequency-slowness domain. The inversion of the wavefield spectra is advantageous in terms of stability and linearisation of the inversion compared to full waveform inversion of seismograms. The method was adapted for the application to marine seismic data and enables to invert the full complex spectral coefficients of the transformed seismic wavefield. Therefore all the information contained in the seismic data but not easily extractable in form of a dispersion curve can be included in the inversion. However, in practice it is sometimes favorable to apply certain normalization and weighting to the data and synthetics in order to suppress noise and artifact energy.

With the towed acquisition system lateral variations of the dispersive interface wave could be resolved. While the interpretation was complicated by acquisition problems of the streamer, the general applicability of the method to infer lateral variability of variation of the shear wave velocity with depth with a towed acquisition system. This could be used to effectively determine areas of rigid sea-floor and sub sear-floor properties. 


\section{Contents}

1 Introduction $\quad 11$

I Theory and Methods $\quad 15$

2 Dispersive Waves in Marine Sediments 17

2.1 Marine Sediments . . . . . . . . . . . . . . . . . . . 18

2.2 Seismic wave propagation . . . . . . . . . . . . . 20

2.3 Poroelastic and viscoelastic material properties . . . . . . . . . . 23

2.4 Lamb's problem . . . . . . . . . . . . . . . . . . . . 24

2.5 Scholte waves . . . . . . . . . . . . . . . . 26

2.6 Dispersive waves in marine conditions . . . . . . . . . . . . 28

2.6.1 Dispersive waves in a stratified medium . . . . . . . . . . 28

2.6.2 Wave guides and leaky modes . . . . . . . . . . . . . . 30

2.6.3 Dissipative and other dispersion effects . . . . . . . . . . . 33

3 Analysis of Dispersive Seismic Waves 37

3.1 Modeling of Seismic Dispersion . . . . . . . . . . . . . . . . . 38

3.2 Analysis of Dispersive Seismic Wavefields . . . . . . . . . . . . . . . . 39

3.3 Multi-channel Dispersion Analysis . . . . . . . . . . . . . . . . . . 40

3.3.1 Fourier Transform and Slant-stacking Methods . . . . . . . . . . . . 41

3.3.2 Modified Fourier-Bessel Transform . . . . . . . . . . . . . . . . . 44

4 Acquisition of Dispersive Wavefields 47

4.1 Determination of the Acquisition Parameters . . . . . . . . . . . . . . . . 48

4.1.1 Determining the Frequency Range of Interest . . . . . . . . . . . . . 48

4.1.2 Energy Distribution with Depth of Scholte Waves . . . . . . . . . . 50

4.2 Excitation and Registration of Interface Waves . . . . . . . . . . . . . . . . . . . . . . . . 50

4.2.1 Seismic Sources at the Sea bed . . . . . . . . . . . . . 50 50 
4.2.2 Seismic Receivers at the Sea Bed . . . . . . . . . . . . . . 52

4.3 Seismogram Gathers in Different Acquisition Geometries . . . . . . . . . . . 53

4.3.1 Obeying the 2-D Sampling Theorem in Time and Space . . . . . . 53

4.3.2 Common-Receiver-Gather $(\mathrm{OBS} / \mathrm{H}) \ldots \ldots \ldots . \ldots 54$

4.3.3 Common-Shot-Gather $(\mathrm{OBC} /$ Streamer $) \ldots \ldots \ldots 56$

5 Inferring Sediment Properties from Measured Dispersive Wavefield Spectra 59

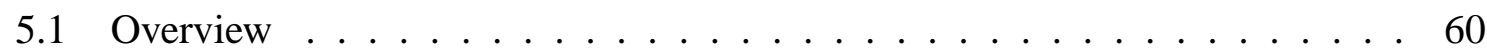

5.2 Modified Dispersion Curve Inversion . . . . . . . . . . . . . . . . . 61

5.3 Inversion of the full wavefield spectra . . . . . . . . . . . . . . . 62

5.4 Adaption of the full wavefield inversion for marine seismic data $\ldots . . . .68$

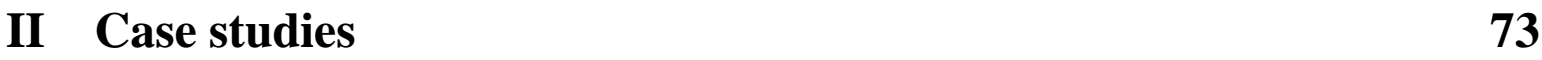

6 Scholte Waves - Acquisition and Processing - Kiel Bay 77

6.1 Introduction . . . . . . . . . . . . . . . . . . 78

6.2 Seismic experiments . . . . . . . . . . . . . . 78

6.2.1 Time synchronization of the source and receiver systems . . . . . . 82

6.2.2 Determining and achieving the required shot interval . . . . . . . 83

6.3 Data examples, acquisition and preprocessing . . . . . . . . . . 83

6.3.1 Comparison of different receiver systems . . . . . . . . . . . . . 84

6.3.2 Scholte wave dispersion at different geological sites . . . . . . . . 91

6.4 Conclusions . . . . . . . . . . . . . . . . . . . . . . 94

7 Acoustic Guided Waves - Marine Permafrost in the Laptev Sea, Siberia 99

7.1 Introduction . . . . . . . . . . . . . . . . . . . . . 100

7.2 Multichannel Seismic Data . . . . . . . . . . . . . . . . . . 101

7.2 .1 Study Area . . . . . . . . . . . . . . . . . . . . 101

7.2.2 Seismic Lines and Acquisition Parameters . . . . . . . . . . . . 103

7.2 .3 Data Example . . . . . . . . . . . . . . . . . 103

7.2.4 Regional Variation of Dispersion Curves . . . . . . . . . . . . . . . 105

7.3 Sensitivity of Seismic Parameters of Dispersive Guided Waves . . . . . . . . 110

7.3.1 Reference Model . . . . . . . . . . . . . . . . 111

7.3.2 Sensitivity of the Acoustic Guided Modes to Variations of Sediment

Properties ........................ 111

7.4 Inversion . . . . . . . . . . . . . . . . . . . . 118

7.4.1 Inferring Seismic Parameters from Shot Gathers in two Stages . . . . 118

7.4 .2 Inversion Results . . . . . . . . . . . . . . . . . . . . 121 
7.4.3 Resolution Analysis . . . . . . . . . . . . . . . . . . 122

7.5 Conclusions . . . . . . . . . . . . . . . . . . . . . 124

8 Dispersive Wave Studies in the Arkona Basin Natural Laboratory, Baltic Sea 127

8.1 Introduction . . . . . . . . . . . . . . . . . 128

8.2 The Arkona Basin Study Area . . . . . . . . . . . . . . . . . . . 128

8.3 A Towed Data Acquisition System . . . . . . . . . . . . . . . . . . . . . . . . . . . . . . . . . . . . . . . .

8.4 Acquisition Tests . . . . . . . . . . . . . . . . . . . . . . . . . . . . . . . . . . . . . . . . .

8.4 .1 Towing Depth . . . . . . . . . . . . . . 132

8.4 .2 Offset Range . . . . . . . . . . . . . . . . . . . 135

8.4.3 Data Sets . . . . . . . . . . . . . . . . . 135

8.5 Determining Sediment Stratigraphy and Velocity Models . . . . . . . . . . . . 138

8.6 Acquisition and Preprocessing of the OBS-Data . . . . . . . . . . . . . . . 144

8.7 Analysis of Dispersive Wavefields . . . . . . . . . . . . . . . . . 147

8.8 Inferring the Shear Wave Velocity by Inversion of the Wavefield Spectra . . 152

8.9 Conclusions . . . . . . . . . . . . . . . . . . 158

\section{Discussion of Results}

9 Results, Discussion and Conclusions $\quad 161$

9.1 Acquisition of dispersive waves . . . . . . . . . . . . . . . . . 161

9.2 Inversion of dispersive waves . . . . . . . . . . . . . . . . . . . 164

9.3 Conclusions . . . . . . . . . . . . . . . . . . . 166

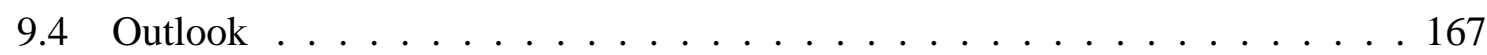

$\begin{array}{ll}\text { References } & 169\end{array}$

$\begin{array}{ll}\text { List of Figures } & 181\end{array}$

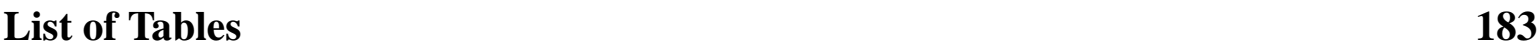

A Methods $\quad \mathbf{1 8 5}$

A.1 Acquisition Restrictions of the Stationary-Receiver Experiment . . . . . . . 185

A.2 Influence of Acquisition Geometry . . . . . . . . . . . . . . . 186

B Data Acquisition $\quad 189$

B.1 Research Cruises . . . . . . . . . . . . . . . . . . . . . . . . 189

B.1.1 List of research cruises . . . . . . . . . . . . . . . . . 189

B.1.2 List of stations and profiles of the cruises Po266, Al188 and Al201 . . 189 
B.1.3 Corrections for Sound Speed in Water . . . . . . . . . . . . . . . 190

B.1.4 Data conversion and preparation . . . . . . . . . . . . . . 190

B.1.5 Navigation . . . . . . . . . . . . . . . . . . . . 191

B.2 Deep-tow streamer data . . . . . . . . . . . . . . . . . 192

B.2.1 Deep-tow acquisition tests . . . . . . . . . . . . . . . 192

B.2.2 Single channel sections of deep-tow seismic lines . . . . . . . . 193

B.2.3 Selected shot-gathers of the deep-tow streamer lines . . . . . . . . 193

C Sensitivity of Inversion Parametes 197

C.1 Sesnsitivity of Acoutic Guided Wave Dispersion in Marine Permafrost Con-

ditions . . . . . . . . . . . . . . . . . . 197

D Used Tools and Software $\quad \mathbf{2 0 5}$

D.1 Forward Algorithm . . . . . . . . . . . . . . . . . . . . . 205

D.1.1 Propagator Matrix Algorithm MSEIS and MSEISFK . . . . . . . . . . . 205

D.1.2 Mode Summation Algorithm FlsPHER . . . . . . . . . . . . . . 205

D.2 Seismic Processing . . . . . . . . . . . . . . . . . . . 205

D.2.1 Standared Processing . . . . . . . . . . . . . . 205

D.2.2 Frequency Domain Slant-stacking Algorithm . . . . . . . . . . 206

D.2.3 Dispersion Curve Inversion and Visulization . . . . . . . . . . 207

D.2.4 Inversion of full Wavefield Spectra . . . . . . . . . . . . . . . . . . . . . . . . . . . . . . . .

D.2.5 Other Tools . . . . . . . . . . . . . . . . . . 207

D.3 List of Abbrevations and Symbols . . . . . . . . . . . . . . . . 208

$\begin{array}{lr}\text { Acknowlegdements } & 209\end{array}$

$\begin{array}{ll}\text { Lebenslauf } & 210\end{array}$

$\begin{array}{ll}\text { Erklärung } & 211\end{array}$ 


\section{Chapter 1}

\section{Introduction}

Modern technology is developing at increasing pace, and we continue to use the marine environments to build structures in the coastal shelf seas, in order to bridge the transportation and information routes spanning the globe. Telephone, internet and power cables are laid from continent to continent crossing the deep seas and mid ocean ridges as well as the shelf seas. Drilling and oil production platforms are built at water depth exceeding several hundreds of meters today, not only in the shelf seas, but pushing out to the continental slopes. The need for conventional energy sources will probably require to use exploration targets in the deep sea environments in the future. Pipelines are built and maintained in the marine environment, hence surveying of cable routes as well as monitoring the burial of pipelines and cables is an ongoing task to be fulfilled covering larger distances as development progresses. Wind parks are erected in shallow marine environments using alternative energy sources. Transport routes are linked by bridges or tunnels, just like the crossing of the Great Belt in the Baltic Sea between the islands of Langeland and Fyn which require enormous static loads to be applied at the sea floor to support the structures. All these developments require to study the sediment structures and their resistance to statical and dynamical forces, prior to the construction. Hence, a method that helps to determine the strength of marine sediments in a fast and cost effective manner covering large distances and areas would be a contribution to modern offshore technology.

The strength of marine sediments, which is related to the propagation velocity of shear waves, the key parameter of investigation within this thesis, is also required in hazard analysis and slope stability in the marine environment. One can hardly imagine the devastating forces of tsunamis caused by sub marine slides from the largest known slides, e. g. the Storrega Slide in the Norwegian Sea, since they occured prior to human records. To investigate the causes of such hazards is one of the driving forces in gashydrate studies. The potential of submarine gashydrates to rapidly disolve and change the strength of marine sediments is recognised to be an important factor in marine slope stability. Furthermore, the energy po- 
tential and relevance for climate are subjects of the current investigations of gashydrates. The large amounts of methane gas bound in gashydrates at volume ratio of 1:144 (at atmospheric pressure) yields the high potential as energy source as well as the relevance to climatic processes and contribution to the methane cycle. The identification of free gas or gas-hydrate bearing sediments could not at last be a potential application, if we could develop a method to derive the sediment stability from marine seismic measurements with high area coverage.

The marine seismic methods are used to determine the dominant layers and discontinuities of marine sediments in order to infer the geological setting, as well as to determine sediment properties. Since in water only compressional waves (sound) can be excited, therefore conventionally only these are investigated. In marine sediments, as in any solid material, seismic wave energy can propagate either as compressional waves ${ }^{1}$ or shear waves ${ }^{2}$. While the latter is dominantly influenced by the grain matrix, the former is also dependent on the sediment pores, i.e. the pore fluid and saturation are dominant factors. The stability of such a porous medium is influenced by the structure of the grain matrix and therefore closely related to the shear wave velocity of the sediment. This correlation of shear strength and the shear modulus determining the shear wave velocity has been empirically established (Ayres \& Theilen, 1999). As much as these sediment properties determine the strength and behaviour with respect to the application of static loads, they also determine the response to dilatational and torsional disturbances of pressure and stresses excited and observed in seismic investigations. Therefore the excitation of seismic pulses (usually generated by imploding air cavities in water) and the monitoring of the pressure response is used to investigate the sub-surface structure of marine sediments. The methods are well developed and continously improved. For example the use and feasibility of deep-towed multi-channel seismic experiments to provide structural information on gas hydrate sediments at greatly increased resolution was recently demonstrated (Gettrust et al., 1999).

The velocity depth profile of the seismic velocities yield more information on the layers identified in the structure, but only the compressional wave velocity is directly measurable in the water column. Layer interfaces with significant contrast in seismic velocities and/or density cause reflection, refraction and also energy conversion between the different types of seismic waves. The investigation of the variation of seismic amplitudes with angle of incident of the seismic waves has been successfully applied to derive reservoir characteristics and sediment properties, sometimes also the shear wave velocity in consolidated sediments and rocks (Carcione \& Tinivella, 2000; Whitcombe et al., 2002). While it can help to determine the den-

\footnotetext{
${ }^{1}$ Compressional waves, also called P(rimary)-waves due to the fact that they arrive first in a seismic record, are characterised by particle movement in direction of wave propagation.

${ }^{2}$ Shear waves are also called $\mathrm{S}$ (secondary)-waves since their velocity of propagation is slower than the $\mathrm{p}$ wave velocity, hence they arrive second. The particle motion is perpendicular to the direction of propagation. We generally consider two components, one horizontally the other vertically polarised with respect to the coordinate system used.
} 
sity and impedance of sediments, the sensitivity of the reflection coefficients on shear wave velocity is weak for shallow marine sediments (Riedel \& Theilen, 2001). Methods to directly measure the shear wave velocity at the sea floor have been tested successfully (Gimpel, 1987; Davis, 1996). Despite of the successfull in-situ measurements of shear wave velocities, the required operational effort and the slow progress limit the usefulness. The energy conversion from compressional waves to shear waves has been used and the analysis of converted waves was successfully applied in exploration seismics to investigate reservoir characterisitcs. However, in the shallow marine environment these methods are limited in case of soft sediments with low shear wave velocites. Limitations arise from the small conversion coefficients and thus little energy available to be analysed. Additionally the convetional methods to separate and identify converted waves fail for soft marine sediments due to the near vertical angle of incident of converted waves in such conditions (Bussat, 2001).

Hence, to measure the shear wave velocity in unconsolidated marine sediments in the shallow environment, we still lack of a method easily and commonly applicable with standard marine surveys. In recent years the analysis of marine interface waves was futher developed to derive shear wave velocity information (Essen 1980, Schirmer 1980, Rauch 1986, Gimpel, 1987, Stoll et.al 1991, Ewing et.al. 1992, Krone 1997). In certain conditions the seismic energy is propagated by interface waves within the vicinity and along layer interfaces next to the propagation of body waves which was first noticed by Lord Rayleigh (1886). Lamb (1904) extended his results for a general solution for a point source, and Bromwich (1898), Scholte (1924) and Stoneley (1926) investigated the interface waves in fluids before Stoneley (1928) finaly presented a description for the solid-solid interface. These waves are merely a superposition of body waves, e.g. Rayleigh, Scholte and Stoneley waves are combined from compressional and vertically polarised shear waves (P-SV-waves). Relating to the propagation along the vacuum/solid, fluid/solid and solid/solid interfaces Rauch (1986) has classified them as Rayleigh, Scholte and Stoneley waves respectively. In case of sediment stratification, i.e. the variation of seismic properties with depth, these interface waves do exhibit dispersive characteristics. Thus their propagation velocity is frequency dependent, and the dispersion characteristic is dominantly influenced by the shear wave velocity.

On land the dispersion of surface waves has been investigated by seismologists for many years (Press \& Ewing, 1952; Nolet, 1977), but the development of a simple active experiment and analysis method, denoted as Spectral Analysis of Surface Waves (SASW) method, had large impact in the 1980s (Nazarian, 1984). McMechan and Yedlin (1981) showed that the dispersion of multi-channel seismic data could be obtained from a two-dimensional wavefield transform, which significantly enhances the resolution.

The application of this method in marine environments was tested by Stokoe et al. and Luke \& Stokoe.(Stokoe et al., 1991; Luke \& Stokoe, 1998) and investigations of the application of multi-channel analysis has started recently (Bohlen et al., 2003; Klein et al., 2000; 
Park et al., 2000; Muyzert, 2000; Bohlen et al., 1999; Xia et al., 1999; Park et al., 1999). While most authors use the enhanced seismic data to better resolve the disperison curve for inversion methods I will investigate the potential of including information from the amplitudes of the wavefield spectra.

Hence, the objective of this theses is to investigate the potential of methods to derive the shear wave velocity in shallow marine environments using marine seismic experiments and dispersion analysis methods. This may help to determine properties of marine sediments, especially the strength to support structural applications and the properties related to propagation of seismic energy. The method proposed is based on the analysis of dispersive seismic wave energy. 


\section{Part I}

\section{Theory and Methods}





\section{Chapter 2}

\section{Dispersive Waves in Marine Sediments}

\section{Contents}

2.1 Marine Sediments $\ldots \ldots \ldots \ldots \ldots$

2.2 Seismic wave propagation . . . . . . . . . . . . . 20

2.3 Poroelastic and viscoelastic material properties . . . . . . . . 23

2.4 Lamb's problem . . . . . . . . . . . . . . . . . 24

2.5 Scholte waves . . . . . . . . . . . . . . . . 26

2.6 Dispersive waves in marine conditions . . . . . . . . . . . . 28

2.6.1 Dispersive waves in a stratified medium . . . . . . . . . . . 28

2.6.2 Wave guides and leaky modes . . . . . . . . . . . . 30

2.6.3 Dissipative and other dispersion effects . . . . . . . . . . 33

Before I start with the introduction of dispersive seismic waves I will briefly discuss the key properties of marine sediments which are the medium of propagation of the dispersive waves discussed in this thesis. The intention is to recall the simplifications of the concepts used to describe the propagation of seismic wavefields in terms of homogeneous layered media. 


\subsection{Marine Sediments}

Sedimentation processes are the key to the building of marine sediments. The materials that comprise new sediments vary in size, shape and constituents, but the common feature is the formation of the sediments by deposition of particles through water at hydrostatic pressure. The increase of overburden pressure with depth is far less profound in the marine environment than on land and therefore we find a large variety between loosely and dense compacted sediments. In places where ocean currents, tides or wave action hinder sedimentation or cause erosion, we find hard rock near or at the sea floor. The range from soft to hard sub surfaces in the marine environment is therefore much greater than observed on land.

To generalize a description of properties of these highly variable materials we consider well established theories on porous media as an appropriate description of properties of marine sediments. For details of these theories and their application I refer to the monographs by Bourbie et.al, Dullian and Schön or a comprehensive summary of the theoretical and practical implication of this concept with respect to seismic analysis published by Mavko et.al. (Bourbie et al., 1987; Dullien, 1992; Schön, 1998; Mavko et al., 1998). In this section some key properties of porous media are summarized with respect to the importance for seismic wave propagation.

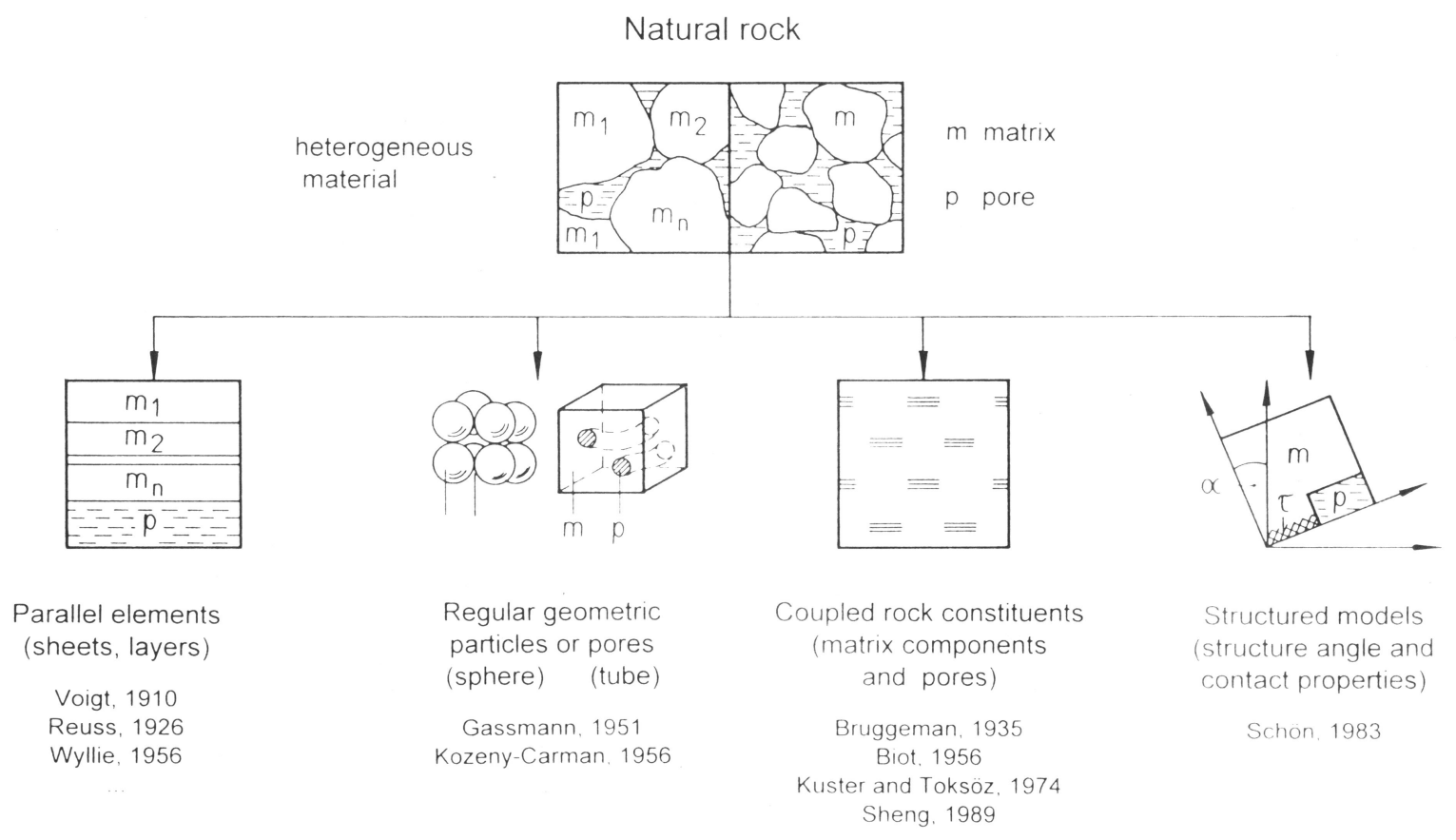

Figure 2.1: Models and theories to describe porous media (Schön, 1998) 
Marine sediments in general consist of some matrix material composed of grains of different material and size, some space between the individual grains (pores) which is usually filled with some pore fluid, sea water in most cases, but other liquids or gas, occasionally, too (Fig. 2.1). The interaction of grains, grain contact areas, pore fluid as well as the ambient physical environment determine the physical and geotechnical properties of the marine sediment. These in terms determine its properties and the behaviour with respect to the propagation of elastic or viscoelastic disturbances. Hence, we can expect, that measurements and derivations of seismic parameters can be used to determine information on sub surface structure and composition. However, the physical and chemical processes involved in the bounding of a mixture of individual materials as well as their reaction to stress induced dilatational and torsional response to seismic shock waves is rather complex and the number of theories developed to account for the various processes is large. Schön (1998) groups the numerous publications and theories according to their approach as presented in figure 2.1.

These theories present a comprehensive and fundamental approach to describe the interaction between the constituents of a composite material with respect to stress induced energy propagation, we require a large number of parameters to fully describe the properties of the sediment. The grain-to-grain contact models based on the concept of Hertz, 1881, involve parameters of the constituents (grain radius, contact areas, packing) to determine the density of the sediment from its constituents. The physical interaction between the grains is described to predict the response to external forces. Other models include the effect of saturation of the pores with fluids or gas and involve additional fluid parameters (e.g. fluid saturation and viscosity of the fluid). Biot's theory (Biot, 1956, 1962) and the further development e.g. by Dvorkin and Nur (1993) include other fluid flow processes to just mention a few of the numerous theories.

In order to reduce the number of free (unkown) parameters required to describe a sediment, further assumptions and limitations have to be made. Secondly, most physical processes determining these properties act on a scale much smaller than the wave length of the seismic wavefield inducing the stress or strain. Hence, while the sediment may be of a complex structure up to the $\mathrm{cm}$ scale, it may act virtually like an homogeneous sediment for seismic energy and frequencies corresponding to much larger wavelength. Thus we seek to describe a marine sediment appropriately by a simplified model on the scale we are able to measure with seismic experiments.

We therefore follow the principle idea of the theories to derive the complex structure of a porous composite material in terms of its structure and constituents in order to obtain material properties of the medium which can be compared to those of a homogeneous effective medium on the scale relevant for the seismic experiments to be performed for their investigation. This principle enables to separate the task of deriving the effective material properties from seismic experiments from the sediment physical description of the medium. In a sec- 
ond stage the seismic properties of the effective medium can be used to derive structural information from the sediment e.g. using additional information on the constituents.

Using this concept of composite materials to derive properties of an effective medium enables us to separate the task to interpret the measured seismic velocities as variations in the distribution and properties of the constituents of the material of observation. We first measure the properties of the effective medium using the well established methods applicable to homogeneous media, and then interpret these effective properties in terms of the appropriate sediment physical model.

\subsection{Seismic wave propagation}

In order to utilize the measurements of seismic wave energy to derive sediment properties we have to understand the principles of excitation and propagation of seismic energy, which lead to the description of the relevant dispersive seismic interface waves. Using the principles of continuum mechanics, the medium is described as a continuum of particles. Forces acting between the particles induced by their displacement of the equilibrium steady state are balanced by the volumetric distribution of stresses $\sigma$ with the volume density of momentum forces $\rho \ddot{\vec{u}}$ and locally applied forces $\overrightarrow{f_{V}}$. This leads to the equation of motion (2.1) which yields the vector displacement $\vec{u}$, the stress tensor $\sigma$ and the partial differentials in time and space $^{1}$.

$$
\rho \ddot{\vec{u}}-\operatorname{Div} \sigma=\overrightarrow{f_{V}}
$$

\section{Linear stress-strain relation - Hooke's law}

In order to solve the above system of coupled differential equations, we seek to transform this problem into a system of ordinary differential equations, which will eventually lead to the elastic wave equation. The first step is to assume a linear relation between stress and strain (Hooke's law). The vector displacement $\vec{u}$, which is the observable in seismic measurements, is related to the symmetric strain tensor $\epsilon$ which denotes the dilatational and torsional deviations producing internal stresses within the body governed by the stress-strain relation (2.2). In this equation the 9 component strain tensor is comprised of the three dilatational strains expressed by $\epsilon_{i i}=\frac{\partial u_{i}}{\partial x_{i}}, \forall i=x, y, z ; \sum x_{i}=(x, y, z)$ and the three shear components of strain $\epsilon_{i j}=\epsilon_{j i} \forall i, j=x, y, z$. The 9 component stress tensor is denoted respectively.

$$
\begin{gathered}
\sigma_{i j}=M_{i j k l} \varepsilon_{k l} \\
\varepsilon_{i j}=\frac{1}{2}\left(\frac{\partial u_{i}}{\partial x_{j}}+\frac{\partial u_{j}}{\partial x_{i}}\right)
\end{gathered}
$$

\footnotetext{
${ }^{1} \ddot{u}=\frac{\delta^{2} u}{\delta t^{2}}$ denotes the second derivative of the displacement with respect of time, and Div $\sigma$ denotes the divergence of the stress field.
} 
The mass density $\rho$ and the relation between stress tensor $\sigma$ and strain tensor $\varepsilon$ given by the 4th order tensor of elastic constants $M$ are the material properties which in general vary with time and space. The tensor $M$ has 81 elements from which are at most 21 independent due to its symmetry. Hence, we require 21 elastic material constants plus the mass density distribution to describe the motion of particles in an arbitrary elastic medium. While this may be required in elastic solids of complex mineral structures with large anisotropy, we consider it appropriate to assume that marine sediments can be expressed in terms of homogeneous, isotropic effective media. Then material symmetry further reduces the number of independent elements, and we remain with two independent elements, if we consider it appropriate to assume that the stress-strain relation is independent of any direction characteristic for an isotropic, homogeneous medium. Generally the sum of the three dilatational strains, also denoted as the cubic dilatation $\theta=\sum \varepsilon_{i i}$, is invariant of the choice of coordinate system, but using the system of the principle axes of strain, the shear components of strain vanish and all strain components are given by $\theta$.

Then $\mathrm{M}$ reduces to the two independent elements $\lambda, \mu$ referred to as Lamé constants, and the stress-strain relation reduces to equation 2.3.

$$
\sigma_{i j}=\lambda \theta \delta_{i j}+2 \mu \varepsilon_{i j}
$$

While $\lambda$ lacks any physical meaning, the second constant $\mu$ corresponds to the shear modulus or rigidity, which determines the stress-strain relation for tangential stress (Fig. 2.2a). The former can be related to the compressibility $\beta$ of the medium denoted by its
a) pure shear stress $\left(\sigma_{k l}\right)$
b) uniform compressional stress $\left(\sigma_{k k}\right)$

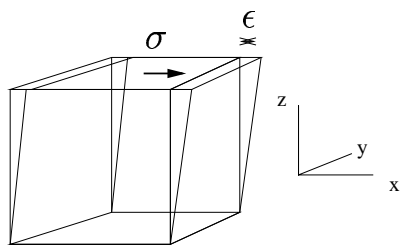

shear modul (rigidity) $\mu$

$$
\begin{gathered}
\sigma_{k l}=2 \mu \varepsilon_{k l}, k \neq l \\
\text { with } \varepsilon_{k k}=0
\end{gathered}
$$

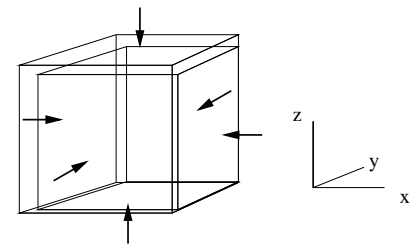

bulk modul $\kappa$

$$
\begin{gathered}
\Sigma_{k} \sigma_{k k}=3 \kappa \Sigma_{k} \varepsilon_{k k} \\
\text { with } \varepsilon_{k l}=0, \quad k \neq l
\end{gathered}
$$

Figure 2.2: The stress-strain relation for an unconfined body. The supscripts $k, l$ represent the components $x, y, z$.

recipicle in terms of the bulk modulus $\kappa=1 / \beta$ and the rigidity $\mu$ by $\lambda=\kappa-\frac{2}{3} \mu$. The bulk modulus describes the stress-strain relation for an unconfined body subject to equal compression from all directions (Fig. 2.2b). 
a) uni-axial compression $\left(\sigma_{11}\right)$

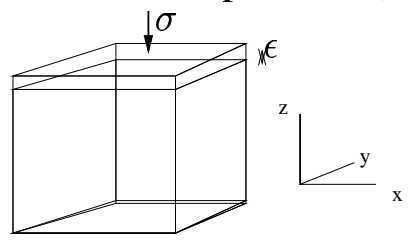

compressional wave modul $K=\kappa+\frac{4}{3} \mu$

$$
\begin{gathered}
\sigma_{33}=K \varepsilon_{33}, \\
\text { with } \sigma_{11,22,12,13,23}=0
\end{gathered}
$$

b) dilatational contraction $\left(-\varepsilon_{11}\right)$

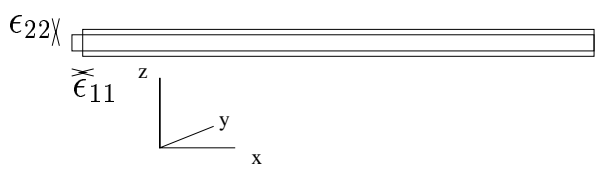

Young's modul $E$

$$
\begin{gathered}
\nu=-\varepsilon_{22} / \varepsilon_{11} \text { and } \sigma_{11}=E \varepsilon_{11} \\
\text { with } \sigma_{22,33,12,13,23}=0
\end{gathered}
$$

Figure 2.3: Stress-strain relation for a confined body. The subscripts 1,2,3 denote the components of principle stresses, which conicide with the components $x, y, z$ in the coordinate system specified.

Next to the rigidity and bulk modulus, the Poisson ratio $\nu=-\varepsilon_{22} / \varepsilon_{11}$ relating the tangential extension and dilatational contraction due to uni-axial compression ( $\sigma=E \varepsilon, \mathrm{E}=$ Young's module ) is often used to characterize the elastic properties of a medium. In the situation relevant to seismic wave propagation we find a confined body subject to compressional stresses with the corresponding module $K=\kappa+\frac{4}{3} \mu=\lambda+2 \mu$.

Hence, under the assumption that the sub surface material properties are constant we require only three material constants, e.g. the moduli $\kappa$ and $\mu$ as well as the density $\rho$, to fully determine the elastic behavior of stress and strain. Therefore by knowing these material constants and the distribution of dynamic stress and strain within the medium at one instance in time (initial value conditions), as well as at the boundary of the system (boundary conditions), we can predict the propagation of stress and strain within the medium at all times (Tab. 2.1).

\begin{tabular}{|c|c|c|}
\hline initial value condition & stress $\sigma(t, x, y, z)$ & $\sigma_{0}(x, y, z)$ at $t=0$ \\
& strain $\varepsilon(t, x, y, z)$ & $\varepsilon_{0}(x, y, z)$ at $t=0$ \\
\hline \hline boundary condition & dilatation $u$ & $u$ continous \\
& stress $\sigma$ & $\sigma$ known at all boundaries \\
\hline
\end{tabular}

Table 2.1: Initial and boundary conditions

The number of material properties increases with complexity of the mineral structure of the material, since then the propagation of stress will differ for directions in line with the mineral alignment or at arbitrary angles to it. 


\subsection{Poroelastic and viscoelastic material properties}

In order to introduce absorption of seismic wave energy we extend the former derivations for elastic media to include viscous damping of the elastic deformation. The principle and formal introduction is obtained by generalizing the material properties of the medium to be causally dependent on the evolution of the material property. This memory effect of the material is formulated for the stress-strain relation

$$
\begin{gathered}
\sigma_{i j}(t)=\int_{-\infty}^{\infty} M_{i j k l}(\tau) \epsilon(t-\tau) d \tau \\
M(\tau)=0 \quad \text { at } \quad \tau<0
\end{gathered}
$$

The integral in eq. 2.4 corresponds to a multiplication of the complex Fourier coefficients

$$
\tilde{\sigma}_{i j}(\omega)=\tilde{M}_{i j k l}(\omega) \tilde{\epsilon}_{k l}(\omega)
$$

and therefore is easily adapted in the solution of the wave equation as described previously. We obtain complex moduli and complex seismic velocities.Plastically, the complex stress-strain relation is equivalent to introducing an additional attenuating mechanism next to the spring, which was the only rheological elementof the linear elastic solid discussed so far. This additional attenuating element is the dashpot with viscous damping of the movement, hence the temporal change of the displacement $\dot{\epsilon}$.

\begin{tabular}{|c|c|}
\hline spring & $\sigma=D \epsilon$ \\
\hline \hline dashpot & $\sigma=\eta \dot{\epsilon}$ \\
\hline
\end{tabular}

The material properties are therefore extended by the viscous element $\eta$, with contribution to the imaginary part of the complex tensor $\tilde{M}$, which will lead to a dissipative term in the wave equation. The spring constant $\mathrm{D}$ contributes to the real part of the complex tensor $\tilde{M}$, hence is identical with the elastic $M$ in the absence of viscous damping. The attenuation in form of the dissipative contribution of the complex moduli can be quantified in form of the definition of the dimensionless property $Q=\frac{\Re(\tilde{M})}{\Im(\tilde{M})}$.

Generally a combination of these rheological elements is used to describe the viscoelastic material. The standard linear solid is the most simple representation of a solid material (Zener, 1948). It consists of two springs and a dashpot as shown in fig. 2.4. The attenuation as well as the velocity of propagation in such a medium is dependent on frequency and on the combination of the rheological constants of the material. Two simplifications of the standard linear solid, the Maxwell body and the Kelvin-Voigts body (2.4), enable to derive asymptotical limits for the static $\left(M_{\omega=0}\right)$ and high frequency $\left(M_{\infty}\right)$ case. 

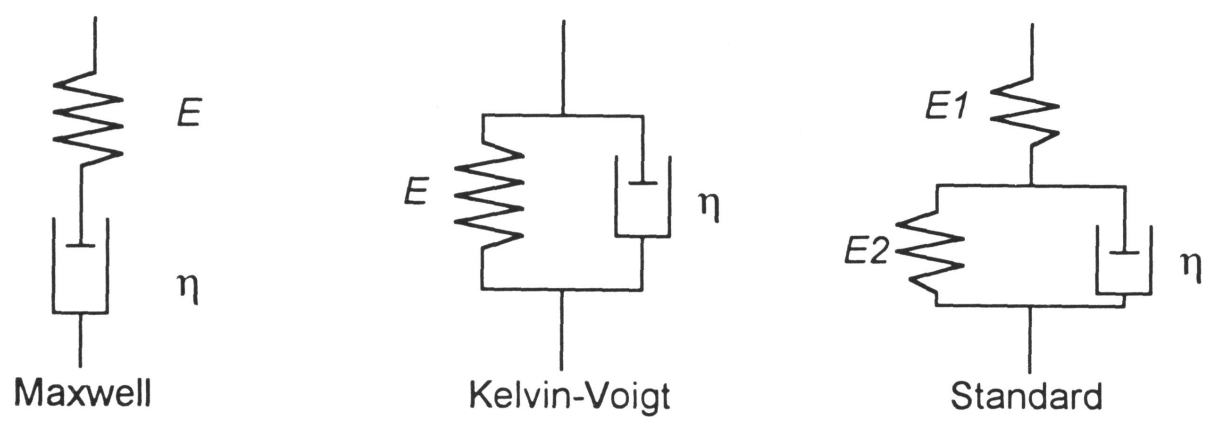

Figure 2.4: Rheological bodies to describe a viscoelastic material (in Mavko, 1998)

\subsection{Lamb's problem}

The solution of the wave equation for a vertical force acting on a homogeneous halfspace was published by Lamb (Lamb, 1904) and therefore called Lamb's problem. Referring to Lamé's theorem, the displacement vector $\vec{u}$ from the equation of motion (2.1) is separated into rotational-free and divergence-free components, which can be described by Helmholtz potentials. These are two independent seismic potentials, the scalar potential $\Phi$ and the vector potential $\vec{\Psi}$, which lead to separate wave equations (2.5)

$$
\begin{aligned}
\nabla^{2} \Phi & =\frac{1}{\alpha^{2}} \frac{\partial^{2} \Phi}{\partial t^{2}} \\
\nabla^{2} \vec{\Psi} & =\frac{1}{\beta^{2}} \frac{\partial^{2} \vec{\Psi}}{\partial t^{2}}
\end{aligned}
$$

where $\Phi$ and $\vec{\Psi}$ denote the compressional and shear potentials respectively. We obtain solutions for two types of body waves, the compressional (P) and shear (S) waves and can identify that $\alpha$ and $\beta$ correspond to their velocities of propagation respectively (Aki $\&$ Richards, 2002).

$$
\alpha=\sqrt{\frac{\lambda+2 \mu}{\rho}} \quad \beta=\sqrt{\frac{\mu}{\rho}}
$$

Here, $\lambda$ and $\mu$ correspond to the Lamé constants described in section 2.2 and $\rho$ denotes the material density. The particle motion as described by the displacement vector $\vec{u}=(u, v, w)$ can be determined from the potentials and is depicted in fig. 2.5. In assumption that the displacement is independent of the y direction we obtain for the components $u, v, w$

$$
u=\frac{\partial \Phi}{\partial x}-\frac{\partial \Psi_{2}}{\partial z}, \quad v=\frac{\partial \Psi_{1}}{\partial z}-\frac{\partial \Psi_{3}}{\partial x}, \quad w=\frac{\partial \Phi}{\partial z}+\frac{\partial \Psi_{2}}{\partial x} .
$$

Due to the nature of the directional force in the case of a vertical hammer blow at the surface, the particle movement of the shear wave is purely in the vertical plane, hence only 


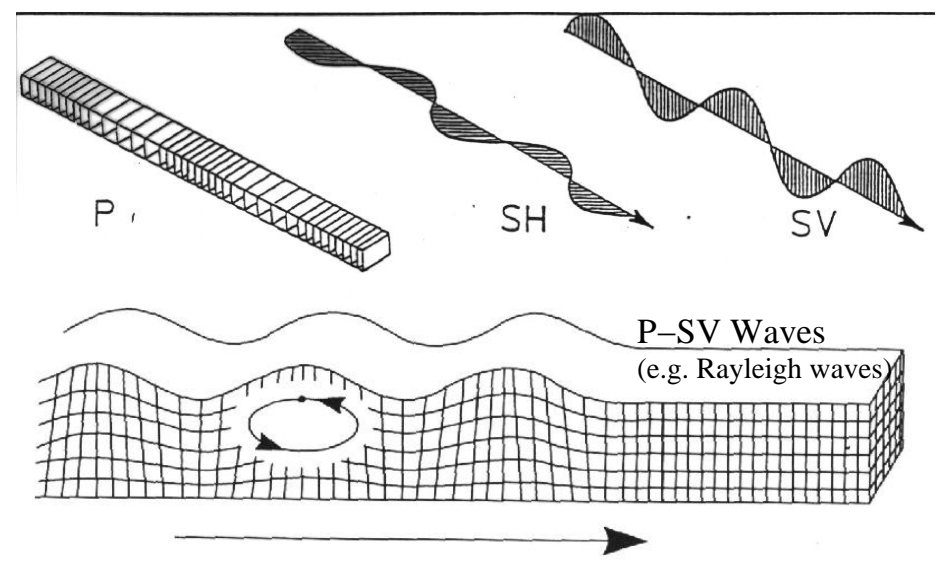

Figure 2.5: Particle movement for $\mathrm{P}, \mathrm{SV}$ and $\mathrm{SH}$ body waves (top) and the P-SV interface wave (bottom)

vertically polarized shear waves (SV) exist in this case. An explosive source at the surface acting in all directions also excites shear waves with particle motion deviating from the vertical plane. The propagation of horizontally polarized shear waves $(\mathrm{SH})$ propagating perpendicular to the vertical plane and the SV-waves are expressed by independent seismic potentials and may have independent seismic velocities. Since in practice, horizontally polarized shear waves can not be excited by seismic sources in the water column, these types are not of interest in the following, but just mentioned for completeness.

Lord Rayleigh showed as early as 1885 , that an interface wave propagates at the free surface boundary to the solid medium, which is therefore called Rayleigh wave. The amplitude of particle motion of this wave decays away from the interface and vanishes for infinite depth. The energy propagates in direction of the interface plane. While the two body wave solutions are homogeneous waves, the Rayleigh wave is composed of inhomogeneous components of $\mathrm{P}$ and SV body waves, and the energy is bound to the interface, decaying with increasing distance. Hence, the boundary conditions of continuous stress and strain at interface $(\mathrm{z}=0)$ and amplitudes vanishing at infinity must be fulfilled by the solution, which we seek by the following ansatz of plane waves with wave number $k$ propagating in the $x$ direction.

$$
\Phi=A \cdot e^{k \zeta z} e^{i(\omega t-k x)} \quad \text { and } \quad \Psi=B \cdot e^{k \xi z} e^{i(\omega t-k x)}
$$

where

$$
\zeta^{2}=\left(1-\frac{c^{2}}{\alpha^{2}}\right) \quad, \quad \xi^{2}=\left(1-\frac{c^{2}}{\beta^{2}}\right), c=\frac{\omega}{k}
$$

In order to fulfill the requirement of exponential decay it is obvious that $\zeta$ and $\xi$ must be real and therefore the velocity of propagation $c$ must be smaller than both body wave veloci- 
ties $\alpha$ and $\beta$. With the boundary conditions at $\mathrm{z}=0$ we obtain a linear system of equations with non-trivial solutions if the determinant of the matrix system vanishes, which I will discuss in more detail for the more general case of two halfspaces below. However, in this case this leads to the Rayleigh equation (2.10), which determines the velocity of propagation of the Rayleigh wave $c_{R}$ in relation to the body wave velocities $\alpha$ and $\beta$.

$$
\left(2-\frac{c_{R}^{2}}{\beta^{2}}\right)^{2}-4 \sqrt{1-\frac{c_{R}^{2}}{\alpha^{2}}} \sqrt{1-\frac{c_{R}^{2}}{\beta^{2}}}=0
$$

The Rayleigh wave has been studied intensively, since earthquake damage is mostly caused by this wave type due to its high amplitudes. The amplitudes decay exponentially with distance from the interface and the particle motion is elliptically polarized in the plane determined by the direction of propagation and the normal to the interface. These are general characteristics of interface waves which will be subject of this study. The measurement of the particle motion can therefore help to identify interface waves.

\subsection{Scholte waves}

Interface waves can exist not only at the free surface, i.e. an air-solid contact, as introduced in the previous section. Interfaces at fluid-solid and solid-solid boundaries also give rise to waves propagating along the interface similar to Rayleigh waves, but due to the difference in the boundary conditions their velocity of propagation differs, too. Scholte (1924) studied the influence of the water layer on the interface wave intensively and therefore today this wave is often referred to as Scholte wave or modified Rayleigh wave. At the solid-solid interface the corresponding interface waves are termed Stoneley waves and the boundary conditions infer continuous stress and displacement at the interface (Rauch, 1980). Denoting stress and displacement as defined in section 2.2 the boundary conditions are summarized in table 2.2.

Since in marine conditions the fluid layer is always present, I will discuss the derivation of the velocity of propagation and the particle motions in more detail. At the fluid-solid interface the tangential stress must vanish, while the vertical displacement and stress must be continuous. Therefore, we obtain the wave equation for a liquid and a solid halfspace separated at the boundary $\mathrm{z}=0$; with the ansatz in equation (2.8) for both halfspaces denoted by the index $\mathrm{m}$ ( $\mathrm{m} \exists f, s$ for fluid or solid, respectively) (d'Arnaud Gerkens, 1989).

$$
\begin{array}{ll}
\frac{\partial^{2} A_{m}(z)}{\partial z^{2}}+k^{2}\left(\frac{c_{S c h}^{2}}{\alpha_{m}^{2}}-1\right) & A_{m}(z)=0 \\
\frac{\partial^{2} B_{m}(z)}{\partial z^{2}}+k^{2}\left(\frac{c_{S c h}^{2}}{\beta_{m}^{2}}-1\right) & B_{m}(z)=0
\end{array}
$$

The general solution is a linear combination of 


\begin{tabular}{|c|c|c|}
\hline Rayleigh wave & Scholte wave & Stoneley wave \\
\hline vacuum-solid & fluid-solid & solid-solid \\
\hline $\begin{array}{l}\sigma_{x z}=0 \\
\sigma_{z z}=0\end{array}$ & $\begin{array}{c}\sigma_{x z_{l}}=\sigma_{x z_{s}}=0 \\
\sigma_{z z_{l}}=\sigma_{z z_{s}} \\
u_{z_{l}}=u_{z_{s}}\end{array}$ & $\begin{aligned} \sigma_{x z_{s 1}} & =\sigma_{x z_{s 2}} \\
\sigma_{z z_{s 1}} & =\sigma_{z z_{s 2}} \\
u_{z_{s 1}} & =u_{z_{s 2}} \\
u_{x_{s 1}} & =u_{x_{s 2}}\end{aligned}$ \\
\hline $\begin{array}{c}c_{R}=a \cdot \beta \\
0.8<a<0.98\end{array}$ & $c_{R}<c_{S c h}<\min \left(\alpha_{l}, \beta_{s}\right)$ & $\max \left\{\begin{array}{l}c_{R_{s 1}} \\
c_{R_{s 2}}\end{array}\right\}<c_{S t}<\min \left\{\begin{array}{l}\beta_{s 1} \\
\beta_{s 2}\end{array}\right\}$ \\
\hline
\end{tabular}

Table 2.2: Interface wave types as classified by Rauch (1980). The subscript $l$ denotes the fluid properties of the liquid halfspace and s the solid properties, subscripts $s 1$ and $s 2$ denote the solid upper and lower halfspaces respectively.

$$
A_{m}^{+}\left(+\zeta_{m}\right), A_{m}^{-}\left(-\zeta_{m}\right) \text { and } B_{m}^{+}\left(+\xi_{m}\right), B_{m}^{-}\left(-\xi_{m}\right)
$$

where $\zeta_{m}$ and $\xi_{m}$ are given by $\zeta_{m}^{2}=\left(1-c_{S c h}^{2} / \alpha_{m}^{2}\right)$ and $\xi_{m}^{2}=\left(1-c_{S c h}^{2} / \beta_{m}^{2}\right)$, corresponding to eq. 2.9. Again, as for the Rayleigh equation, but with boundary conditions relating to the fluid-solid contact at $\mathrm{z}=0$ we obtain the linear system of equations, which explicitly reads with indices $f, s$ denoting the fluid and solid material

$$
\begin{array}{cccccc} 
& - & 2 i \zeta_{s} & A_{s}+ & \left(\xi_{s}+1\right) & B_{s}=0 \\
\zeta_{f} & A_{f}+ & \zeta_{s} & A_{s}+ & i & B_{s}=0 \\
\lambda_{f}\left(\zeta_{f}^{2}-1\right) & A_{f}- & \left(\lambda_{s}\left(\zeta_{s}^{2}-1\right)+2 \mu_{s} \zeta_{s}^{2}\right. & A_{s}+ & 2 i \mu_{s} \xi_{s} & B_{s}=0
\end{array}
$$

where the first equation of (2.12) relates to the continuity of stress in $\mathrm{x}$ direction, and the others to the continuity of strain and stress in z direction. A non-trivial solution for the three unknowns $A_{f}, A_{s}$ and $B_{s}$ in (2.12) is found, if the determinant of this linear system vanishes which leads to the Scholte equation (2.13).

$$
\frac{c_{S c h}^{2} \rho_{f} \sqrt{1-c_{S c h}^{2} / \alpha_{s}^{2}}}{\beta^{2} \rho_{s} \sqrt{1-c_{S c h}^{2} / \alpha_{f}^{2}}}+\frac{\beta^{2}}{c_{S c h}^{2}}\left[\left(2-c_{S c h}^{2} / \beta^{2}\right)^{2}-4 \sqrt{1-c_{S c h}^{2} / \alpha_{s}^{2}} \sqrt{1-c_{S c h}^{2} / \beta^{2}}\right]=0
$$

The Scholte wave velocity $c_{S c h}$ is related to the speed of sound in the fluid phase $\alpha_{f}$ and the compressional $\alpha_{s}$ and shear $\beta$ wave velocities of the solid and the densities of the fluid $\rho_{f}$ and solid $\rho_{s}$, respectively. Note, that the term in brackets [ ] is the Rayleigh determinant from equation 2.10, hence some authors denote this wave as modified Rayleigh wave.

The particle displacement of the interface wave is obtained by inserting the Ansatz for the potentials into equation 2.7 for the displacement components. Formulating the displacement 
in cylinder coordinates, which reflects the invariance of horizontal direction of propagation from the point source, we obtain for the radial and vertical displacement

$$
\begin{aligned}
u(r, z, t) & =-i \cdot A \cdot a(z) \cdot e^{i(\omega t-k r)} \\
w(r, z, t) & =A \cdot b(z) \cdot e^{i(\omega t-k r)}
\end{aligned}
$$

where $A$ is the amplitude factor, while the real functions $a(z)$ and $b(z)$ describe the amplitude decay with depth of both components. It is obvious, that the two components $u, w$ are out of phase by $\frac{\pi}{2}$ relative, hence the particle motion is elliptically polarized. The normalized functions $a(z)$ and $b(z)$ are shown in fig. 2.6. The maximum amplitude of the radial displacement at depth $z=\lambda / 3$ indicates, that the sensitivity to velocity changes will be most significant at this depth. The vertical and radial displacement in the water column decay rapidly, but we observe that a significant amount of wave energy is propagated within the lower part of the water column. However, if the water depth is small compared to the wave length, we obtain significant amplitude levels at the water surface, yet within the derivation of the Scholte wave I have so far assumed an infinite fluid halfspace. The corresponding interface wave has a propagational velocity solely determined by the seismic velocities of the halfspace, the sound speed in water and the density contrast at the sea bed. Thus the interface waves exhibit no dispersion, since the equations 2.13 as well as 2.10 are independent of frequency. The amplitudes of the radial and vertical displacement are non zero at distances up to one wavelength away from the interface for sediment parameters commonly observed in marine sediments. The transit from an infinite towards a shallow water layer is introduced at the same time as the layering of the sediment structure and subject of the following section.

\subsection{Dispersive waves in marine conditions}

Many different physical phenomena give rise to dispersion of seismic velocities, but by far the most dominant effect observed is the dispersion of seismic interface waves due to layers of varying seismic velocities. Therefore I will continue with the description of Scholte waves for a layered sub surface and discuss the various other causes of dispersion afterwards.

\subsubsection{Dispersive waves in a stratified medium}

In the marine environment it may seem justified that sediment properties are predominantly varying with depth, since this is the direction of increase of hydrostatic pressure, and the direction in which any changes in depositional processes on geological time scales will be stored as the geological record. We therefore generalize our sediment model as a vertical stack of homogeneous layers of arbitrary thicknesses and seismic parameters, each layer representing an effective medium as discussed above. Each layer is believed to be in welded 


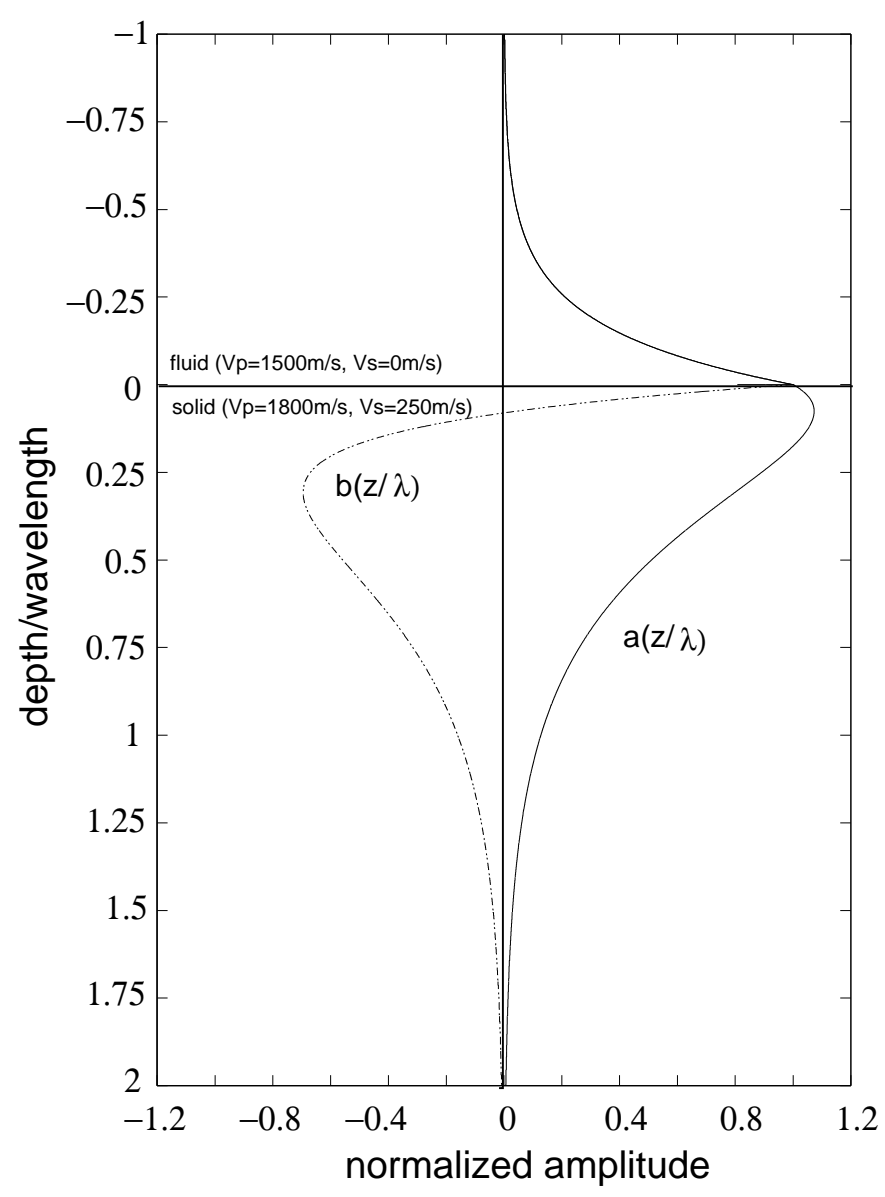

Figure 2.6: Vertical (a) and horizontal (b) component of the normalized particle displacement as a function of depth for the Scholte wave 
contact with the next and the propagation of seismic energy across the interfaces is formulated by the appropriate boundary conditions. In a layered medium either of the above interface wave types exhibits dispersion, if the seismic velocities vary with depth, since the penetration of the wave energy is dependent on frequency. In the marine environment we consider the sea bed as the liquid-solid interface which gives rise to a Scholte wave.

The relation for the non-dispersive propagation of the Scholte wave valid for two halfspaces given in equation (2.13) can be used to obtain the Scholte wave velocity at high frequency, since then the energy has already decayed at short distance away from the interface without 'noticing' the properties of adjacent layers. Assuming shallow water depth and low frequencies, in contrast, the influence of the water layer can be neglected, and the velocity of propagation approaches the Rayleigh wave velocity for the sub bottom layer properties. Hence, for a solid half space overlaid by a fluid layer we obtain an interface wave dispersion ranging from the Rayleigh wave velocity at low frequency to the Scholte wave velocity at high frequency and a transition zone in between. The characteristic frequency at which this transition occurs is related to the thickness of the water layer, since the penetration depth of interface depend on the wavelength $\lambda=1 / f$.

Introducing additional solid layers determines the dispersion relation in the transition zone. As a rule of thumb Glangeaud et.al. relate the propagation velocity at frequency $f$ to the seismic properties found at the depth corresponding to $\frac{1}{3}$ of the corresponding wavelength $\lambda$. While this is perfectly sensible for the example shown in fig. 2.6, other authors state depth relation from $1 / 2$ to $1 / 4$ wavelength (Glangeaud et al., 1999).

Generally a pure shear interface wave, the so-called Love wave with particle motion in the plane of the interface (SH-polarized), can exist in layered media, but will only be excited by shear motion at the interface. Hence it is not possible to excite Love waves within the water column and therefore is not of further interest within this study. However, the dispersion of Love and Rayleigh waves in layered media is commonly measured in land based seismological stations or seismic surveys and also successfully inverted to determine the layer velocities.

\subsubsection{Wave guides and leaky modes}

A special case of a layered medium is observed in the case of layered structure with a relatively thin layer and strong contrasts delimiting the layer. A seismic wave transmitted into the layer may be trapped inside the layer. The interference pattern of multiple reflections at the top and bottom of the layer boundary is often referred as "guided wave". The liquid layer overlaying a solid layer acts as a very good wave guide if the liquid layer is thin and the solid layer of a very rigid structure.

The geometrical approach leading to the conditions of constructive interference of multiple reverberations in the water layer is one method to derive the characteristic dispersion of 
a wave guide (Glangeaud et al., 1999). From fig. 2.7 we derive the geometric requirement

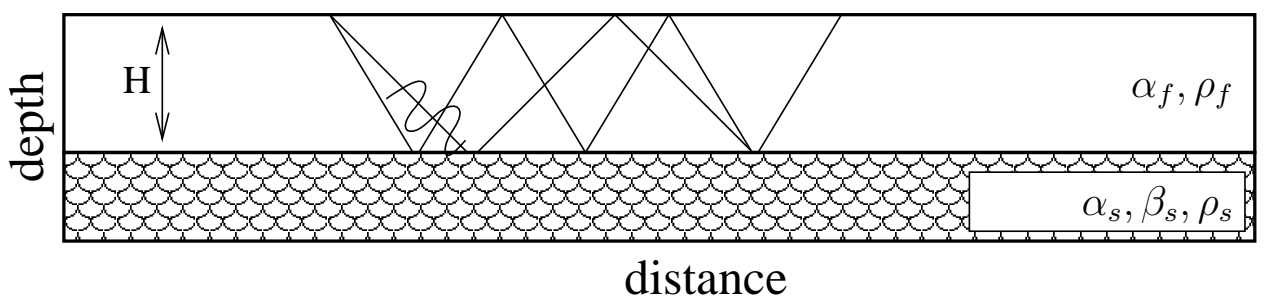

Figure 2.7: Schematic diagram for a wave guide

that the phases of two wave fronts differ by a multiple of $2 \pi$. The phase change can be determined from the water depth $H$ and the amplitude ratio $A_{f} / A_{s}=-e^{2 i \theta}$, where the phase relates by $2 \theta=2 k H \sqrt{c^{2} / \alpha_{f}^{2}-1}$. This is familiar from the ansatz used to solve (2.11), with $c$ and $k$ denoting the phase velocity and wave number of the propagating wave and $\alpha_{f}$ the compressional wave velocity in the fluid layer. Hence the phase $\theta$ is given in terms of the seismic velocities $\alpha_{f}, \alpha_{s}, \beta$ and densities $\rho_{f}, \rho_{s}$ by substituting the amplitude coefficients $A_{f}$ and $A_{s}$.

$\tan \theta=\tan \left(k H \sqrt{c^{2} / \alpha_{f}^{2}-1}\right)=\frac{\rho_{s} \beta^{4} \sqrt{c^{2} / \alpha_{f}^{2}-1}}{\rho_{f} c^{4} \sqrt{1-c^{2} / \alpha_{s}^{2}}}\left[4 \sqrt{1-c^{2} / \alpha_{s}^{2}} \sqrt{1-c^{2} / \beta^{2}}-\left(2-c^{2} / \beta\right)^{2}\right]$

We derive the period equation by adding multiples of $\pi$, and by expressing the wave number $k$ in terms of the angular frequency $\omega$ and phase velocity $c$ we obtain the dispersion relation in the form

$\tan \left(\omega H \sqrt{\frac{1}{\alpha_{f}^{2}}-\frac{1}{c^{2}}}+m \pi\right)=\frac{\rho_{s} \beta^{4} \sqrt{c^{2} / \alpha_{f}^{2}-1}}{\rho_{f} c^{4} \sqrt{1-c^{2} / \alpha_{s}^{2}}}\left[\left(4 \sqrt{1-c^{2} / \alpha_{s}^{2}} \sqrt{1-c^{2} / \beta^{2}}-\left(2-c^{2} / \beta\right)^{2}\right]\right.$

where $m \in N$ denotes the 'normal mode' number (d' Arnaud Gerkens, 1989). For the fundamental mode $(m=0)$ the relation approaches that of the Rayleigh wave in equation (2.10), if the wavelength $\lambda=\frac{2 \pi}{\omega}$ is large compared to the water depth $\mathrm{H}$, hence $c \lim _{f \rightarrow 0} c_{R}$. On the other hand, if $c$ tends to $c_{S c h}$, therefore $\alpha_{f}>c$,

$$
i \omega H \sqrt{\frac{1}{\alpha_{f}^{2}}-\frac{1}{c^{2}}}=\tan ^{-1}(i)
$$


hence $\omega H \rightarrow \infty$. Thus, for increasing layer thickness $\lim _{H \rightarrow \infty}$ as well as for $\lim _{f \rightarrow \infty}$ the equation 2.14 becomes the Scholte wave equation 2.13, which corresponds to the phenomenological derivation of the high and low frequency limits of the Scholte wave from above.

For the fundamental there is always a solution for $c<c_{\text {Rayleigh }}$ while for the modes $m>0$ normal modes only exist for frequencies above the cut-off frequency $f_{c, m}$.

\section{Leaky modes}

In the derivation of the theory of normal mode propagation the boundary condition of vanishing displacement and stresses at infinity was assumed. The energy propagation was limited to the interface and no energy was allowed to radiate into the halfspace. Since in practice energy losses into the half space are observed, the waves related to this phenomenon are called leaky modes. They can be described as inhomogeneous parts of interface waves which do not fulfill the boundary condition of normal modes at infinity. They are observed at frequencies below the cut-off frequency of normal modes. The propagation of disturbances in viscoelastic media with complex arithmetics does also include all leaky and coupled modes of the seismic wavefield. Hence, if leaky modes are observed or likely to be present in the seismic wavefields one should refrain from using (elastic) normal mode algorithms but take advantage of the wavefield propagation (or other) methods which treat the seismic wave propagation with complex arithmetics. Hence, the viscoelastic codes to compute synthetic seismic wavefields predict all wave types including leaky modes.

\section{Group and phase velocity}

Since some analyzing methods deal with the group velocity of interface wave and others with phase velocities of dispersive wave types I recall the meaning and derivation of these velocities.

Firstly, we recall that the frequency dependence of the phase velocity causes the wavelet to smear with increasing time of propagation, since the lower frequencies travel at higher speed and arrive earlier. The group velocity is defined by the frequency dependence of the phase velocity $c(\omega)$. While $c=\frac{\omega}{k}$, the group velocity $U$ is given in terms of the derivative of the frequency with respect to the wave number $U=\frac{d \omega}{d k}$, or expressed in terms of the phase velocity $\mathrm{U}$.

$$
U=c(\omega)\left[1-\frac{\omega}{c(\omega)} \frac{\delta c}{\delta \omega}\right]
$$

Therefore the group velocity describes the velocity of propagation of the wave-energy. The phase and group velocities for a solid wave guide are shown in fig. 2.8. Note the points 
of stationary group velocity, which indicate the so-called Airy phase. Contribution of this frequency range dominate the wave spectra.

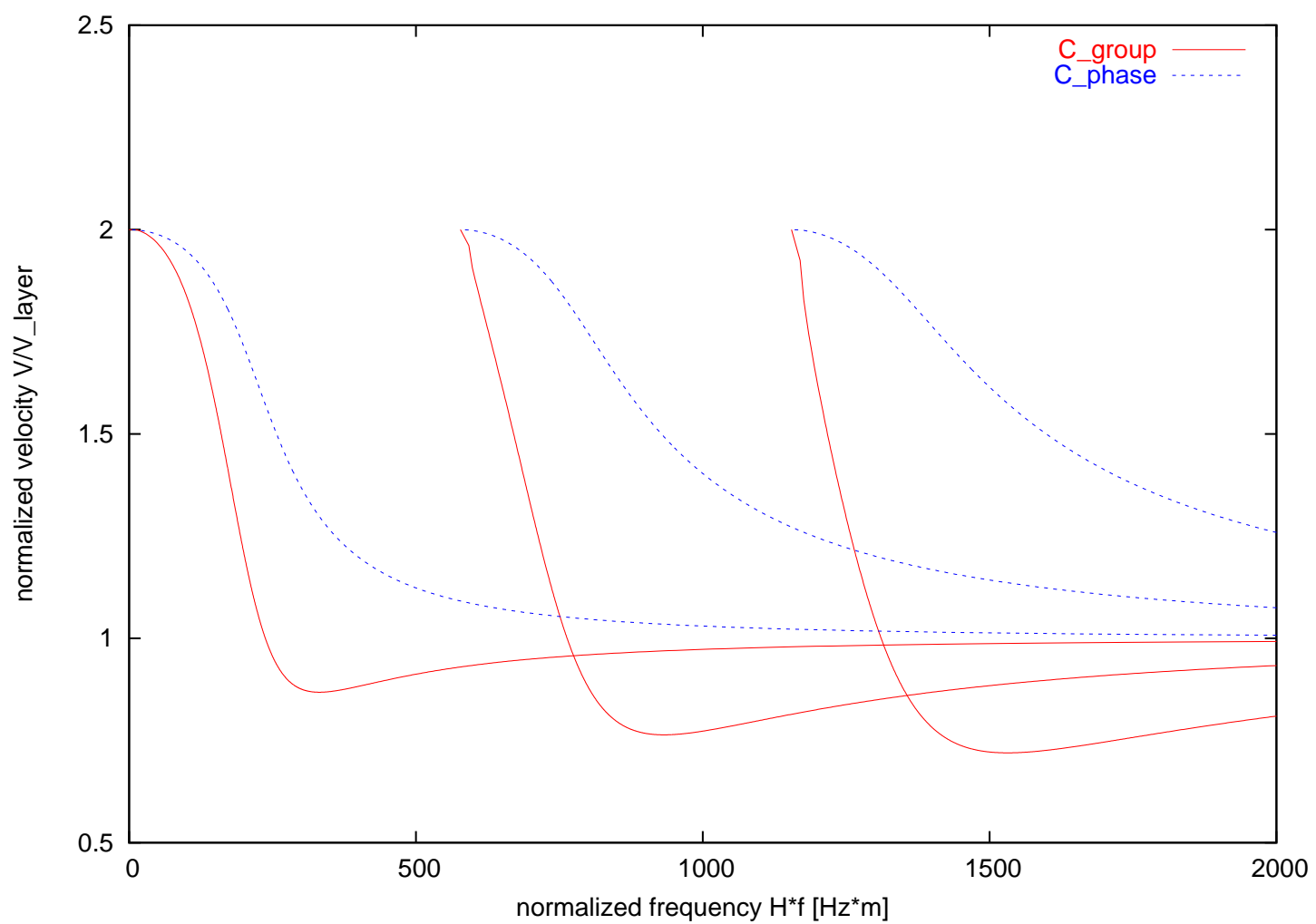

Figure 2.8: Group and phase velocity of the fundamental first and second higher modes of a wave guide (Love waves) normalized by the velocity of the halfspace

\subsubsection{Dissipative and other dispersion effects}

The velocity of propagation becomes frequency dependent due to viscous damping, with increasing velocity for increasing frequency. This is termed abnormal dispersion behavior, and contrasts to the normal dispersion if interface waves generally observed in layered media with seismic velocities are increasing with depth. The amount of dispersion increases $5 \%$ in very dissipative media. This is expressed in terms of the ratio between the frequency dependent velocity change and the elastic velocity of the medium. This can be estimated 
using equation 2.16 after (Kjartansson, 1979).

$$
\begin{gathered}
c=c_{o}\left|\frac{\omega}{\omega_{o}}\right|^{\gamma} \\
\gamma=\frac{1}{\pi} \tan ^{-1}\left(\frac{1}{Q}\right) \approx \frac{1}{\pi Q}
\end{gathered}
$$

For very soft marine sediments quality factors below 10 have been reported and the suggested dispersion is about $10 \%$ for these media. In Sediments with Q factors exceeding 20 the dispersion due to dissipation is insignificant compared to the velocity dispersion of interface waves and wave guides.

Adapting the concept of the effective medium for the steady state pressure equilibrium we will still require to account for dynamic effects in the presence of a composite material, i.e. the influence of pore pressure dissipation and fluid flow regimes in the pore space and many other factors. The fluid effects on wave propagation of porous media has been subject of many theoretical investigations based on and further developed from the Biot model accounting for the stress induced fluid movement in pores. But it was also shown, that most effects are dominant at frequencies higher than the frequency range used in seismic surveying, hence little relevant for measurements discussed in this work. The relevant frequency range for each of the proposed phenomena has been compiled by Mavko (Fig. 2.9). However, it has to

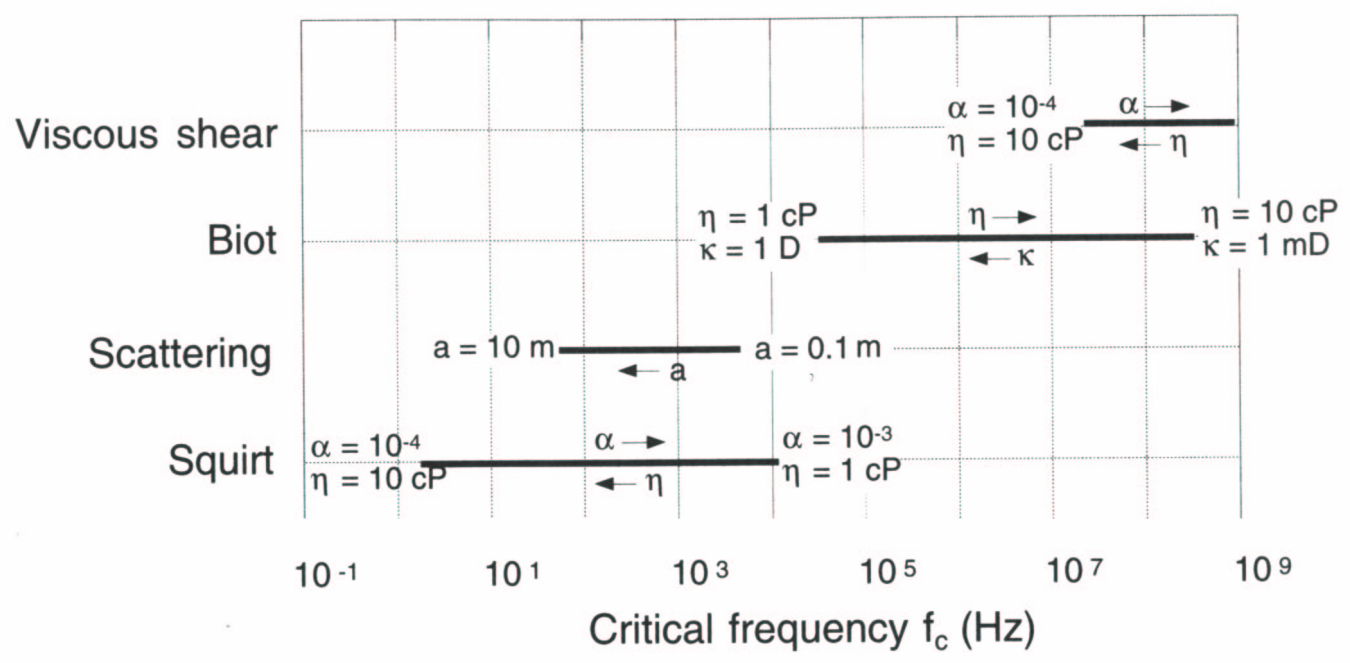

Figure 2.9: Frequency ranges of fluid flow processes in porous sediments (in Mavko, 1998)

be acknowledged, that these mechanism can be of importance when comparing the affect on dispersion from seismic measurements with laboratory measurements and for prediction of dispersion effects at different frequencies. 
Edelman (2002) presented the adaption of Biot's theory for poroelastic media. It is stated, that the poroelastic influence on dispersion and the propagation of interface waves is only relevant at frequencies higher than the seismic frequencies of marine seismic experiments using airgun sources. However, it will be relevant, when comparing the results from in-situ seismic experiments with laboratory measurements which are generally conducted at sonic or ultrasonic frequencies (Edelman, 2002). 


\section{Chapter 3}

\section{Analysis of Dispersive Seismic Waves}

\section{Contents}

3.1 Modeling of Seismic Dispersion . . . . . . . . . . . . . . 38

3.2 Analysis of Dispersive Seismic Wavefields . . . . . . . . . . . 39

3.3 Multi-channel Dispersion Analysis . . . . . . . . . . . . . 40

3.3.1 Fourier Transform and Slant-stacking Methods . . . . . . . . . . . 41

3.3.2 Modified Fourier-Bessel Transform . . . . . . . . . . . . . . . 44

In the previous chapter I have derived that the seismic properties of marine sediments can be described by a laterally homogeneous stratified model of viscoelastic parameters. Subject to the excitation of seismic energy the wavefield exhibits distinct eigenmodes of oscillation, which are related to these model parameters.

In the following three chapters I present the methods of data acquisition and analysis used and further developed within this thesis. The different methods to obtain wave field spectra, which visualize the dispersion of the measured seismic data are discussed in chapter 3 . I begin with the methods to compute synthetic dispersive wave fields from one-dimensional subsurface models representing a simplified marine sediment, in order to analyse and understand the dispersion characteristics. These modeling methods are also used in the inversion procedures in chapter 5, while the acquisition of dispersive seismic wave fields in the marine environment is the topic of chapter 4 . 


\subsection{Modeling of Seismic Dispersion}

We use the theoretical concepts of seismic wave propagation presented in the previous chapter to numerically compute synthetic seismic wavefields. The marine sediment is represented by a viscoelastic model comprised of plane homogeneous layers. The seismic source, generally we use an explosive source, and the one-dimensional earth model uniquely determine the seismic wavefield with all dispersive wave types, including interface waves as well as guided waves and leaky modes. This enables us to compute the dispersion characteristics of the idealized medium and to study the variation of the dispersion characteristics for variations of the model and acquisition parameters.

Various computer algorithm have been published, which can perform this task. The computations of normal modes can be obtained using the Thomson-Haskell method (Haskell, 1953; Thomson, 1950), which has been further developed by Schwab and Knopoff (Schwab \& Knopoff, 1972). Other methods are based on the superposition of spherical harmonic solutions of the numerically integrated wave equation (Friederich \& Dalkolmo, 1995, e.g.), or the propagation of the displacement and stress fields across the interfaces of a stack of homogeneous layers in form of efficient propagator matrix formulations (Fuchs \& Müller, 1971; Wang, 1999). A discussion of advantages and disadvantages of selected algorithm can be found in (Buchen \& Ben-Hador, 1996).

A summary for each of the algorithms used throughout the thesis is given in the appendix. Here I will just mention their key features. The mode summation algorithm FLSPHER, developed and provided by W. Friederich in personal communication, enables to compute eigenfunctions as well as sensitivity kernels and solutions to the dispersion relation with respect to frequency for one-dimensional spherical or flat earth models. We can therefore produce the dispersion curves, the corresponding eigenfunctions and the sensitivities with respect to changes of model parameters. The main advantage of this algorithm is the possibility to study the distinct modes and their contribution to the wavefield separately.

For the computation of synthetic wavefield spectra I used the wavefield algorithm presented by Wang (1999). This code enables to compute the complex wavefield spectra in the frequency slowness domain but ommit the computation of seismograms. The seismic wavefield excited by the input source signal is solved analytically within the homogeneous layers and propagated across the interfaces. Therefore, the computed seismic wavefield only contains solutions related to the specific source and receiver depth. Since the solution is obtained in the p-f-domain and the time consuming step of computing seismic waveforms is ommitted, it is an effective and fast algorithm to compute full wavefield spectra.

A finite difference algorithm is used in case of a laterally varying medium (Bohlen, 1998). This enables to produce snap shots of the wavefield separated into compressional and shear wave contributions. Another advantage of this method is the identification and propagation of dispersive wave arrivals at specific receiver locations . 
The combination of these modeling tools enables us to investigate and illustrate the characteristic features of dispersive wave propagation in marine sediments. Furthermore it enables to compare the predicted wavefield for a certain subsurface model with the measured seismic data. Yet, in order to facilitate this comparison, I will discuss the methods to analyze and visualize the dispersion of measured seismic wavefields.

\subsection{Analysis of Dispersive Seismic Wavefields}

I will start with the description of common data analysis methods, since the choice of method of analysis of dispersive waves puts some constraints and requirements on the acquisition and processing of the data. Here I will just mention a few commonly used methods in order to discuss the advantages and disadvantages of the method proposed and used in this work as described in section 3.3.2. The application of a full wavefield transform imposes the requirement of adequate spatial and temporal sampling. The implication of these requirements on the data acquisition and processing are then discussed.

The dispersion of seismic wavefields is expressed by the frequency dependent variation of the phase velocity of seismic wavelets, which are determined by the amplitude distribution in time and space. Therefore it seems most natural to determine the dispersion characteristics by transforming the wavefield from the time to frequency and from space to velocity domain (i.e. wave number $k$ or slowness $p$, since phase velocity $\left.c=\frac{1}{p}=\frac{\omega}{k}\right)$. To enable the use of the two dimensional Fourier transform of time series, the temporal sampling $\Delta t$ and the distance between two adjacent seismograms $\Delta x$ has to be adequate according to the Nyquist theorem $f_{N y q}=1 / 2 \Delta t$ and its equivalent theorem in space.

$$
c \geq c_{\min }=\frac{\omega}{k_{N y q}}=2 \pi f \Delta x
$$

The Nyquist-Frequency $f_{N y q}$. determines the maximum frequency allowed in the time series which can be resolved unambiguously and equivalently the smallest resolvable phase velocity $c_{\min }$ is determined by the Nyquist wavenumber $k_{N y q}$. Since these criteria can not always be fulfilled, alternative methods to determine the dispersion of seismic waves have been developed.

Since the distribution of seismological stations is sparse, the wavefield transform methods can not be applied. Analysis methods to derive the dispersion from time domain data were first developed by early seismologists investigating the dispersion of strong amplitude surface waves generated by earthquakes. Two methods are still commonly used, firstly the multiplefrequency-filtering-technique (MFT) method to derive the group velocity dispersion from

\footnotetext{
${ }^{1}$ While the phase velocity is related to the angular frequency $\omega$ the term frequency in the text refers to the frequency $f=\omega / 2 \pi$.
} 
a single seismogram and secondly the phase-shift or phase-difference method. The phase velocity is obtained from the phase spectrum of the cross-correlation of two seismograms with a remaing ambiguity of the factor of $2 \pi$ (Seidl \& Müller, 1977). The phase-difference method requires that the wave train used to measure the phase difference corresponds purely to one mode of the surface wave. Due to the large distances from earthquake and due to the nature of the source this is adequate in many cases in seismology, but with the short distances used for surveys with shallow targets on land as well as in the marine environment, this is generally not the case. Despite of the fact, that the method has become popular for geotechnical applications under the name of Spectral Analysis of Surface Waves (SASW), the limits with respect to identification of the modes had to be acknowledged in many cases.

The application of the MFT-method to a single seismogram involves the application of narrow-band frequency filters to the time series and to interpret the arrival time with respect to the source-receiver distance of the signal as group velocity (Dziewonski et al., 1969). Since the width of the frequency filter and the length of the time window can not be chosen independently the resolution in the so-called Gabor-matrix display of frequency versus group velocity is always very limited. This methods can identify small variations of the dispersion relation from the expected values. Again, while this is useful in seismology, the variability in dispersion of shallow targets is often high and the multi-modal dispersion characteristic of the sub surface cannot always be resolved with this method.

Despite of the fact, that these methods commonly used in seismology are of limited use in the marine environment, we do not necessarily suffer from the lack of seismograms in active marine seismic experiments. We will therefore now focus on the analysis of multi-channel analysis methods which enable to transform the seismic data into the frequency-wavenumber $(f-k)$ or frequency-slowness $(f-p)$ domain.

\subsection{Multi-channel Dispersion Analysis}

The algorithm commonly used in dispersion analysis are usually based on the slant-stacking method as originally described by McMechan and Yedlin (McMechan \& Yedlin, 1981). An offset-dependent time shift is applied to each seismic trace before stacking to obtain a single trace with large amplitudes, if the phases of the seismograms are in phase, hence propagated with the phase velocity corresponding to the applied time shift. This is performed subsequently for the phase velocity range of interest, obtaining one transformed time series for each slowness (1/velocity) value. Seismic signals out of phase, i.e. traveling with different phase velocity, do not contribute to the stacked section. A 1-D-Fourier transform with respect to time is applied to the slowness section to obtain a frequency-slowness spectrum.

Several modifications of this slant-stack method in either time or frequency domain have been applied to perform dispersion analysis (Klein et al., 2000; Park et al., 1999; Bohlen 
et al., 2003; Bohlen et al., 1999; Xia et al., 2000; Beaty \& Schmitt, 2000). This method is robust and suitable to visualize the dispersion characteristics of the data set in the slowness spectra.

These methods represent a two dimensional transform with respect to time and space. The transformation of the seismic wavefield $u(x, t)$ can be formulated in terms of the 2-d Fourier transform of the seismic source $S(k, \omega)$ and a "dispersion relation" $D(k, \omega)$ with $k$ and $\omega$ denoting the horizontal wave number and angular frequency respectively (McMechan \& Yedlin, 1981). In fact, the term $D(k, \omega)$ is not strictly a disperison relation but corresponds to the Rayleigh determinant in case of the analysis of Rayleigh wave propagation and therefore yields the dispersion relation for the condition $D(k, \omega)=0$.

$$
u(x, t)=\frac{1}{2 \pi} \iint \frac{S(k, \omega)}{D(k, \omega)} e^{i(k x-\omega t)} d \omega d k
$$

which transforms to

$u^{\prime}(p, \tau)=\int u(x, \tau+p x) d x=\iint \frac{S(k, \omega)}{D(k, \omega)} \delta(k-\omega p) e^{-i \omega \tau} d \omega d k=\int \frac{S(\omega p, \omega)}{D(\omega p, \omega)} e^{-i \omega \tau} d \omega$

The contribution to the wavefield is large, when the term of the dispersion relation is fulfilled and therefore $D(\omega p, \omega)$ is small, and the source signal contributes energy at the frequency of concern. Therefore large amplitudes in the $p-f$-domain represent dispersive modes of the measured seismic wavefield. This method can be applied using standard processing tools, but the sampling criteria in both spatial and time domains need to be fulfilled to avoid artifacts from aliasing. In practice several methods to be discussed in the following sections have been applied to the time domain data.

\subsubsection{Fourier Transform and Slant-stacking Methods}

The method to compute the slowness-frequency spectra suggested by (McMechan \& Yedlin, 1981) consists of a linear radon transform into the $\tau-p$ domain representation before applying a Fourier transform with respect to the time $(\tau)$ dimension as described previously. Park et.al. 1998 stated, that resolution of the $\tau-p$ domain data is enhanced by slant stacking in the frequency domain. Hence, the 1-D Fourier transform with respect to time is applied before stacking traces of common slowness with offset and slowness dependent phase shift $\left(e^{-i \omega p x}\right.$, $p=$ slowness, $\mathrm{x}=$ offset,$\omega=2 \pi f$ ).

$$
\begin{gathered}
\widetilde{u}(r, f)=\int_{u} u(x, t) e^{i \omega t} d t \\
U(p, f)=\sum_{l=1}^{N} \widetilde{u}\left(x_{l}, f\right) e^{-i \omega p x}
\end{gathered}
$$


We thus obtain the spectral coefficients $U$ of the seismic wavefield in the slownessfrequency domain. This slowness-stacking method has been implemented as supplemental tool to the Seismic Unix package (s. appendix D.2.2).

Despite of the theoretical equivalence of the application of the slant stack in time or frequency domain, in practice the results from the frequency domain are superior in terms of resolution in frequency. This is predominantly due to the advantage that the phase correction in the frequency domain is not restricted to certain sampled values and can be applied continously. In the time domain, the stacking of amplitudes often requires an interpolation of the amplitudes between samples. This can lead to inaccuracies and is easily avoided by performing the stacking in the frequency domain.

The resolution in slowness domain $\Delta p$ should be comparable for both methods. It is determined by the width $L$ of the offset window of the seismic gather (Forbriger, 2003a)

$$
\Delta p \approx \frac{1}{f L}
$$

Hence, the length of the receiver spread in case of a common-shot-gather determines the resolution in the slowness domain. If the length of the spread is sufficiently long, we may limit the spread and investigate different offset ranges. Thus we obtain the dispersive seismic wavefield at different offset ranges of the same length, and can compare these to obtain information on the lateral variability of the dispersive wavefield.The potential of these local spectra is to be able to depict lateral variations of the sediment properties along the profile. If the profile is long enough to be divided into reduced offset windows $L_{i}$ with sufficient length to obtain adequate resolution in the $p-f$-spectra, we can derive shear wave velocity profiles for several locations along the profile, respectively to the situation at the center of the offset window $L_{i}$. In practice, we apply a gauss-shaped offset-window taper of width $L_{i} \simeq 200 \mathrm{~m}$, which denotes that the amplitudes are reduced to $1 / e$ at 100 distance to each side of the center $x_{c_{i}}$ of the window. The center of the window function is than moved at 50-100 m intervals ( $x_{c_{i+1}}=x_{c_{i}}+50$ ) along the profile to obtain the gradual variation of seismic parameters of the sub surface along the profile (Bohlen et al., 2003). Schneider proposed a method to produce local $\omega-k$ spectra by 2-D Fourier transform of narrow bandlimited wavenumber slices (Schneider, 1993). After reordering for common offset ranges a set of local spectra is obtained. This is equivalent to the application of an offset-window taper to the time domain data prior to the slowness-stacking method just described.

In order to depict the trade-off between resolution due to the limited offset range I show two synthetic examples, where the spectral coefficients are computed for a long offset and a limited offset gather (Fig. 3.1). While the resolution of the dispersion curves decrease with limited offset, the amplitude distribution of the slowness spectrum generated from the synthetic seismograms still reveals the dispersion characteristics of the medium. Generally the influence of seismic attenuation has similar effects on the amplitude spectra, therefore 


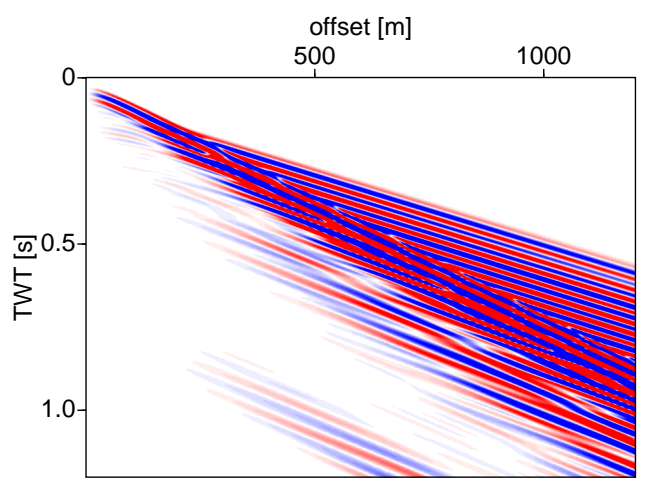

Synthetics (OBS) $1200 \mathrm{~m}$

(a) OBS-Gather

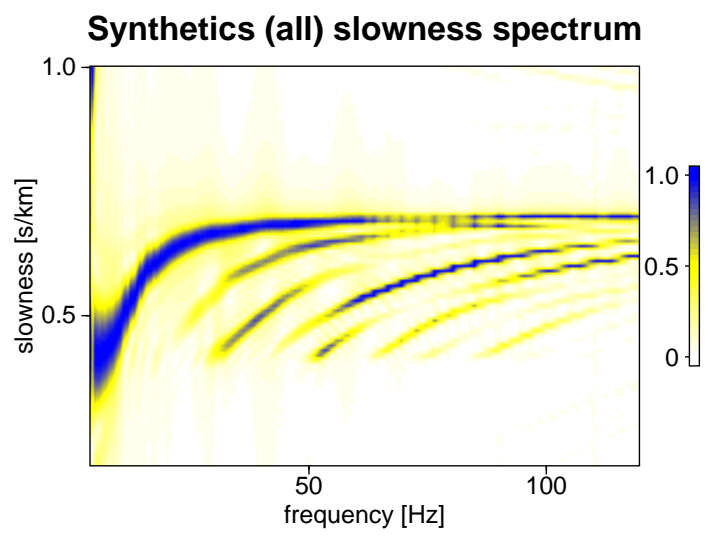

(c) slowness spectrum of a.

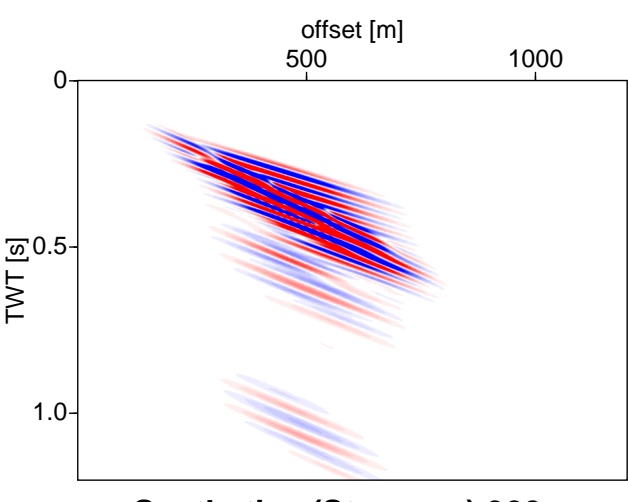

Synthetics (Streamer) 300m

(b) Streamer-Gather

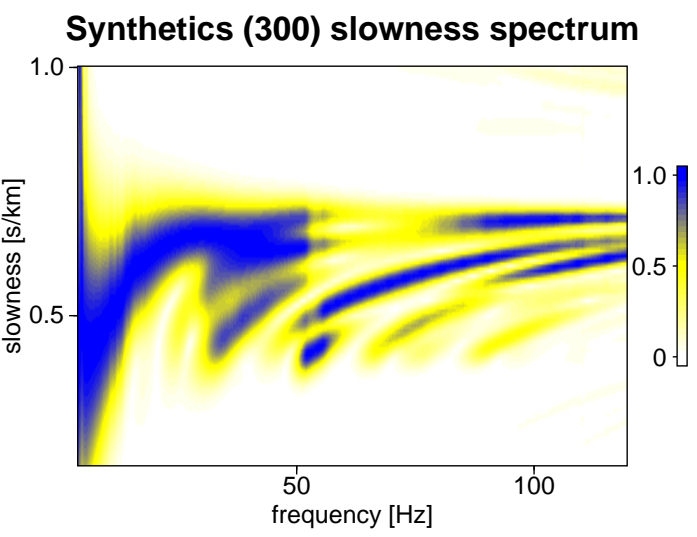

(d) slowness spectrum of $b$

Figure 3.1: Comparison of seismogram gather and slowness spectra for full (OBS) and limited (Streamer) offset ranges. 
we expect to loose information on the seismic parameter Qs and Qp with offset limitations. However, if the resolution degrades further, the interpretation of the modes may be ambiguous which may cause problems for the choice of a start model in the inversion process to be discussed in chapter 5 .

\subsubsection{Modified Fourier-Bessel Transform}

The formulation of the slant-stack transform is based on the assumption of plane waves. Therefore the spectral coefficients obtained by this transform are based on cartesian coordinates while the synthetically computed spectral coefficients are obtained for a spherical coordinate system. Secondly, despite of the existance of an inverse transformation to the slant-stacking algorithms, in practice it is often problematic to reproduce the seismic wavefield by application of the forward and inverse transforms.

For the purpose of an inversion of the measured spectral coefficients a comparable formulation is required. Forbriger (2003a) suggests an algorithm based on a modified FourierBessel transform to perform the wavefield transform to produce the slowness spectra, since this algorithm can be used to calculate true amplitude spectral coefficients which can reproduce the recorded data in terms of coefficients to Bessel-functions, i.e. relate to the spherical coordinate system. Secondly the inverse transform exists and is more stable than the inverse tranform of the slant stack algorithm.

In order to proceed with the inversion in the frequency-slowness domain we need to apply such a wavefield transform which computes complex (true amplitude) spectral coefficients to represent the seismic wavefield. The seismic wavefield recorded at distance $r$ from a cylindrically symmetric source which excites a seismic wavefield propagating through a laterally homogeneous layered medium can be described by the Bessel-function expansion

$$
\widetilde{u}_{z}(\omega, r)=\int_{0}^{\infty} G(\omega, p) J_{0}(\omega p r) p d p
$$

where the displacement $u$ is given by the slowness $(p)$ integral over the spectral coefficients $G$ of the vertical component and the Bessel function $J_{0}$. Forbriger shows, that using the Hankel function $H_{\eta}^{(2)}$ describes the outgoing wavefield only and reduces artificial noise in the wavefield transform (Forbriger, 2003a). The spectral coefficients $G(\omega, p)$ are obtained from the measured displacement $u(\omega, r)$ of the $N$ seismograms at positions $r_{l}$.

$$
G=\frac{\omega^{2}}{2} \sum_{l}^{N} \tilde{u}_{z}\left(\omega, r_{l}\right) H^{(2)}\left(\omega p r_{l}\right) r_{l} \Delta r_{l}
$$

Therefore I used this method to calculate the full complex spectral coefficients of the measured seismic wavefield, as required for the inversion procedured to be discussed in chapter 
5. The application of approriate scaling and tapering of the input data prior to the transformation reduces the artifacts in form of side-lobes significantly. This applies to any of the full wavefield transforms, but is of special interest for the subsequent use of the transformed wavefield spectra for the inversion, since the artifacts and amplitudes related to the wavefield are not distinguished in the inversion. The appropriate scaling is usually an offset $r^{\alpha}$ dependent amplitude enhancement with the exponent $\alpha \approx 1.5$ in order to compensate the geometrical spreading of the interface wave. Tapering is applied in time as well as in the offset domain.

Both, the frequency domain slant stack and the modified Fourier Transform should produce comparable results as indicated by the data examples in fig. 3.2.
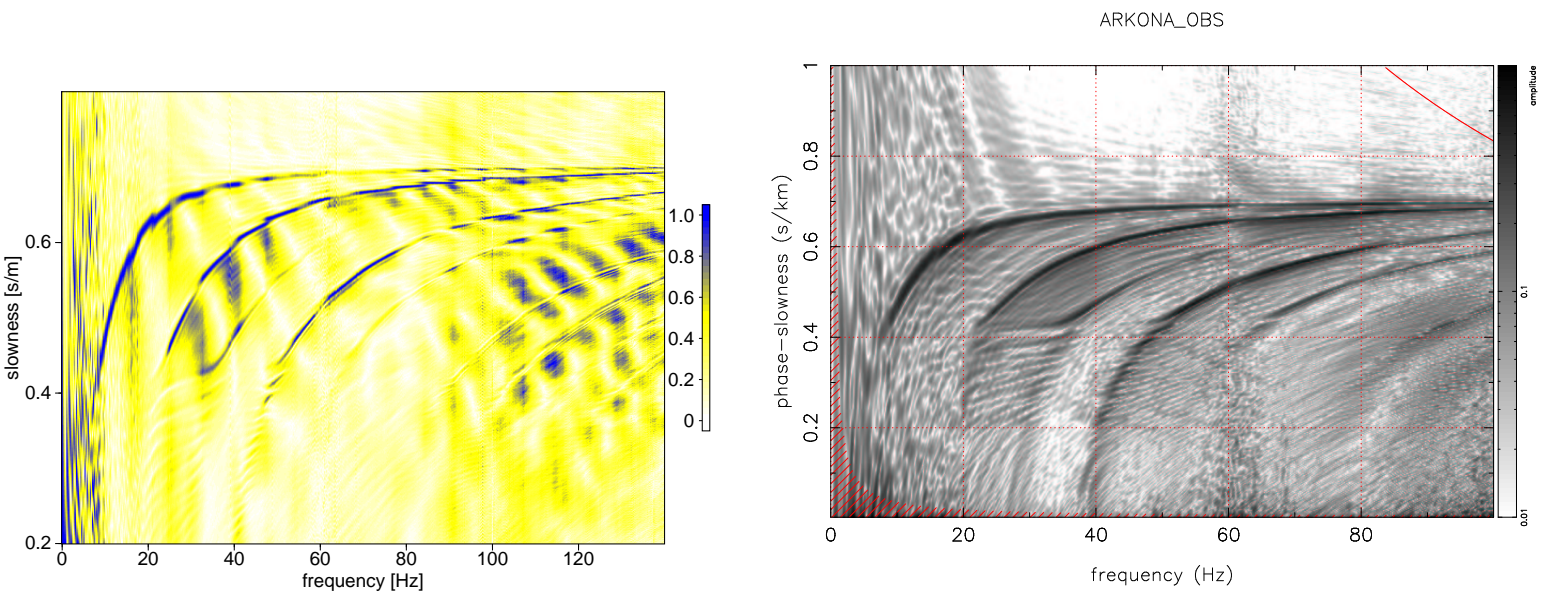

Figure 3.2: Comparison of the wavefield spectra obtained by the application of a frequency domain slant stack (left) and a modified Fourier-Bessel transform (right) of a real data set further discussed in chapter 8 . 


\section{Chapter 4}

\section{Acquisition of Dispersive Wavefields}

\section{Contents}

4.1 Determination of the Acquisition Parameters . . . . . . . . . . . 48

4.1.1 Determining the Frequency Range of Interest . . . . . . . . . . . 48

4.1.2 Energy Distribution with Depth of Scholte Waves . . . . . . . . . 50

4.2 Excitation and Registration of Interface Waves . . . . . . . . . . 50

4.2 .1 Seismic Sources at the Sea bed . . . . . . . . . . . . . . 50

4.2.2 Seismic Receivers at the Sea Bed . . . . . . . . . . . . . . 52

4.3 Seismogram Gathers in Different Acquisition Geometries . . . . . . . 53

4.3.1 Obeying the 2-D Sampling Theorem in Time and Space . . . . . 53

4.3.2 Common-Receiver-Gather $(\mathrm{OBS} / \mathrm{H}) \ldots \ldots \ldots . \ldots 54$

4.3.3 Common-Shot-Gather $(\mathrm{OBC} /$ Streamer $) \ldots \ldots \ldots$

The application of the wave field transform discussed in the previous chapter puts additional requirements on the acquisition parameters, which I will discuss in this chapter in respect to the methods used for the excitation and recording of dispersive seismic waves. 


\subsection{Determination of the Acquisition Parameters}

In order to define an acquisition and analysis procedure to measure and invert dispersive interface waves I continue with numerical investigations on the energy distribution of Scholte waves within the water column. This is necessary, since we wish to excite and/or record the seismic wavefield in the water column rather than in contact to the sea bed, in order to develop a towed acquisition system capable to record Scholte wave dispersion in shallow marine environments. Secondly, the frequency range of interest must be determined. Thus the frequency range of the transition from the propagation of the interface wave with Scholte wave velocity to Rayleigh wave velocity, which corresponds to the Airy phase of the dispersive mode, must be observed in the seismic experiment.

\subsubsection{Determining the Frequency Range of Interest}

The dispersion curves of laterally homogeneous elastic media are calculated effectively by normal mode algorithm, in this case we used the program FLSPHER provided by W. Friederich in peronal communication (Friederich \& Dalkolmo, 1995). We computed dispersion curves for a reference model shown in fig. 4.1a. The dispersion curves for the model are plotted in fig. 4.1b. and for source and receiver depth of 10 and $20 \mathrm{~m}$ respectively, we obtain the amplitudes of the modes indicated as error bars. The fundamental mode approaches the Scholte wave velocity at the highest frequency, while the higher modes tend to lower slownesss values (higher velocities) at this frequency range. From fig. 4.1c, where the variation of the dispersion curve with variations for the sediment shear wave velocity is plotted for the first three modes, we can see, that at higher frequencies the higher modes approach the lowest shear wave velocity of the model. However, the amplitude distribution in b) given by the size of the error bars shows no amplitudes for these modes at high frequencies. Only the first higher mode has some amplitude at frequencies form 7-15 Hz. For higher frequencies all the energy is found in the acoustic modes propagating at water velocity (corresponding to slowness of $0.66 \mathrm{~s} / \mathrm{km})$.

In fig. 4.1d) the sediment layer thickness was subject to variations in the range from 1 to $10 \mathrm{~m}$. For the sediment thickness of less than $4 \mathrm{~m}$ the fundamental mode follows the slowness corresponding to the Scholte wave velocity of the lower layer. Both, the shear wave velocity as well as the Scholte wave velocity for the model without any surfical sediment layer are marked in the figure.

Note the influence of variations of the shear wave velocity in the sediment layer on the dispersion curves in fig. 4.1c) compared to the influence of the variation of the thickness in the sediment layer shown in fig. 4.1d). While at frequencies lower than $15 \mathrm{~Hz}$ the variation of the position of the dispersion curve of the fundamental mode is hardly distinguishable for variations of sediment thickness or shear wave velocity, the position of the first higher mode 
does vary significantly.

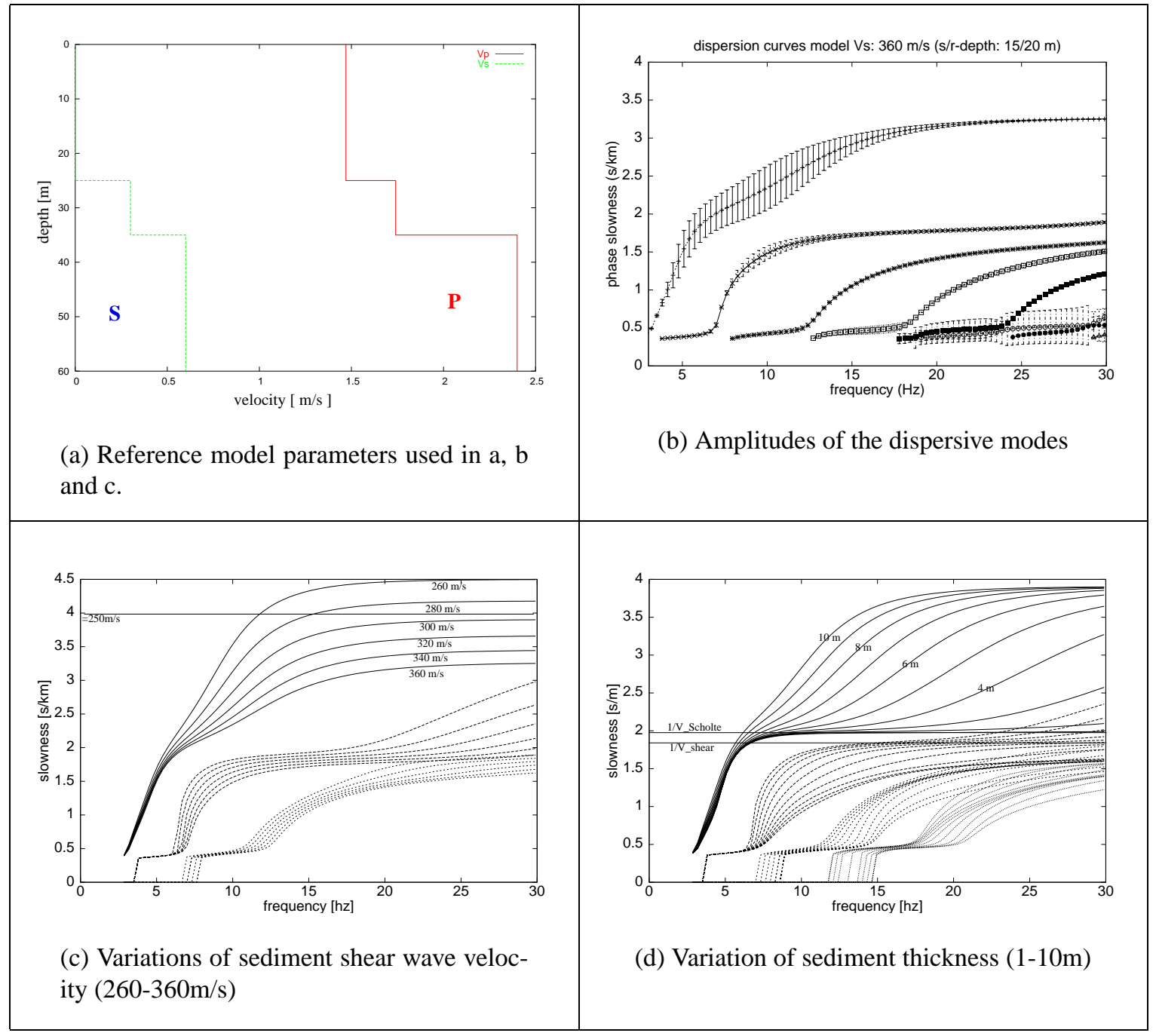

Figure 4.1: The variation of dispersion curves with sediment properties for the model given in a) (top left) is shown for variations in shear wave velocity (bottom left) and sediment layer thickness (bottom right). The errorbars of the dispersion curves (top right) represent the amplitude coefficients relating to the obtainable amplitudes for excitation and recording at 15 $\mathrm{m}$ and $20 \mathrm{~m}$ depth corresponding to a deep-towed streamer experiment.

I conclude that the variations of the model parameters cause variations in the fundamental Scholte mode in the frequency range of 5 to $30 \mathrm{~Hz}$ and with significant amplitude levels for the fundamental mode in this range. Therefore we can expect to excite Scholte waves with 
significant dispersion characteristics with a towed air-gun system. Measurements in the Kiel Bay area with surface towed air-gun sources and OBH receivers have also been successful in recording Scholte wave energy with significant dispersion characteristics (see chapter 6).

\subsubsection{Energy Distribution with Depth of Scholte Waves}

The energy distribution of the Scholte modes in the water column can be calculated from the eigenfunctions of the displacement components of the normal modes. The seismic wavefiled can be described by superposition of normal modes which is commonly used to calculate synthetic seismograms. The advantage of this approach to compute the seismic wavefield is, that we can investigate the eigenfunctions of each mode and compute the energy from the displacement vectors of the components. Fig. 4.2 shows an example of the energy distribution with depth for selected frequencies $(3-15 \mathrm{~Hz})$ of the fundamental (red) and first higher mode (blue) calculated for a subsurface model comprised of a soft (mud) layer overlaying sediments (sands or glacial till) commonly found in the Baltic sea. The exponential decay away from the water-sea floor boundary is frequency dependent. While the energy of low frequencies is still significant near surface, amplitudes of higher frequencies decay more rapidly. In order to investigate the dispersion characteristics of Scholte waves we need to record in the appropriate frequency range, hence we need to record the wavefield close to the sea bed in order to extend the frequency range.

\subsection{Excitation and Registration of Interface Waves}

From section 2.5 it is clear that the maximum amplitudes of interface waves is observable and excitable directly at the interface. I will therefore start with the discussion of using seismic sources and/or receivers directly on the sea bed. Following these two sections I will discuss the implification of the sampling theorem on the data acquisition before I describe the potential and disadvantages of the acquisition geometries used to obtain the seismic data presented in this work.

\subsubsection{Seismic Sources at the Sea bed}

The use of seismic sources operating at the sea floor is not proposed, due to the numerous difficulties arising in the operation of such a system. Several investigations in developing seismic sources have been published (Theilen \& Pecher, 1991; Davis, 1996; Stoll \& Bautista, 1994b).

Usually a sledge mounted source was deployed and towed along the sea floor in an stopand-go manner. This implies a very slow movement of the ship, in order to minimise the drag 


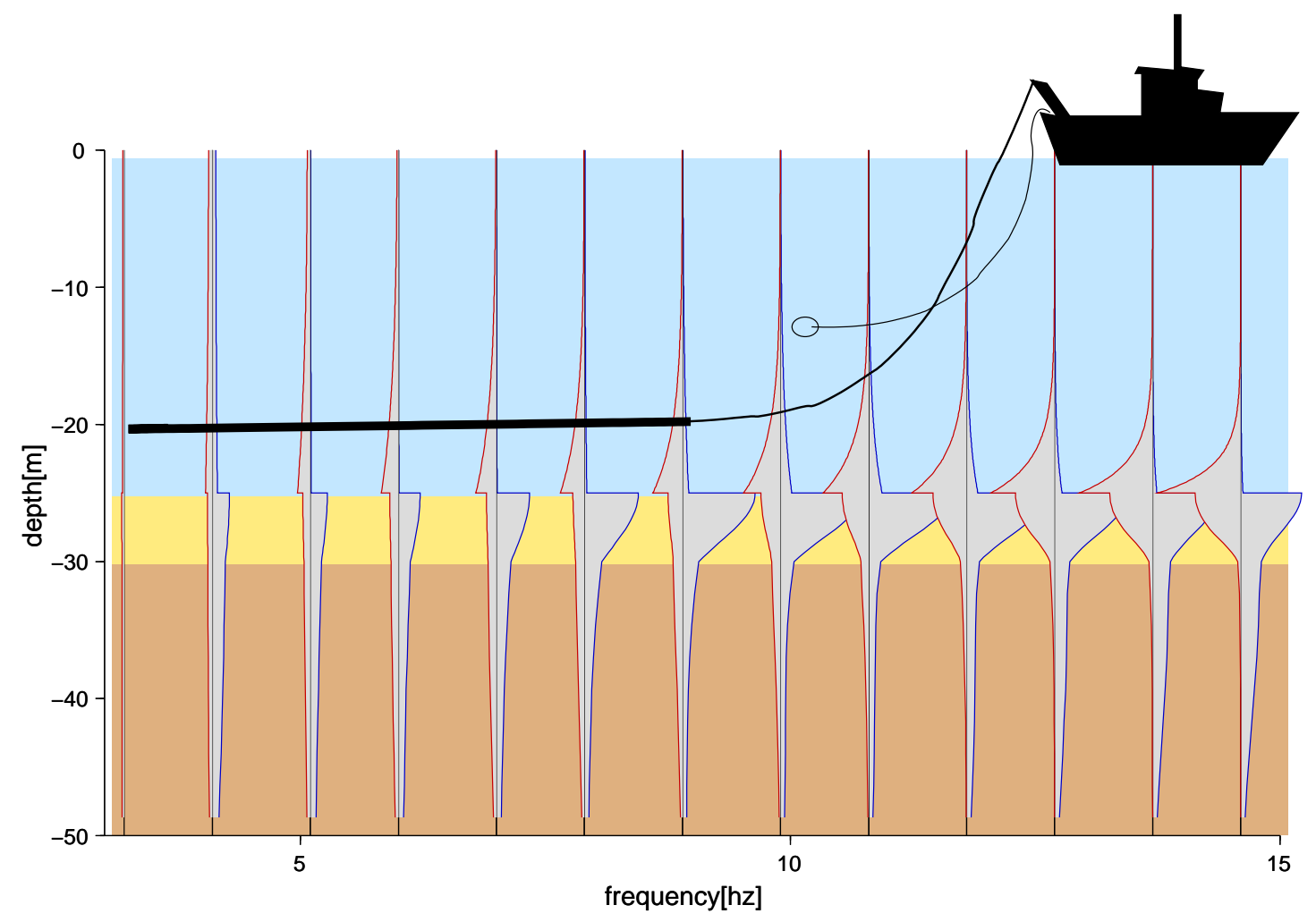

Figure 4.2: The variation of the energy of the eigenfunctions with depth is displayed for discrete frequencies of the fundamental mode (red, amplitudes to the left) and the second mode (blue, amplitudes to the right) in the frequency range from 3 to $15 \mathrm{~Hz}$. The exponental decay of the energy in the water column is more dominant for the high frequencies, hence the frequency band is increased by towing the acquisition system close to the sea bed. Furthermore is the information at higher resolution enhanced if higher modes are included, since these dominate at high frequencies. 
on the system. Secondly difficulties arise with mechanical parts in the vicinity of a suspensions of mud, which naturally occurs during operation in soft sediments, besides the requirement to transport the required energy from the vessel to the sea bed. Hence, the deployment of the seismic source at the sea bed encounters many difficulties, but more importantly, requires very slow ship speeds and therefore limits the coverage.

However, if investigations of several interface types, e.g. Love wave dispersion, were to be investigated, a sea floor operating source must be used. Since this in not required for P-SV polarised interface waves, Scholte wave energy can be excited with sufficient energy using surface towed air-gun sources and we propose to use air-gun sources at the surface or towed at intermediate depth, since this is capable to excite Scholte wave energy and source repeatability is acceptable.

The operating of a source system at the sea floor could be advantageous, if an autonomous source vehicle could be used. Here the energy requirement would remain the main problem to be addressed.

\subsubsection{Seismic Receivers at the Sea Bed}

While in terms of aerial coverage and efficiency decoupling from the sea bed for both, source and receivers, would be advantageous, we have to consider that the decrease in energy, as represented in the eigenfunctions of the specific dispersive modes, applies twice, for source and receivers respectively. Secondly, the use of seismic sensors at the sea bed enables not only to record the pressure field, but additional registrations of the particle motion at the bed with 3-component geophones help to understand, identify and separate the contributions of the dispersive seismic wavefield. Since autonomous seismic sensor stations are available we can use stationary ocean bottom sensors $(\mathrm{OBH} / \mathrm{OBS} / \mathrm{OBC})$ with a towed seismic source in a kind of walk-away seismic experiment. While this acquisition layout inherits several limitations, it is still more efficient and easy to operate than ocean bottom sources. In order to avoid confusion with a vertical-seismic-profiling method also denoted as walk-away experiment, I will denote this layout as stationary-receiver method, despite of the fact that it is irrevelant whether the source or receiver is kept stationary.

However, the application of ocean bottom seismometer (OBS) stations requires to account for the coupling of the seismic sensors to the sea bed, if particle motion is to be observed. This is even more important if the OBS is to be used at different locations or several OBS stations are to be used, since every deployment of the sensor might produce different sensor coupling.

The use of autonomous sensor stations requires the data to be stored, which might limit the acquisition parameters in terms of time sampling and recording length. Due to the rapid development of fast and large storage media and A/D-converters today, this is rarely a limitation. 
Another factor arising from the use of stationary autonomous sensors, is the requirement to accurate absolute positioning of both the receiver as well as the source during the seismic experiment, in contrast to a towed acquisition system, where the source-receiver geometry remains approximately constant with regard to the ship position. Therefore the positioning of the seismic source must be achieved at high accuracy, e.g. using differential GPS and/or laser tracking systems. The position of the ocean bottom sensors must be assessed, e.g. by additional transponder or seismo-acoustic measurements or special data analysis.

Hence, we have to consider if the superior quality and information obtainable by multi component OBS outweighs the expense and limitation in progress and efficiency.

\subsection{Seismogram Gathers in Different Acquisition Geome- tries}

The application of the wavefield transform requires a group of seismograms with variation in the source-receiver distance (offset). The offset variation can be achieved either by moving the source or the receiver or both, each of which solutions has practical advantages and disadvantages in terms of the acquisition and processing. Limitations to the configuration arise from the requirement to sample the seismic wavefield adequately with respect to time and space.

\subsubsection{Obeying the 2-D Sampling Theorem in Time and Space}

In order to apply the two dimensional wavefield transform we require to sample the seismic wavefield at sufficiently small intervals in space and time over an sufficiently long distance and period in time. This simple rule has many consequences for the acquisition of marine seismic data, which is suitable to be transformed into the $f-p$ - domain.

The time sampling is limited by the registration hardware in terms of the capability of the A/D-converter. Secondly, the amount of data storage required per seismic gather is dependent on the number of samples per record length. Since the frequency range of interest for either of the dispersive wave types is well below the acoustic limits the time sampling is generally no problem, but sometimes the storage capacity can be limited, when recording systems are to be deployed autonomously on the sea bed.

The time period required to record the full seismic wavefield depends on the seismic wave velocities of the sub surface and the maximum distance between the seismic source and receivers $X_{\max }$. Hence, we can formulate the rule of thumb to obtain the minimum recording length $T_{R e c}$,

$$
T_{\text {Rec }}=\frac{X_{\max }}{V_{\min }}
$$


which is also the lower limit of the shot-to-shot rate. The velocity $V_{\min }$ must be compared with the lowest velocity of propagation of interface waves in the medium, which corresponds with the Rayleigh wave velocity of the medium. Therefore, a lower limit approximation is found by using 0.8 times the lowest shear wave velocity in the sub surface model, since the factor between the Rayleigh wave and shear wave velocity ranges from 0.81 to 0.98 for sediments with realistic Poisson's ratio. Hence we obtain two relations concerning the required recored length equivalent to the minimum shot-rate and the maximum offset as well as the shot-to-shot distance (3.1) related to the lowest shear wave velocity $c_{\min }$ resolvable in the medium.

This will constrain the layout of stationary-receiver seismic experiments, when either receiver or source are deployed stationary at the sea floor while the other is towed behind the vessel, since then the shot-to-shot distance $\Delta x$ is related to the towing speed of the vessel. In conjunction with the sample criterion 3.1 we obtain the relation between the ship speed $V_{\text {ship }}$, the maximum offset $X_{\max }$ and the lowest resolvable seismic velocities of $V_{\min } \geq 0.8 c_{\min }$.

$$
c_{\min }^{2}=5 \pi f_{\min } X_{\max } V_{\text {ship }}
$$

For a marine seismic survey with conventional $4 \mathrm{~Hz}$-sensors $\left(f_{\min }\right)$ assuming the minimum ship speed required to keep the vessel manouverable at $1 \mathrm{kn}$, this implies, that the minimum shear wave velocity resolvable at $500 \mathrm{~m}$ distance is $\mathrm{V} \sim 125 \mathrm{~m} / \mathrm{s}$ (Fig 4.3). The offset window $X_{\max }-X_{\min }$ used for the wavefield transform coincides with the window length $L$ and therefore determines the slowness resolution of the wavefield transform.

For very soft marine sediments with shear wave velocities in the range of $10 \mathrm{~m} / \mathrm{s}$ the requirement on the sampling interval is of $\Delta x<10-50 \mathrm{~cm}$. This renders it insensible to use the seismic stationary-receiver experiment for such sediments. It would require more than 8.5 hours to cover a $300 \mathrm{~m}$ long survey line if shear wave velocities of $10 \mathrm{~m} / \mathrm{s}$ are to be resolved. Here the use of a receiver array is more sensible, but requires the receiver array to comply to the sampling criterion of $10-20 \mathrm{~cm}$. Hence, the measurement of Scholte waves remains problematic in case of very soft marine sediments.

The effect of aliasing is visualized in the data set shown in figure 4.4. The signal energy reappears at small slowness values when the energy would normally appear above the aliasing line. Hence, a dispersion curve which meets the aliasing line at a certain frequency reappears in the lower part of the image at this frequency. Secondly aliased energy appears in form of the aliasing line itself.

\subsubsection{Common-Receiver-Gather (OBS/H)}

Using a towed source in conjunction with a stationary receiver deployed at the sea bed implies the limitation on velocity resolution as discussed in the previous section. This could be 


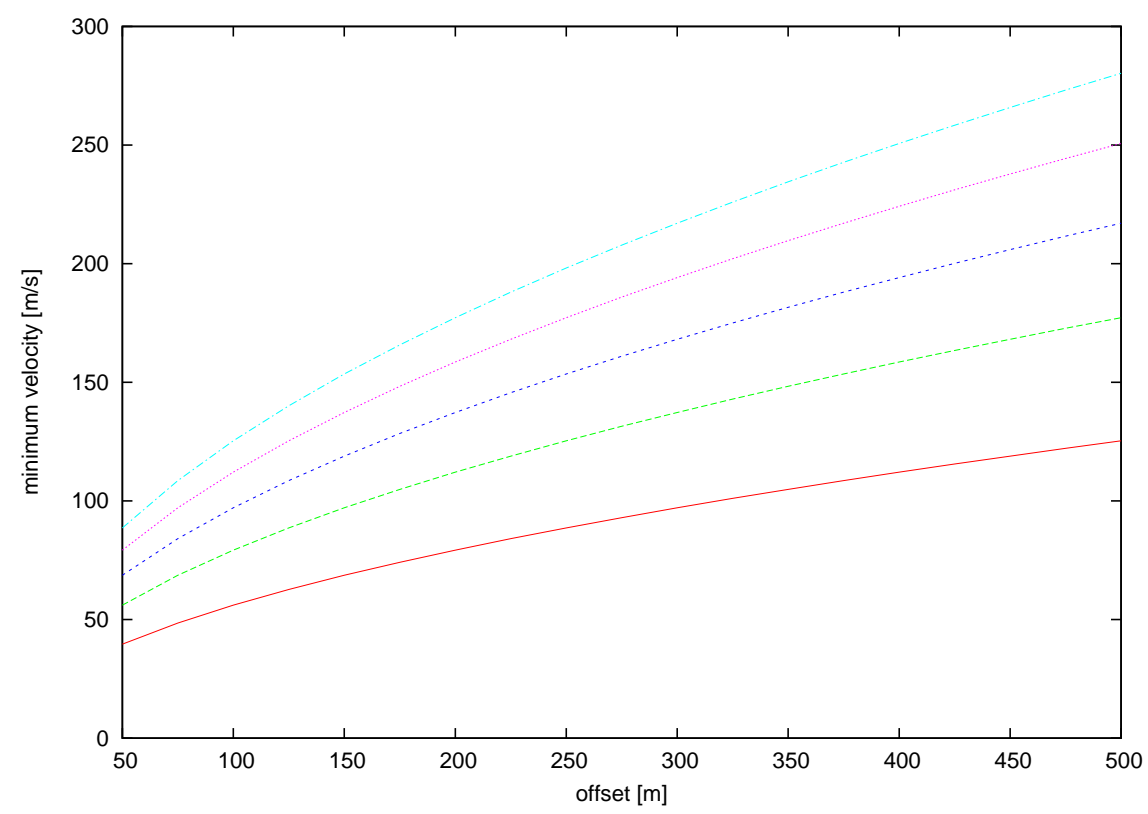

Figure 4.3: Lowest resolvable interface wave velocity in a stationary-receiver experiment with speeds from $0.5 \mathrm{~m} / \mathrm{s}$ (lowest line) to $2.5 \mathrm{~m} / \mathrm{s}$ ( $\sim 5 \mathrm{kn}$, top line) and $4 \mathrm{~Hz}$ sensors with respect to the source-receiver distance.

overcome, if the seismic shots could be fired at arbitrary absolute position, but usually only the relative position along a profile is obtained at sufficient accuracy. Hence, triggering the seismic source by positions controlled by an accurate navigation system connected to the source would enable to combine several profile runs to fill the gaps between individual runs. Since this navigation accuracy was not available, the acquisition configuration used for the experiments had to comply with the limitations encountered through the sampling theorem.

Using a single receiver and multiple shots, a common-receiver-gather of seismograms is obtained. Using the principle of reciprocity stating that one can interchange the position of source and receiver and still obtain the same travel times for both experiments, we can apply the wavefield transforms discussed in the previous chapter. This implies, that the seismic source used is repeatable, i.e. is comparable from shot to shot. If this was not the case, we would not expect to obtain a consistent phase relation between shots. Since coherent phase relations were obtained from the data examples presented in the second part of the thesis, it is assumed that the air gun sources fulfill the requirement of repeatability.

In consequence of the reciprocity theorem the variation of the phase relation is obtained at the source positions. This is in contrast to the situation, when multiple receivers are deployed at the sea floor. Then the phase relation is obtain for the variation between two adjacent re- 


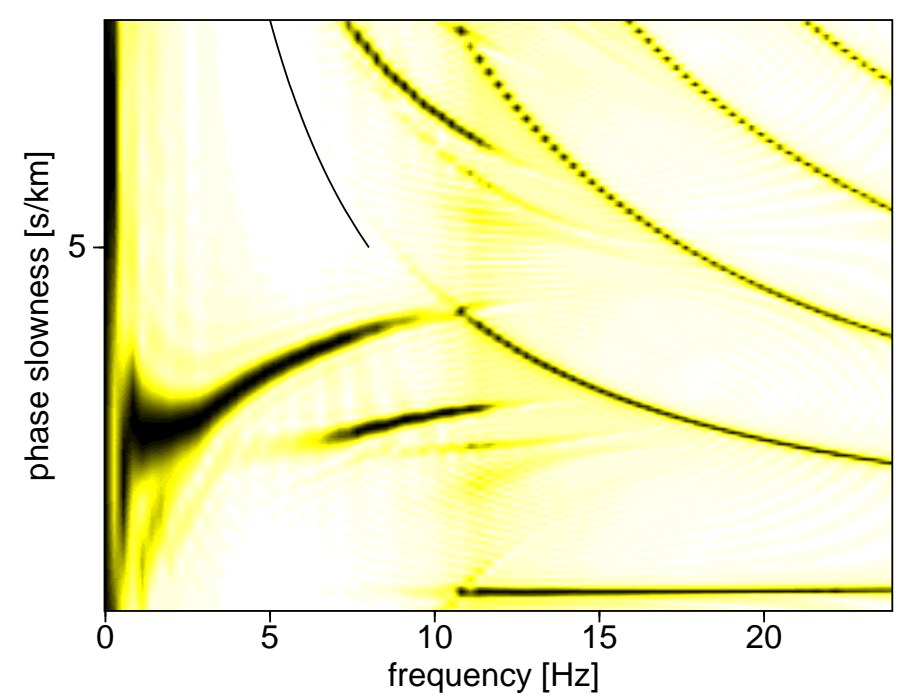

Figure 4.4: Data example to demonstrate the effect of aliasing. The amplitude of the dispersion curve is folded to reappear at zero slowness at the frequency where the hyperbolic line(black) meets the signal energy.

ceiver locations, where some disturbance might have occurred at deployment. Hence, using a common-receiver-gather, we obtain information of the subsurface properties in true undisturbed conditions.

\subsubsection{Common-Shot-Gather (OBC/Streamer)}

In order to overcome the restrictions encountered with stationary-receiver configurations a receiver array with a close receiver spacing to fulfill the spatial sampling criterion can be used. The receiver spacing of the system then determines the lowest resolvable shear wave velocity according to the relation in equation 3.1.

The length of the receiver spread determines the resolution in the slowness domain, if common receiver gathers of a single shots are used. This is the case if a towed receiver array in form of a streamer is used. The most dominant advantage of the towed system is the efficiency in terms of time required to cover a certain area of investigation. Secondly it implies to use a fixed acquisition geometry with respect to the research vessel, and therefore does not require an absolute positioning for data analysis methods. 
The deployment of an ocean bottom cable, independent of the sensor type to be used, resembles a combination of a stationary-receiver experiment with a fixed receiver geometry. While this still limits the range of operation, the limits in terms of the ship speed discussed for the stationary-receiver experiment no longer hold. If the length of the receiver spread is sufficient in terms of the requirements on resolution due to limiting the offset range, we do not need to consider limitations in progress due to spatial sampling. However, the restrictions on the minimum shot interval remain in order to record the full length of the slowly propagating dispersive interface wave. Using the combination of seismograms from different shots but the same receiver spread we obtain a mixed common-receiver and common-shot section with variation in offset. This can enhance the resolution, if the variation in the receiver coupling of different sensors and the variation of shot signals is either neglectable or accounted for. Furthermore, recording of the source signal with several receivers is essential as well as the recording of several shots with the same receiver (without changing the coupling) if the full phase and amplitude signals of the source and receivers is to be accounted for in the process of data analysis. 


\section{Chapter 5}

\section{Inferring Sediment Properties from Measured Dispersive Wavefield Spectra}

\section{Contents}

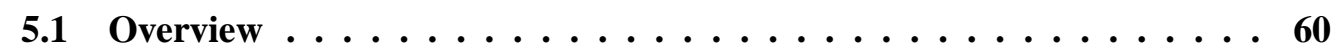

5.2 Modified Dispersion Curve Inversion $\ldots \ldots \ldots$. . . . . . . 61

5.3 Inversion of the full wavefield spectra $\ldots \ldots \ldots 6$

5.4 Adaption of the full wavefield inversion for marine seismic data $\ldots 68$

The main objective of the work presented in this thesis is to obtain information about the seismic velocity structure, with special interest on the shear wave velocity. Within this chapter I present and discuss the methods to obtain this information from the measured data. I suggest the application of an inversion of the full complex wave spectra recently developed for the Rayleigh wave inversion of land seismic data and show the advantages of this method compared to the alternatives. 


\subsection{Overview}

The aim of the investigation is to derive sub-bottom properties from measured data. The theory presented in chapter 2 indicated the complexity of the functional relation between seismic parameters and the phase velocity measured for various frequencies. Several approaches to simplify the problem have been used in the past which include the so-called wavelengthdepth method, the inversion of dispersion curves based on normal modes. Other approaches intended to use the complex relation with all the information and invert the full seismic waveforms.

The wavelength $(\lambda)$-depth method is the most simple and empirical approach sometimes applied in global seismology. For a given phase velocity-frequency pair observed in the data it is assumed that the representative depth $z$ of the measured velocity is a fraction of the wavelength $\lambda$. Gazetas (1982) recommended to use $\mathrm{z}=1 / 3 \lambda$ in general. While $1 / 2$ would be more appropriate for vertically homogeneous sites, a value of $1 / 4$ should be used at sites where the shear modulus increases significantly with depth (Gazetas, 1982; Matthews et al., 1996). This very simple method is not very satisfactory in terms of resolution and does not relate to the complexity of the influence of model parameters on the seismic wavefield.

In the previous chapters I have discussed methods to acquire dispersive seismic data and derive the spectral coefficients representing the seismic data. In this form the dispersive nature of the data can easily be visualized and analyzed. Further, I have presented methods to compute synthetic seismic wavefields from simplified models of the marine sediment. Having these methods at hand, we still require the solution to the inverse problem, hence to derive the sub-bottom parameters from the measured dispersive seismic wavefield.

The most challenging task with the respect of the interpretation of the measured seismograms is to try to predict each single wiggle of the measured seismogram by comparison with synthetically computed seismograms. The idea is probably best expressed by a citation of Wiechert, who stated that it seems an achievable goal to use and predict each single wiggle of a seismogram to determine the structure and composition of the crust of the earth ${ }^{1}$. The wave forms of synthetic seismograms are compared with the measured seismic data with respect to amplitude and phase which are determined by the viscoelastic model parameters. These methods are becoming more widely used, since the computational capacities have increased dramatically in the past few years (Nolet \& Dorman, 1996). However, it requires to have a very good idea of the model needed to describe the observed waveforms, due to the oscillatory nature of seismograms. Therefore not only computational problems have to be overcome with these methods, to be discussed at a later stage.

The aim is to simplify difficulties arising in the concept of full waveform inversion, but

\footnotetext{
${ }^{1}$ The original citation reads: "Es scheint ein erstrebenswertes und wohl erreichbares Ziel der experimentellen Seismik, jede Zacke, jede Welle der Seismogramme zu erklären und für die Entwirrung der Beschaffenheit der Erdrinde dienstbar zu machen." (Wiechert, 1926)
} 
still include the information of the amplitude and phase spectrum of the seismic wavefield. Methods to transform the measured seismic data into wavefield spectra and then compare these with synthetic spectra have been developed lately (Roth \& Hollinger, 1999; Forbriger, 2001). The inversion of dispersive waves can be combined for several dispersive wave types (e.g. Love and Rayleigh waves) (Krone, 1997), or other constraining information from the dataset, e.g. first arrival times (Forbriger, 2003b). This concept of jointly inverting independent information on the same properties is very useful to further constraint and stabilize the inversion. This enhances the resolution of the inversion result and facilitates the interpretation.

The inversion of dispersion curves is commonly applied to phase velocity dispersion curves obtained from the phase-difference method. The forward solution using normal mode algorithm requires to identify the mode number of the observed mode for the inversion process. Since the phase-difference requires an uni-modal wavefield, the mode identification for the inversion method seems not a further limitation at first sight. But, due to the complexity of the dispersive seismic wavefields observed in shallow targets, both on land and in the marine environment, it is a further limitation indeed. Even in data sets with just a single mode excited and observed this is not necessarily the fundamental mode. The mis-interpretation of an observed higher mode as the fundamental mode will lead to an inversion result which does not represent the sub-surface properties. This is one of the mayor disadvantage of the SASW methods and any other method based on mode identification and separation. For this reason it is intended to use an inversion method which does not require the identification of modes.

For the analysis of a multi-modal seismic wavefields it is suggested to investigate the seismic wavefield spectrum obtained from one of the wavefield transform methods described in section 3.3.1. Despite of the fact that the MFT method can be applied to multi-modal wavefields the resolution to distinguish individual modes is often not sufficient. The derivation of the seismic properties from the full measured wavefield spectra is now investigated with two different approaches, which have in common, that a mode identification is not required in either case. The first method is a variation of the commonly applied dispersion curve inversion. The second method is the inversion of the complex spectral coefficients discussed in section 5.3. The proposed methods seek to overcome the limitations of the dispersion curve inversion without inheriting the full complexity involved with the methods of fitting full waveform.

\subsection{Modified Dispersion Curve Inversion}

The concept of the modified dispersion curve inversion is to compare the observed dispersion curves only with those synthetically computed dispersion curves which would be excited with the source-receiver configuration used in the field experiment (Bohlen et al., 2003; Kugler, 
2001). The dispersion curves are picked from measured seismic data represented in the phase slowness-frequency domain. Extracting the information of the position of the dispersion relation with respect to frequency and slowness reduces the influence of noise and artifacts compared to using the full amplitude information of the wavefield spectrum. However, the use of only selected information may increases the ambiguity of the inversion which must be compensated by additional constraints. The dispersion curve picks are directly compared with maximum amplitude picks of the dispersion spectrum from the forward calculation using the propagator matrix method of Wang (1999). The model is parameterized in homogeneous layers and the inversion parameters include all shear wave velocities and layer thicknesses of the model. Linear positive gradients of the shear wave velocity variation with depth are allowed within selected layers. All other model parameters, i.e. the compressional wave velocities, densities and quality factors of the layers are fixed and must be obtained from a priori information or guesses. The inversion is performed with the sequential-quadraticprogramming algorithm (Grace, 1994).

The choice to invert for the shear wave velocity only is based on the observation that the Scholte wave dispersion is most sensitive to the shear wave velocity structure. The variations with density or compressional wave velocity are in most cases one or several orders of magnitude lower. It should be kept in mind, though, that this is not true in all cases. It is also possible that some special environments might be encountered where even the Scholte wave dispersion is affected by the influence of compressional wave velocity or density. This parameterizations is not suitable for the investigation of acoustic guided waves, for example. Those do require to account for compressional wave velocity variations since the sensitivity of the dispersion of acoustic guided waves to changes in th compressional wave velocity is high. However in most cases for the inversion of Scholte wave dispersion it is an appropriate method to reduce the number of free parameters to be determined, to those who are the most sensitive parameters. This helps to stabilize the inversion for the reduced amount of information yielded in the dispersion curves compared to full wavefield spectra.

\subsection{Inversion of the full wavefield spectra}

The inversion method used is a stabilized linear least squares inversion (Forbriger, 2003a; Forbriger, 2003b). I will start with an introduction of the general idea of the inversion procedures in order to discuss the problems and ambiguities which arise in application of the methods. I choose the linear least squares inversion method as an example, since this method is applied to the measured data presented in the second part of the thesis.

Generalizing the method to derive synthetic seismic wavefields as a forward problem, this is expressed by a functional operator $F$ which transfers the set of model parameters $m_{k}$ into the set of predicted properties. The search for the set of model parameters which describe the 
observed properties is the inverse problem and the method to obtain the solution of the problem is called inversion. This relates to a minimization problem since we seek to minimize the difference between the observed and predicted data. An error function $E$ is defined in order to quantify the difference between the predicted and observed properties which is formulated in terms of the quadratic norm in case of the linear least squares algorithm.

$$
E^{2}=|\vec{d}-F(\vec{m})|^{2}
$$

This difference or misfit of the predicted data is to be minimized and the criterion is obtained in form of the partial derivatives of the error function with respect to all free model parameters.

$$
\frac{\partial E^{2}}{\partial m_{k}} \stackrel{!}{=} 0 \quad \forall k
$$

I should note here, that in practice due to the presence of noise only a certain level of observed amplitudes can be explained by the model. Therefore a residual misfit should be tolerated to avoid the situation where the model parameters are fitted to artifacts or noise in the data which would lead to a mis-interpretation of the data. The choice of the tolerated misfit to separate useful data from unpredictable noise.

The partial derivatives of the error function are obtained from the derivatives of the functional relation $F$, which is assumed to be linear within a search range of all model parameters. If the relation $\mathrm{F}$ was truly linear, the required correction of the model parameters could be calculated directly from the misfit and the partial derivatives of $F$, apart from possible ambiguities. For the non-linear relation a certain range of parameter variations is defined. A linear approximation of the functional $F$ is used within this search range. Hence the search range corresponds to the range of validity of the linear approximation. The choice of search ranges of each parameters is another instrument to control the inversion process in terms of efficiency and accuracy in the process of determining the final model.

In most cases additional constraints must be used to stabilize the inversion process, which is definitely required if the number of model parameters $m_{k}$ is greater than the number of observables $d_{j}$ of the measured data.

$$
d_{j}=F_{j}(\vec{m})+n_{j}
$$

Due to the presence of noise $n_{j}$ in the measured data it is obvious that the model should not explain all the data, but some residues must remain. In the formulation of the noise contaminated data we assume that the unpredictable noise $n_{j}$ is statistically distributed with standard deviation $\sigma_{j}$ and average to zero. This enables to balance the individual observables $d_{j}$ by the standard deviation $\sigma_{j}$. Hence, the error function is defined by 


$$
E^{2}(\vec{m})=\sum_{j=1}^{M} \frac{\left|d_{j}-F_{j}(\vec{m})\right|^{2}}{\sigma_{j}}+\sum_{l}^{L} S_{l}(\vec{m})
$$

The first term will be denoted the so-called misfit function $\chi^{2}$ representing the misfit between the measured and predicted data. The second term includes the stabilizing constraints $S_{l}$ which generally depend on the model parameters. The choice of constraints depends on the a priori information included. The correlation between two model parameters or restrictions to a certain range of validity of a parameter are just examples of a variety of possible constraints. The choice of constraints is a third instrument to control and influence the final results of the inversion. The formulation in equation 5.2 treats all data values $d_{j}$ with equal weight, but this is not favorable e.g. if the data values have different physical meaning. Individual weighting between the observables as well as for the stabilizing terms enables to treat several independent data sets in the same inversion procedure with respect to additional constraints. This is achieved by introducing the diagonal matrix $W$ composed of the individual weights $w_{j}$ with recipicle unit to the data value $d_{j}$. Thus the misfit function reads

$$
\chi^{2}=|W(\vec{d}-\vec{F}(\vec{m}))|^{2} \quad W=\operatorname{diag}\left(w_{j}\right) .
$$

The transition to the weighting with the $w$ instead of $\sigma$ is just a generalization, since for $w_{j}=1 / \sigma_{j}$ the error function will be identical to eqn. 5.2. The optimized model parameters are obtained by applying the corrections to each model parameter which is calculated from the solution of the system of linear equations obtained from the minimization condition 5.1. The calculation of the correction values involves the solution of a system of linear equations containing the misfit of all input data as well as all the constraints (Forbriger, 2001; Parker, 1994, for details).

In case of a linear function $F$ a single set of correction values would lead to the optimized model. In practice the functional relation $F$ is non-linear, but the function is assumed to be linear within a certain search range from the current model and the inversion procedure is iteratively continued for the search ranges of the linear approximation of the functional until the misfit is reduced to an acceptable value. In summary we have identified four mayor instruments to control the inversion procedures:

1. model parameterization

2. tolerated misfit

3. stabilizing constraints

4. weighting functions 
The influence of these parameters will be discussed in the following.

Generally the first arrival times are picked in the seismograms to be used for the joint inversion of the first arrival times and the spectral coefficients. The refraction analysis of the arrival time picks can be used for the definition of the initial model. From the transformed wave spectrum the careful selection of the frequency-slowness range to be used for the inversion can eliminate parts which are contaminated with noise. This can help to stabilize the inversion. However, noise free portions of the spectrum without significant amplitudes can be useful data input, since the absence of modal energy may be just as valuable information as the presence of seismic energy. Hence, the amplitude level of noise must be determined since this is used to determine the level of remaining residues in the error function which should not be inverted.

The spectrum can also be used to infer initial guesses for the starting model. Asymptotic velocities for the low and high frequencies as well as parts of little change in velocity for a frequency range are often associated with shear or compressional velocities of a certain layer. Also, the number of modes within a certain frequency range is determined by the layer thickness of guiding interfaces. Together with additional a priori information on the stratigraphy this information must be used to define the parameterization of the subsurface model in terms of layers with constant parameters or linear or second order polynomial changes. The number of layers as well as the polynomial order of the changes in seismic velocities, density and quality factors must be chosen in good compromise to limit the number of parameters of the model to a minimum, but on the other hand, allow for sufficient parameter variations to obtain a model to adequately explain the measured seismic wavefield.

Hence, before starting the inversion we require

- the spectral coefficients of the seismic wave field from a seismogram section with adequate spatial and temporal discrdiscretisationitisation and appropriate editing, muting and tapering to avoid artifacts during the wavefield transform.

- an appropriate start model of the subsurface to generally represent the dispersion pattern found in the data. The information can be inferred from seismic interpretation as well as a priori knowledge from the study area, e.g. rough assumptions of the geological setting.

The error function $E$ is minimized according to equation 5.1 and includes the weighted misfit between the measured and observed data as well as four stabilization terms.

$$
E^{2}=\left|W\left(\vec{d}-\vec{F}_{0}-D \delta \vec{m}\right)\right|^{2}+\sum_{l} S_{l}^{2}
$$

with 


$$
\vec{F}=\vec{F}_{o}-D \delta \vec{m}, \quad \vec{F}_{o}=\vec{F}\left(\vec{m}_{o}\right), \quad \delta \vec{m}=\vec{m}-\vec{m}_{o} \quad D_{j k}=\frac{\partial F_{j}(\vec{m})}{\partial m_{k}}
$$

Here, $\vec{F}_{0}\left(\vec{m}_{0}\right)$ denotes the predicted spectral coefficients computed from the initial model $\vec{m}_{0}, D \delta \vec{m}$ are the partial derivatives of the parameter variations. The first term corresponds to the weighted misfit function $\chi^{2}$ with diagonal matrix $W$ composed of the parameter weights $w_{j}$ as mentioned above.

The stabilizing terms $S_{l}$ relate to constraints from linearization and smoothing as well as other constraints if applicable. Recalling the complexity of the processes involved in the propagation of the stress field within a complex porous medium, it is clear that the number of unknown parameters is much greater in reality than the observables and therefore the solution of the inversion problem is undetermined. Constraining the search for a model to the most simple model to adequately describe the measured properties enables to obtain a solution to the problem at all. Parker (Parker, 1994) formulated the inversion in the form of this elementary a-priori requirement and interpreted the misfit of the data as a constraint to the inversion for a smooth model description. Hence he formulated a Lagrange-functional

$$
L(\nu \vec{m})=\|S \vec{m}\|^{2}+\nu\left(\chi^{2}-M^{2}\right)
$$

where $\chi^{2}$ denotes the misfit function in terms of the statistical variance of the measurements $\sigma_{j}$ as formulated in equation 5.2. The stationarity requirement for $L$ in terms of the Lagrange parameter $\nu$ and the model parameters $m_{k}$ is $\frac{\partial}{\partial m_{k}}\|S \vec{m}\|^{2} \stackrel{!}{=} 0$ and leads to the best model to simultaneously fulfill the a-priori requirement in agreement with the measured data in the order of the significance with respect to measurement uncertainties. A simplified method of this concept is used for the least squares inversion and the modification reads to

$$
E^{2}=\nu\|S \vec{m}\|^{2}+\chi^{2}
$$

and the parameter $\nu$ is used to determine the weighting of the constraint. The applied constraint formulates that variations of model parameters are to be small compared to the search range $r_{k}$.

$$
S_{1}=\left|C_{1} \delta \vec{m}\right| \quad C_{1}=\operatorname{diag}\left(\frac{1}{r_{k}}\right)
$$

This stabilizes the inversion and restricts the variations to be within the range of linearization. The search ranges $r_{k}$ have to be chosen accordingly for each model parameter.

A constraint in form of a smoothing function in $\mathrm{z}$ could be useful in order to keep the model to be smooth and as simple as possible. This is an important constraint since there will always be a more complex model to fit the data as good or better than a simple model. Yet, the information of the measured data is restricted and we therefore seek for the most simple 
model which adequately represents the measured data to a level which is not exceeding the accuracy of the measurement or the noise level of the data. This concept is also relevant for the choice of the model parameterization. Generally this would suggest to use the most simple parameterization possible to explain the observed properties of the seismic wavefield. However, since in practice the model parameterization is not changed in the inversion process, it is favorable to select a model parameterization as well as the free inversion parameters generously to enable more complex model structures in the process of the inversion if the data requires them. The constraint on smoothness will prevent the inversion algorithm to choose complex models if not driven and therefore required by the measured data. The number of model parameters and specially the number of free inversion parameters is therefore mere a compromise in terms of the memory and computational capabilities of the computer rather than a conceptual limitation.

In practice this requires to limit the number of free parameters. By experience we know, that variations in density and compressional wave velocity have low impact on the dispersion of Scholte-waves, while their dispersion characteristics is highly sensitive to variations in shear wave velocity. Therefore it is sensible to put constraints to density and compressional wave velocity and invert for shear wave velocity in Scholte-wave data. However it is not always true that Scholte-waves are insensitive to compressional wave velocity and density and it should be checked e.g. by investigating the partial derivatives for these parameters. For the inversion of guided waves the compressional wave velocity is a key parameter and should be used as a free inversion parameter.

In general the choice of seismic velocities and layer thicknesses as simultaneous free parameters should be treated with care since these are likely to be trade-off parameters and the inversion can tend to just oscillate between two equally good models. The presence of a trade-off is obvious in the case of travel time inversion with respect to layer thickness and velocity. A specific travel time arrival can be obtained equally well by adjusting either the velocity within the layer or the length of the propagation path in terms of the layer thickness. Either of the cases and any combination of both parameters to fulfill the travel time criterion are numerical solutions to the inverse problem. Hence, an infinite number of models will explain the observed travel time equally well and thus I denote these parameters as a pair of trade-off parameters.

I have discussed the numerous parameters which control the inversion process which I summarize with respect to their application prior or within the inversion scheme in table 5.1.

The inversion procedure is performed interactively by controlling the weights of the constraints and free parameters, adjusting the balance between the simultaneous inversion of first arrival times and spectral coefficients and checking for plausibility of the current inversion result. Since an optimized model may present a local optimum rather than the global minimum 


\begin{tabular}{|c|c|c|}
\hline control mechanism & parameter & Number \\
\hline \hline weighting of greens coefficients & $w_{j} \forall$ data coefficients & $\approx 120000^{1)}$ \\
\hline tolerated noise level & $t t_{\text {err }}$ and $g_{\text {err }}$ & 2 \\
\hline model parameterization & $\begin{array}{c}n \text { layers with } 5 \text { properties } \\
\text { of polynomial order } p_{k l} \leq 3\end{array}$ & $\begin{array}{c}\text { usually } 10-30^{2)} \\
\text { from } \sum_{l}^{n} \sum_{k}^{5} \sum_{j}^{p} m_{j k l}+n\end{array}$ \\
\hline balancing factor & $\chi^{2}=b \chi_{\text {greens }}^{2}+(1-b) \chi_{t t}^{2}$ & 1 \\
\hline stabiliation parameter & $\nu$ & 1 \\
\hline search ranges & $r_{k}$ & $0-30$ \\
\hline source spectrum & $s_{j} \forall$ data coefficients & $1^{3)}$ \\
\hline
\end{tabular}

1) The number of spectral coefficients depends on the the sampling ( e.g. 300x400).

2) The number of model parameters is a selection from $\sum_{l}^{n} \sum_{k}^{5} \sum_{j}^{p} m_{j k l}+\mathrm{n}$ layer thicknesses.

3) The choice between inverting the source spectrum in order to obtain full amplitudes and the application of a normalizing term is the free parameter.

Table 5.1: Control parameters for the inversion of full wave spectra. The error levels for the travel times and greens coefficients are denoted $t t_{\text {err }}$ and $g_{\text {err }}$ and correspondingly the misfit functions $\chi_{t t}^{2}$ and $\chi_{\text {greens }}^{2}$ for the data sub sets of the travel time and greens coefficients.

of the optimization problem, usually several manual interactions are required to continue the inversion prior to the acceptance of the final model.

A resolution analysis with the final model shows the trade-off between parameter fits of several free parameters to partially compensate for a given variation in layer thickness. The resolution analysis is performed as a "rubber band test" by a sequence of inversion runs. Each of the free inversion parameters is fixed at levels 10\% higher and lower than in the final model, while the other free parameters are inverted by consecutive runs. The set of models produced within the sequence of all inversion runs define the range of variations of the free parameters, and for each free parameter trade-off parameters can be identified. Despite of the importance of a resolution analysis for the interpretation of the inversion result I refrain from a more detailed description but refer to Forbriger (Forbriger, 2001).

\subsection{Adaption of the full wavefield inversion for marine seis- mic data}

The original inversion described by Forbriger assumes a stack of solid layers and uses a forward algorithm based on the reflectivity method (Forbriger, 2001). In order to calculate the Green's coefficients for models with a liquid layer on top, we need to account for the 
modified boundary conditions at the liquid-solid interface at the sea bed. This was achieved by implementing the forward algorithm of MSEIS based on the propagator matrix method, which already included the implementation of boundary conditions for liquid layers (Wang, 1999). This algorithm also produces the spectral coefficients for a layered medium in the slowness-frequency domain efficiently and is therefore suitable for the inversion scheme.

At intermediate stages additional problems were encountered in forward calculations of models with source and receiver in the water column at the same depth, or little difference in depth. In these cases large amplitudes at low frequencies were observed. The crossection
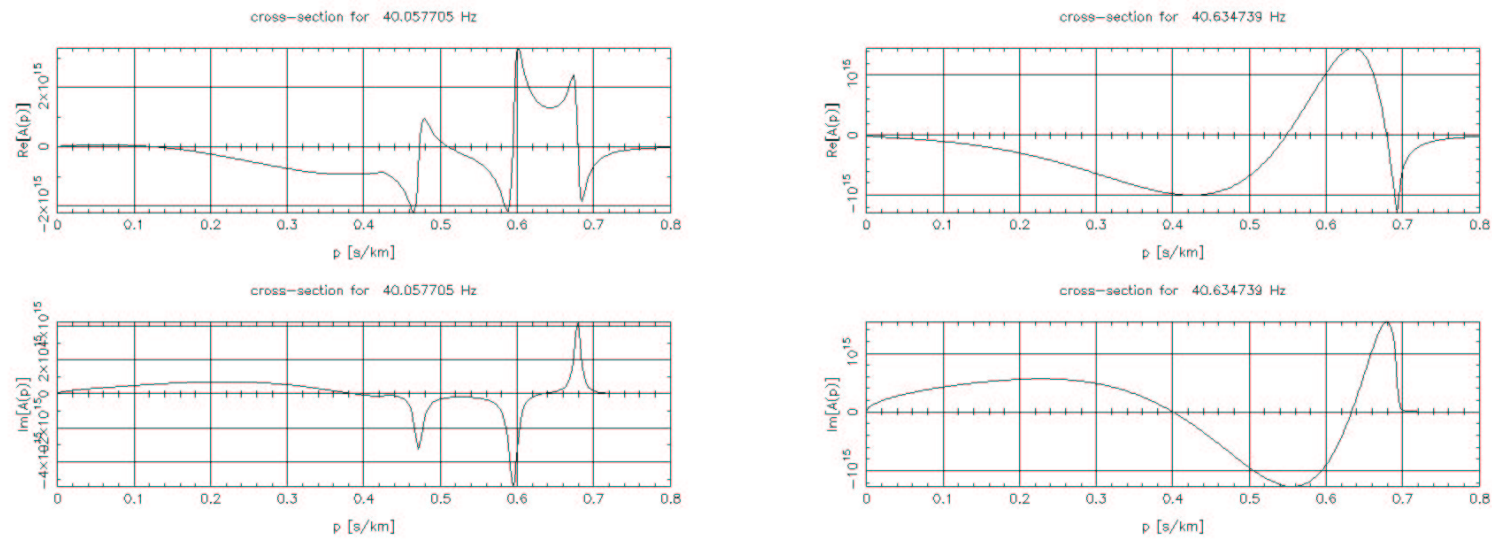

Figure 5.1: Crossection of the uncorrected (left) synthetic wavefield spectrum at frequency $40 \mathrm{~Hz}$ and the correction term to be applied (right). The real (top) and imaginary (bottom) part of the complex spectral coefficients is plotted with respect to phase slowness at constant frequency. The peaks relate to the fundamental and higher modes of the seismic wavefield. In the uncorrected spectrum a large negative contribution shifted the location of the modes with respect of slowness corresponding to the spectral coefficients of the correction term.

in fig. 5.1 shows that the mean of the amplitude (real) signal is not zero but a negative contribution to the integral is observed. This contribution arises from the correction term shown in the lower image. This was interpreted as contributions of the near-field term in the forward calculations. Since this only contributes at very small offsets, it is not relevant for the calculation of synthetic seismograms and is not seen in the measured data. Therefore this "near-field correction" was implemented in the inversion algorithm and applied to the synthetically calculated spectral coefficients. The comparison of the corssections (Fig.) and the spectra (Fig.) for a measured data set and the corrected synthetic spectral coefficients shows both the requirement and the successful application of the correction. This near-field 

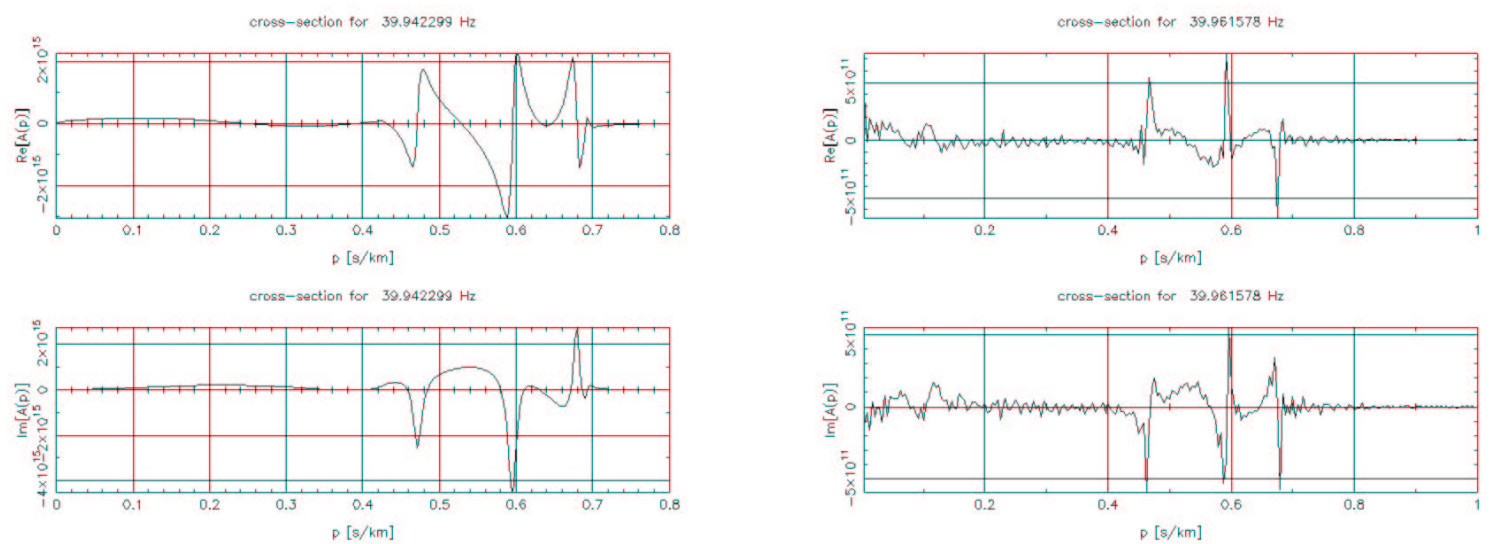

Figure 5.2: Crossections at $40 \mathrm{~Hz}$ for the corrected synthetics and measured spectral coefficients in the representation corresponding to fig. 5.1.

correction is applied in the inversion by calculating the Green's coefficients for a water half space with the given source/receiver depth and subtracting these from the forward solution of the layered model. The correction coefficients therefore have to be recalculated only, if the seismic parameters of the water column or the assumed source and receiver depth are changed, but remain constant otherwise, which reduces the number of additional forward calculations in the inversion process. The implementation of the majority of these adaptions into the code was done by Thomas Forbriger and his help is gratefully acknowledged.

The investigated methods consider the full wave energy excited by the seismic source and propagated through the medium. However, the amplitudes of seismic data are not only influenced by variations of the medium, but to great extends also depend on the variation of factors related to the source and receiver, namely source repeatability ${ }^{2}$ and variations in receiver coupling. Furthermore, the variations in the path of propagation, not alone determined by variations in the source-receiver geometry, affect the measured seismic wavefield. This has to be accounted for in both of the latter groups of methods and their difference is predominantly determined in the choice of data space. Seismic waveforms are non-linear oscillatory signals, not a simple task for an inversion algorithm, since the oscillation is projected into the misfit function to be minimized during the inversion process. It is therefore advantageous to use wavefield spectra. The Green's coefficients have been determined in application of a wavefield transform, which separates the oscillatory part of the wavefield in the transform process (Forbriger, 2003a). Hence, linear inversion of the Green's coefficients of the wavefield spectra is much more stable compared to the inversion of seismic waveforms.

\footnotetext{
${ }^{2}$ source strength and amplitudes and phase of the source signal, Verbeek and McGee, 1995
} 


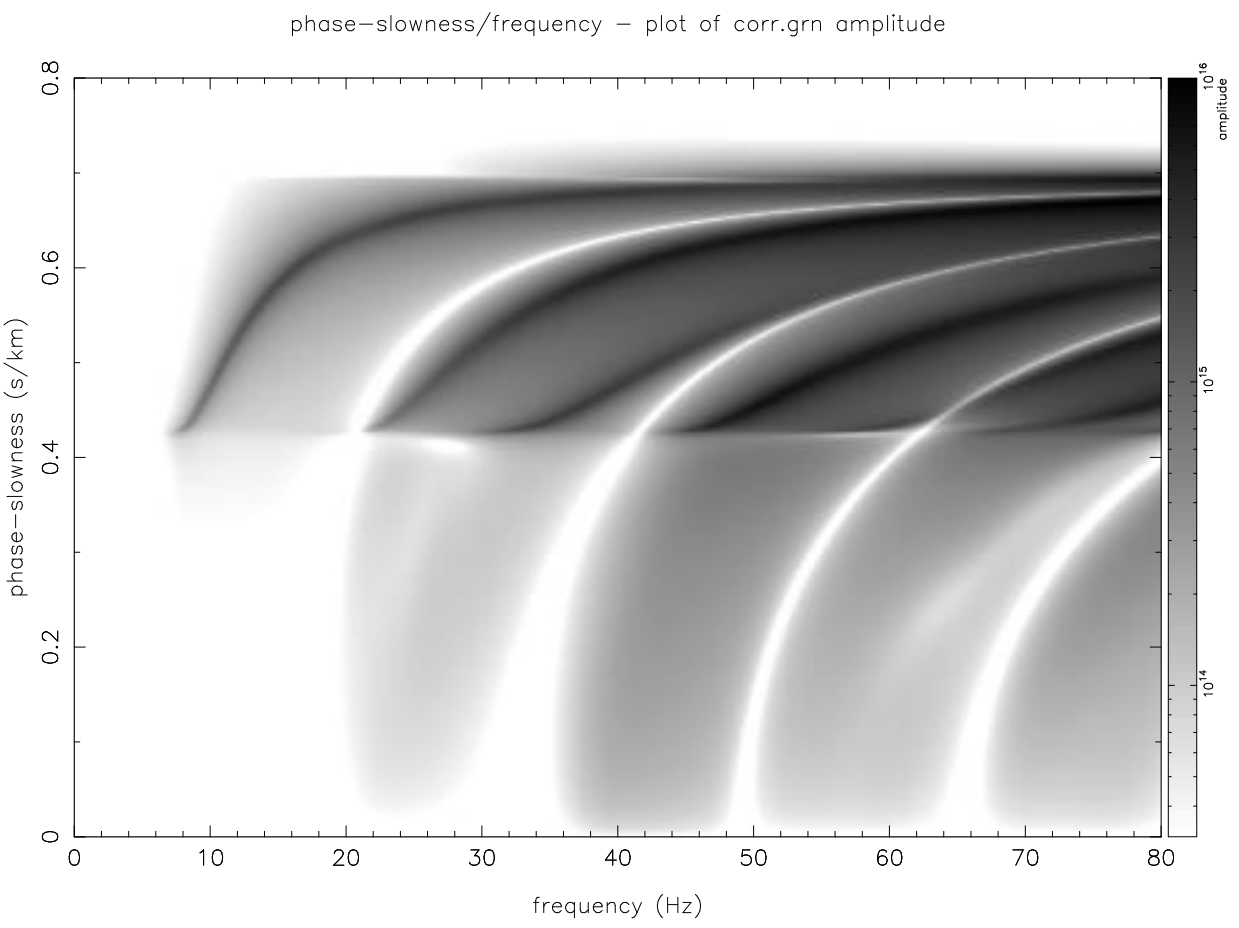

phase-slowness/frequency - plot of syn.grn amplitude

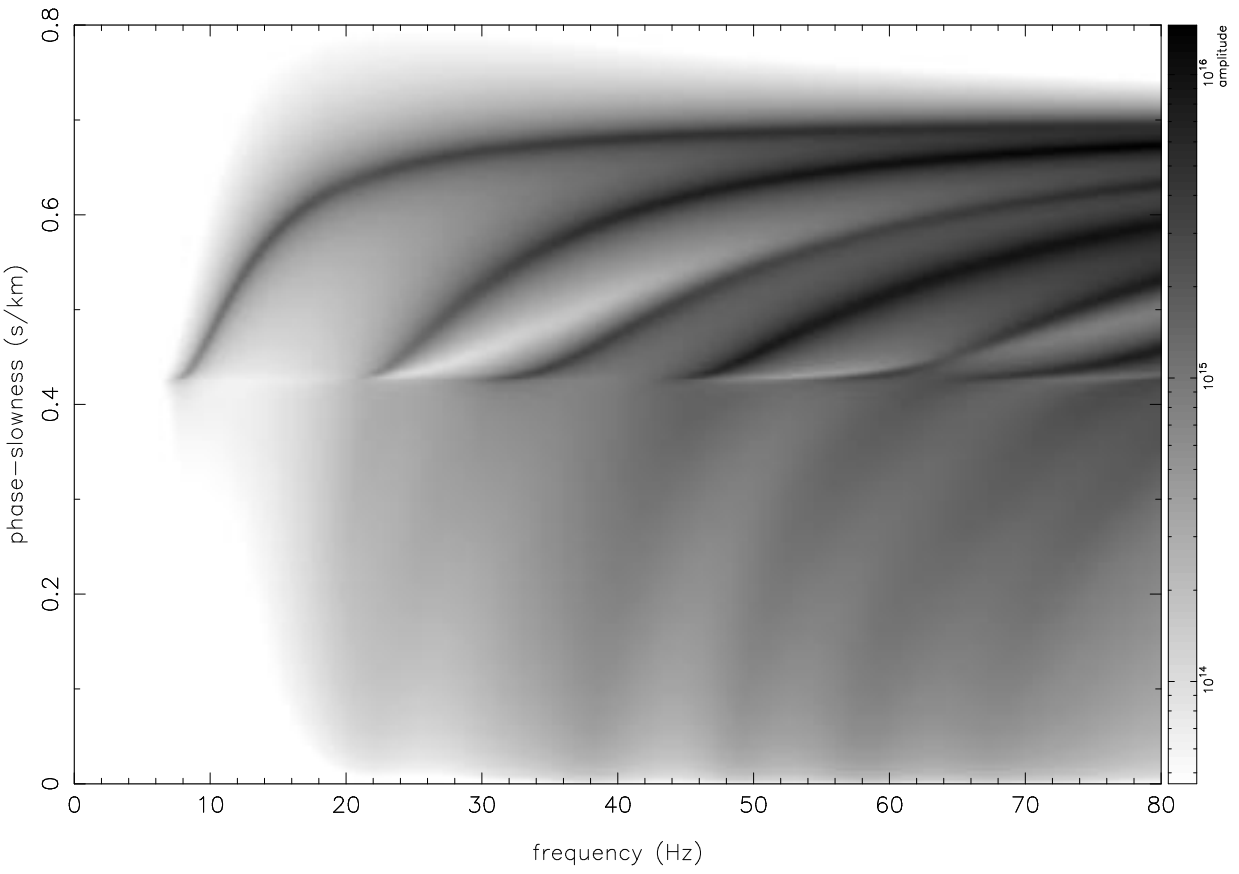

Figure 5.3: Synthetic wavefield spectra with and without the near-field correction applied. 


\section{Part II}

Case studies 



\section{Case Studies}

The improvement of the acquisition and analysis methods has been continuously developed during my work on three mayor projects with data sets from the Kiel Bay and Arkona Basin of the Baltic Sea and the Laptev Sea in North Siberia. The multi-channel streamer seismic data from the latter has been provided by the Federal Institute of Geosciences and Resources (BGR). It was used to develop analysis methods and further the understanding of dispersive guided waves. The study areas in the Baltic Sea have been used to test and further develop the acquisition of dispersive seismic data. The inversion procedures to infer in-situ seismic properties from the dispersive seismic wave spectra has been continously enhanced and used for all data sets. Scholte wave measurements in the Kiel Bay area have been conducted to investigate the potential to excite and record Scholte waves with surface towed airgun and ocean bottom sensor stations. Together with first analysis methods this is the topic of the SCHERSEIS project in the first case study (Chapter 6).

Further developing the acquisition towards a towable system as well as the correlation of the inversion results with sedimentological data was the scope of the investigation in the third case study from the Arkona Basin, which has been devoted as a Natural Laboratory (NATLAB) in the subproject IngGAS-NATLAB. Here, recordings of Scholte waves as well as acoustic guided waves are discussed (Chapter 8). But before investigating the detailed analysis in the Arkona Basin, I present a data set from the Laptev Sea, North Siberia, as an introduction to the potential of propagating leaky modes in form of guided waves in the second case study in chapter 7. This data set from the Laptev Sea and the investigation on dispersive waves were the trigger for the ongoing investigations on the potential of developing a towable acquisition system to determine sediment properties from the measurement of dispersive wave types.

The location of the study areas is marked on the bathymetric maps of the Baltic Sea and of the East Siberian Sea (Fig. 6.1). 

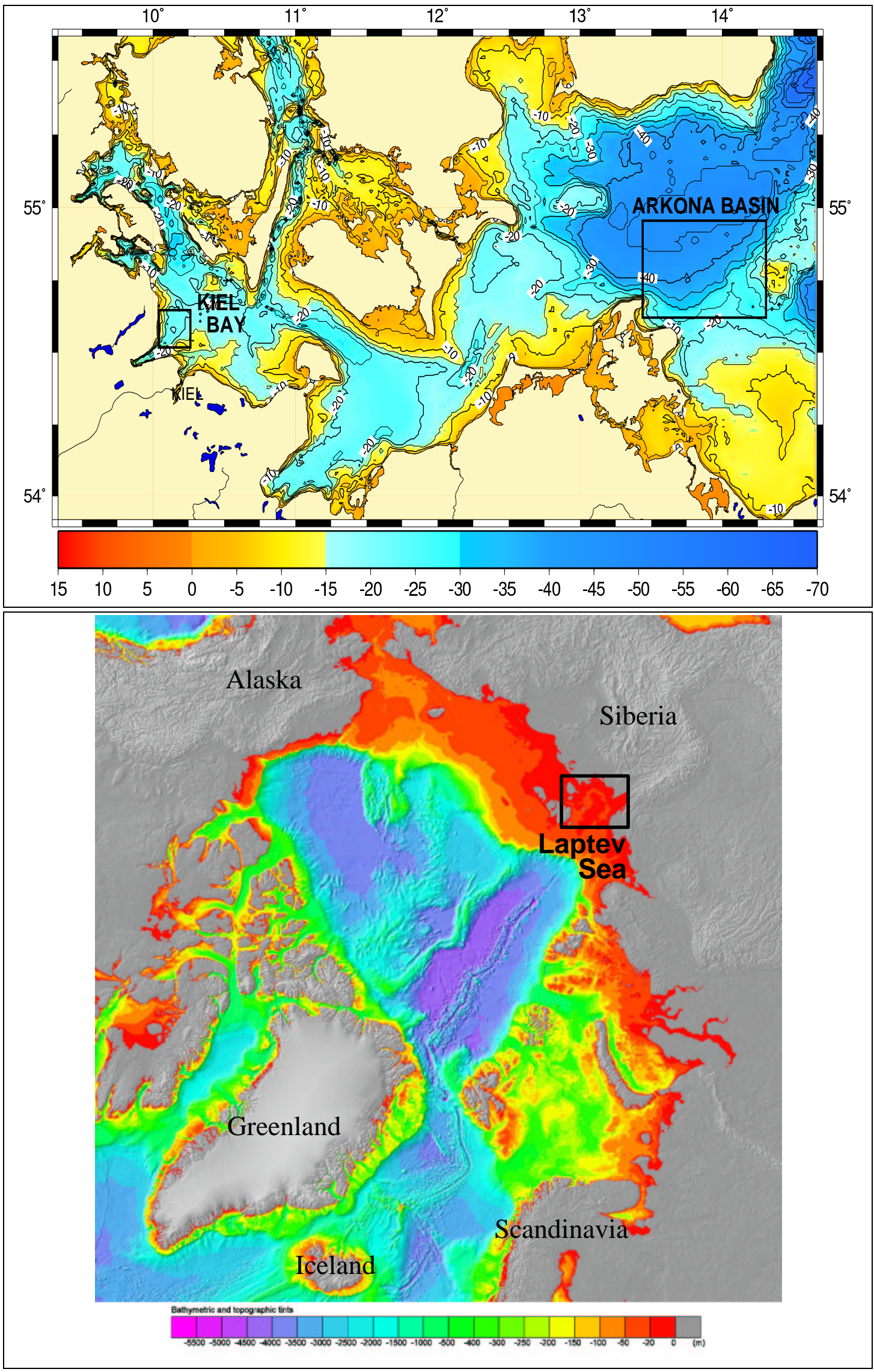


\section{Chapter 6}

\section{Scholte Waves - Acquisition and Processing - Kiel Bay}

\section{Contents}

6.1 Introduction $\ldots \ldots \ldots \ldots \ldots \ldots \ldots \ldots \ldots$

6.2 Seismic experiments . . . . . . . . . . . . . 78

6.2.1 Time synchronization of the source and receiver systems . . . . . 82

6.2.2 Determining and achieving the required shot interval . . . . . . 83

6.3 Data examples, acquisition and preprocessing . . . . . . . . . 83

6.3.1 Comparison of different receiver systems . . . . . . . . . . . . . 84

6.3.2 Scholte wave dispersion at different geological sites . . . . . . . 91

6.4 Conclusions . . . . . . . . . . . . . . . . . 94

Figure 6.1: (left page) The bathymetry map of the Baltic Sea shows the locations of the study areas of the Kiel Bay and Arkona Basin case studies presented in chapters 6 and 8 (top). The Laptev Sea study area described in chapter 7 is indicated in the bathymetric map of the Polar Seas (bottom, courtesy of IOBAC, 2003). 


\subsection{Introduction}

The aim to directly measure shear wave velocities of shallow marine environments lead to the marine seismic experiment conducted in the Baltic Sea in 1987 using a sledge-mounted seismic source operating at the sea floor in conjunction with an ocean bottom receiver spread (Gimpel, 1987). A high amplitude Scholte wave has been recorded, but, once operating at the sea floor, direct measurements of the shear wave velocity were more easily obtained.

The potential to derive shear wave velocities from the Scholte wave has become of interest since it was realized, that these waves do propagate measurable seismic energy into the water column, while pure shear wave energy is not observable. Probably one of the first multi-channel streamer records of a Scholte wave was acquired in the Laptev Sea in 1997, by the Federal Institute of Geosciences and Resources (BGR, Hannover) in collaboration with the Sevmorneftegeofizika (SMFG, Murmansk) (see chapter 7). Since the Scholte wave was only recorded on very selected parts of one of the seismic lines, we initiated an investigation to determine how to excite and record these Scholte waves in form of the DFG funded research project SCHERSEIS. Here, the scope of the investigation was to use an ocean bottom sensor in order to investigate, that a surface towed airgun can excite sufficient Scholte wave energy to derive shear wave velocity information from the measured dispersive seismic data. In the scope of this project we conducted two marine seismic experiments in the Kiel Bay study area on FK Litorina and FK Polarfuchs in 1999 and 2000. In the following, I will present the investigations concerning the acquisition and data inversion methods developed in cooperation with the project team of SCHERSEIS. In the first experiment, three different receiver types and configurations have been used to investigate the acquisition system and parameters to enable Scholte wave measurements. The second experiment in 2000 was conducted at three different geological sites in order to investigate the potential to distinguish the subsurface material from the measurements. The analysis methods, firstly to depict the dispersion characteristics of the seismic wave field and, secondly, to derive the shear wave velocity from the measured dispersion, have been developed.

\subsection{Seismic experiments}

In September 1999, the first seismic experiment was conducted with three different receiver systems in order to investigate the influence of receiver coupling and the repeatability of the airgun signal. Three ocean bottom sensor systems had been deployed simultaneously, during the experiment at site A of the Kiel Bay study area. Next to the autonomous Ocean Bottom Hydrophone (OBH) station from GEOMAR (Kiel) and an Ocean Bottom Seismometer (OBS) station from GeoPro (Hamburg), both deployed from FK Litorina, a 48-channel ocean bottom hydrophone array from Electronic Engineering Ltd., UK, was deployed from FK Po- 
larfuchs, which then ankered during the ship-board registration of the seismic data using the Geometrics Strata View R60 seismic recorder 6.2. Seismic sources were several Praklaairguns of the type VLF and VLA with chamber volumes from 0.3 to 1.2 were used at various operating pressures ranging from 60 to 120 bars, operated from the research vessel FK Litorina. The location of the study area for this experiment, which coincides with the location of the seismic experiments conducted the following year, is presented in fig. 6.3. The two sites $\mathrm{A}$ and $\mathrm{B}$, with water depth less than $25 \mathrm{~m}$, have been of special interest in previous studies. The outcrop of glacial till at the sea bottom at site A was recognized in previous seismic investigations and was therefore chosen as an example of hard sea floor sediments in contrast to the several meter thick layers of soft Holocene mud filling the troughs of glacial or fluvial discharge channels present at site B. The occurrence of scattering effects as well as the structure of glacial or fluvial channels have been investigated in a 3-D experiment, hence detailed knowledge of the subsurface structure is available at this site (Müller et al., 1999).

During the seismic experiments in August 2000, next to measurements at the sites A and $\mathrm{B}$, the site $\mathrm{C}$ was investigated in order to enhance the variety of different sub-bottom materials. Grab samples from the area indicated a sand or sandy-silt with cobbles and rocks as well as shells and organic material. From the results of the previous cruise, the choice of acquisition system was made in favor to multiple OBS stations to be used in a walk-away configuration. Five OBS stations were available for the experiment and deployed in line and crossed with an 0.61 airgun source in two sets of perpendicular profiles at 45 degree angle, forming a star configuration. The main profile crossing the receiver stations in in-line

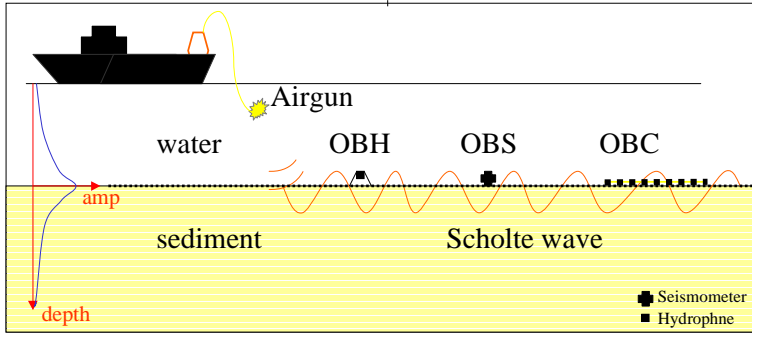

(a) Configuration of the experiment on cruise Li1'99, September 1999

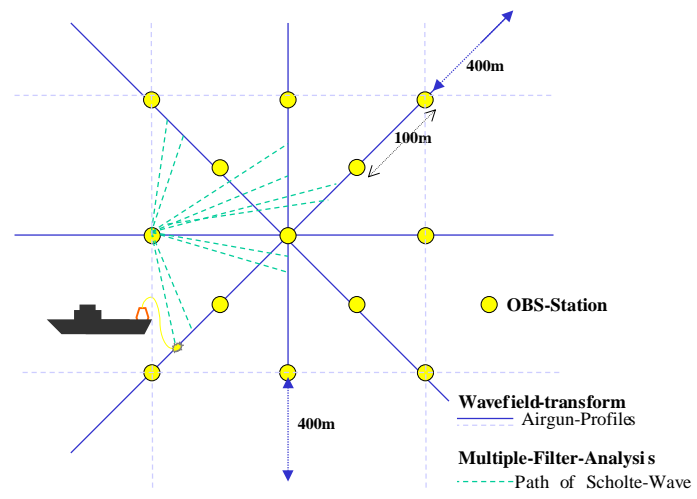

(b) Experiment layout for Aug 2000 Cruise Li-2’00

Figure 6.2: Survey configuration in the first seismic experiment at Kiel Bay, Baltic Sea, Sept. 1999 


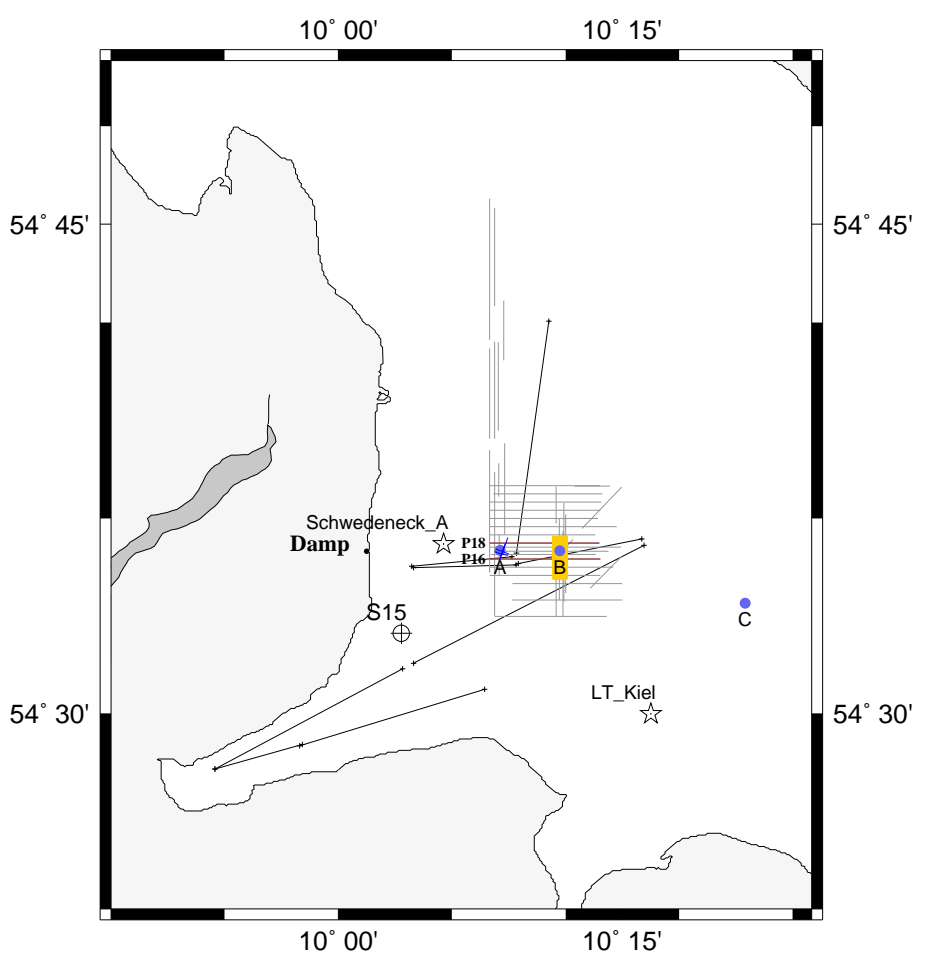

Legend
- Study area Littorina/Polarfuchs 2000
Previous Cruises

+ ALKOR 103/96: Boomer (SC) + EMG4

ALKOR 126/98: Boomer (MC) AWI-Streamer

ALKOR 99: Boomer (MC) AWI+IFG Streamer

- Littorina 99: Airgun + OBC + OBH + OBS

SC/MC=Single/Multi Channel; H=Hydrophone; S=Seismometer

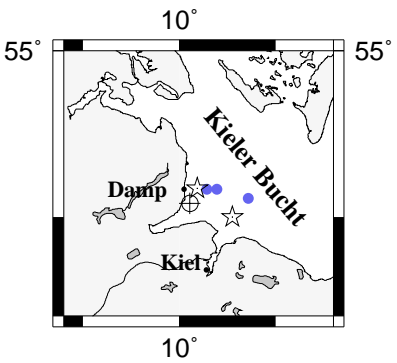

Figure 6.3: Location of the two surveys in the Kiel Bay, Baltic Sea. The first Survey (1999) focused on site A, while measurements at A, B and C were obtained in 2000. 
direction was extended to double the profile length. Then, all except the center station were moved to form a perpendicular receiver line in relation to the first and the star configuration profiles were repeated. It was possible to accomplish one survey on each measuring day with six hours of time available in the study area. The survey was conducted at the three different sites $\mathrm{A}, \mathrm{B}$ and $\mathrm{C}$ and a second survey at 45 degree angle was added at site B. Additional highresolution seismic lines were acquired with a boomer source and single channel registration. The location of all profiles is shown in fig. 6.4.

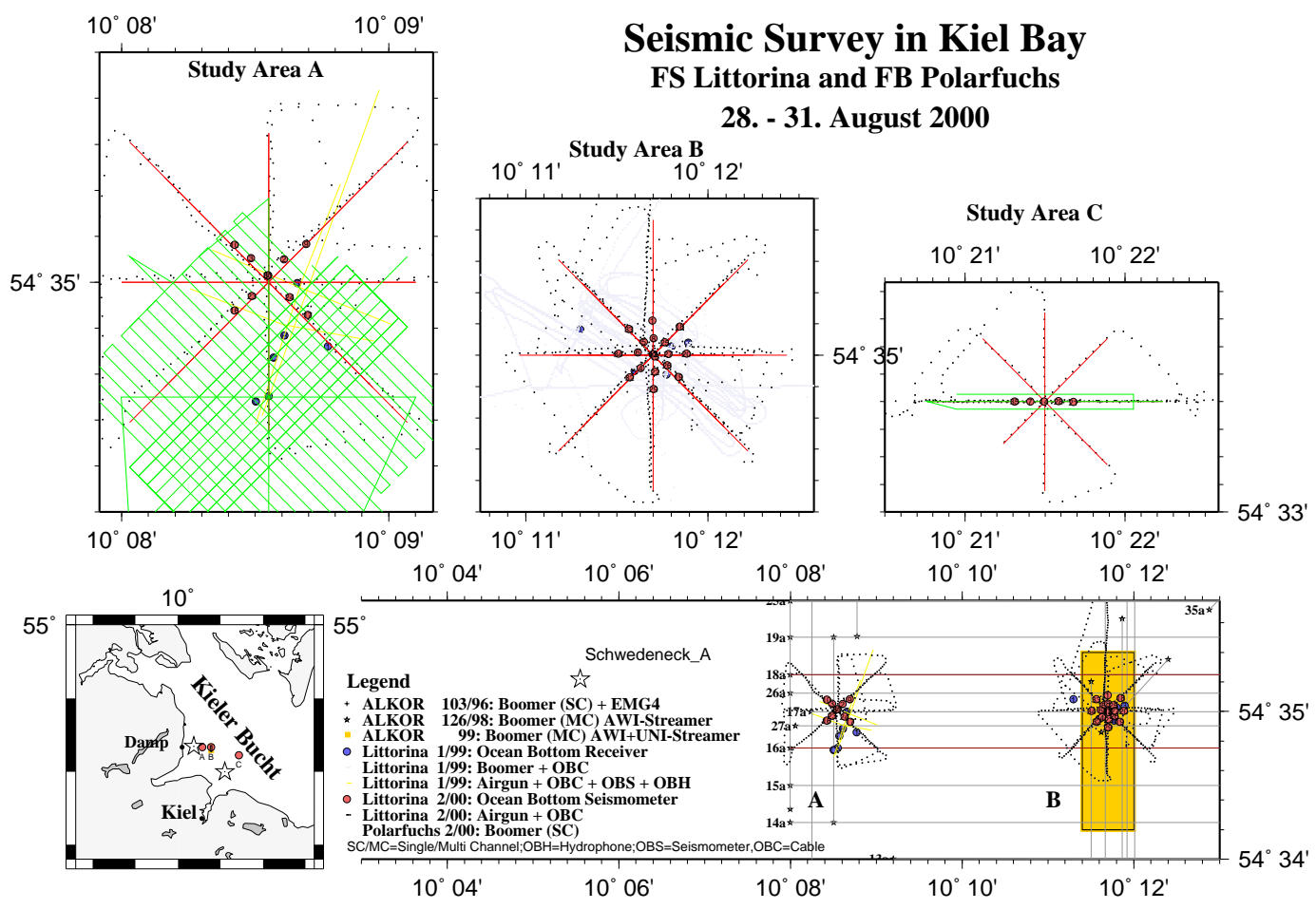

Figure 6.4: Location of profiles acquired in the Kiel Bay, Aug. 2000 experiment

The OBS-stations have been deployed with a line and surface buoy, which is one possible cause for low frequency noise of high amplitudes but irregular occurrence in the seismic gathers evident in the data example presented in section 6.3 and fig. 6.13. It is less prominent at site A than at the other sites, which can be explained by the fact that the sea state was calm during the measurements at this site, while winds up to force $6 \mathrm{bft}$. caused more swell during the operating days at site $\mathrm{B}$ and $\mathrm{C}$. The removal of the arbritary distributed noise was not possible, since the noise is present at the frequency range of interest. It is therefore 
concluded, that either surface buoys should not be used at all, or need to be separated from the receiver system.

\subsubsection{Time synchronization of the source and receiver systems}

The synchronization between the source and receiver systems was one of the main issues in the practical pursue of the experiment. The autonomous OBS and OBH stations are continuously recording data during the experiment and have their internal quartz for time keeping which is synchronized with an GPS clock prior to the deployment and after retrieval of the system. The skew (the drift of internal clock in relation to GPS time) is measured and accounted for in the preparation of the data. Here, a linear clock drift is assumed, and for each shot the seismic trace is cut from the continuous recording, starting at the skew-corrected time of the shot. Since the clock drift is depending on temperature, this assumption will not be correct while the deployed system climatizes to the ocean bottom temperatures. Since the temperatures in the shallow water are not extreme, this fact is neglected for the OBH-system. However, presumably this was a mayor issue for the GeoPro-OBS-system, due to the fact, that the system had to be synchronized in the laboratory in Hamburg, hence synchronization could only be performed on the first and the fifth day of the experiment.

Another possible reason is the fact that several different GPS systems were used for the purpose of synchronization due to compatibility reasons. Apparently, the GPS time signal for the three different GPS systems used was not synchronous at all times. Possibly, the systems did not receive and use the signals from the same GPS-satellites, which would explain slight variations in position and time.

The synchronization of the OBC-recording was achieved differently, since the recording unit requires a trigger signal for each shot. The trigger signal was generated on FK Litorina and radioed to the recording vessel FK Polarfuchs just prior to the beginning of each profile. Here, the autonomous trigger unit on the recording system was synchronized, in order to maintain the constant shot rate at times without radio contact between the vessels. The skew of the radioed source trigger related to the autonomous recording trigger was measured prior to each synchronization. In laboratory conditions, it was measured that the radio transmittance of the source trigger pulse causes a constant delay in the order of $40 \mathrm{~ms}$ for the system used. Alternative methods to synchronize the two systems on the DCF77 radio signal transmitted from the atomic clock in Braunschweig were discarded due to the fact that here the DCF-receivers available, had a variable signal delay between 20 and $80 \mathrm{~ms}$ for two receivers of the same series. Hence, the radio trigger was superior due to the fact that the delay was more constant.

For future experiments with autonomous receiver stations it is suggested to abandon the requirement of the absolute time synchronization by performing a combined positioning and time synchronization measurement with a high frequency acoustic system mounted to the 
receiver system. Since we require an accurate absolute position to obtain correct sourcereceiver distances for further processing, a high frequency measurement with several known shot positions to determine the receiver position by travel time inversion is suitable. It is just a numerical enhancement of the inversion process to include the absolute time as an unknown parameter into the inversion process. Such an experiment could be combined with high resolution seismic measurements to obtain the detailed stratigraphy and additional constraints to the layer thicknesses.

\subsubsection{Determining and achieving the required shot interval}

The choice of the shot interval has to be adapted to the requirements for the wave field transform as discussed in section 4.3, but practical limitations also constrain this acquisition parameter. The throughput of compressed air by the airgun source is determined by the shot interval, the chamber volume and the air pressure in the chamber. The amount of compressed air used by the airgun has to be produced by the compressor system available on the vessel. By adapting the pressure reduction unit of the airgun system to be fitted to a conventional electric compressor available on FK Litorina, we could produce the required amount of air without the need of a large hydraulic compressor, generally used in airgun seismic experiments. This made the operation on a small and maneuverable research vessel of less than 30 $\mathrm{m}$ length possible. However, we therefore have a maximum average throughput limited to $\approx 1500 \mathrm{l} / \mathrm{min}$. With a buffer in form of pressure tanks ( 2 x 501 was used), we can use more compressed air during the profile, which is refilled during the turns between profiles.

The air pressure in the airgun chamber as well as their volume determines the amount of seismic energy discharged into the water. Tests with airguns of $0.31,0.61$ and 1.21 with pressures of 60 to 120 bars revealed that the amplitudes of the Scholte wave signal increased for larger chamber volume, but so does the required amount of compressed air. The energy release of the 0.61 airgun at 70-80 bar was sufficient for the analysis of the dispersive Scholte waves, presumably due to the shallow water conditions.

With this configuration we could shoot a 0.61 airgun every 10 seconds for nearly two hours on one profile. This is long enough to cover a profile length of 1 nauticle mile (nm). Hence, we have up to $0.5 \mathrm{~nm}(\approx 900 \mathrm{~m})$ to either side of the deployed station or nearly $2 \mathrm{~km}$ if a continuous profile is started or ended at the deployed station.

\subsection{Data examples, acquisition and preprocessing}

A representative common-receiver-gather (CRG) from the OBS station at site $\mathrm{A}$ is shown in fig. 6.5a with offset dependent scaling in order to compensate geometrical spreading. The highest amplitudes are observed in the reflection events and the first arrivals, which are 
thought to be an interference of the direct wave and the sea floor reflection/refraction due to the shallow water geometry.

The Scholte interface wave is clearly visible once the high amplitude reflection events have separated at later arrival times exceeding offsets of $50 \mathrm{~m}$. It is easily identified due to its low velocity of propagation and distinctively lower frequencies compared to the direct and reflected arrivals in the seismic wave field. This enables to enhance the Scholte wave amplitudes in the CRG by simple low pass frequency filtering. To account for variations in shot strength, each seismogram is normalized to its maximum amplitude within each seismogram section, prior to the dispersion analysis or wave field transforms to be applied. In some cases $\mathrm{f}-\mathrm{k}$-domain filtering may enhance the signal of the Scholte wave energy compared to reflected or ambient noise energy in the gather, but generally no other preprocessing has been applied.

\subsubsection{Comparison of different receiver systems}

In order to compare the potential of recording Scholte wave energy with either of the three receiver systems available, low-pass filtered seismic CRGs from the three sensor systems with equivalent offset ranges are shown in fig. 6.7. The locations are not identical, but within a range of $400 \mathrm{~m}$ apart, hence the sub bottom properties at each location may vary slightly.

The $\mathrm{OBH}$ registration suffers from strong low-frequency reverberations dominantly in the near offset range, which do not occur on the other two sensors. Here, the construction of this particular $\mathrm{OBH}$ might be the cause for these reverberations, since the hydrophone is mounted on the stand in between the buoyancy body and the sea bed, approximately half to three quarters of a meter above the sea bed. The characteristic of the reverberation is most dominant in the the wavelet of the first arrival of the OBH. These wavelets of each of the three receiver systems and the corresponding frequency spectra are shown in fig. 6.6. Other possibilities for such reverberation have been discussed by (Osler \& Chapman, 1998, e.g.).

Also remarkable is the variation of the amplitude and phase of the direct wave of the OBC registration. Here, the section presented in fig. $6.7 \mathrm{c}$ is a concatenation of three commonshot-gathers with varying shot distance in order to cover the same offset range as shown in the OBS and OBH sections. The concatenation is at $115 \mathrm{~m}$ and $230 \mathrm{~m}$ offset of the section, where some traces of the different shots overlap in the offset range. We observe a variation in the phase of the filtered first arrival near offset $220 \mathrm{~m}$, which corresponds to identical receiver locations. The variation of the receiver coupling along the receiver spread could cause such an amplitude variation.

Since we require repeatability of the influence of source and receiver on the recorded seismograms, such variations in the receiver coupling complicates the analysis of the data. It was therefore concluded, that the ambiguity between source-receiver related and sub-surface inferred variations of the seismic wave field is reduced by using just one single sensor for each interpretation and inversion of the dispersive wave field. However, if the variation in 


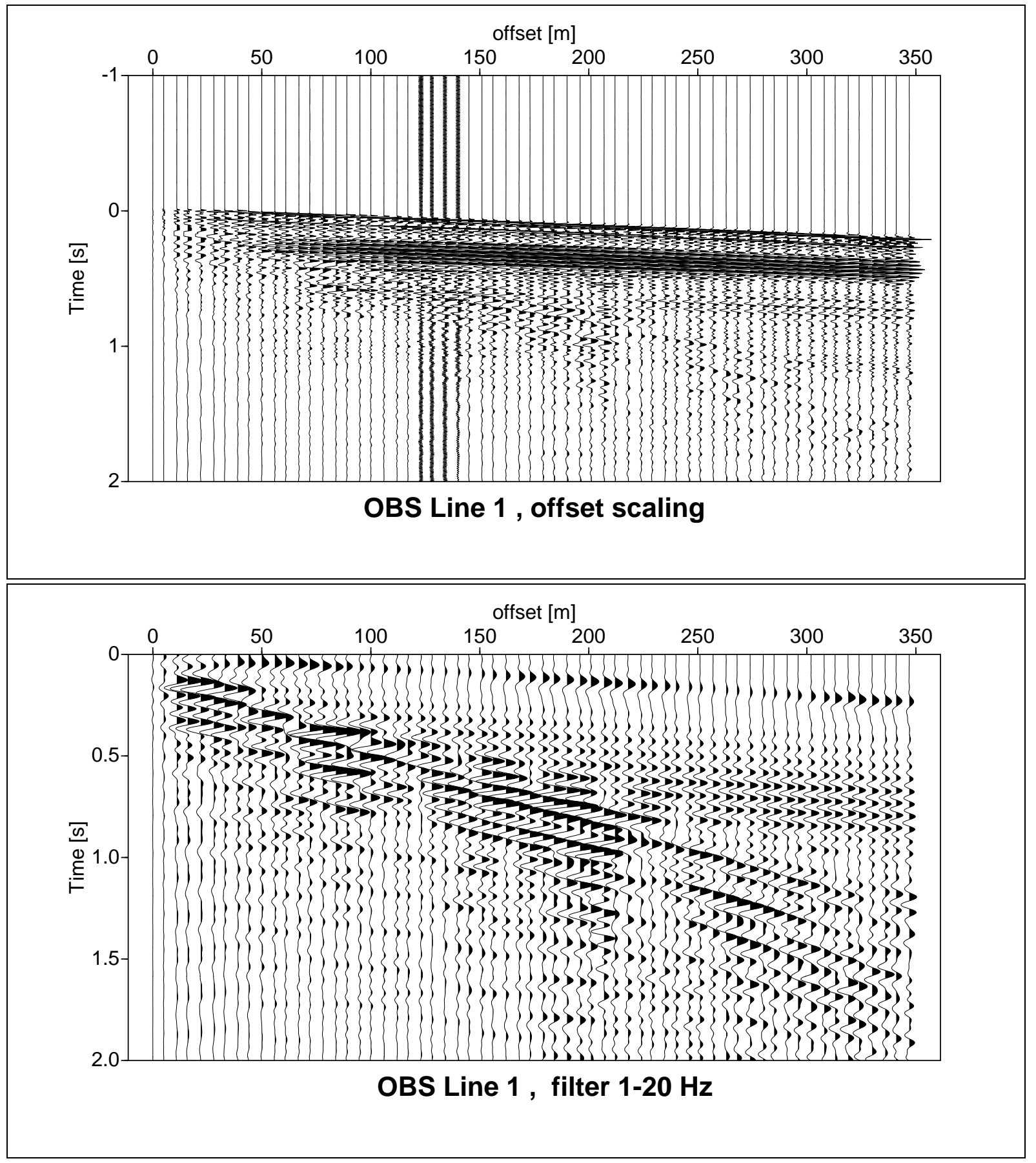

Figure 6.5: Common-receiver-gather of the OBS prior and after the application of the lowpass filter at $20 \mathrm{~Hz}$. 

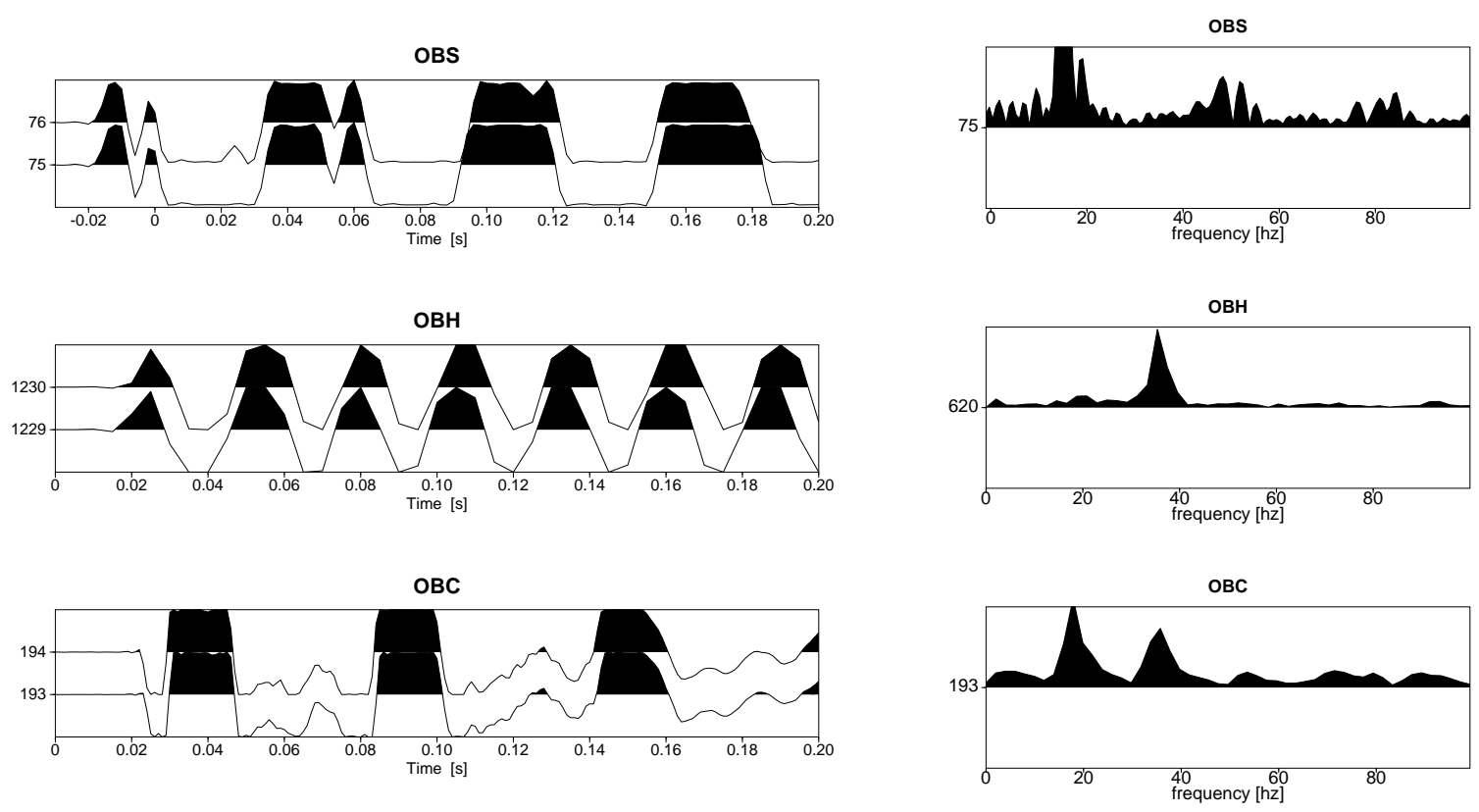

Figure 6.6: Comparison of the wavelets (left) and the corresponding frequency spectra (right) from the OBS, OBH and OBC. 


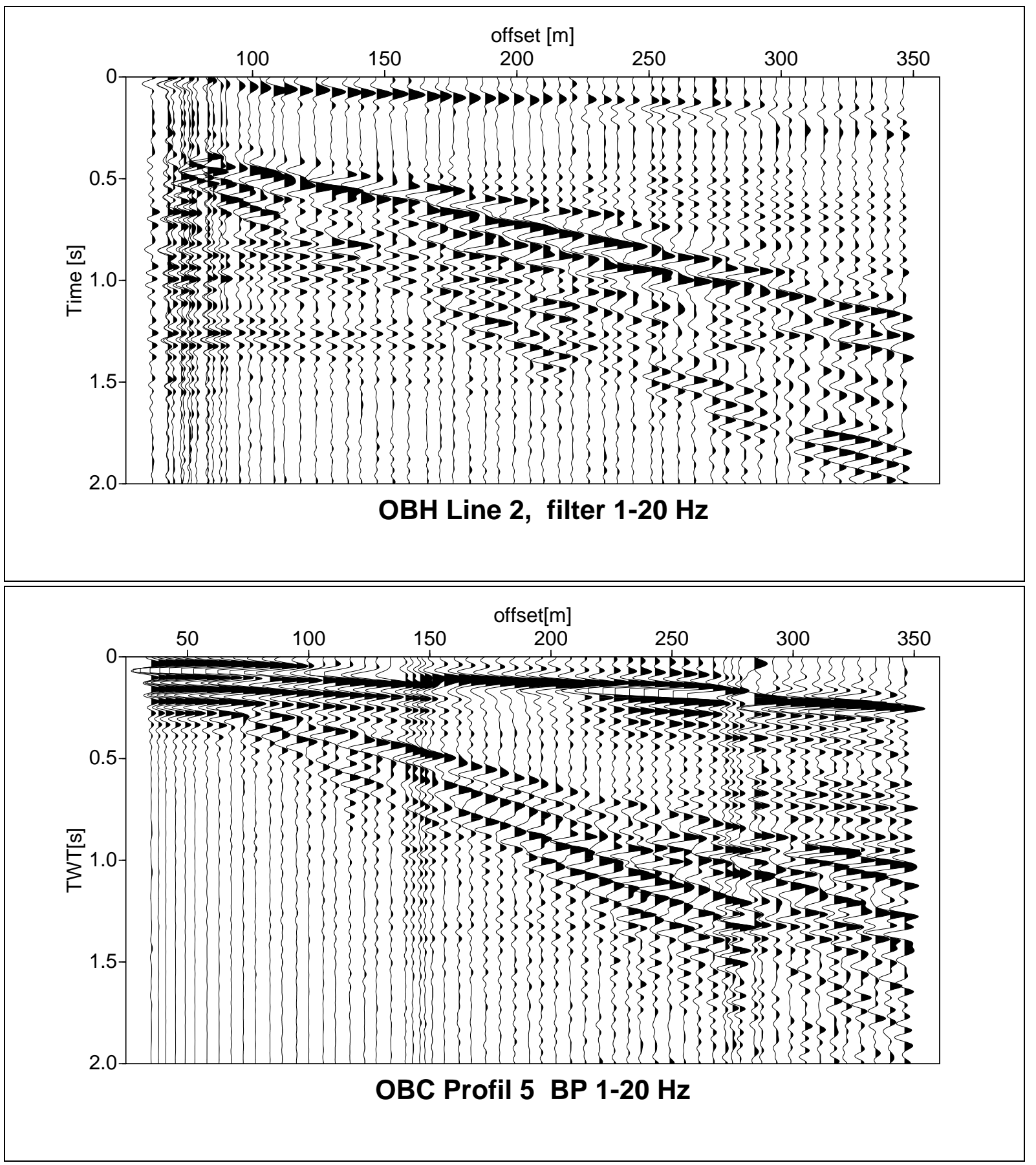

Figure 6.7: Comparison of seismic shot gathers of various ocean bottom sensors 
receiver coupling could be quantified by an independent measurement, the use of a receiver spread could overcome the limitations due to the stationary-receiver geometry.
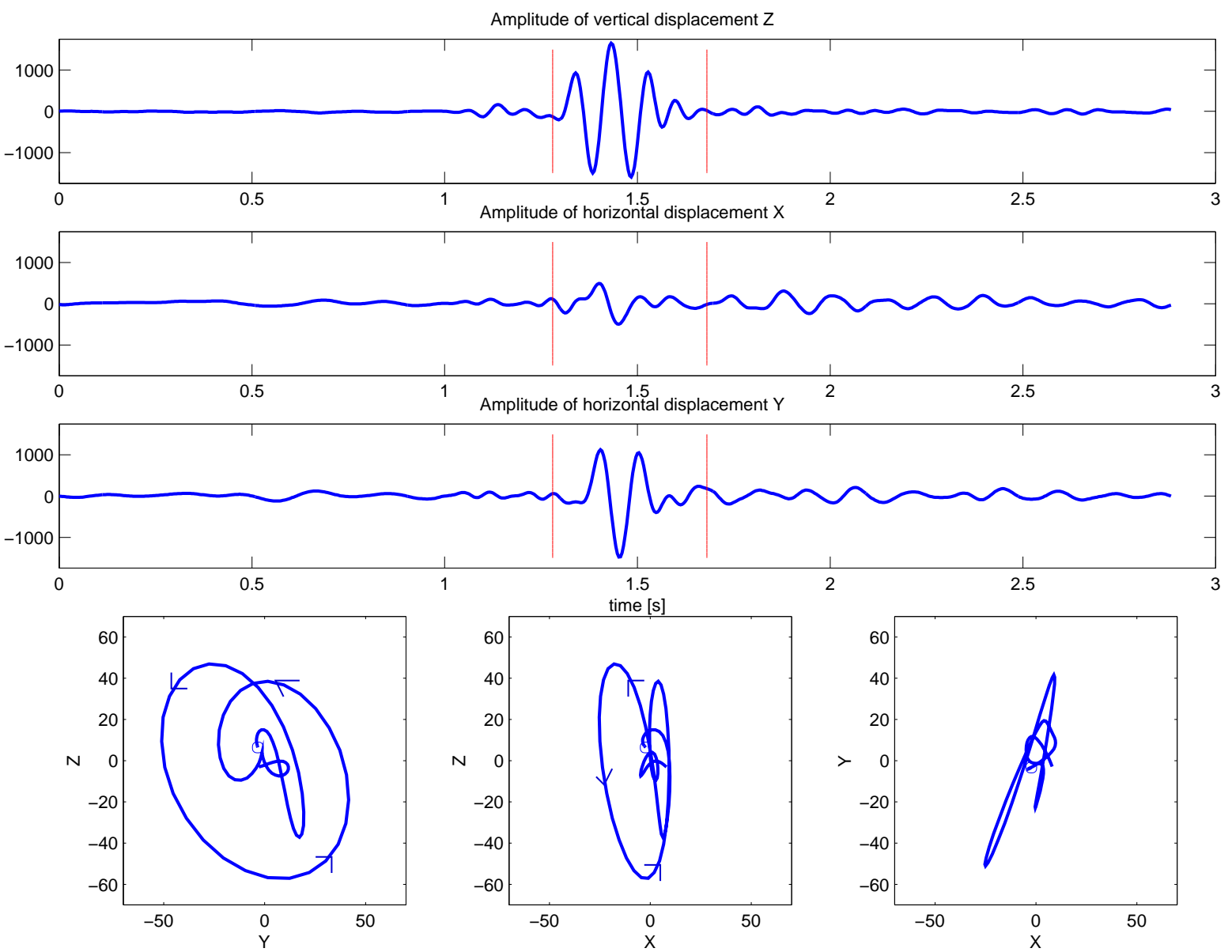

Figure 6.8: The particle displacement for the three components ( $\mathrm{z}=$ vertical and $\mathrm{x}, \mathrm{y}=$ horizontal) of the seismometer recording (OBS) and the hodograms of the particle motion projected in the three planes (from Kugler, 2001)

The three geophone components of the OBS recording enable to reconstruct the particle motion at the sea floor. This is a valuable advantage against the two other recording systems used. Hodograms of the particle trajectory can be calculated from the particle velocity measured in the geophones. This is achieved by integration in the Fourier-domain to obtain the particle displacements of a signal corresponding to the Scholte wave energy (Fig. 6.8). The elliptically polarized motion of the particles is clearly visible in the lower three images of the projections of the 3-D particle motion into the three coordinate planes. 

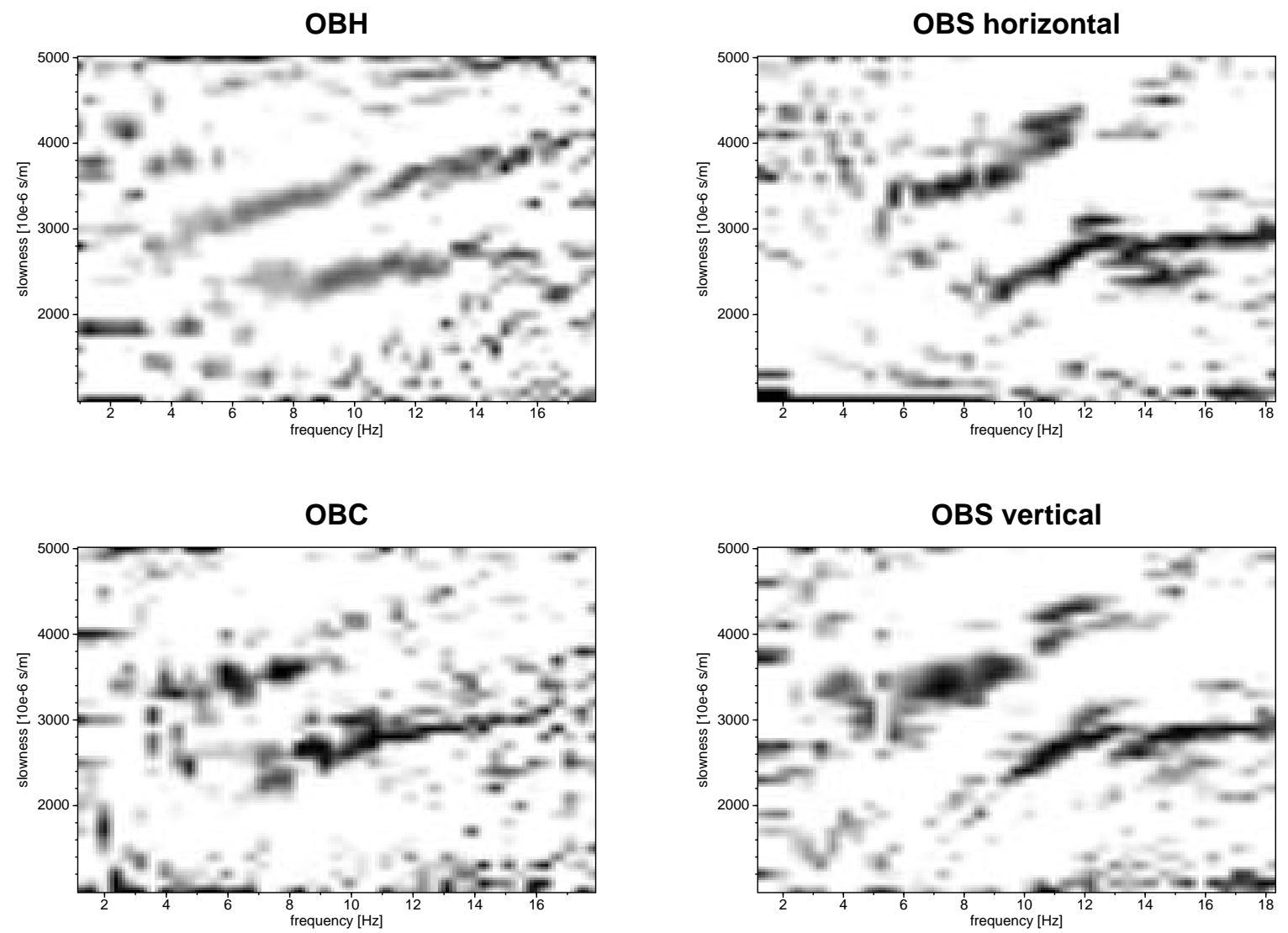

Figure 6.9: The results from the wavefield transform into the frequency-slowness domain of the shot gathers shown in fig. 6.7 . 
In the wave field transformed spectra of the CRGs using the time-domain slant-stack algorithm, we observe correlated energy presumably related to the fundamental and first higher modes of the Scholte wave (Fig. 6.9). The geophone recordings of the OBS reveal a slightly superior image of the modes. Noise problems encountered at site B during the second experiment have been more severe on the geophone components. The amplitude level of Scholte wave energy in the hydrophone recordings is about $20 \%$ lower compared to the vertical component of the geophone. This amplitude ratio of 0.8 for the Scholte wave energy at low frequencies is large compared to the amplitude ratio of 0.1 for the wave energy at higher frequencies (reflected, refracted and converted waves). Therefore, we conclude that the low frequency interface waves are well observable on the hydrophone component.

\begin{tabular}{|c|c|}
\hline OBS & $\begin{array}{l}\text { - the particle motion can be resolved from the geophone compo- } \\
\text { nents } \\
\text { - high variability in receiver coupling } \\
\text { - more liable for noise and resonances of the recording system }\end{array}$ \\
\hline$\overline{\mathrm{OBH}}$ & $\begin{array}{l}\text { - receiver signal distortion is less dominant and more stable } \\
\text { - Scholte wave energy is only slightly reduced compared to vertical } \\
\text { component recordings. }\end{array}$ \\
\hline OBC & $\begin{array}{l}\text { - multi channel recording enables to check source and receiver re- } \\
\text { peatability and coupling } \\
\text { - variations in receiver coupling must be accounted for } \\
\text { - combination of common-shot and common-receiver recordings } \\
\text { can supplement to facilitate the acquisition with adequate spacial } \\
\text { sampling. }\end{array}$ \\
\hline
\end{tabular}

Table 6.1: Comparison of the sensor systems

The following advantages and disadvantages of different ocean bottom sensor systems are summarized in table 6.1. 
In order to determine, whether variations of the dispersion due to changes in the sub bottom sediment properties can be revealed by the p-f-spectra, the measurements at the different geological sites are analyzed.

\subsubsection{Scholte wave dispersion at different geological sites}

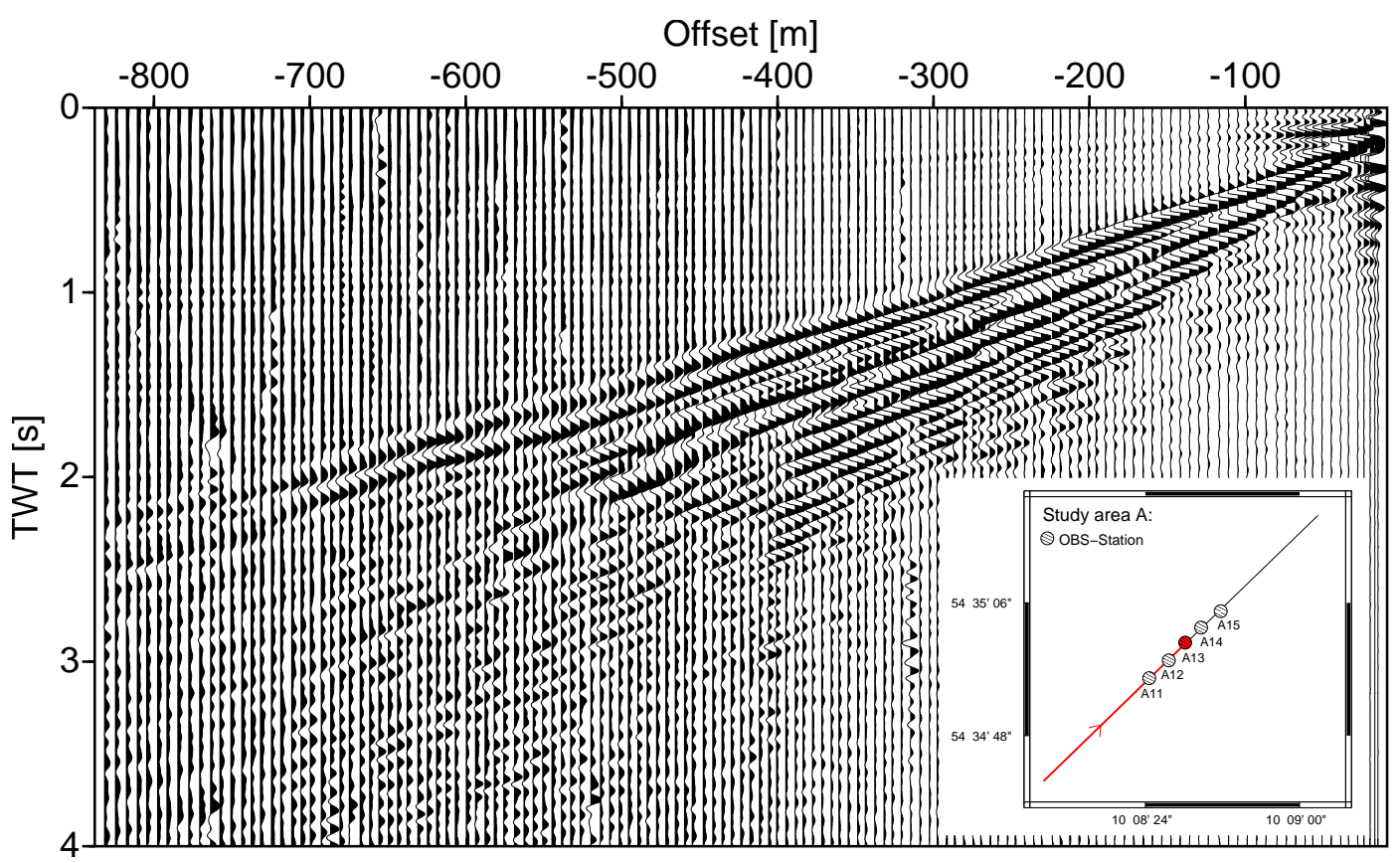

Figure 6.10: Common-receiver-gathers recorded at site A. The body wave arrivals have been suppressed by low-pass filtering, while the two modes of the Scholte wave energy propagating at low group velocity are very clear (from Kugler, 2001).

Examples of the common-receiver-sections acquired during the August 2000 seismic experiment in the Kiel Bay are described in the following sections. The data sets are classified according to the geological settting at the site:

site A - Glacial till (OBS-A13, Fig. 6.10),

site B - Holocene mud (OBS-B31, 6.13, top) and

site C - Sandy-slit sea bed (OBS-C14, 6.13, bottom).

The analysis and inversion of the seismic data from these sites was carried out in the scope of the master project of Simone Kugler, embedded in the frame of the Scherseis project (Kugler, 2001). 


\section{Site A - Glacial till}
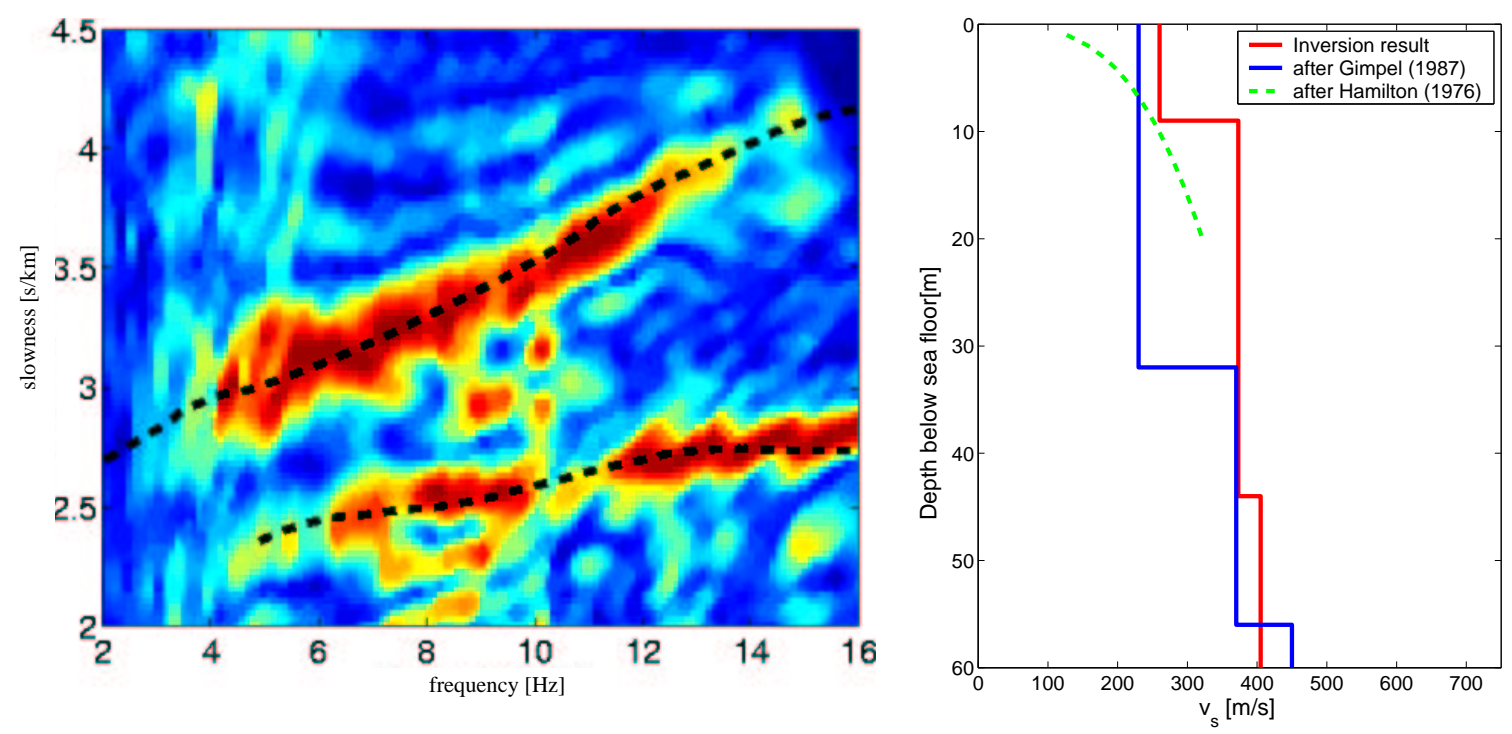

Figure 6.11: Inversion results from site A compared with data from previous investigations (from Kugler, 2001)

In the seismic common-receiver-section from site A, two dispersive modes can be identified in the slowness-frequency domain in figure 6.11a as well as in the time domain. At this site, the p-f spectra differ significantly for the individual OBS locations. Variations are also significant for the two sub-profiles at either side of each of the OBS stations. This lateral variation in the seismic spectra is caused by lateral variations of the subbottom properties. The inversion procedures introduced in section 5.2 have been applied to derive the shear wave velocity profile at this site (Bohlen et al., 2003; Kugler, 2001). From the inversion of the fundamental and first higher modes of the seismic wave field, we obtain a two-layer model with shear wave velocities of 260 and $380 \mathrm{~m} / \mathrm{s}$ respectively (Fig. 6.11b). The resolution analysis showed that the velocity of the top layer is given to less than $10 \%$ error. The error increases with depth, but remains less than $25 \%$ up to $35 \mathrm{~m}$ depth below sea bed (Fig. 6.12). The inversion result for the layer thickness of the top layer is given to $9 \mathrm{~m}$ with $25 \%$ error. It should be noted, that a gradient of increasing shear wave velocity with depth at a rate of $15 \mathrm{~m} / \mathrm{s}$ per one meter depth would probably fit the data just as well. The inversion result is shown in fig. 6.11 together with results from the measurements and analysis of PS-converted waves by Gimpel (1987) from the nearby Stollergrund study area (Gimpel, 1987). In comparison, both results are in good agreement. The velocities are similar and the thickness of the layers is likely to differ between the two sites by the range observed in the data. Also indicated is the empirical relation obtained from laboratory measurements by Hamilton (1976) for coarse sands. 


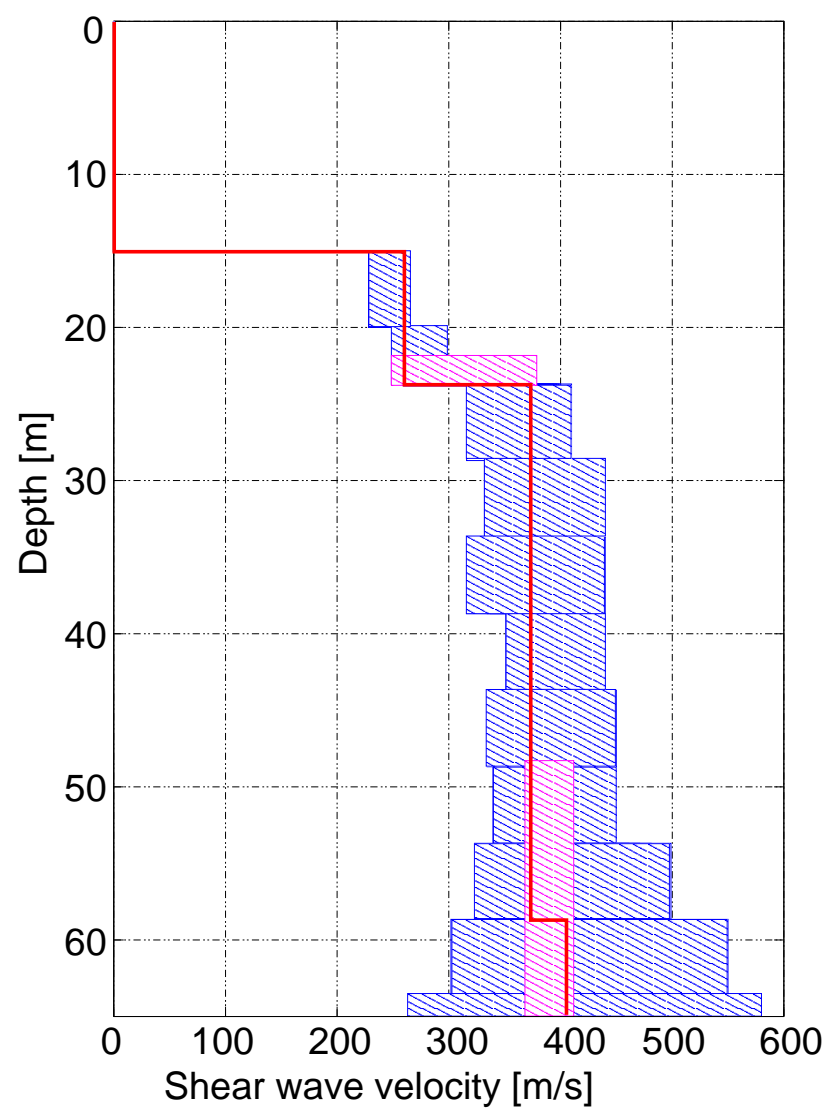

Figure 6.12: Resolution analysis for the inversion result for Site A was performed by the "rubber band" test, i.e. by successive variation of the shear wave velocity value in each of the $5 \mathrm{~m}$ thick layers producing an increase in misfit of $10 \%$. 


\section{Site B - Holocene mud}

The seismic data at site B was of poor quality with respect to very high amplitude noise in the frequency range of concern. The noise is arbitrarily distributed along the continuous record of the OBS stations, regardless of the timing of the seismic source signal. At this site a higher sea state and windy weather conditions were encountered compared to the measurements at the other two sites. Therefore the noise is believed to relate to the surface buoy attached to the OBS system, which could produce significantly more noise signals at these higher sea states.

Note, however, the coherent low frequency signal observed in the very first three seismograms of the common-receiver gather in fig. 6.13b. This could indicate the presence of a Scholte wave of very low velocity of propagation but high attenuation as expected in very soft muds, known to be present at this site. No dispersive modes could be detected in the wave filed spectrum of this site. Therefore the inversion could not be performed. However, similar seismic wave forms could be produced in the synthetic seismograms in figure 6.14. The seismogram section was computed for a model representing a soft surfical layer of mud overlaying harder sediments (e.g. the glacial till observed at site A). The frequency of the dominant dispersive signal related to the Scholte wave is below $4 \mathrm{~Hz}$, hence below the eigenfrequency of the seismic sensors used in the experiment.

\section{Site C - Sandy-silt sea bed}

At the third site the data was of better quality, but of very complex nature with respect to the dispersion observed in the seismic wave filed. The $p$-f spectrum corresponding to the seismic section of this site is shown to the left of fig. 6.15. Here, the spectrum could not be analyzed with the inversion algorithm due to the complex nature of the spectrum. A synthetic spectrum generated for a model including a low velocity zone is shown in fig. 6.15a (middle) with the corresponding model in $b$ (right). Hence, a low velocity zone could be a possible cause for the complex image in the $\mathrm{p}-\mathrm{f}$ spectrum.

\subsection{Conclusions}

In shallow water conditions a small surface towed airgun excites Scholte waves with sufficient energy to be recorded with ocean bottom sensors, hydrophones as well as geophone components. Using the 0.61 airgun, the Scholte wave field can be separated by simple low-pass frequency filtering below $20 \mathrm{~Hz}$.

The use of seismometer stations is advantageous, since the particle motion diagrams may help to identify the presence of elliptically polarized Scholte waves. Yet, it is also shown, 

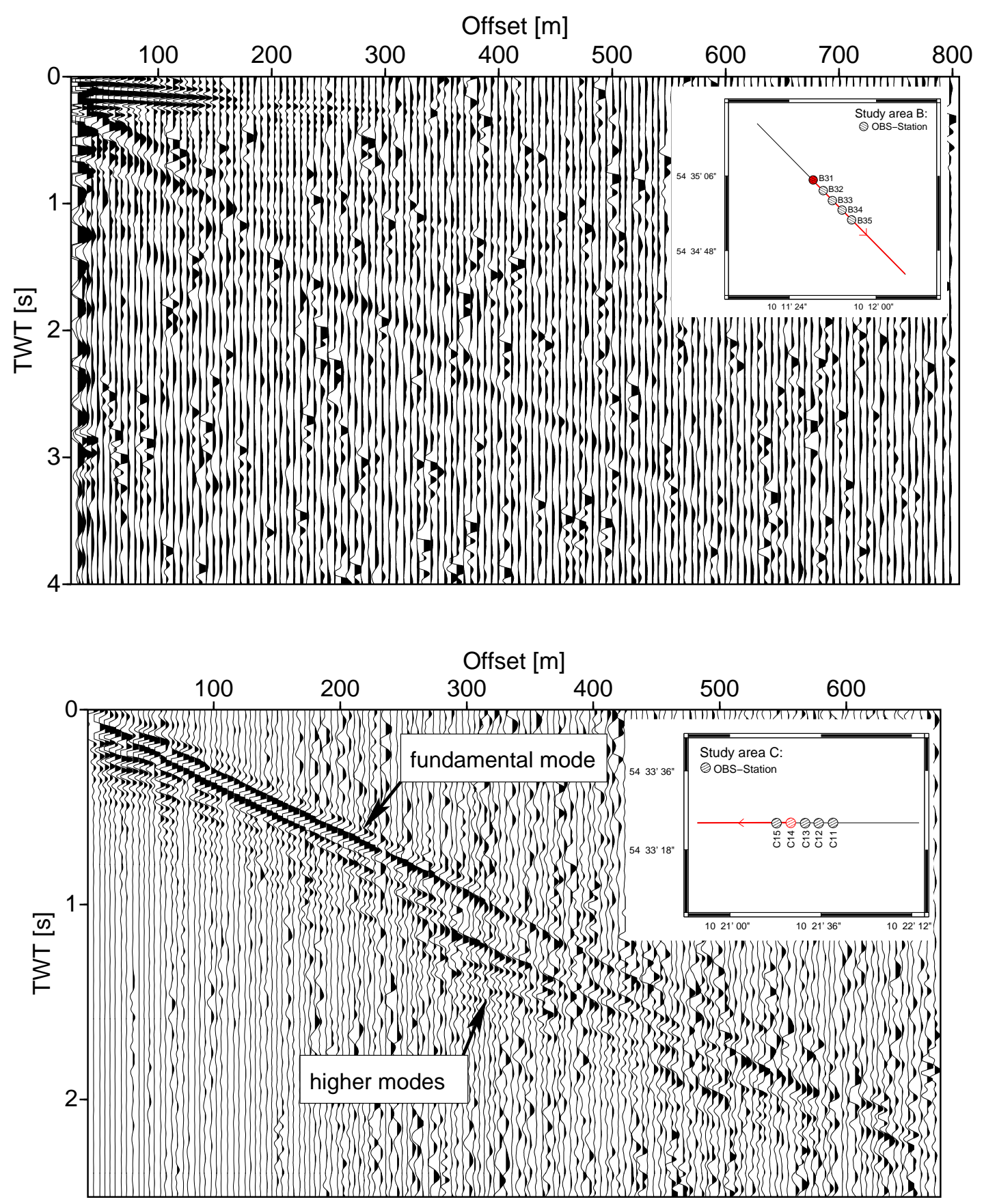

Figure 6.13: The common-receiver-gathers from sites B (top) and C (bottom) are not as clear as the corresponding seismic section of site A (from Kugler, 2001). 

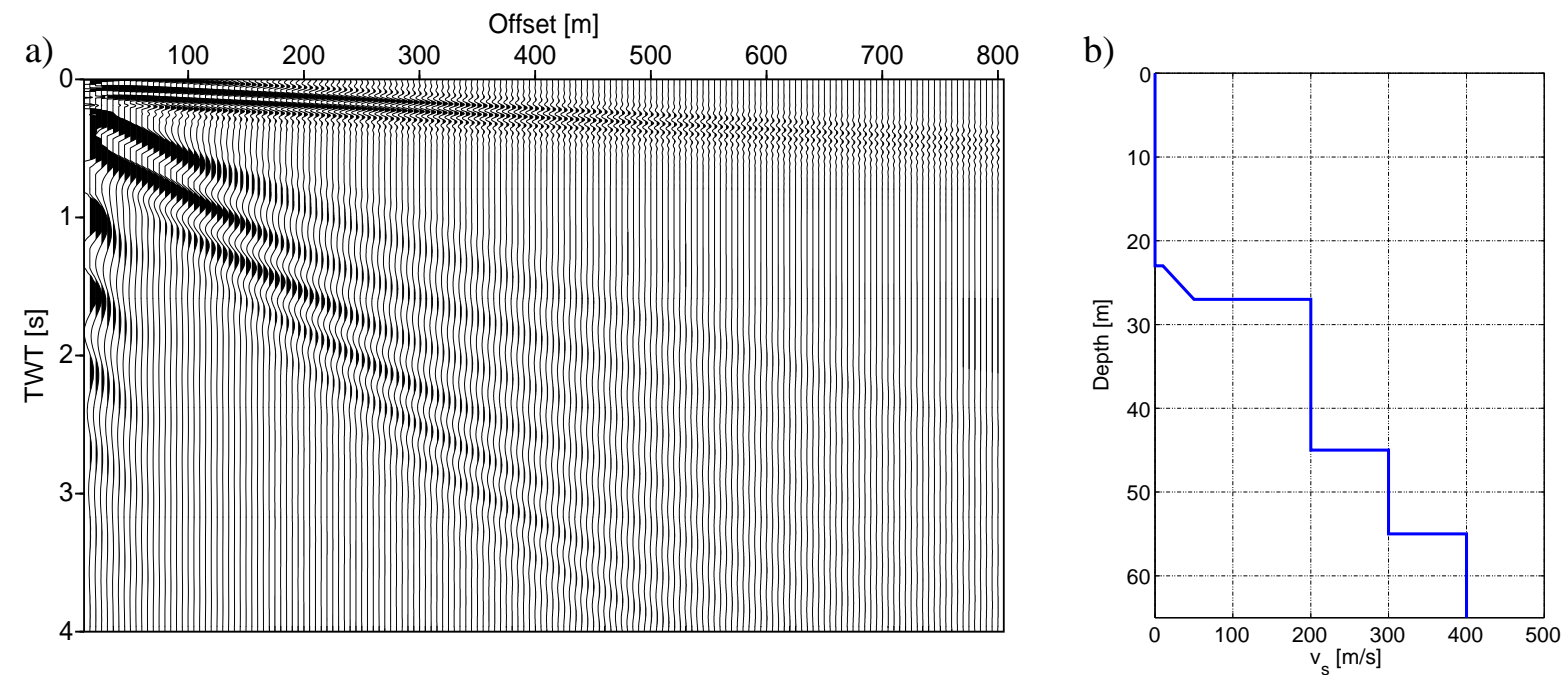

Figure 6.14: Synthetic seismogram and the corresponding model possibly resembling the geological situation at site B (from Kugler, 2001).

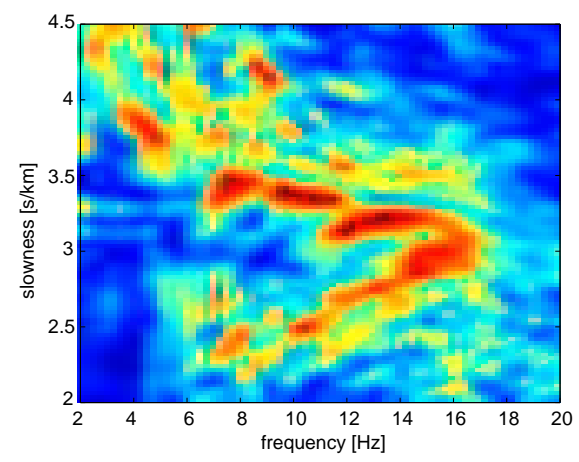

a)

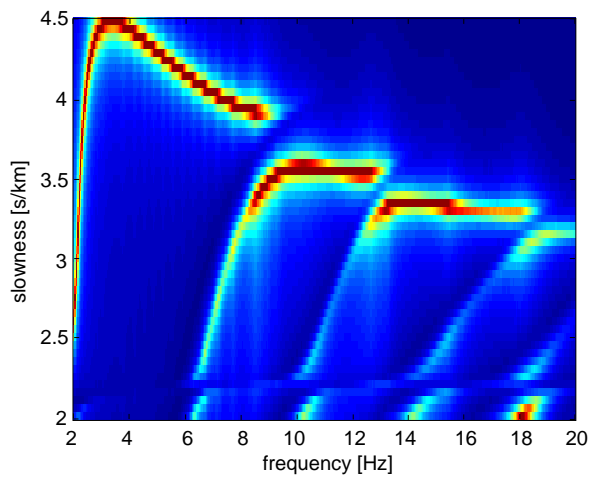

b)

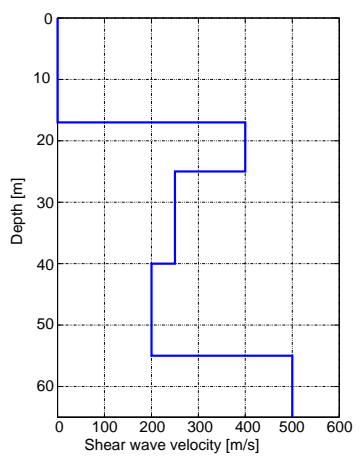

Figure 6.15: The measured p-f-spectrum from site $\mathrm{C}$ (right) compared with a synthetic spectrum (center) corresponding to the shear wave velocity model on the right (from Kugler, 2001). 
that the hydrophone component data had sufficient Scholte wave energy and is generally less liable to noise and shot-to-shot variability due to receiver coupling or device-induced noise.

For future experiments I propose additional high frequency measurements in order to facilitate the data analysis procedures. High frequency records could be used to simultaneously determine the receiver position as well as the absolute time delay of the recording units. These measurements could be performed in conjunction with high resolution seismic to determine the stratigraphic sequences. The recording units of the ocean bottom stations would need to record at significantly higher sample intervals for these measurements.

At one of three sites the shear wave velocity structure of shallow marine sediments could be inferred from the dispersive seismic wave field of Scholte waves, by using an inversion procedure newly developed within the scope of the Scherseis project and the master thesis of Simone Kugler. The limitations of the method have been encountered at the two other sites. Firstly, due to the very low velocity structure at site B, the seismic wave field was not sampled adequately. Furthermore is the frequency range of interest at this site below the receiver specific lower limit. Secondly, the complexity of the subsurface structure can limit the applicability of the inversion method. Therefore the development of more sophisticated inversion procedures for these complex structures are continuously investigated. 


\section{Chapter 7}

\section{Acoustic Guided Waves - Marine Permafrost in the Laptev Sea, Siberia}

\section{Contents}

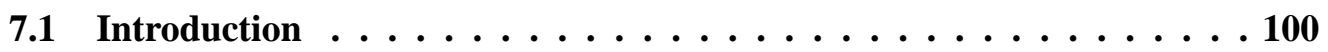

7.2 Multichannel Seismic Data $\ldots \ldots \ldots 10 \ldots \ldots$

7.2 .1 Study Area . . . . . . . . . . . . . . . . . . . 101

7.2.2 Seismic Lines and Acquisition Parameters . . . . . . . . . . 103

7.2.3 Data Example . . . . . . . . . . . . . . . . . . 103

7.2.4 Regional Variation of Dispersion Curves . . . . . . . . . . . . . . . 105

7.3 Sensitivity of Seismic Parameters of Dispersive Guided Waves . . . . 110

7.3.1 Reference Model . . . . . . . . . . . . . . . . . . 111

7.3.2 Sensitivity of the Acoustic Guided Modes to Variations of Sediment Properties . . . . . . . . . . . . . . . . 111

7.4 Inversion . . . . . . . . . . . . . . . . . . 118

7.4.1 Inferring Seismic Parameters from Shot Gathers in two Stages . . . 118

7.4 .2 Inversion Results . . . . . . . . . . . . . . . . . . . 121

7.4 .3 Resolution Analysis . . . . . . . . . . . . . . . . . . . . 122

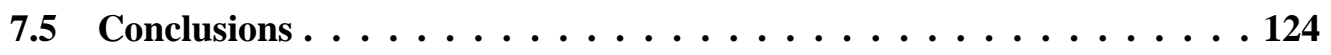




\subsection{Introduction}

\section{Characteristics of submarine permafrost in the Laptev Sea}

The Arctic shelves which have seen transitions from sub-aerial to marine conditions during the glacial and interglacial stages are the only places where submarine permafrost conditions are known. Permafrost resides wherever the mean annual temperature of material (e.g. soil, rocks, sediments) is below $0^{\circ}$ centigrade for several years. There is evidence from seismic data as well as from cores from the Laptev Sea, that permafrost occurs widespread on the broad and shallow continental shelf of the Laptev and East Siberian Seas. It is believed, that the permafrost formed in sub aerial conditions in the glacial stages, when most of the continental shelf emerged due to the lower sea levels. The extend of submarine permafrost is therefore believed to be induced from the last sea level low stands and depending on the net thermal heat flux in the area. Mapping the extend of the permafrost today provides therefore important input parameters for the estimation of thermal heat flow and conductivity parameters in the area and therefore enables to investigate climate impact on gas hydrate stability zones (Deslile, 1998).

\section{Can seismic data provide additional (complementary) information on the sediment structure?}

Large scale seismic surveys to investigate the tectonic setting and the extend of the Laptev Sea Rift in relation to the mid ocean ridge (Gakkel Ridge) have been performed by the BGR in 93, 94 and 97 (Franke et al., 2001). The presence of large amplitude dispersive wave trains in the seismic shot gathers made the conventional reflection seismic processing more complex than expected. Moreover the question of the cause and the usefulness of these wave phenomena in the seismic data arises. The dispersive waves have been interpreted to represent acoustically guided waves due to the presence of a hard sea bottom at shallow water depth. The water column presents the wave guide and I discuss how additional parameters of the sub bottom sediment structure can be determined from the dispersive waves. Moreover it is possible to use conventional reflection seismic data to estimate the depth variation of the shear wave velocity of the upper few tens of meters in the sediment, a seismic parameter which is not easily determined otherwise.

\section{Potential of dispersive guided waves to derive Vs depth profile}

The analysis of dispersive seismic waves to derive the variation of shear wave velocity with depth has been applied to surface wave data in many occasions. It is common practice in 
seismology to invert the Rayleigh wave dispersion for sub surface seismic parameters including shear wave velocity and attenuation coefficients. Also in small scale geotechnical problems or in non-destructive material testing the dispersion analysis of interface waves (SASW-Method) has become very popular in the last few decades.

The investigation of guided waves, which exhibit multi-modal dispersion relating to the thickness of the wave guide and the wavelength excited, has not been done to a great extend. Here I present a case study, where the analysis of dispersive guided waves can add complementary information to the normal reflection seismic data analysis.

\section{Case Study Outline}

To begin a data example of the reflection seismic survey of the BGR in 1997 is presented as well as the application of a full wavefield transform to reveal the dispersive characteristic of the guided waves of the seismic common shot gathers (Sec. 2). Then synthetic seismic wavefield computations of a layered (1-D) reference model to show the characteristics of the guided wavefield and the sensitivity to the seismic parameters of interest (Sec 3). Finally an inversion method to infer the variation of sub-bottom seismic parameters with depth from the recorded dispersive seismic data by full wavefield inversion in the slowness-frequency domain (Sec 4) is presented and the inversion results are compared with independent information from other publications (Sec 5).

\subsection{Multichannel Seismic Data}

For investigations towards the understanding of the tectonic setting of the Laptev Sea study area, more than $4000 \mathrm{~km}$ Multi-Channel-Reflection-Seismic (MCS) lines along with several wide-angle reflection seismic lines with Ocean-Bottom-Hydrophone (OBH) stations have been acquired in 1997 (Hinz et al., 1997). Near surface reflections and high velocity refraction arrivals indicated the presence of a high-velocity layer interpreted as submarine permafrost. The high velocities at near surface complicate the refraction analysis of the near surface and sub permafrost layers and we seek additional information to the depth profiles of the seismic velocities.

\subsubsection{Study Area}

The Laptev Sea is a shallow Randmeer of the Arctic Ocean, surrounded by the New Siberian Islands and the East Siberian Sea to the east and the Taimyr Peninsula to the west. The Laptev Sea is covering a broad continental shelf with water depth from only $10-100 \mathrm{~m}$ for $\approx 300 \mathrm{~km}$ from the Lena River delta in the south to the drop into the Eurasian Basin of the Arctic Ocean 


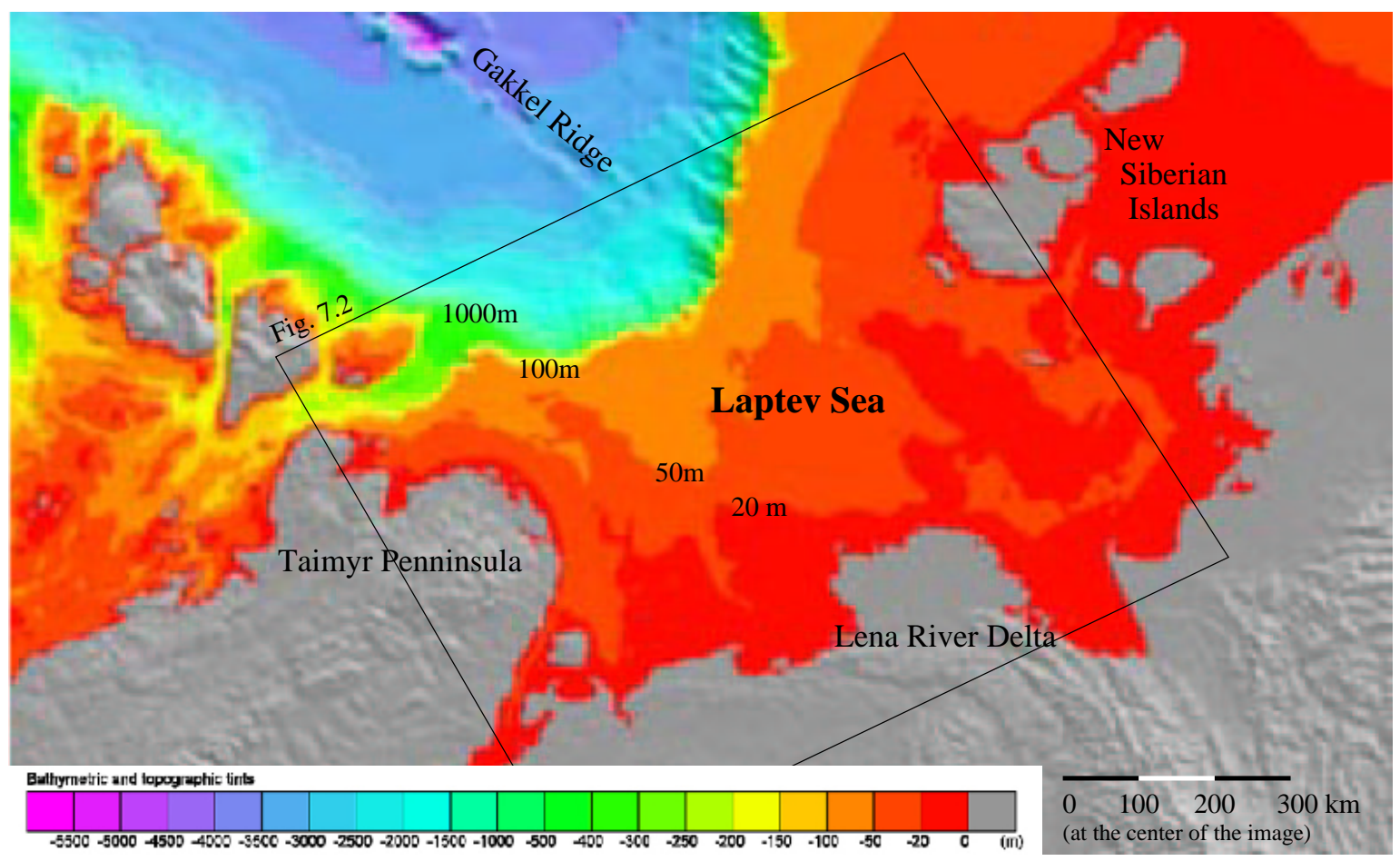

Figure 7.1: The bathymetry map of the Laptev Sea shows the large plateau area of the shelf with water depth below $100 \mathrm{~m}$ up to $300 \mathrm{~km}$ and a sudden drop into deep waters. The tectonic setting is indicated by the Gakkel Ridge, i.e. the mid-ocean ridge and boundary between the North American and Eurasien plates, and the mountain ranges on shore. 
in the north. Due to the shallow depth of less than $100 \mathrm{~m}$ the continental shelf was exposed in cold stages and phases of marine regression and continental permafrost developed. The influence of climate and heat flux changes have been investigated by Delisle et.al. in detail (Deslile, 1998).

The unique plate tectonic setting of the North American and Eurasian plates separated in the Eurasian Basin just north of the broad continental shelf of the Laptev Sea study area gave rise to detailed deep reflection seismic investigations. In 1997, the Federal Institute for Geosciences and Natural Resources (BGR, Hanover), in cooperation with Sevmorneftegeofizika (SMNG), Murmansk, acquired $4000 \mathrm{~km}$ of multi-channel reflection seismic (MCS) lines and several wide-angle reflection/refraction lines in the area to illuminate the tectonic setting. A small subset of this data was used for the investigation of further potential of information from the dispersion analysis presented in this work.

\subsubsection{Seismic Lines and Acquisition Parameters}

The acquisition parameters of the seismic data is summarized in fig. 7.3. The location of the subsets of the Lines 1, 5, 20 and 21, each located in the vicinity of Ocean Bottom Hydrophone $(\mathrm{OBH})$ stations of the wide-angle seismic data, are marked in fig. 7.2. The refraction analysis of the OBH-data performed by the BGR can provide additional information to verify/calibrate the results from the dispersion analysis.

The first seismic line starts in very shallow water and crosses the main discharge channels of the Lena river. The water depth in the location of the subset discussed averages to $20 \mathrm{~m}$. The average water depth of the other subsets of lines 5, 20 and 21 is in the range of $40 \mathrm{~m}$. Line 1 has the most dominant dispersion features with additional dispersive interface waves present in both, the MCS and OBH seismic data. Yet, I want to demonstrate the analysis method on the data example from line 21 on which the variation of the dispersion characteristic along the line is more pronounced.

\subsubsection{Data Example}

The shot gather presented in fig. 7.4 shows refracted arrivals with apparent velocities of 3.6 $\mathrm{km} / \mathrm{s}$ which are interpreted as the top of the permafrost zone. Dominant reflection arrivals can be identified at $2 \mathrm{~s}$ two-way-travel-time (twt) while a high-frequent "direct wave" with velocity of $1.4 \mathrm{~km} / \mathrm{s}$ may mask other reflection events with earlier arrival times. The time-reduced shot gather (a reduction velocity of $1.5 \mathrm{~km} / \mathrm{s}$ was applied) presented in fig. 7.5 reveals, that the direct wave is a superposition of dispersive modes. Clearly visible are the phases of the mode marked by the arrows correlating with higher velocity than the propagation velocity of the mode, hence a normal dispersive propagation pattern. The transformation of the wavefield into the slowness-frequency domain, using a frequency domain slant-stacking algorithm as 


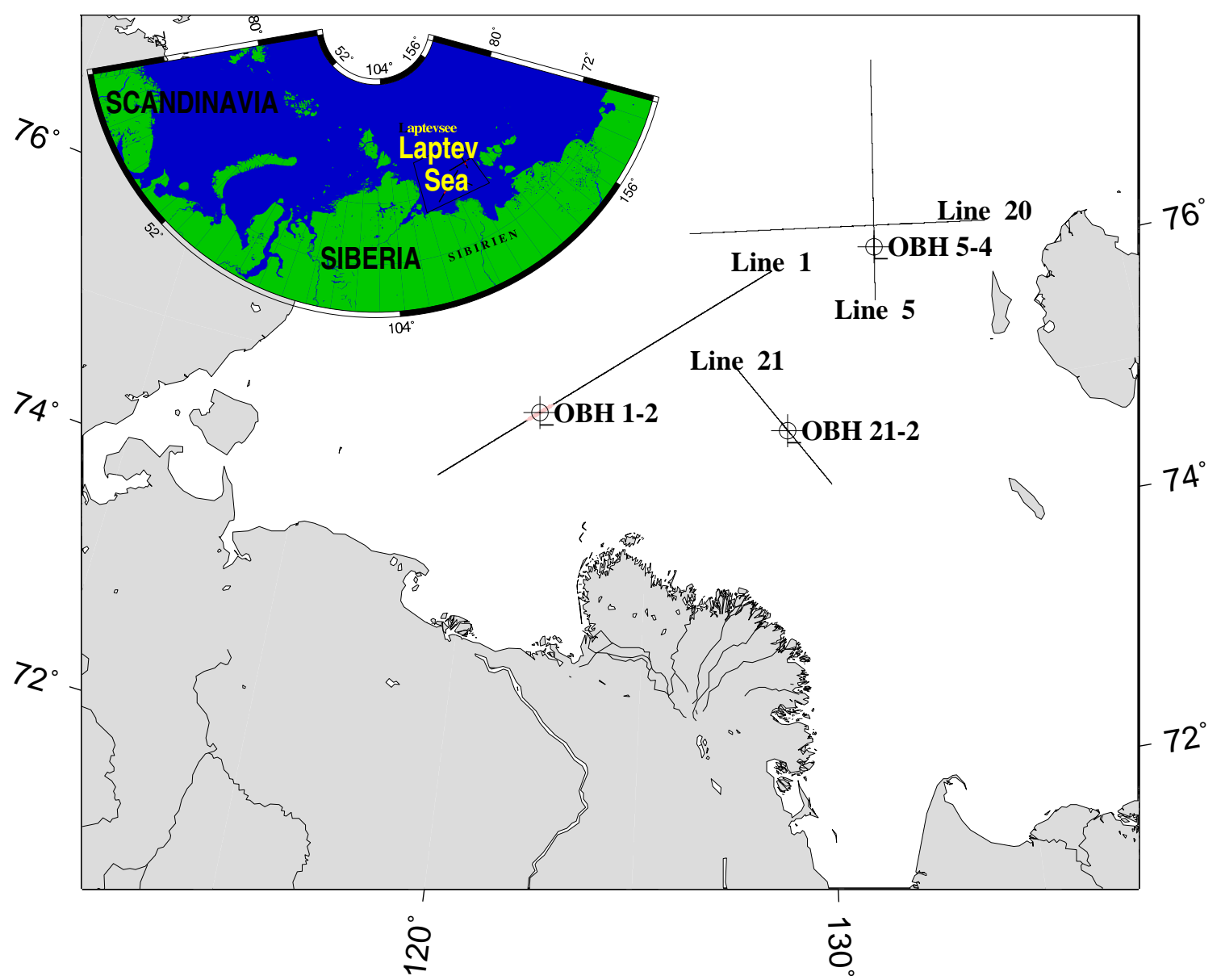

Figure 7.2: Location of study area and seismic lines. The data example of the multi-channel seismic lines have been selected near the locations of the OBH indicated in the map. 


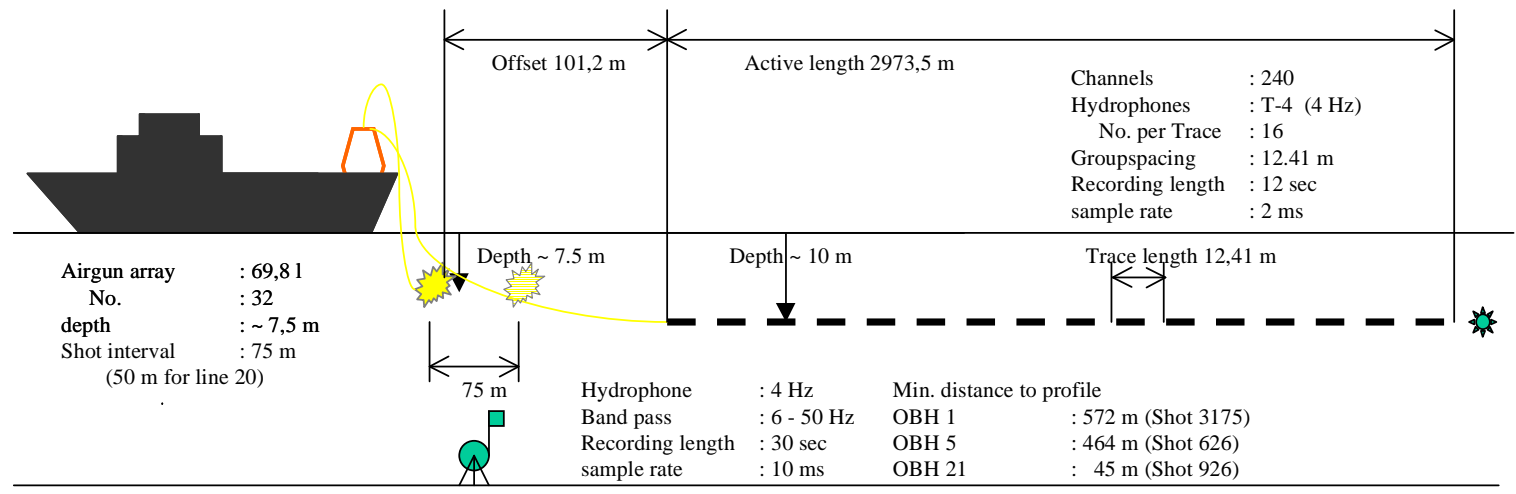

Figure 7.3: Acquisition parameter of the 1997 survey in the Laptev Sea conducted by the BGR and SMNG.

proposed by (Park et al., 1999), makes the above clearly visible, since three separated modes can be identified in the amplitude spectrum of the transformed wavefield. At high frequencies all modes tend to approach the same slowness of $\approx 0.7 \mathrm{~s} / \mathrm{m}$, equivalent to the velocity of $1430 \mathrm{~m} / \mathrm{s}$, the sound speed in the arctic cold waters of the Laptev Sea. At lower frequencies, however, the wave energy splits into distinct modes, which are characterized by the water depth (i.e. the thickness of the waveguide) as well as the sub bottom seismic parameters to be shown in this study.

\subsubsection{Regional Variation of Dispersion Curves}

The first indication that the dispersion characteristics is a function of lateral variability of the sediment structure is given by the variation of the dispersive pattern of the modes found on the different seismic lines. Fig. 7.7 shows the amplitude spectra of the transformed seismic wavefield from shot gathers of the other seismic lines located within the study area as shown in fig. 7.2. Here we can identify the variation in the position of dispersion curves with respect to the slowness-frequency axes, but also variations in the amplitudes between and along individual modes are present. Hence we assume, that the analysis of the full amplitude spectrum of the transformed wavefield will reveal more information on the sub bottom properties than conventional dispersion curve fitting methods, which are commonly used in seismology or geotechnical applications. 


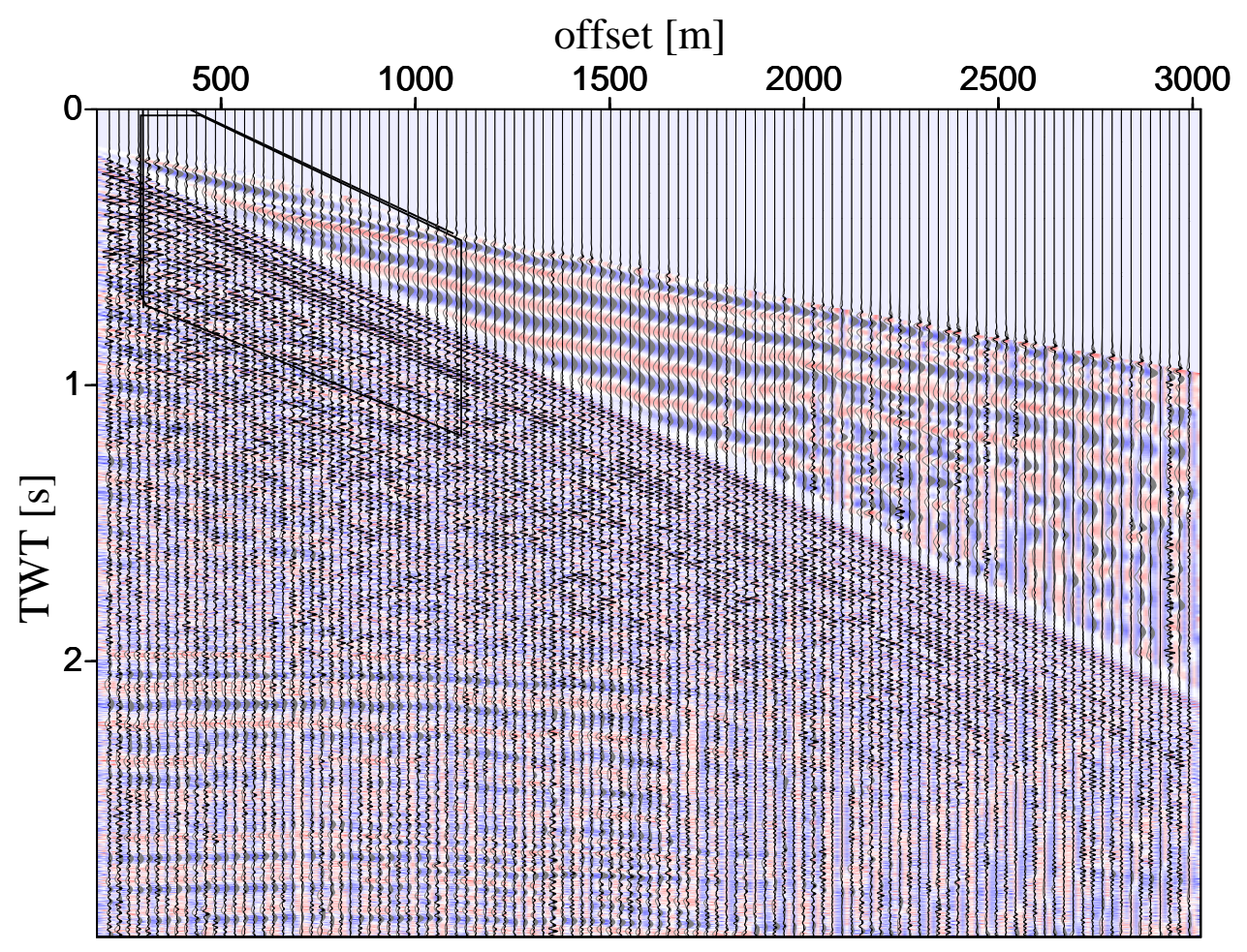

Figure 7.4: In the example shot gather from the reflection seismic line 21 the refracted arrivals with several reverberations have a velocity exceeding $3 \mathrm{~km} / \mathrm{s}$ related to shallow depth. This indicates the presence of submarine permafrost. The acoustic wave exhibits dispersion which is more clear in the time reduced zoom in fig. 7.5 and marked by the box. The amplitudes are scaled with AGC ( window $=0.1 \mathrm{~s}$ ). 


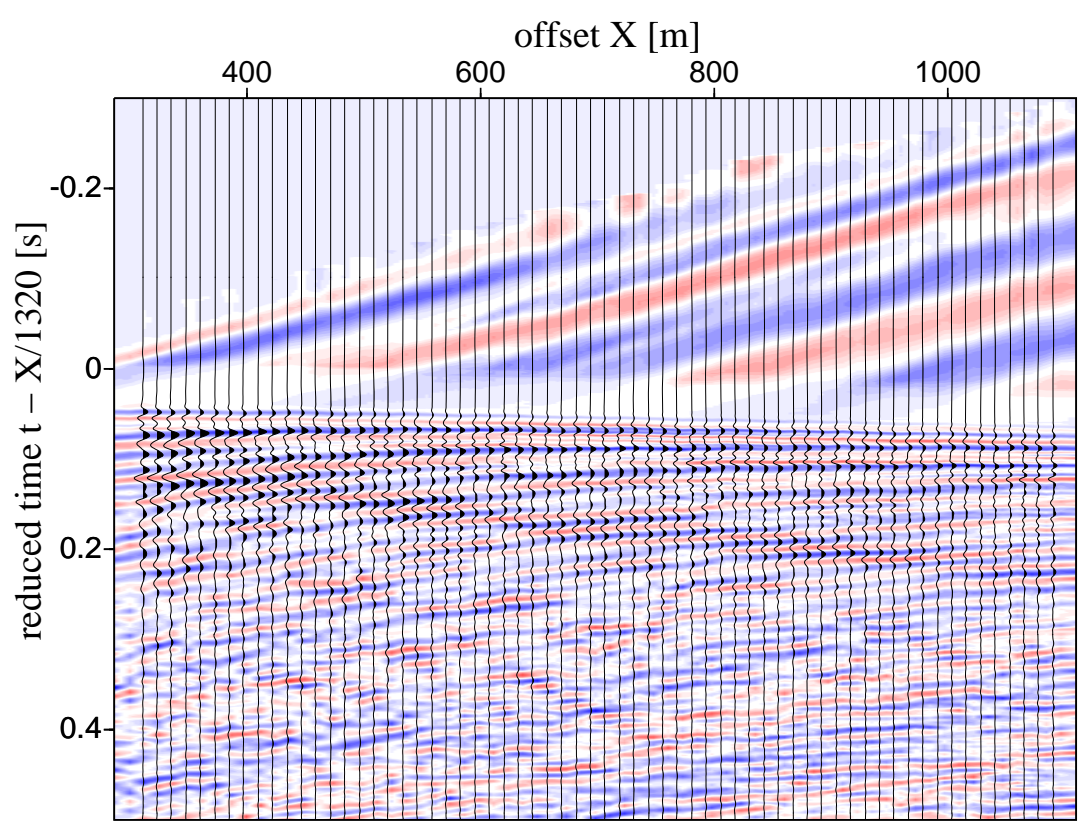

Figure 7.5: The time reduced section of the shot gather in fig. 7.4 with true amplitudes (offset dependend scaling for geometric spreading is applied for the wiggle traces. The colored backgroud image is scaled with AGC equivalent to the previous figure). 


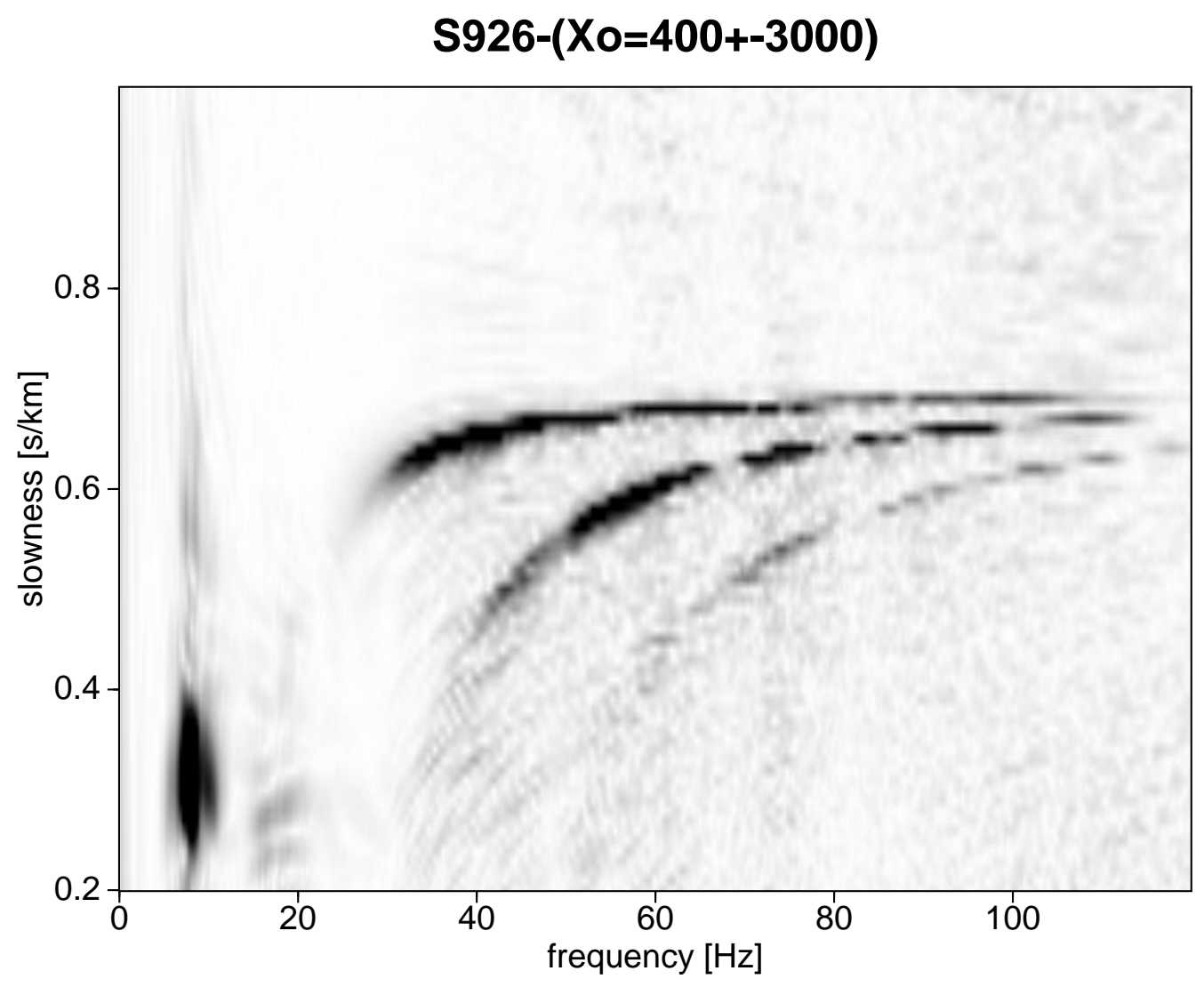

Figure 7.6: The spectral coefficients in the frequency-slowness domain have been obtained from successive $\tau-p$ and Fourier transforms of the seismic gather. The reflected and refracted energy is seen at $0.3 \mathrm{~s} / \mathrm{km}$ and $10 \mathrm{~Hz}$ while the three modes of the guided wave tend to the slowness of $0.7 \mathrm{~s} / \mathrm{km}$ corresponding to the acoustic velocity in the water. 

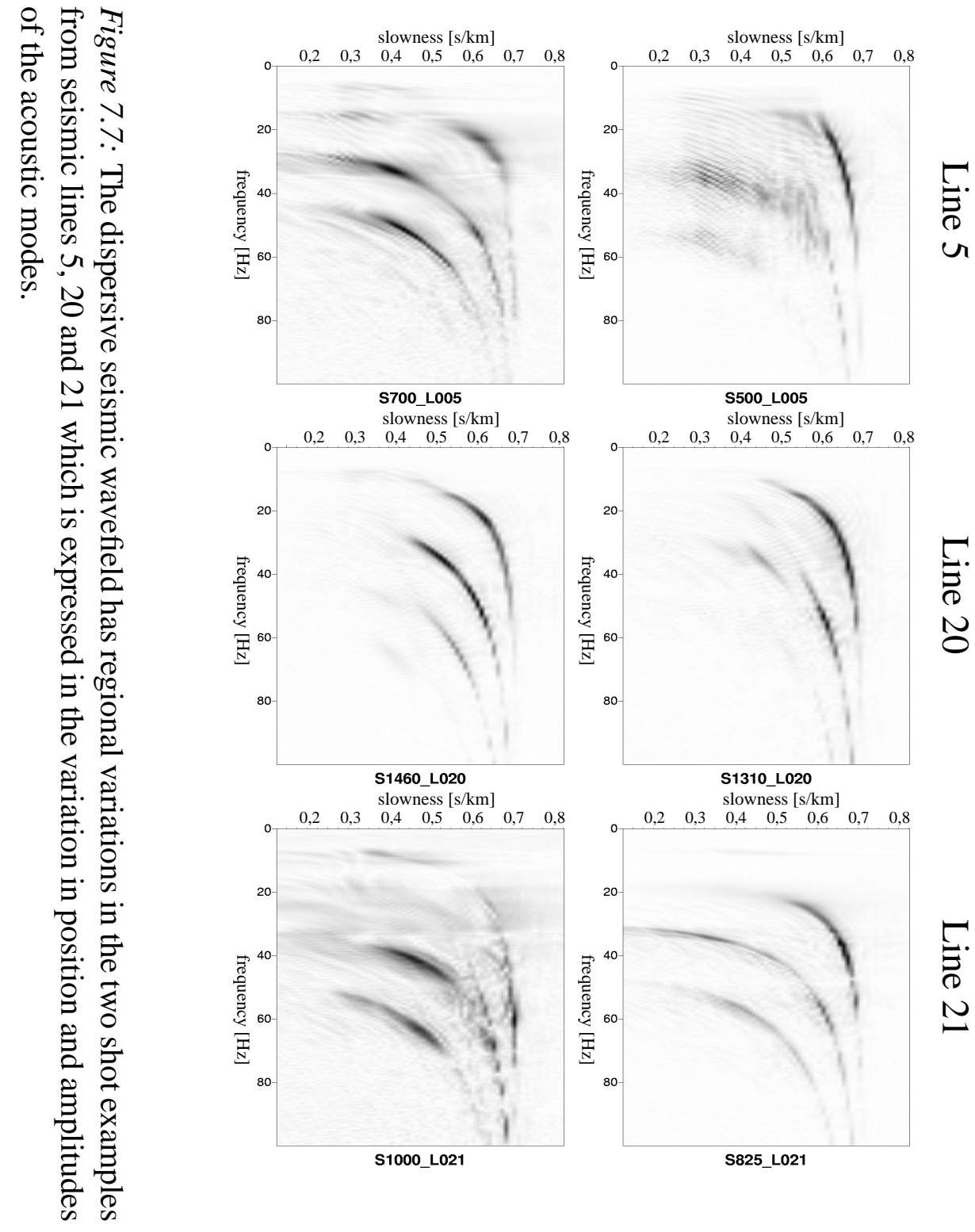

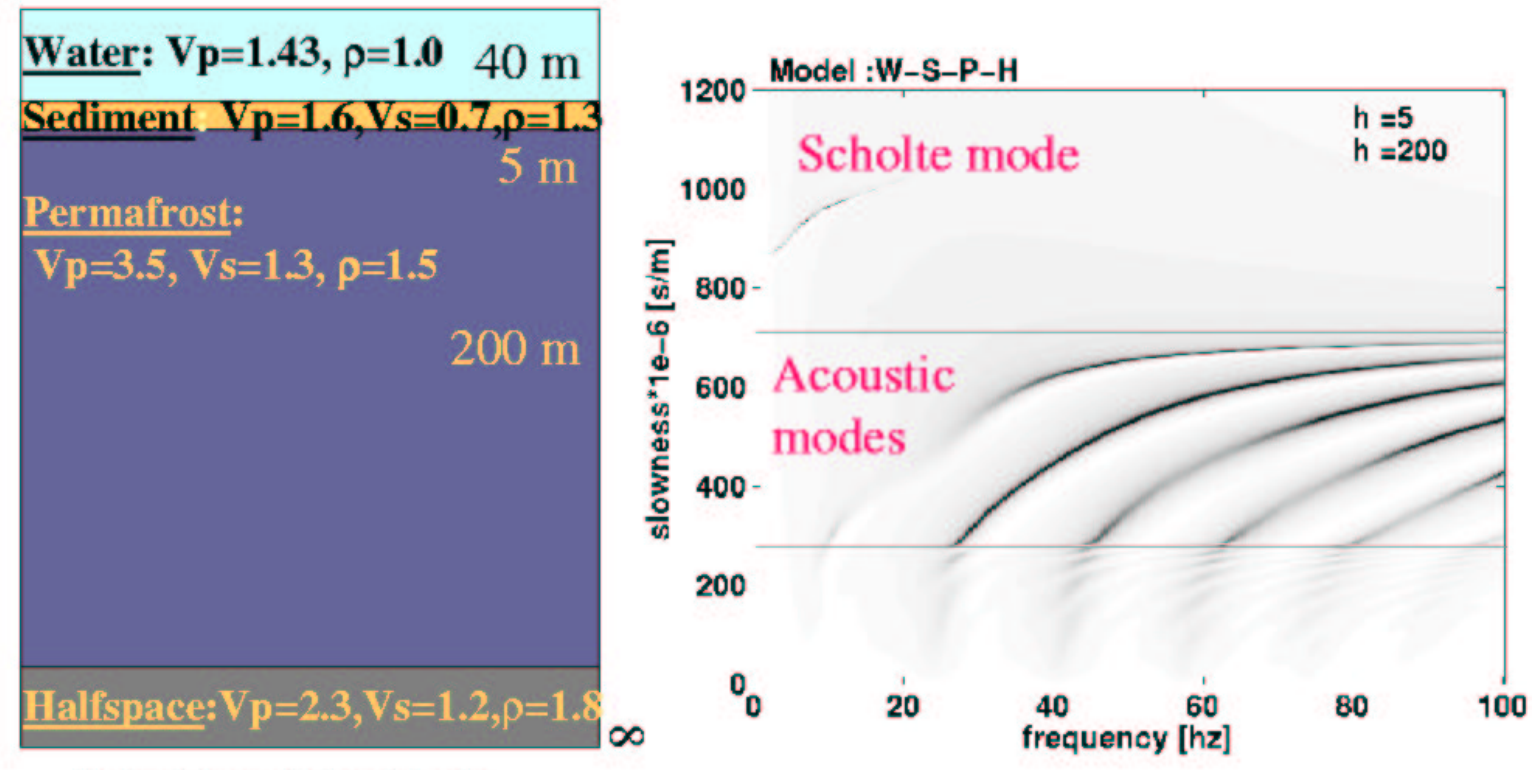

(velocities and densities in

$\mathrm{km} / \mathrm{s}$ and $\mathrm{g} / \mathrm{cm}^{3}$, respectively)

Figure 7.8: The reference model reflects the setting of a sub-marine permafrost overlain by a thawed layer fo sediments. The corresponding synthetic spectrum shows the acoustic guided modes in the slowness range corresponding to the limits from the water velocity and the permafrost compressional velocity.

\subsection{Sensitivity of Seismic Parameters of Dispersive Guided Waves}

In order to accomplish the analysis of the full amplitudes of the slowness spectrum of the transformed wavefield we need a method to predict the amplitude spectrum for known seismic parameters of the sub bottom. This is possible with forward modeling algorithm which solve the wave equation and therefore obtain solutions of the full wavefield including all body, boundary and leaky waves present. In the inversion process we will than be able to compare the measured data with the computed and revise the model until the best fit is reached, i.e. the misfit between the synthetic and measured amplitude spectra is minimized. 


\subsubsection{Reference Model}

The choice of a reference model to represent the main features of the Laptev Sea sediments is based on the results of a sensitivity study. This indicated a permafrost layer of several hundred meter thickness on top of the basement. The presence of layer of thawed sediments of a few meters thickness was significant to obtain a similar distribution of leaky mode energy as observed in the measured data. The water depth of $40 \mathrm{~m}$ is the average within the laptev sea study area. This model was used to show the dominant wavefield phenomena encountered in the study area and all variations of sediment parameters are based on this model. The corresponding synthetic slowness spectrum in figure 7.8(right) indicates that this reference model is qualitatively a good representation of the measured wavefield shown in figure 7.4.

Synthetic seismograms as well as time slices of the derivatives of the seismic potentials (div and curl) were calculated with a FD-algorithm (Bohlen, 1998), which enables to study the spatial distribution of the wave energy for each time step. The total wave energy is separated into the shear and compressional wave energy as displayed in fig. 7.9 for the seismic wavefield at 0.8 seconds after the excitation of the source at zero offset. The acoustic wave energy in the water column is visible in the offsets range from 1.2 to $1.5 \mathrm{~km}$ in the lower image. The polarity change just above the sea floor indicates the presence of a nodal plane and higher modes. Energy with exponential decay away from the fluid-solid interface is observed in the shear wave energy at $1 \mathrm{~km}$ offset and at $1.2 \mathrm{~km}$ in the compressional wave energy. The shear wave energy relates to the interface wave, since the arrival at 1000 $\mathrm{m}$ after $0.8 \mathrm{~s}$ corresponds to the Scholte wave velocity of the permafrost layer. The high amplitudes in the permafrost and sediment halfspace at offsets from 1 to $1.2 \mathrm{~km}$ as well as the exponential decaying energy in the sediment and permafrost layers at 1250 m must relate to the acoustic guided waves. Hence, we observe that energy related to the acoustic guided wave does penetrate into the sediment, and is therefore influenced by the sediment properties.

\subsubsection{Sensitivity of the Acoustic Guided Modes to Variations of Sedi- ment Properties}

The sensitivity of the acoustic guided modes was investigated with two methods. The computation of sensitivity kernels quantifies the sensitivity of each individual normal mode of the seismic wavefield. The variation of the sensitivity kernel with depth indicates the most significant regions for each mode. This enables to investigate the variation of sub-surface properties at specific depth for the individual normal modes.

However, with seismic experiments we observe the superposition of all modes. The influence of variations of one parameter on the wavefield can be visualized from the partial derivatives of the inversion misfit with respect to the model parameters obtained in the inversion process. Furthermore, the variation of the full seismic spectrum in the $p$ - $f$ domain 

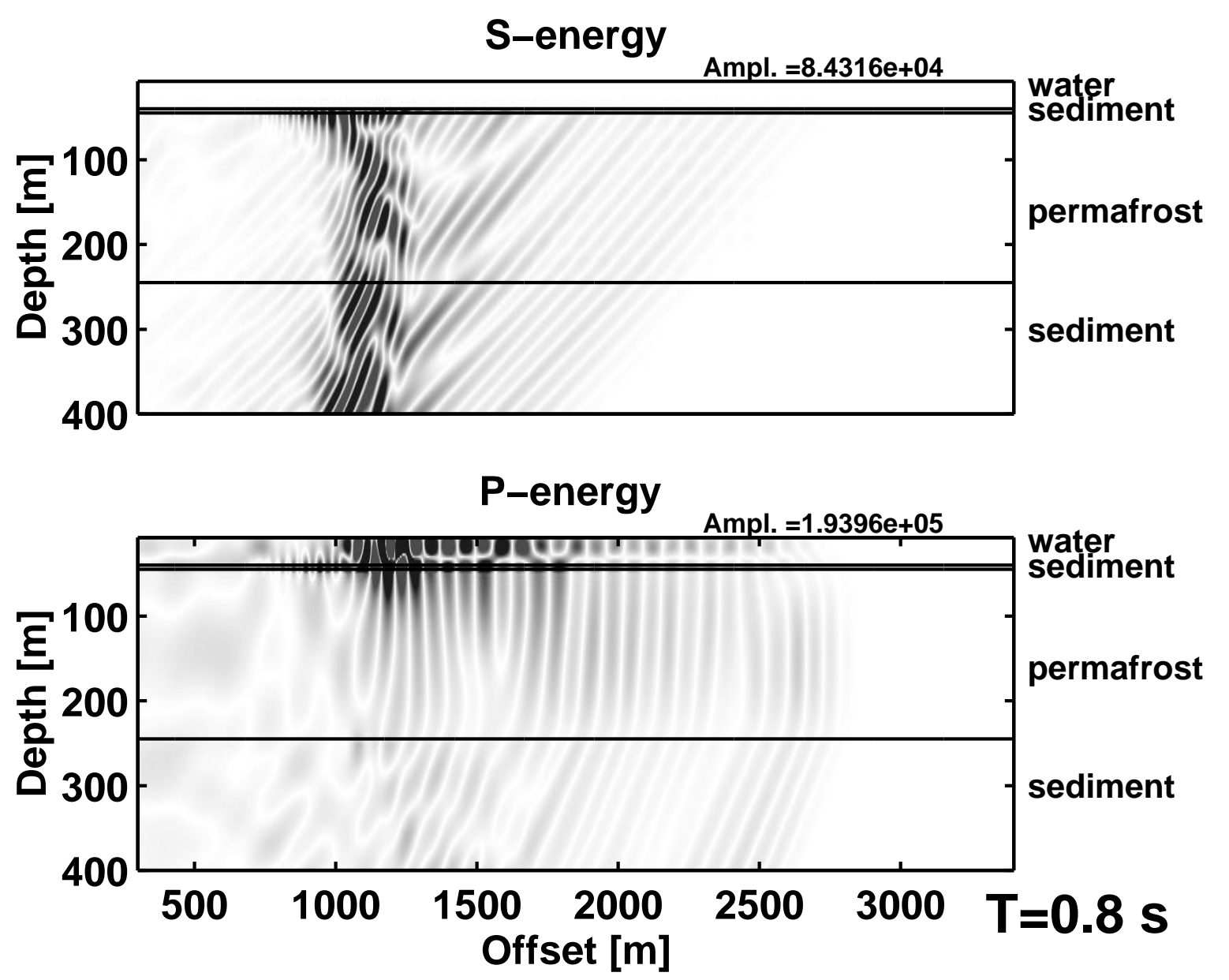

Figure 7.9: Snapshot of the shear (top) and compressional (bottom) wave energy of the synthetic seismic wavefield at 0.8 seconds after exciting an explosive source at zero offset computed with the FD-algorithm (see text for desctiption of arrivals). 


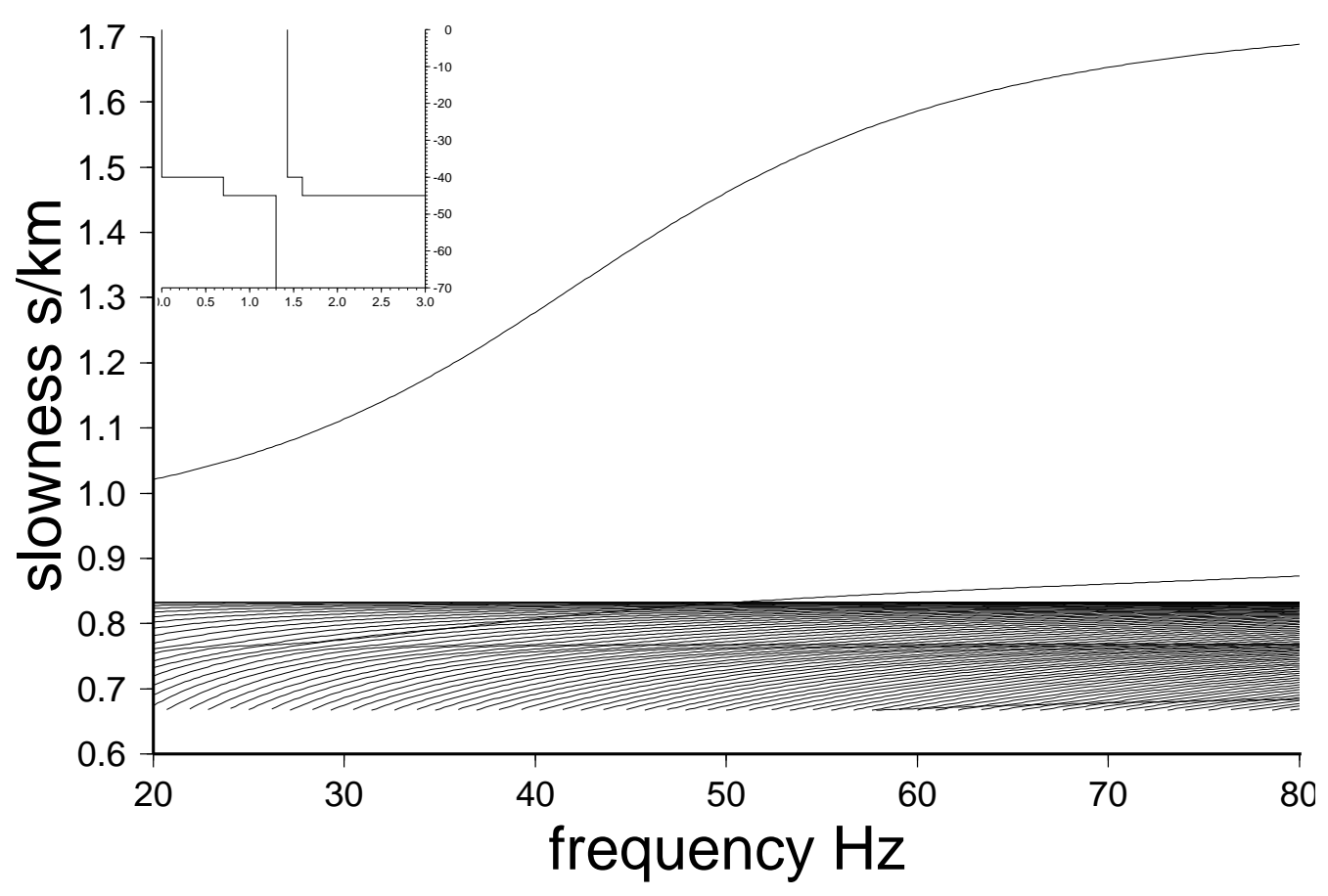

Figure 7.10: The dispersion curves obtained from the normal mode solution for the reference model shows that the number of normal modes is much higher than the observed modes.

can be investigated by forward modeling. A sequence of images of the spectral coefficients is obtained from models with stepwise varying parameters. The animation of these images visualizes the amplitude changes of the dispersive modes. This approach is a more qualitative investigation of the sensitivity to parameter variations.

The dispersion curves of the fundamental mode visible at slowness values exceeding 0.85 $\mathrm{s} / \mathrm{km}$ and numerous higher modes are visualized in figure 7.10. The use of normal mode computations for dispersion curve interpretation of the measured data is nearly impossible.

The eigenfunctions of the vertical and horizontal displacement (top) and the sensitivity kernels for variations of the shear wave velocities for the fundamental mode are shown in figure 7.11. These can be used to calculate the energy distribution with depth shown in 7.12 for the fundamental and the first higher mode starting at about $50 \mathrm{~Hz}$. At a fixed frequency, one observes deeper penetration of the first higher mode compared to the fundamental mode. The amplitude of the kernel for the fundamental mode is much higher than for the first higher mode indicating that the influence of shear wave velocity variations on the fundamental mode is higher. This method to visualize the energy distribution helps to further the understanding on propagating modes. 

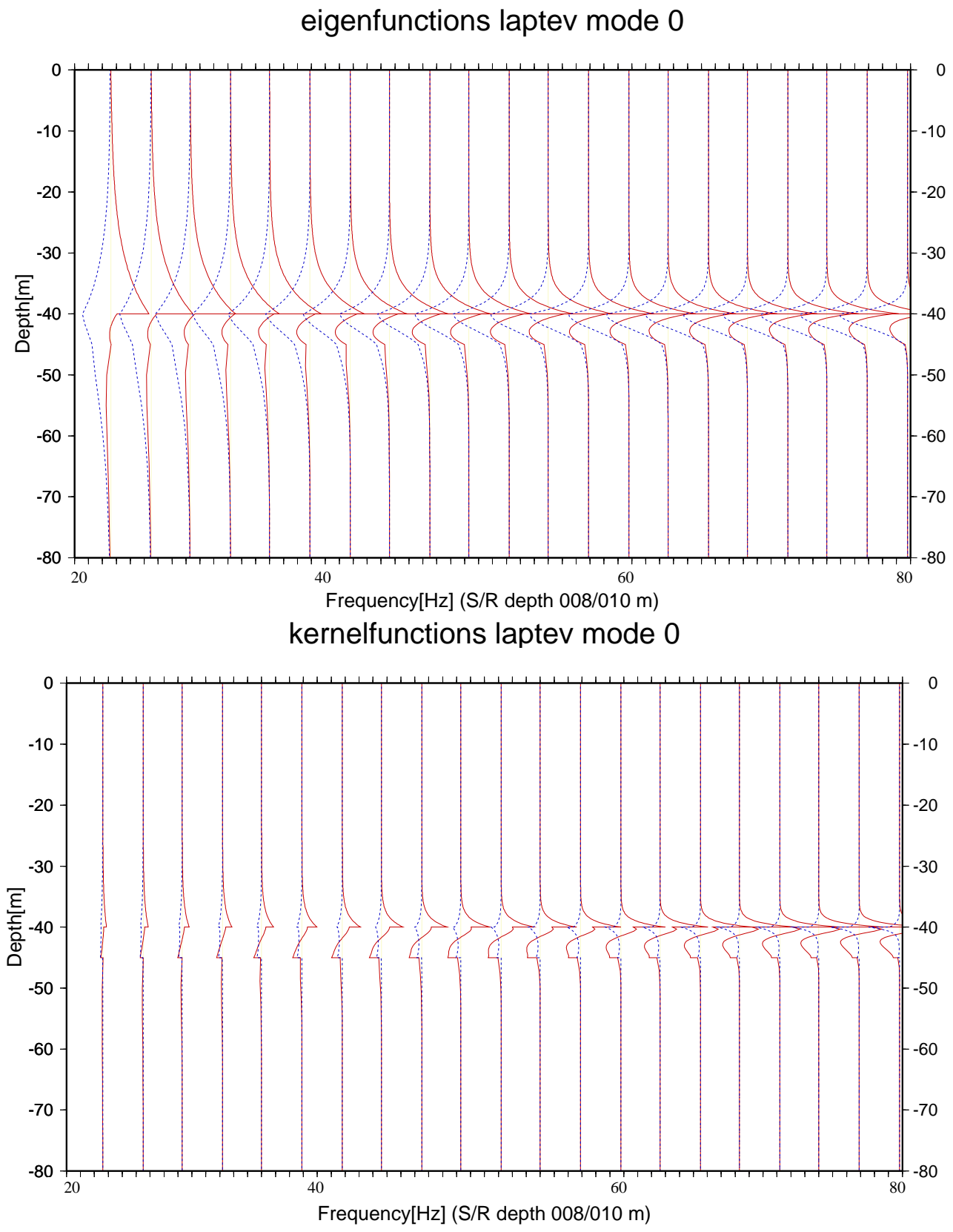

Figure 7.11: Depth variation of the eigenfunctions (top) of horizontal and vertical diplacement and the corresponding sensitivity kernels with respect to shear wave veolcity indicate the penetration depth as a function of frequency. 


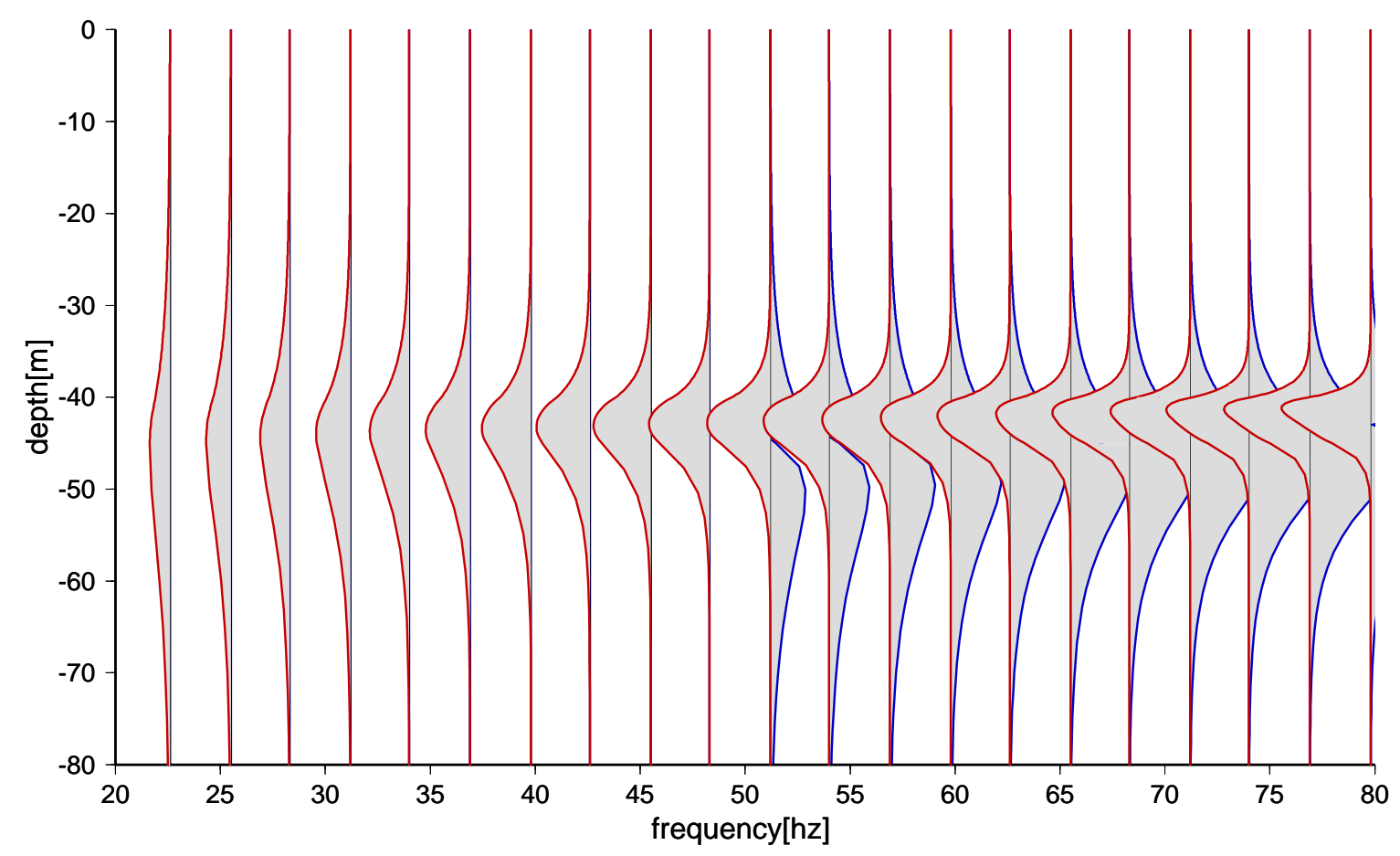

Figure 7.12: The comparison of the energy variation with depth for the fundamental (red) and first higher (blue) mode shows the deeper penetration for the higher mode at high frequencies.

Next, the partial derivatives for variations in compressional wave velocity and density are investigated (Fig. 7.13). The sensitivity to shear wave velocity varitions in the sediment is one order of magnitude higher than the other parameters, but ist should also be noted that the influence of the parameter variations affects different modes. E.g. the third higher mode is affected by compressional wave variations, while the second higher mode as well as the fundamental mode is influenced by all parameters shown. This varies significantly for different models and intermediate models obtained within an inversion run can be very complex. However, in it is obvious that compressional wave velocity and to a certain extend also the density of the model must be considered as an inversion parameter or given accurately from other a priori information.

The forward modeling of seismic wave spectra was performed for variations of one parameter with respect to the reference model of fig. 7.8.

The variation of the permafrost thickness affects the energy distribution of the leaky modes at slowness values lower than the corresponding seismic velocities of the halfspace. Very thin modes with little energy can be identified in the synthetic wave spectra, if the per- 

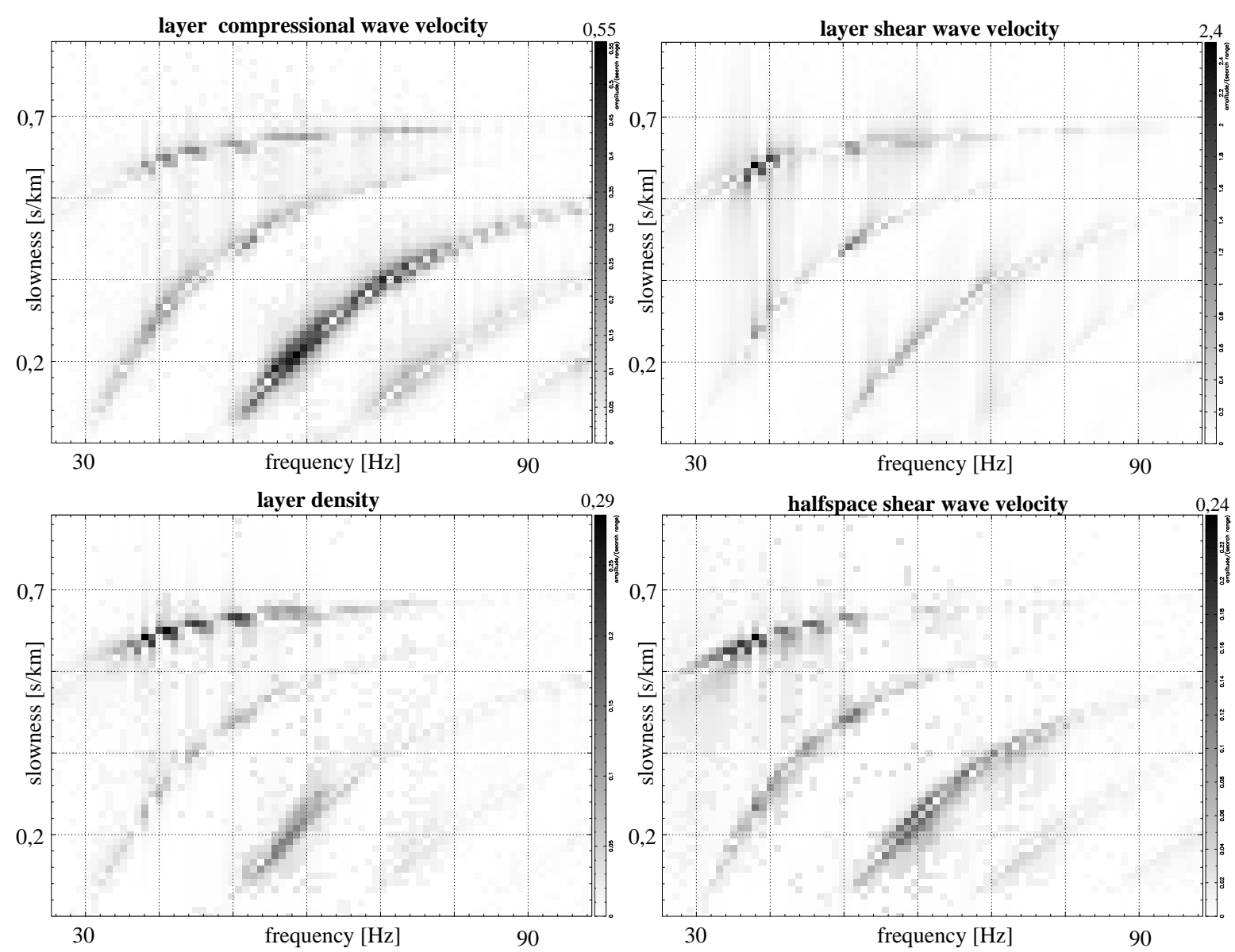

Figure 7.13: From the partial derivatives of the misfit function with respect to the model parameter of the final inversion model the sensitivity of individual modes to a certain model parameter can be investigated. The sensitivity to variations in Vp and Vs (top) and the density (bottom) of the sediment layer as well as Vs in the permafrost (bottom right) are plotted. Note the different absolute amplitudes of the scale $(\mathrm{A}(\alpha)=0.55, \mathrm{~A}(\beta)=2.4, \mathrm{~A}(\rho)=0.29$, $\left.\mathrm{A}\left(\beta_{\text {perm }}\right)=0.24\right)$. 

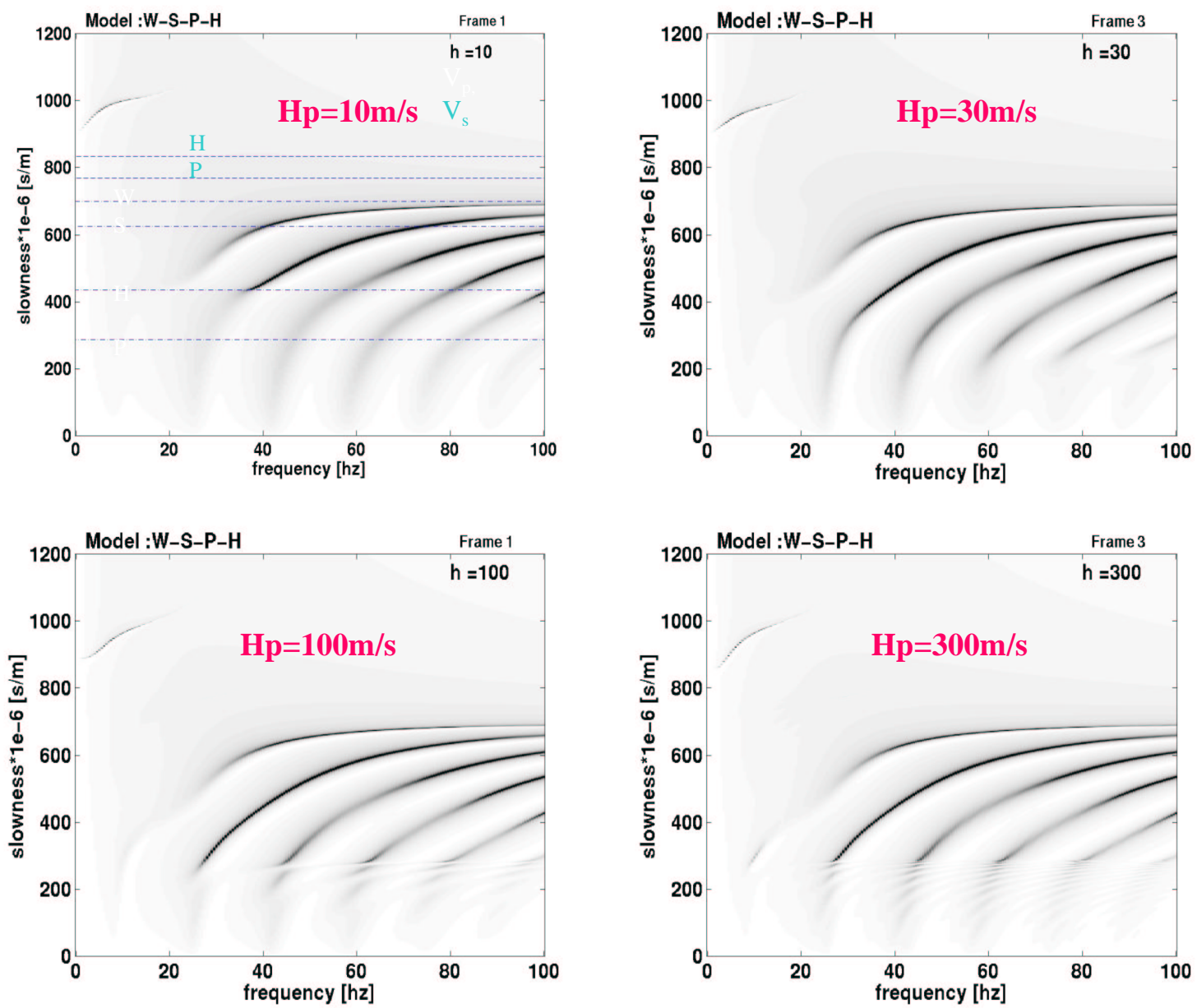

Figure 7.14: The presence of a thick permafrost layer $(>100 \mathrm{~m})$ gives rise to additional modes below $250 \mathrm{~s} / \mathrm{km}$ with increasing number for a thicker layer. The leaky mode energy for the $30 \mathrm{~m}$ layer (top right) is in smooth transition to the normal modes. For the other images a more sudden change in amplitudes is observed at the slowness corresponding to the $\mathrm{p}$-wave velocity of the permafrost layer. 
mafrost layer is several hundred meter thick (Fig. 7.14). Similar patterns have been observed in some of the measured wave spectra of the seismic line 5. However, these features are not significantly above the noise level.

The variation of the shallow sediment properties affects the amplitudes of the spectral coefficients at much higher level. The variation of the shear wave velocity of the sediment affects most dominantly the interface waves, but interference patterns between higher mode interface waves and acoustic guided waves can be seen in some of the synthetic spectra shown in figure 7.15.

The variation of the thickness of the sediment layer in the range from 1-15 $\mathrm{m}$ is presented in figure 7.16. The amplitudes of the acoustic modes vary dramatically. The amplitudes of the higher modes of the guided wavefield is dramatically enhanced, if the sediment layer exceeds a few meters of thickness. Additional nodes and complex mode interference occurs with increasing thickness.

The sensitivity study shows that the variation of the sediment layer properties has strong influence on the complexity of the dispersive modes, specially their amplitude distribution. Hence a sophisticated inversion scheme taking the amplitudes of the full wavefield into account can provide more detailed information compared to conventional inversion methods regarding the dispersion curve of the fundamental mode only.

\subsection{Inversion}

\subsubsection{Inferring Seismic Parameters from Shot Gathers in two Stages}

The derivation of the variation of shear wave velocity with depth is based on the analysis of the dispersion of the seismic interface waves, i.e. the frequency dependent variation of the velocity of propagation of the seismic wave energy. Assuming that the marine sediment can be described as an laterally homogeneous medium, we can describe the propagation of seismic energy within the medium by the elastic wave equations. The solution to the wave equation defines the dispersion relation for a assumed distribution of seismic parameters. The dispersion characteristics can therefore be obtained by a search of the diminishing determinant of the wave equation. Alternatively the full solution of the wave equation in form of the slowness spectrum reveals the frequency dependence of the propagation velocity. The solution of the forward problem has been applied to the marine environment, e.g. by Wang (Wang, 1999). Using this propagator matrix method to calculate the full seismic wavefield in the slowness frequency domain is very useful and effective, since the computationally expensive task to transform the wavefield into the distance-time(x-t)-domain representation as seismograms of the wavefield can be omitted. Secondly, the transformation into the x-t-domain involves the convolution with oscillatory function, hence the description of the wavefield in 

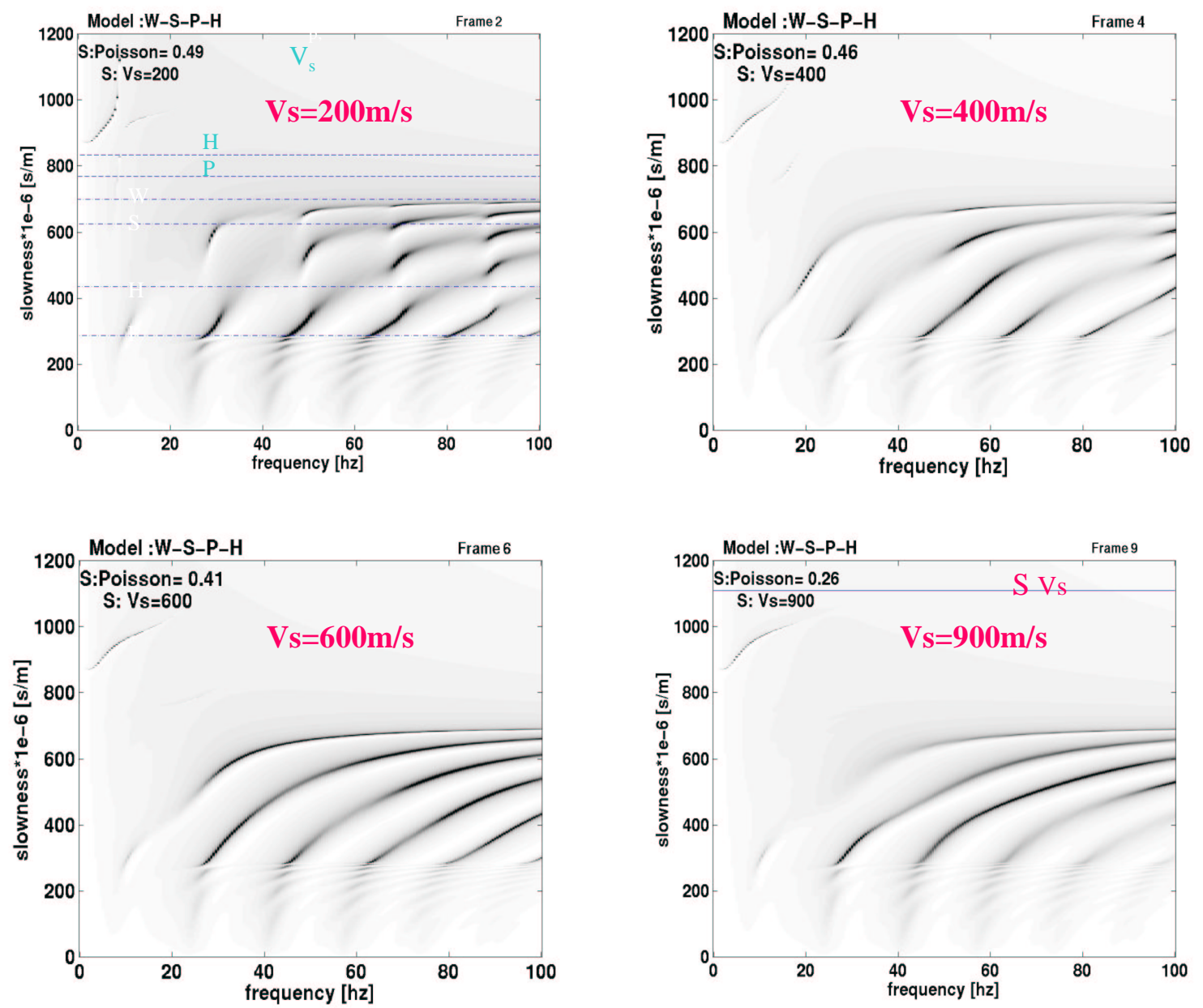

Figure 7.15: The variation of shear wave velocity affects the dispersion pattern most for low shear wave velocites. The amplitudes of the acoustic modes are restricted to small patches. This becomes less severe as the Poisson number tends to $1 / 4$, i.e. the sediment corresponds to the normal compressional to shear wave velocity ratio of $\sqrt{3}$ commonly used for consollidated sediments and rocks. 

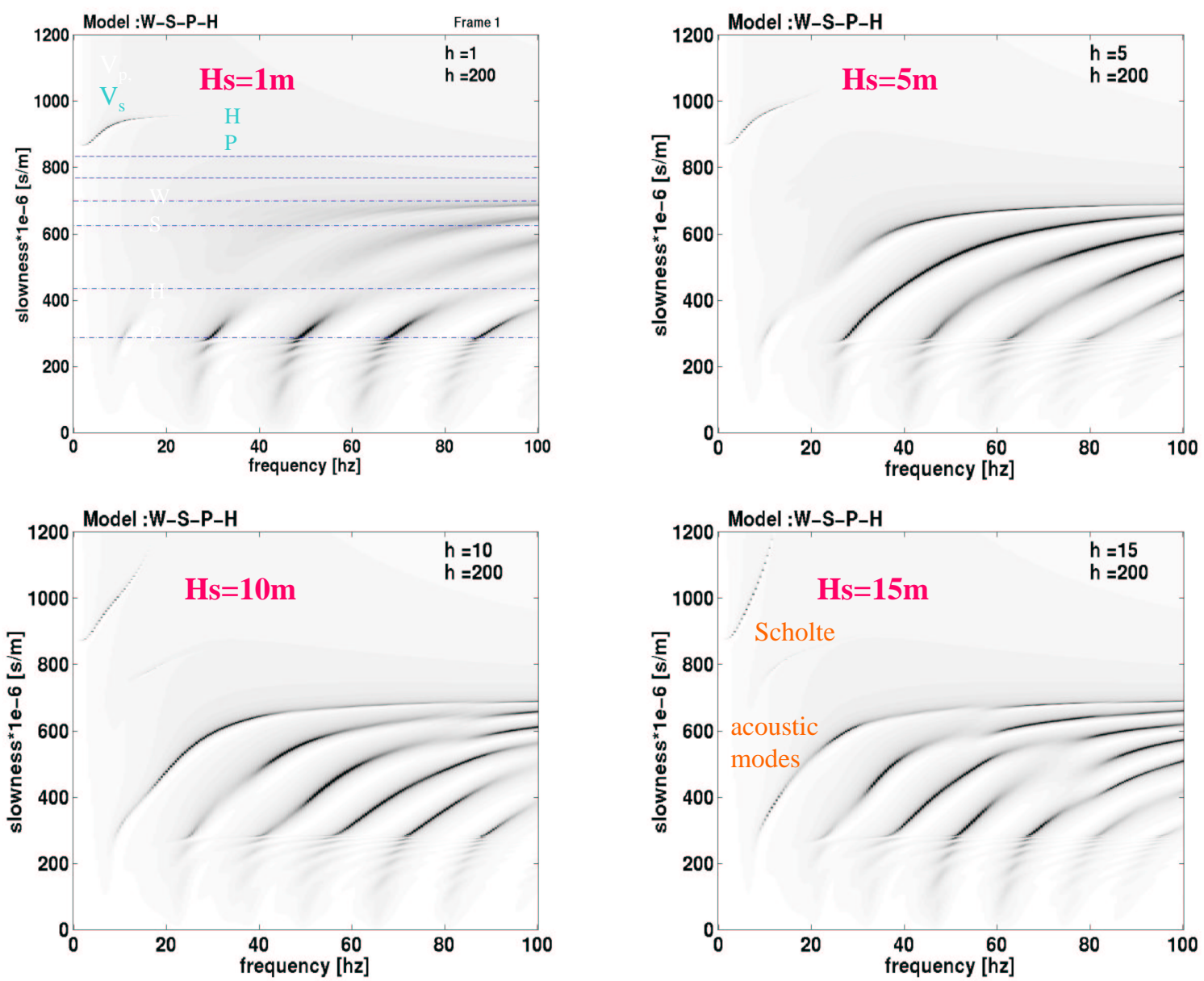

Figure 7.16: The variation of the sediment layer thickness influences the amplitude distribution of the acoustic modes significantly. A thick sediment layer causes interference patterns and discontinous acoustic modes while a thin layer bounds most energy to the permafrost layer velocity. The 5 - $10 \mathrm{~m}$ thick layer has the smoothest and highest amplitudes of acoustic guided modes. 
the time domain is highly non-linear.

The dispersion relation is depending merely on the sediment properties, yet the dependence is not resolvable analytically. Since the computation of dispersive wavefields from seismic parameters is feasible (e.g. (Wang, 1999)), the derivation of the seismic properties from the measured dispersion can be achieved by means of inversion procedures to the forward problem. The inversion method proposed is a full wavefield inversion, which is independent of the identification of distinct dispersive modes. It was developed by Forbriger for the inversion of shallow-seismic Rayleigh-waves (Forbriger, 2003a; Forbriger, 2003b) and extended to be used for shallow marine applications.

The inversion procedures avoid to deal with dispersion in terms of normal modes and has the potential to exploit true amplitude information of the full wavefield including all fundamental, higher and leaky modes. This additional information yielded in the amplitude distribution of dispersive modes is neglected in methods based on matching the dispersion curve pattern, which are more easily applied to the data.

In order to make the algorithm of Forbriger applicable to marine seismic data, some adaptions needed to be applied. The forward modeling algorithm published by Wang was used in the inversion process (Wang, 1999). Additional modifications had to be applied to account for near field effects in the water column, which are generally not recorded in seismic data, but dominate the theoretically calculated wavefield in the close proximity of the source.

The inversion procedures applied to the dispersive seismic data can be divided into two stages. The first is the wavefield transform of the measured data into the slowness-frequency domain. The second stage is the derivation of sub-surface properties by means of an iterative inversion procedure (see chapter 5). The input data set (Fig. 7.4) was tapered and scaled prior to the application of the modified Fourier-Bessel transform. Corresponding to the 12.4 $m$ group spacing of the streamer the spatial aliasing is not in the frequency range up to $90 \mathrm{~Hz}$ for the acoustic modes below $0.8 \mathrm{~s} / \mathrm{km}$ slowness.

\subsubsection{Inversion Results}

The inversion of the spectral coefficients was started with a travel time inversion of first arrival picks beginning at the reference model with fixed shear wave velocity and density values. Then the inversion of of the spectral coefficients with respect to shear wave velocity was performed. The velocity-depth models of the traveltime inversion and the inversion of the spectral coefficients are compared in fig. 7.17. The poor results of the traveltime inversion at shallow depth is mainly due to the lack of near offset traces.

The spectral coefficients of the intermediate result (Fig. 7.18, top) of the travel time inversion compared with the final result (Fig. 7.18, bottom) shows the improvement obtained by the inversion of the wavefield spectrum. The saturation of the colors corresponds to the amplitude of the complex coefficient, while the phase is color coded. The corresponding 

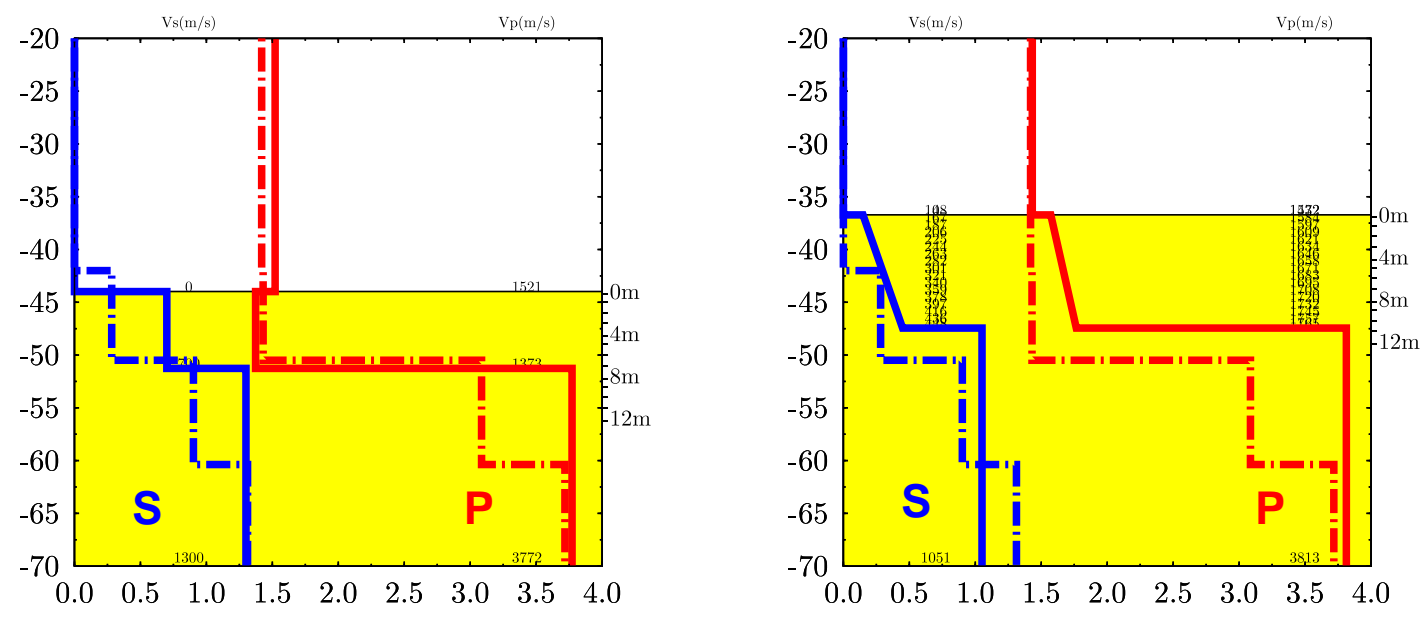

Figure 7.17: The comparison of the intermediate and final models (right) indicates the variability of the results if only limited a priori knowledge is used. The water depth changed significantly from $44 \mathrm{~m}$ to $34 \mathrm{~m}$.

seismic velocities of the model are plotted in fig. 7.17. Including a low velocity zone in the travel time model did enhance the fit of the model significantly. First inversion runs had to be applied with normalization of the data set. But, the data fit could be significantly improved once the near-field term of the forward algorithm was identified and corrected for (c.f. section 5.4). The introduction of the first and second polynomial order of the seismic velocities enhanced the fit significantly and there was no more need for a low velocity zone in the data. This evolution of inversion results indicate, that interpretations of the inversion results have to be treated with care. The selection of the final model, hence the model with the best fit to the measured data, is therefore subject to the choice of weighting of the a priori knowledge and the constraints.

\subsubsection{Resolution Analysis}

A resolution analysis with the final model shows the trade-off between parameters as well as the parameter variations obtained for a misfit tolerance of $10 \%$. The resolution analysis is performed as a "rubber band test" by a sequence of inversion runs. Each of the free inversion parameters is fixed at levels $10 \%$ higher and lower than the parameter value in the final model, while the other free parameters are inverted by consecutive runs. The set of models obtained 

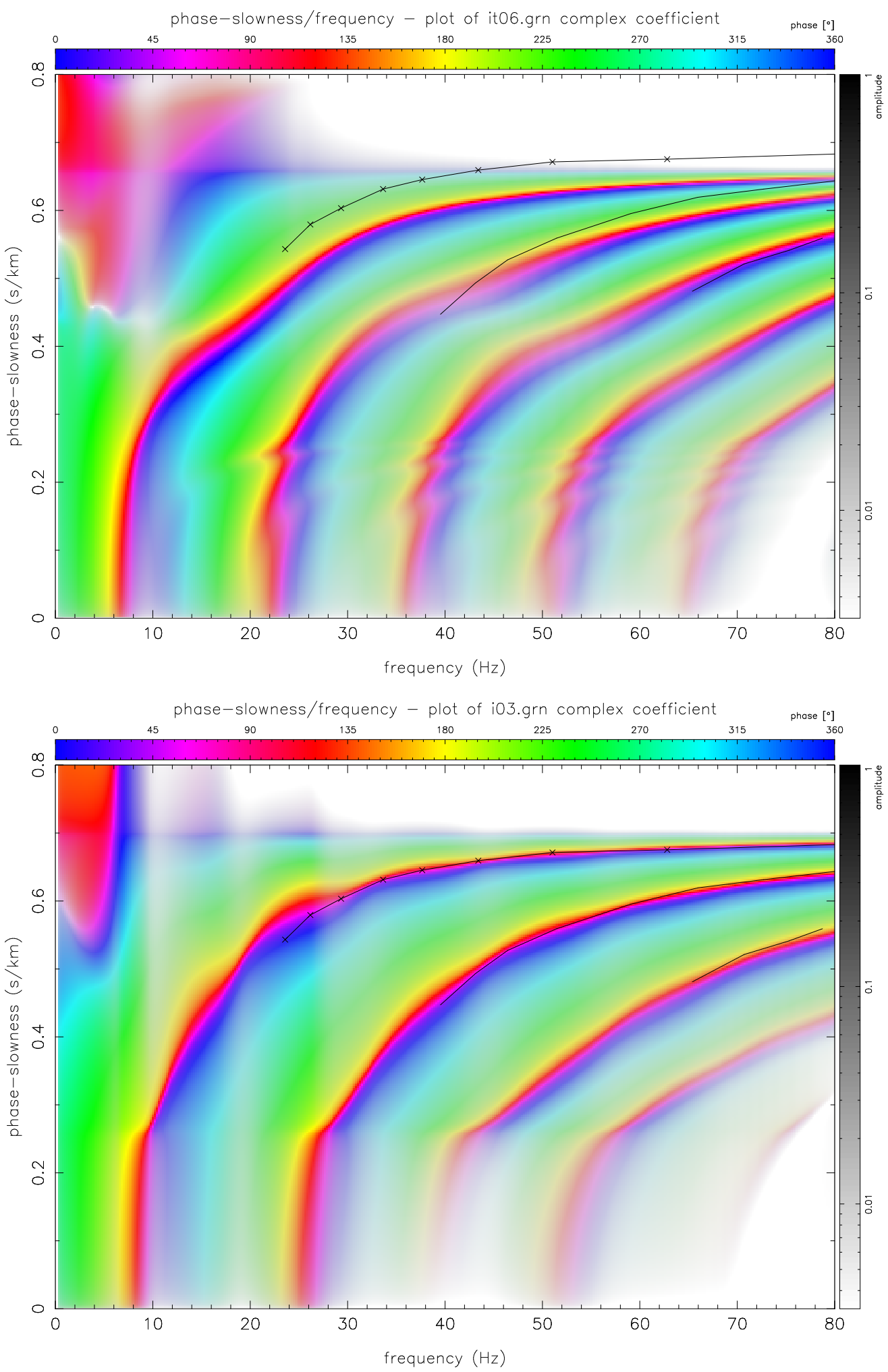

Figure 7.18: The wavefield spectrum of the intermediate model (top) obtained from the travel time inversion does not fit the measured wavefield spectrum indicated by the solid lines. The synthetic wavefield spectrum of the final model (bottom) gives an acceptable fit. 


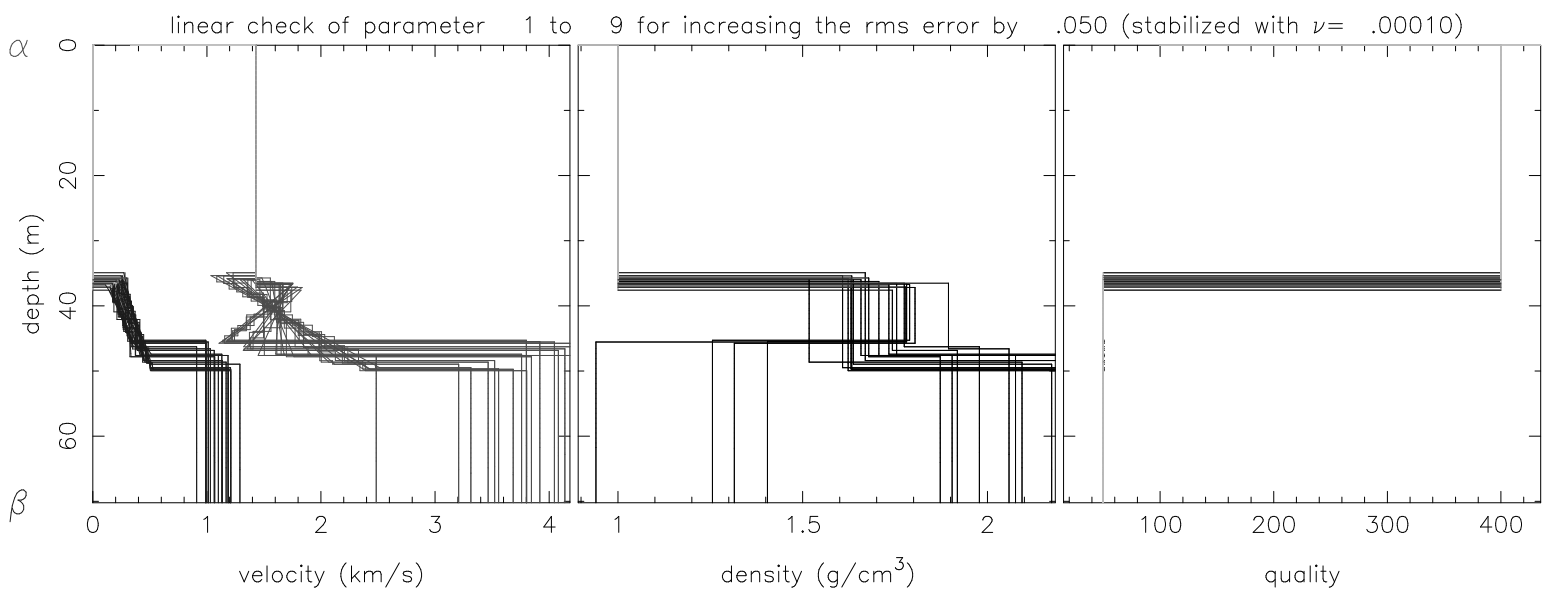

Figure 7.19: Resolution analysis for the final inversion model.

for the variations of one parameter can be used to visualize and identify trade-off parameters. The sets of models of the sequence defines the range of variations of all the free parameters.

The resolution analysis presented in figure 7.19 shows variations of all free parameters including shear and compressional wave velocities and density of all layers. The shear wave velocity profile is much better determined than than compressional wave velocities with a resolution by $\approx 20 \%$. The density in the sediment layer is not very sensitive while the density of the lowest layer is not determined at all. The trade-off is more easily checked if only selected parameters are plotted at the same time. The resolution analysis only for the shear wave velocity in the halfspace $\beta_{H}$ is compared to the density in the sediment layer. It is remarkable that variations in $\beta_{H}$ cause a variation in the density, but not the other way.

\subsection{Conclusions}

The dispersive seismic wavefield of acoustic guided waves has been observed with long offset streamer measurements. The investigation of this wave type has shown that this wave type is sensitive to compressional and shear wave velocity of the shallow sediment structure. Most dominant parameters are the layer thicknesses, but seismic velocities can be resolved in the first few tens of meters.

The presence of a thin layer $(5-10 \mathrm{~m})$ of thawed sediments is confirmed with the inversion results obtained from the analysis of the measured acoustic guided waves. This correlates with independent results from sediment coring in this area (Franke et al., 2001). 

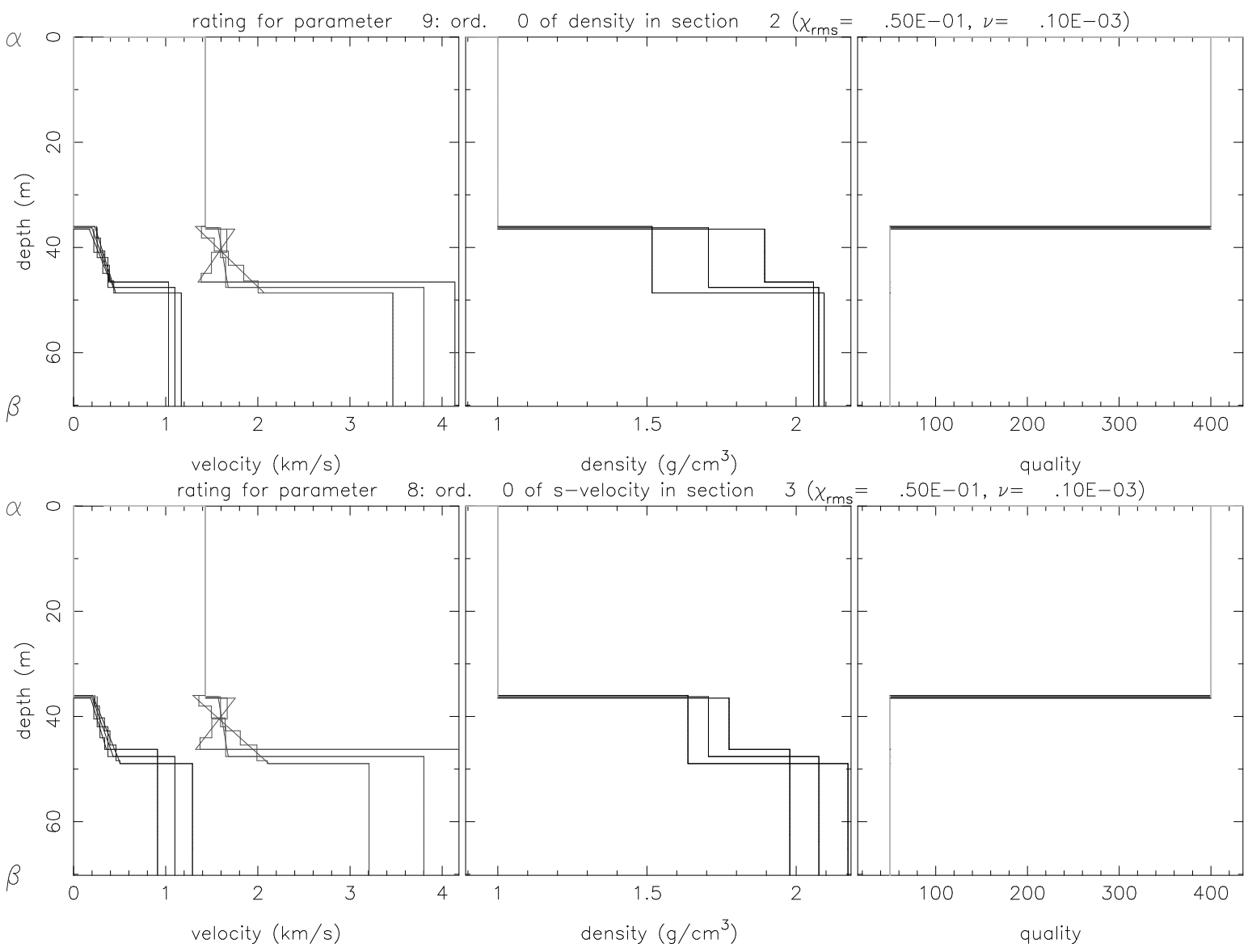

Figure 7.20: The resolution analysis of the shear wave velocity in the permafrost compared with the density of the sediment layer indicate the trade-off between these two parameters. Remarkably is that the trade-off for variations in Vs to the sediment density is stronger than vice versa. 


\section{Chapter 8}

\section{Dispersive Wave Studies in the Arkona Basin Natural Laboratory, Baltic Sea}

\section{Contents}

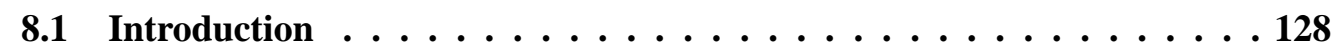

8.2 The Arkona Basin Study Area $\ldots \ldots \ldots$

8.3 A Towed Data Acquisition System $\ldots \ldots \ldots$. . . . . . . . . . . . . . . . . . . . . . . .

8.4 Acquisition Tests . . . . . . . . . . . . . . 132

8.4 .1 Towing Depth . . . . . . . . . . . . . . . . 132

8.4 .2 Offset Range . . . . . . . . . . . . . . . . . . . . 135

8.4 .3 Data Sets . . . . . . . . . . . . . . . . 135

8.5 Determining Sediment Stratigraphy and Velocity Models . . . . . . . 138

8.6 Acquisition and Preprocessing of the OBS-Data $\ldots \ldots \ldots$

8.7 Analysis of Dispersive Wavefields . . . . . . . . . . . . . . . . 147

8.8 Inferring the Shear Wave Velocity by Inversion of the Wavefield Spectra 152

8.9 Conclusions . . . . . . . . . . . . . . . . 158 


\subsection{Introduction}

The further developing of acquisition methods towards a towed acquisition system and the comparison of first analysis results are the topics presented in this case study. These were subjects of the investigations of the INGGAS-NATLAB subproject. Investigations to use a towed acquisition system to obtain dispersive seismic data included the practical considerations of operating a deep-towed streamer at a determinable depth and estimate the influence of the receiver configuration on the analysis methods. Secondly, the analysis of the measurements of dispersive waves from two OBS stations is compared to the deep-tow streamer recordings as well as to sediment physical data available for the study area.

In order to accomplish these tasks, a study area was selected, where comprehensive information on the sediment physical properties is available or easily obtained, and which is suitable for the tow-experiments for the acquisition method to be applied. The Arkona Basin in the Baltic Sea has been devoted a Natural Laboratory (NATLAB) for this purpose, since the area has been studied in numerous previous investigations providing stratigraphic and sedimentologic information as well as experience from previous seismic investigations in the area (Moros et al., 2002; Lemke, 1998; Endler, 1989).

Previous seismic investigations in the study area were performed within the framework of the EU funded MAST project and included investigations with the Geophysical In-Situ Probe (GISP) penetration sonde. The measurements of in-situ parameters of the sediments including density and seismic velocities were presented in several reports and publications (Ayres \& Theilen, 1999; Lange, 1997; Theilen et al., 1993; Theilen \& Pecher, 1991). Further high-resolution seismic profiles are available form various cruises in this area.

\subsection{The Arkona Basin Study Area}

The Arkona Basin is situated in the transition zone between the Baltic Shield in the North and the North-German-Polish-Trough in the South. It is marked by the Tornquist Fracture Zone striking in NW-SE direction and the Caledonian Fracture Zone striking WNW-ESE with several secondary fault structures in direction N-S to NE-SW (Lemke, 1998). In the central Arkona Basin, about 1-2 km of sediments cover Precambrian granite of the Baltic Shield found in the "Petrobaltic" core G14. Tertiary and pre-glacial Quaternary sediments have been influenced by the main fluvial channel referred as "Baltischer Hauptstrom" transporting quartz sands and gravels from the south and middle of Sweden and the northern parts of the Baltic. The Quaternary evolution of the area is dominated by the glacial and interglacial stages. The Elster, Saale and Weichsel glacial stages formed the relief structure of basins and crests and the central trough of the Baltic, which served as the main channel for the progressing and retreating Scandinavian glaciers. 


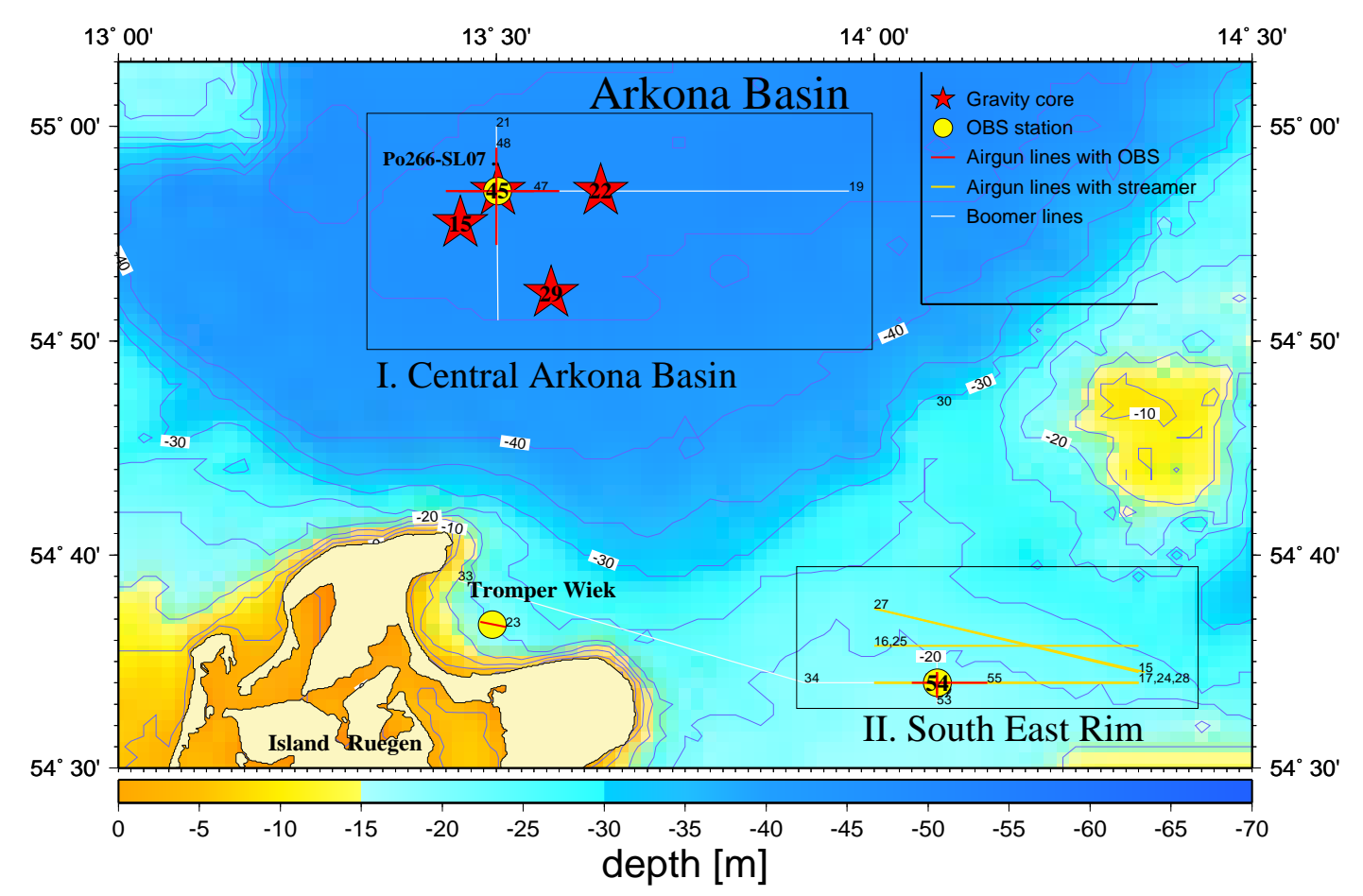

Figure 8.1: Location map of selected seismic lines and gravity coring and OBS positions in the two study areas in the Arkona Basin. Also marked is the location of the OBS station in the Tromper Wiek and the 'Petrobaltic' well G14. 
Following the retreat of the last glacial maximum of the Weichsel stage ( $\approx 20.000 \mathrm{BP})$, the melting of the ice produced a network of lakes eventually forming the Baltic Ice Lake. After the final drainage of the Baltic Ice Lake during the Younger Dryas several phases of marine transgression and blockages occurred with varying water levels. These include the Yoldia, Ancylus and Litorina stages which can be identified in the sediment stratigraphy of the high resolution seismic measurements. A detailed description of the sediments and the classification is found in Moros et. al. (Moros et al., 2002).

Most previous research activities in the Arkona Basin from the IFG have focused on the area of the central part of the Arkona Basin, marked as area I in fig. 8.1. Gravity coring and geophysical probing with the GISP have been conducted in the MAST project (Theilen et al., 1993). This provided a good base for further high resolution seismic profiling and gravity coring to obtain detailed information of the study area. The shallow water depth of 40-50 $\mathrm{m}$ is well suited for obtaining high resolution seismic data as well as for the operation of the long streamer, deployments of OBS stations and sediment sampling. The length of the streamer was believed to be sufficient for the deep-tow experiments in this area. However, due to material failure the streamer length had to be reduced to $2 / 3 \mathrm{rd}$ of the original length. Therefore the deep-tow experiments have been performed in shallower waters in the second area of investigation towards the south-eastern rim of the Basin. A very dominant hard reflector corresponding to base of quaternary (Upper Cretaceous) was expected to be close to the surface in this area. This would provide good conditions to generate Scholte or Stoneley interface waves as well as PS-converted waves. Therefore the south-eastern rim of the Arkona Basin marked as study area II in figure 8.1 was chosen as a suitable area to investigate the possibilities to excite and record Scholte waves with this streamer configuration.

The data presented in this case study was acquired during numerous cruises on the research vessels FS Alkor and FS Poseidon. The data sets presented are denoted by the first letters of the research vessel and the official cruise identifier, namely Po266, Al188 and Al201, and a number of the station or seismic line at the end, separated by a P for seismic lines, SL for gravity cores (Schwerelot) and OBS for ocean bottom stations. A list of all cruises is found in the appendix B.1.1. The location of the seismic lines and stations discussed in this chapter are marked on the location map in figure 8.1.

\subsection{A Towed Data Acquisition System}

The investigations of the previous case studies indicate that it could be feasible to measure dispersive interface waves within the water column. It was shown that the hydrophone component of ocean bottom receivers recorded sufficient Scholte wave energy which was excited by surface towed airguns (see chapter 6). Also we have evidence of a streamer record with Scholte wave energy in shallow marine environment from the Laptev Sea data set described 
in the previous chapter (Hinz et al., 1997).

The theoretical and numerical investigations on the energy distribution of Scholte wave energy have shown that the amplitude of interface waves decays exponentially with distance to the interface. Furthermore we expect the high frequencies to decay more rapidly than lower frequencies. Hence, in order to obtain measurements spanning the required frequency range to depict the velocity variation with frequency, we require to measure as close to the sea bed as possible.

System requirements and specifications This is limited by practical consideration and the acquisition systems, which lead to the following system requirements. The towing procedure close to the sea bed requires depth control and monitoring of the receiver system. Due to the proximity to the sea bed, circumstances might arise, where bottom contact of the towed system cannot be avoided. Therefore, the system must be robust to withstand such contacts without damage, and without environmental risk. This implies, that the use of conventional oil-filled streamers is not suitable for such experiments. In case of ground contact it is also of importance, that the towed system cannot get caught at obstacles on the sea floor. Systems with variable diameter between cable and acoustic nodes are more likely to be caught than systems with uniform diameter and smooth connections.

A new solid-state-streamer system was purchased and tested for the deep-tow experiments. The new solid-state-streamer consists of modular configurable sections with a maximum total towing length of $600 \mathrm{~m}$, which is comprised of 3x100 m lead-in and 3x100 m active sections. The tail buoy was attached with a 50-100 m long line. Since a minimum of two turns of lead-in cable have to remain on the winch only $250 \mathrm{~m}$ can be deployed at sea. Hence, we obtain a maximum length of the system of $\approx 600 \mathrm{~m}$, with active channels in the range of $250-550 \mathrm{~m}$. The following requirements and specifications can be summarized.

- The group interval of $3.125 \mathrm{~m}$ is sufficient small to provide adequate spatial sampling to apply the wave field transform.

- The length of the active section of $300 \mathrm{~m}$ is just sufficient to resolve dispersive modes in the range of interest.

- The length of the tow-leader (up to $250 \mathrm{~m}$ ) is sufficient for towing at depth in the NATLAB study area.

- An active depth control system can be used to monitor and control the depth position of the streamer.

- The overall size and weight of the system can be handled by medium size research vessels available. 
- The solid-compounds are no potential hazard to the marine environment in case of rigid bottom contact.

- The streamer has uniform diameter from the beginning to the end of the active section.

\subsection{Acquisition Tests}

First acquisition tests with a newly acquired solid-state-streamer (delivered in Feb. 2001) have been performed in the NATLAB study area in numerous research cruises in 2001 and 2002. The first deep-tow experiments could be conducted on cruise Alkor 188 in August 2001 and the following acquisition parameters were tested:

- the optimum towing depth of the streamer, as a function lead-in cable length, ship speed and depth control system parameters,

- the offset range of active channels.

\subsubsection{Towing Depth}

The towing depth of the deep-towed streamer is determined by three main factors. The gravitational force acting on the tow-leader to submerge the streamer, the length of the tow-leader and the uplift of the towed system related to the speed of the vessel. This depends on the natural buoyancy of the streamer as well as its cross section. The fine tuning and controlling of the streamer depth is achieved by the application of active streamer birds chartered from the University of Aarhus. These active depth controller units, denoted as "streamer birds", can operate their fin angle in the range of $\pm 15^{\circ}$, which can be operated remotely from the laboratory of the research vessel. Furthermore they are programmable to automatically adjust the fin angle in order to maintain a certain water depth. However, the uplift generated by the fins is determined predominantly by the towing speed. Therefore numerous acquisition tests had to be conducted, in order to determine the appropriate parameters to control the towing depth.

The amount of lead-in cable deployed for the measurements therefore controls the towing depth and the distance to the first channel of the active section of the streamer at the same time. Hence, the offset range towing depth and ship speed have to be chosen in compromise to obtain the acquisition geometry as close as possible to the requirements of the wave-field transform.

The active sections were built to be neutrally buoyant in fresh water, while the leadin sections are heavier and act as a depressor. Therefore it was assumed, that the active sections towards the end of the streamer would remain in the depth of the beginning of the 
active section since the gravitational forces are compensated by the buoyancy of the streamer sections. The level should be determined by the length of the lead-in section and the towing speed (Fig. 8.2a). The position of the streamer birds along the streamer is indicated in the lower images b) and c). Fig. 8.3 shows the depth recorded at each of the four depth controllers (top) and their fin angles (bottom) as a function of time. During the first test $200 \mathrm{~m}$ lead-in cable was deployed, but the full length of $260 \mathrm{~m}$ was required to stabilize the streamer at 20 $\mathrm{m}$ depth. The first depth controller (red line) could not obtain the nominated towing depth of $25 \mathrm{~m}$ despite of the maximum downward fin angle. Furthermore the towing depth was not very stable as seen in the periodic fluctuations as well in the depth of the controllers as for the fin angles. With full lead-in length at $3 \mathrm{kn}$ a stable condition could be achieved for a towing depth of $20 \mathrm{~m}$ just after midday. The fin angles indicate that the middle of the streamer tends to greater depth (green and blue lines) while the beginning and the end remained stable (red and magenta lines). Hence, the tendency of the streamer to float in an U-shaped manner as indicated in $8.2 \mathrm{~b}$ was observed.

Due to malfunctioning of parts of the streamer, only two active sections and two lead-in sections could be used for the rest of the cruise. The shortened configuration as indicated in 8.2c was used. With this configuration a maximum towing depth of $15-18 \mathrm{~m}$ could be achieved. Therefore, the first data acquisition was performed at water depth of $25 \mathrm{~m}$ at the

a)

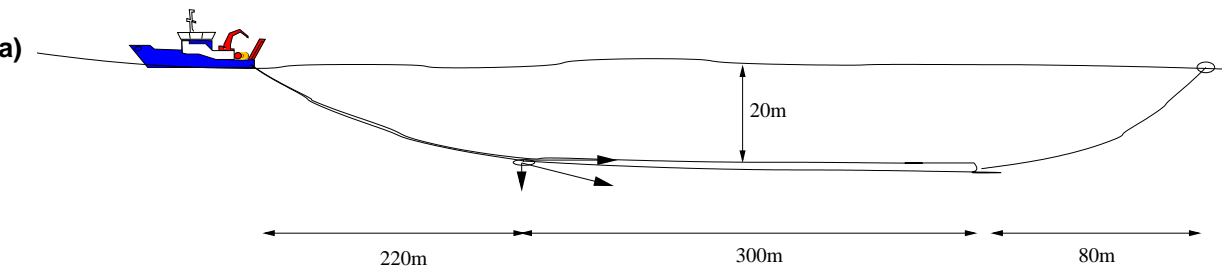

b)

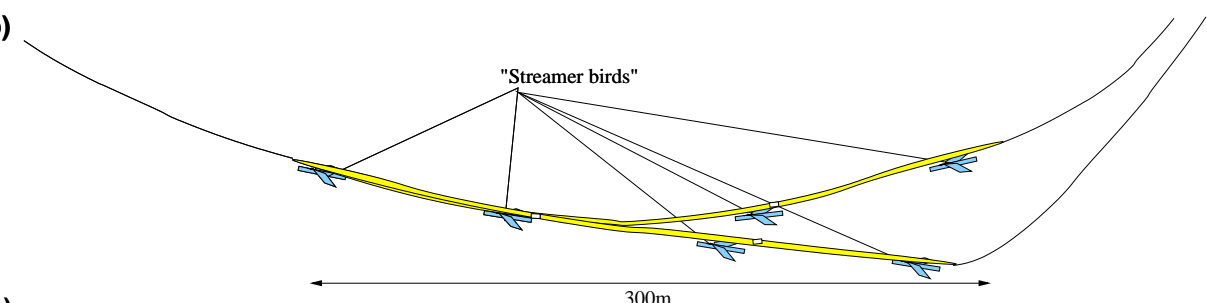

c)

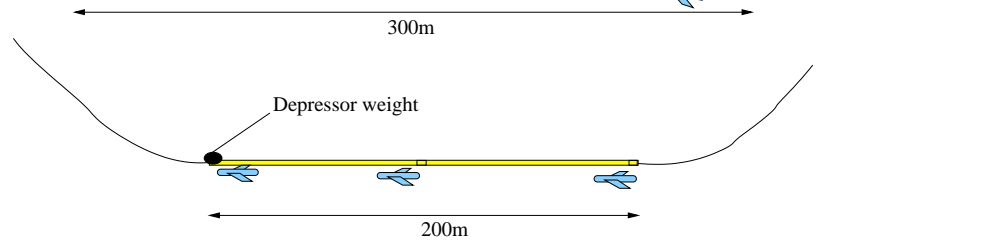

Figure 8.2: The towing-depth of the streamer is determined from the forces acting on the system as indicated by the arrows (a). The observed shape of the towed streamer (b) could possibly be improved by the application of a depressor weight (c). 


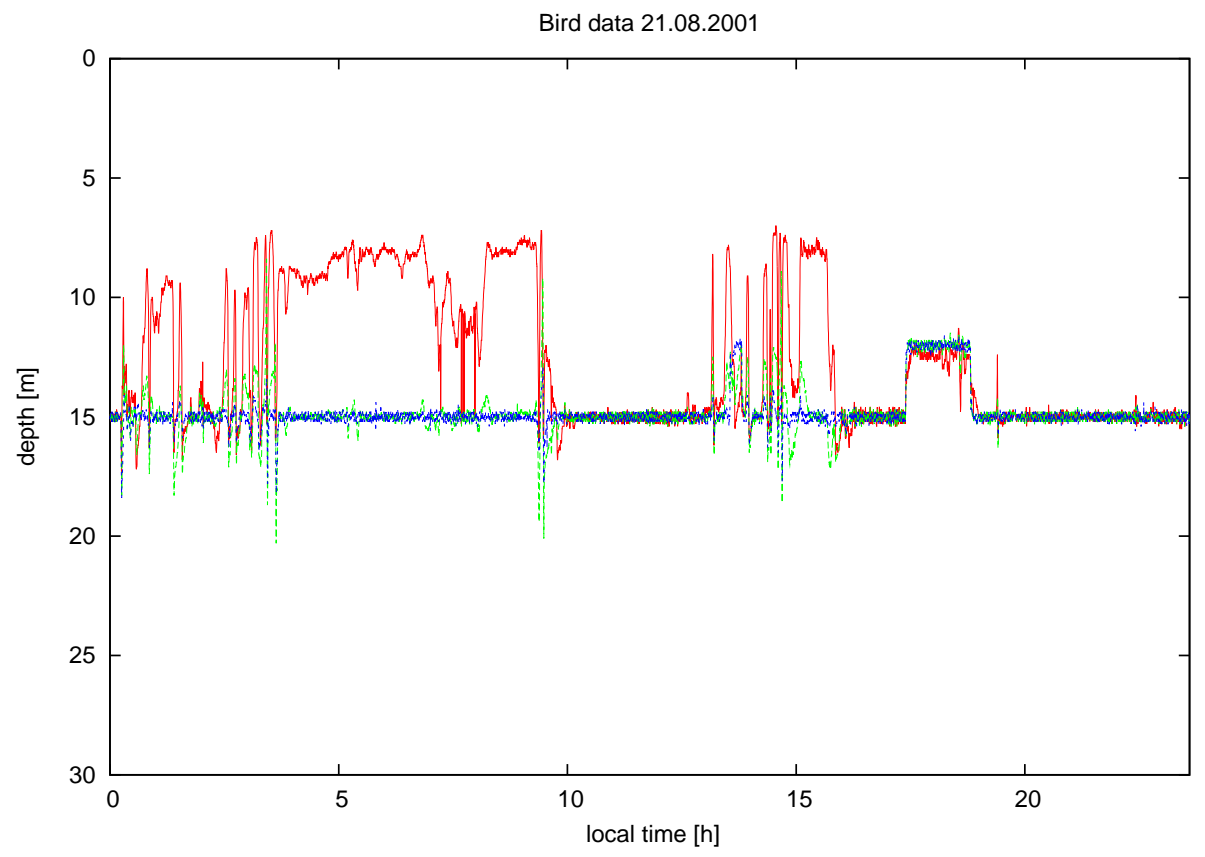

Figure 8.3: Depth control of the deep-towed streamer was monitored with a) the depth of the four streamer bird and $b$ ) the fin angle for each of the birds. 
south-eastern rim of the Arkona Basin.

\subsubsection{Offset Range}

Another limitation encountered with a streamer configuration in contrast to OBS recordings is the limited offset range of the gather. The streamer has a maximum length of active sections of $300 \mathrm{~m}$, corresponding to the discussion on limited spreads in section 3.3.1. Since the length of the lead-in cable was used to determine the towing depth of the streamer, this parameter could not be changed significantly during the acquisition tests. The initial offset range was intended to be from 170 to $470 \mathrm{~m}$. Due to the malperforming of the streamer sections only the offset range from 120-350 m could be investigated in deep-tow experiments at the southeastern rim of the Arkona Basin. Further experiments on different offset ranges would require to change the depressor weight of the lead in sections.

\subsubsection{Data Sets}

During the first data acquisition tests conducted on cruise Alkor 188 in August 2001, only 64 of the 96 channels were available within $200 \mathrm{~m}$ distance. Additionally, several channels and preamplifiers stopped working, until in the end only $60 \%$ of the channels were operational. Hence, the operation of the new streamer was not without complications, but nevertheless some useful data sets could be acquired. For the deep tow experiment the maximum possible lead-in length of $170 \mathrm{~m}$ was deployed. The towing depth of the streamer was varied between 15 and $22 \mathrm{~m}$ and the airgun source was deployed at $8-20 \mathrm{~m}$ about $50 \mathrm{~m}$ behind the vessel. The offset range covered by this configuration was about $120-320 \mathrm{~m}$.

Due to the shallow water environment, the arrival times for the direct wave and the seasurface ghost as well as the sea-floor reflection differ only by a few milliseconds. Additionally, at offset exceeding $100 \mathrm{~m}$, the refracted wave becomes the first arrival prior to the direct arrival. Hence, while generally the direct arrival could be used to check the acquisition geometry and the floating depth of the streamer, the identification of the direct arrival may not always be clear. The arrival times for the direct wave, surface ghost and sea floor reflection have been calculated and indicated as solid lines in the shot-gather shown in figure 8.4. In the time reduced section (the assumed velocity of water of $1450 \mathrm{~m} / \mathrm{s}$ was used) to the right (Fig. 8.4b) it can be seen, that the computed arrivals only differ by a few milliseconds, while the wavelets are much longer. But it is obvious, that the direct arrival is not in a straight line, but retarded signals occur at $250 \mathrm{~m}$ offset, hence just a few meters ahead of the position of the second streamer bird. The cause for this delay is not clear, but it indicates that the steamer was possibly not floating in a straight line. A suggestion to reduce the bending of the streamer within the active section is to add a depressor weight at about $3 / 4$ of the length of the lead-in section, in order to obtain the bend in the lead-in section rather than the active section. 


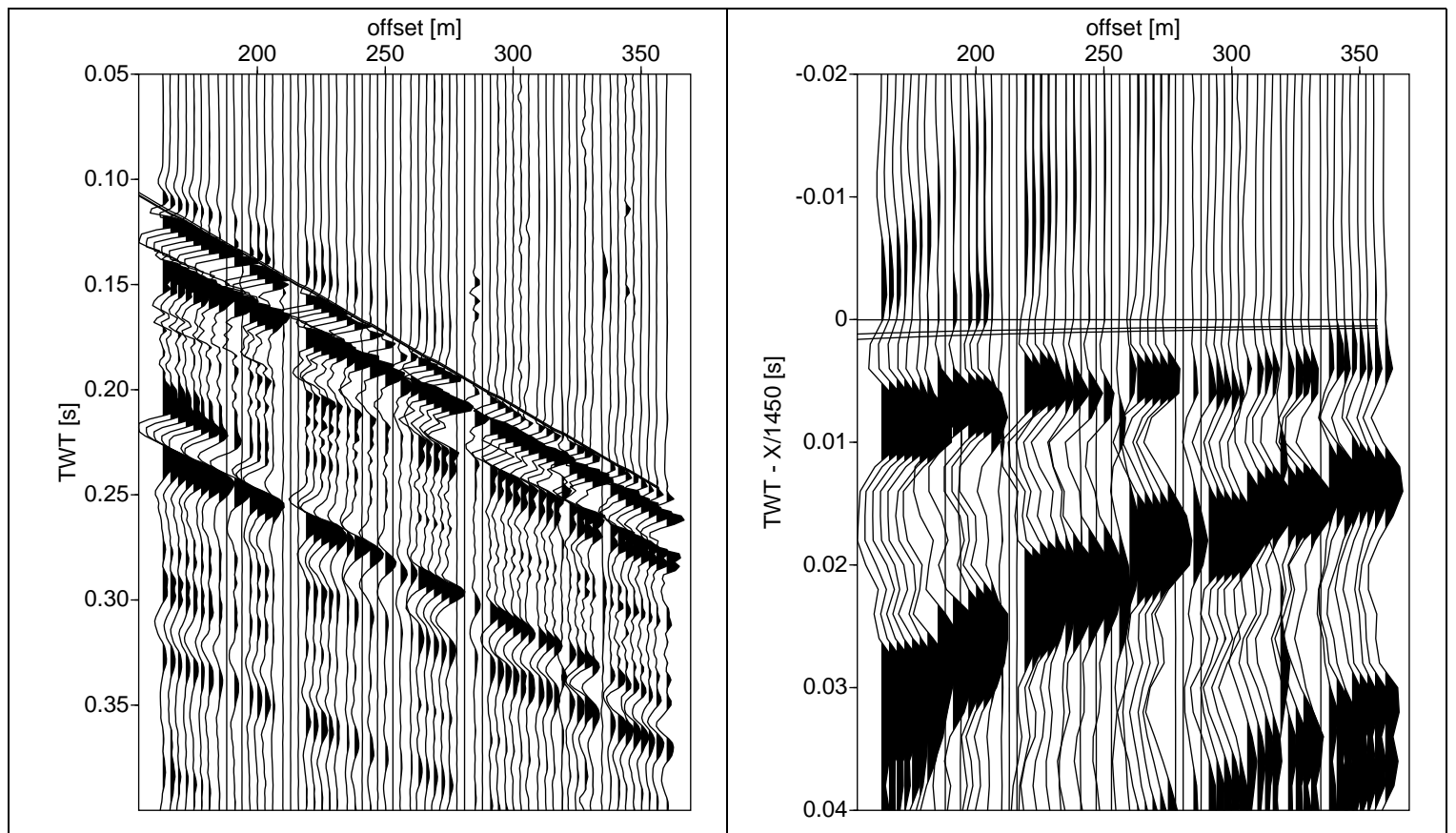

Figure 8.4: Arrival times of the direct wave and ghost arrivals for the deep-tow streamer configuration. 
Additionally removing the tail buoy and replacing it by a swim-line which will just float by itself could straighten the active sections, since it would not cause any upward pull on the tail of the active sections. Alternatively a small depressor weight about 1/4 of the length of the tail line could straighten the active section, but very likely at the cost of additional noise.
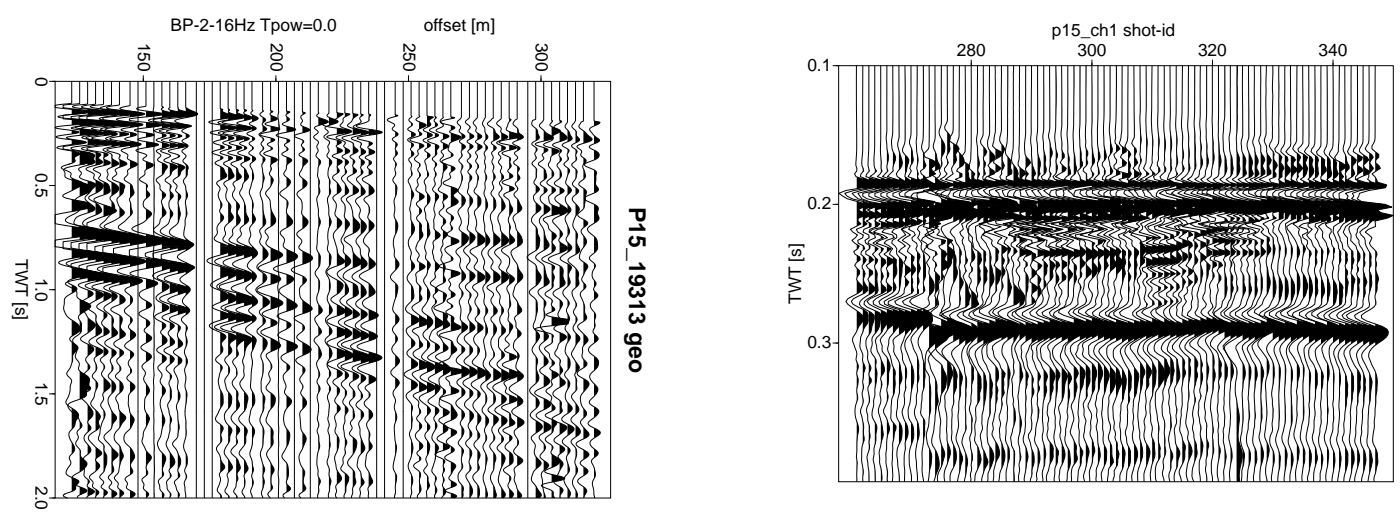

Figure 8.5: a) Deep-tow streamer gather and b) single-channel section of channel 33 (220 m offset)

The common-shot and single-channel sections in figure 8.5 are data examples from profile 15 of the south-eastern rim of the Arkona Basin. The shot-gather was low-pass filtered in order to enhance the dispersive interface wave, and numerous traces had to be eliminated or manually edited due to malfunctioning of hydrophones or preamplifiers in the acquisition system. The high amplitude and low frequency wave train beginning at $0.6 \mathrm{~s}$ at near offsets and ranging to 1.6 seconds at far offsets is believed to relate to an interface wave.

The wave train in the frequency range from 6 to $12 \mathrm{~Hz}$ propagated with group velocity of $200-500 \mathrm{~m} / \mathrm{s}$, but the phases correlate to phase velocities of $400-800 \mathrm{~m} / \mathrm{s}$. These are very high velocities and we believe that the dispersive seismic wave energy must be dominated by the influence of sub surface boundaries of the glacial till or chalk layers. This dispersive wave train was only observed in the range from shot 288 to shot 317 (Fig. 8.5b).

Further profiles in the south-eastern rim study area were acquired with different airgun sizes as indicated in fig. 8.6. On the seismic lines acquired with the intermediate size airguns of 0.3 and 0.61 chamber volumes no dispersive waves wave been identified. Some dispersive energy was observed along the profile P24 acquired with a 1.21 airgun and along profiles P15P17 shot with the largest airgun available $(2.51)$. The shot interval is reduced to $90 \mathrm{sec}$ for this airgun due to the high amount of compressed air required for shooting. The shot-to-shot distance is $180 \mathrm{~m}$, approximately one streamer length. 


\subsection{Determining Sediment Stratigraphy and Velocity Mod- els}

The sediment stratigraphy of the two areas was compiled from the information of the previous studies by Moros, Lemke and Endler, combined with the analysis of high-resolution seismic data and refraction analysis of the multi-channel seismic lines. Additional detailed information is used from the results of the gravity coring, which have been provided by the colleagues Olaf Thießen and Mark Schmidt from the NATLAB-INGGAS project. Here, the main focus is on the gravity core SL07 from cruise Poseidon 266 (Po266-SL07) since we have data from a successful OBS deployment from this location. Gravity coring was not possible in the south-eastern rim study area due to the hard surficial sediments. Sandy-silts were found in a grab sample taken near the site of the OBS deployment. The core locations in the central part of the Arkona Basin are marked as red stars in figure 8.1. I will continue with an introduction of the high-resolution seismic lines acquired for the stratigraphic analysis. This background information and refraction analysis results are then used to compile a starting model of the sub-surface structure to be used for the inversion of the dispersive seismic data.

The classification of stratigraphic sequences of the Arkona Basin was discussed in detail by Moros et.al. (1999), Lemke (1998) and Endler (1989). While Moros presented a

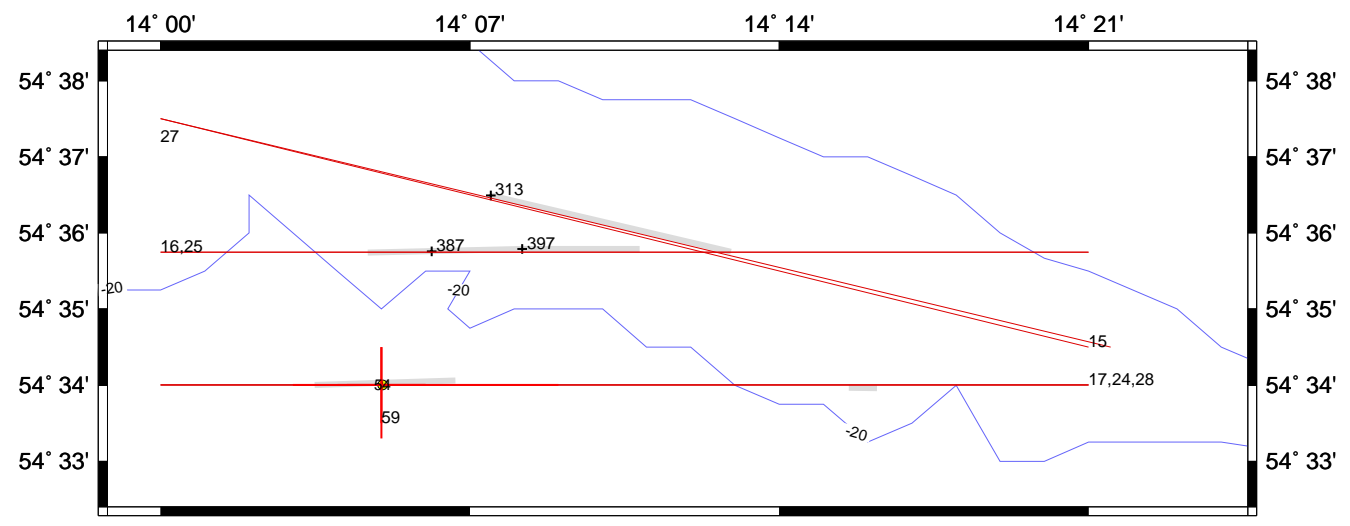

Figure 8.6: Seismic lines of the deep-tow experiments in the south-eastern rim study area. Airguns of 2.51 and 1.21 were used on profiles P15-17 and P24,25, respectively. The small airgun $(0.11)$ was used for surface towed streamer profiles P27-28. The location of OBS 54 is marked by the circle. The locations where interface wave energy was observed in the streamer sections is marked by grey patches along the seismic lines. The numbered crosses indicate the shot positions of the data examples from the deep-tow streamer experiments. 
lithostratigraphic classification based on the occurrence of dominant sandy layers, I followed the seismostratigraphic classification of Endler and Lemke as presented in table 8.1 (Moros, 1999, cf. for comparison of previous classifications). The sequences E1 and E2 are glacial

\begin{tabular}{c|c|c|c|} 
& Id & Baltic Stages & Sequence \\
\hline \hline & E5 & Littorina / recent & post glacial mud \\
& E4 & Ancylus / Littorina & grey clays with sandy-silt layers \\
E3 & Baltic Ice Lake / Yoldia & 'rosa' varvy clays with sand layers \\
E2 & Baltic Ice Lake & glacial till \\
E1 & Baltic Ice Lake & glacial till \\
\hline Ch & & chalk
\end{tabular}

Table 8.1: Stratigraphic sequences of the Baltic Sea classified according to Endler (1989) and Lemke (1998).

tills relating to glacial material carried by the discharge of the melting ice sheets and deposited in the Baltic Ice Lake. The sequence E3 includes the second phase of the Baltic Ice Lake and the Yoldia stages of the first marine transgression. The reddish-brown "rosa" colored warved clays of E3 are followed by post-glacial grey deposits of the Ancylus stage in sequence E4. This older interpretation is opposed by the Moros, relating the stratigraphic boundary to a distinct sand layer corresponding to the late Yoldia regression. The olive-grey soft muds of the Litorina stage and most recent deposits compose sequence E5 (Lemke, 1998). The base of the quaternary is marked by a dominant reflector relating to a chalk sequence. It is not always present in the boomer seismic data, but it is a dominant reflector in most sections, specially towards the shallower parts of the Arkona Basin.

\section{Seismostratigraphic interpretation}

These sequences have been identified in the high-resolution seismic lines of the various cruises. The sub-bottom profiler lines 19 and 21 acquired on cruise Al201 are data examples of the central part of the Arkona Basin shown in figure 8.7. Line 19 was shot from east to west direction while line 21 was crossing this line from north to south at the location of the gravity core sample Po266-SL07 taken during the Poseidon 266 cruise. The line drawings in fig. 8.8 are based on the above classification summarized in table 8.1 and interpret the seismic lines mentioned above. The line drawings were used to estimate the layer thicknesses for 1D start models at the OBS locations.

The stratigraphic situation of the second study area at the south-eastern rim of the Arkona Basin is very similar, but the thickness of Holocene sequences is much less. The hard sed- 


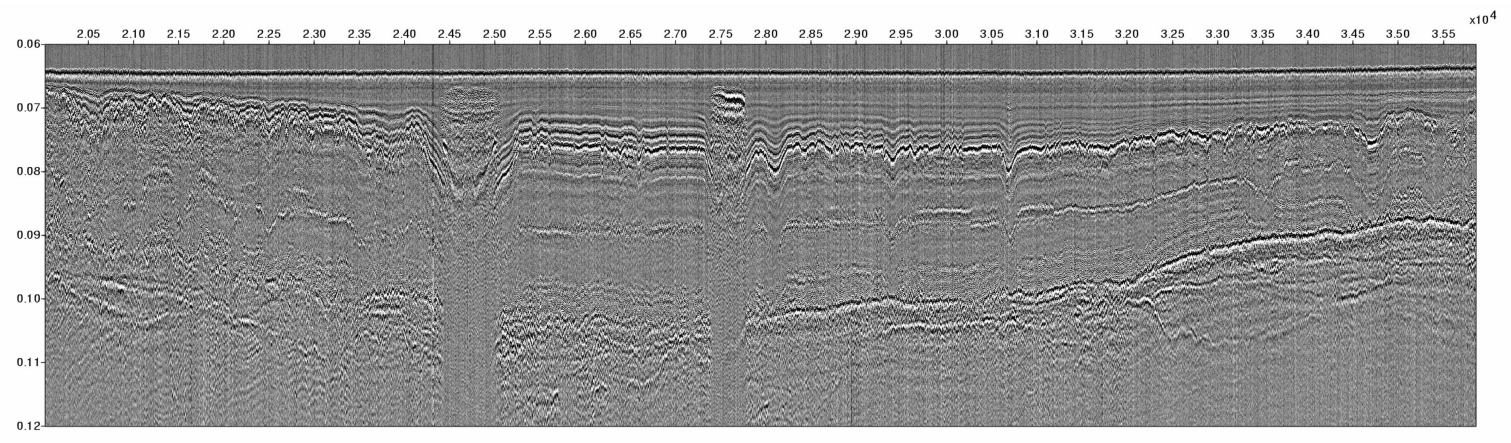

(a) Seimic line in N-S direction coming into the gas zone

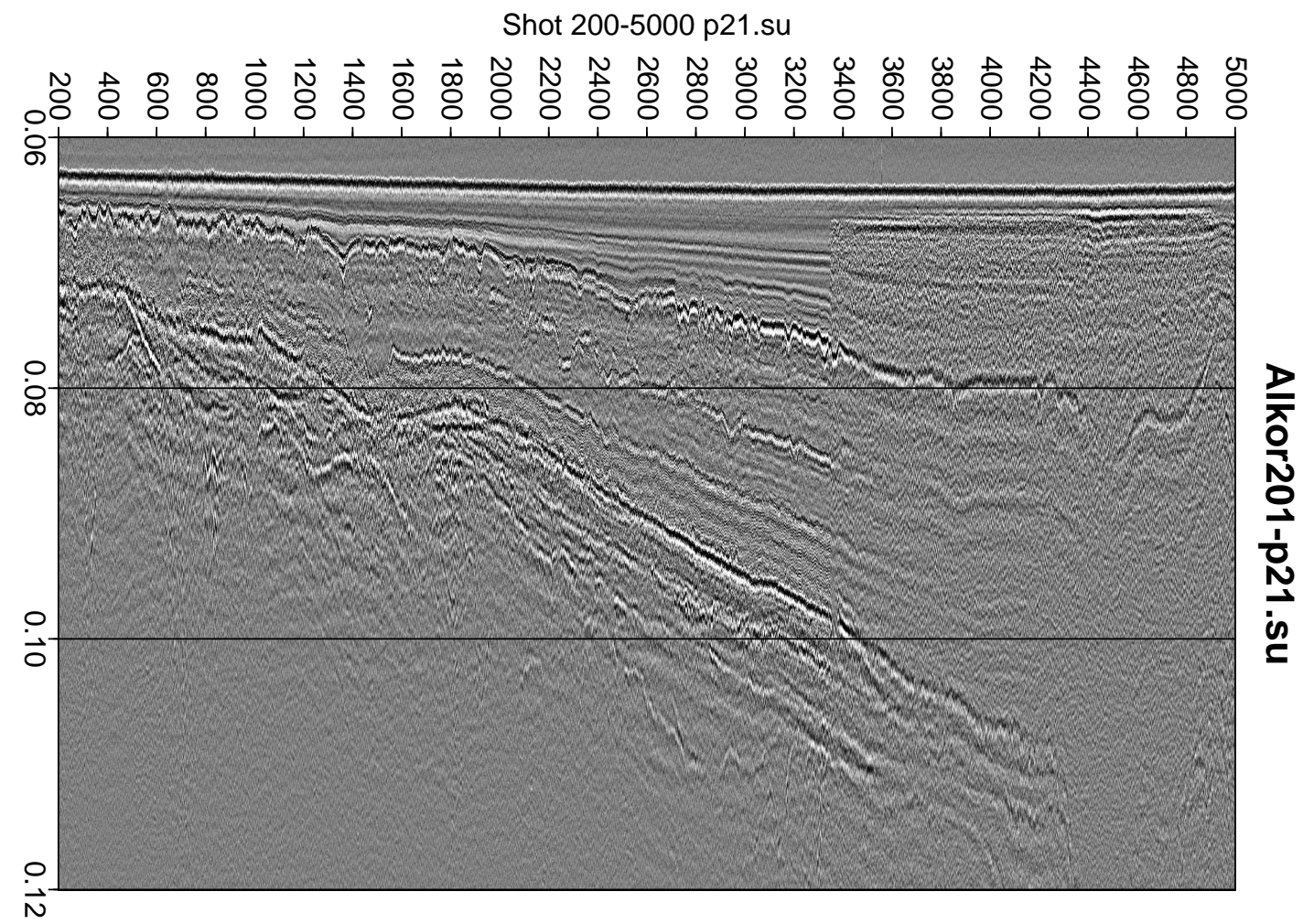

(b) Seismic line crossing the core locations and seismometer station from east to west.

Figure 8.7: High resolution seismic section coming into the central basin area. 


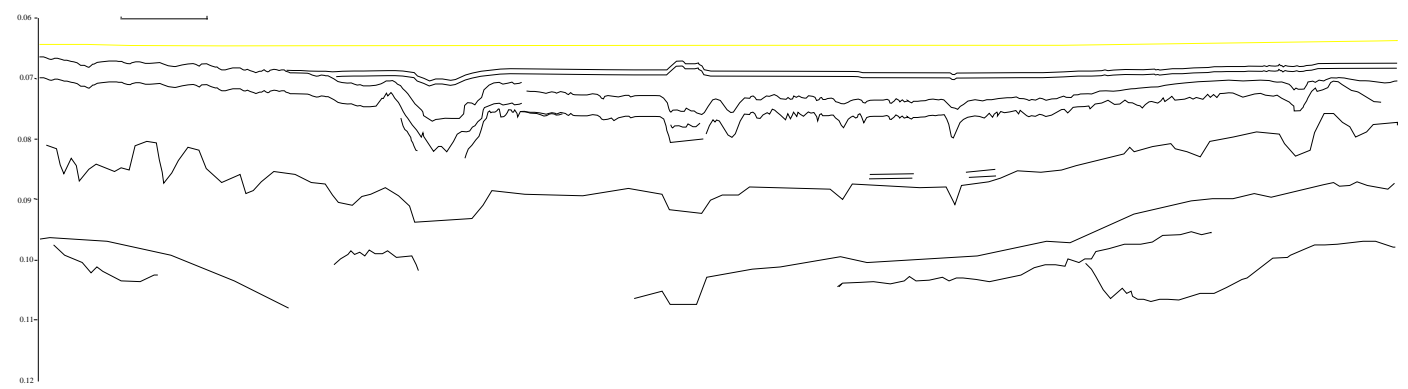

a) line drawing from Al201-P19

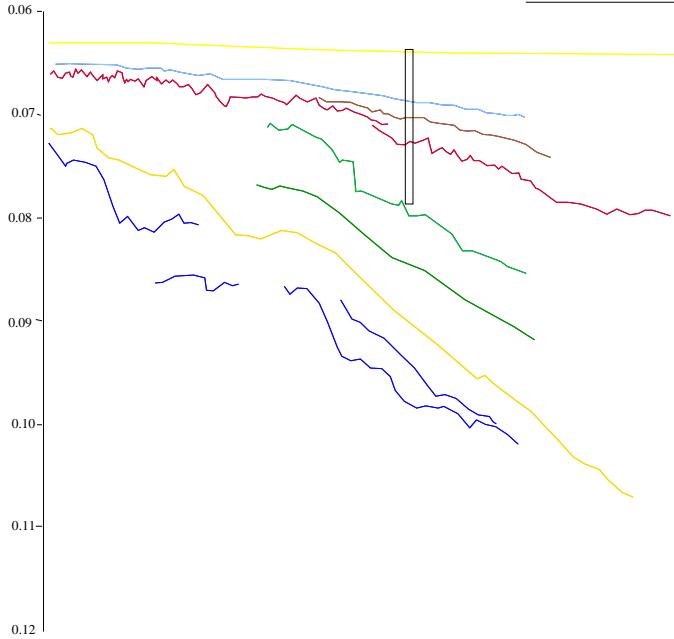

b) linedrawing from Al201-P21

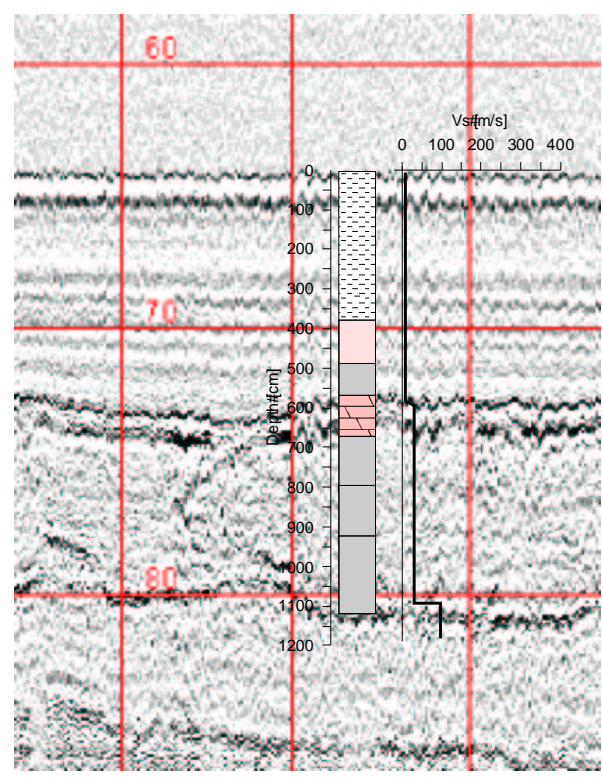

c) lithostratigraphy from gravity core Po266-SL07

Figure 8.8: Line drawings of high resolution seismic data sets in study area I. 
iment types of the glacial till and chalk layers are close to the surface as indicated in the seismic section and line drawing of profile P34 and P28.

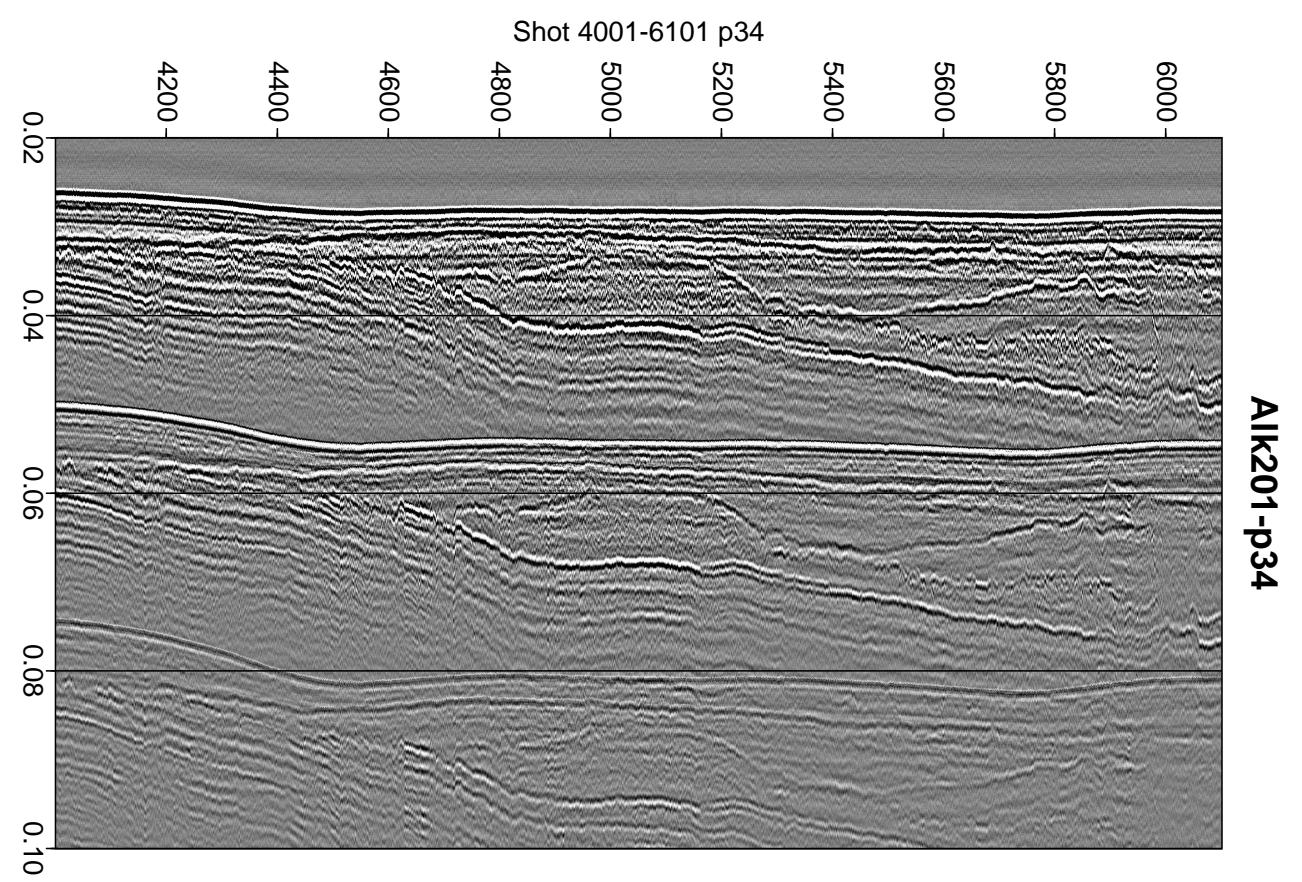

Figure 8.9: The sub bottom profiler section from study area II shows the structure of the dominant layers.

\section{Seismic refraction analysis}

Selected shot gathers from the multi-channel seismic (MCS) data sets have been used for seismic refraction analysis to determine the compressional wave velocities for the start model. Figure 8.11 shows an example from line P17. The calculated velocity of the surficial layer is ignored since the formula of the analysis tool expects source and receiver to be at the surface. Hence the low velocity in the surficial layer compensates the reduced travel times due to the receiver position at depth. Refracted first arrivals with apparent velocities of 1550, 17401900 and 2100-2400 m/s have been identified for the dominant layers on the MCS gathers of profiles A1188-P17 and -P28. These velocities have been interpreted as velocities of the sediments, the glacial till and the chalk layers in the stratigraphic model and are used for the starting models for the analysis of the dispersive seismic data. These one-dimensional models for the two OBS locations are shown in figure 8.12. 


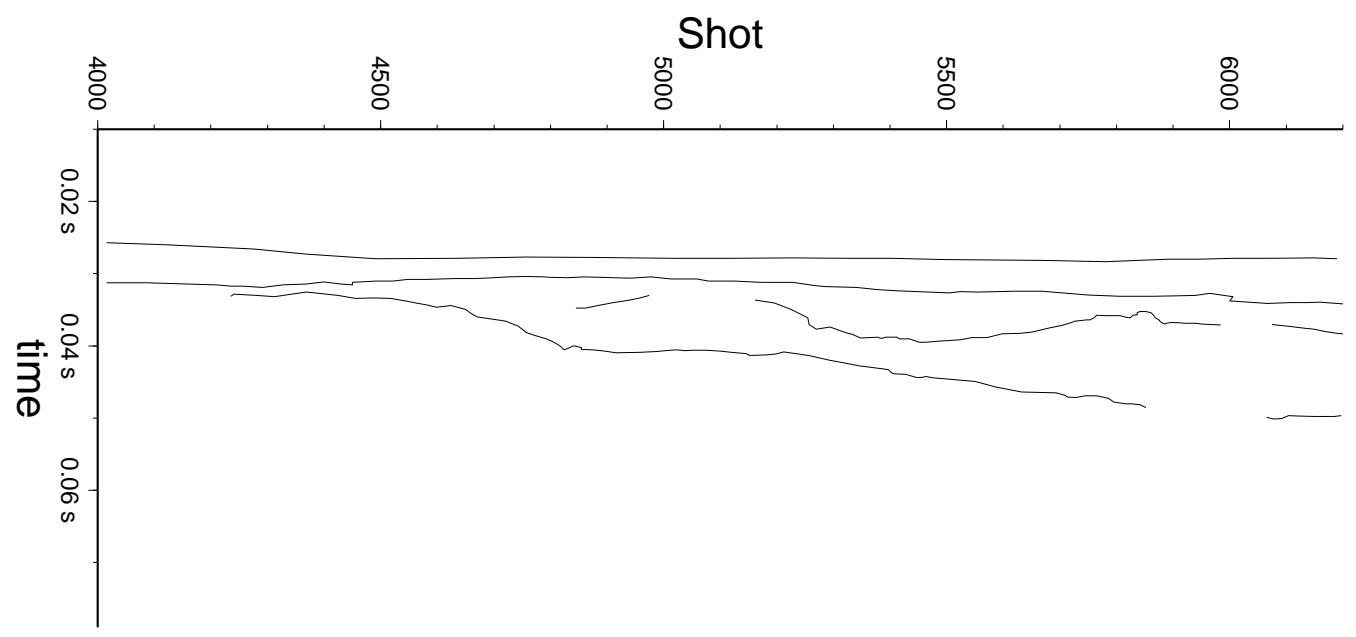

Figure 8.10: Line drawings of high resolution seismic data sets in study area II.

REFRACT V4.4 REFRACTIion seismics - data interpretation

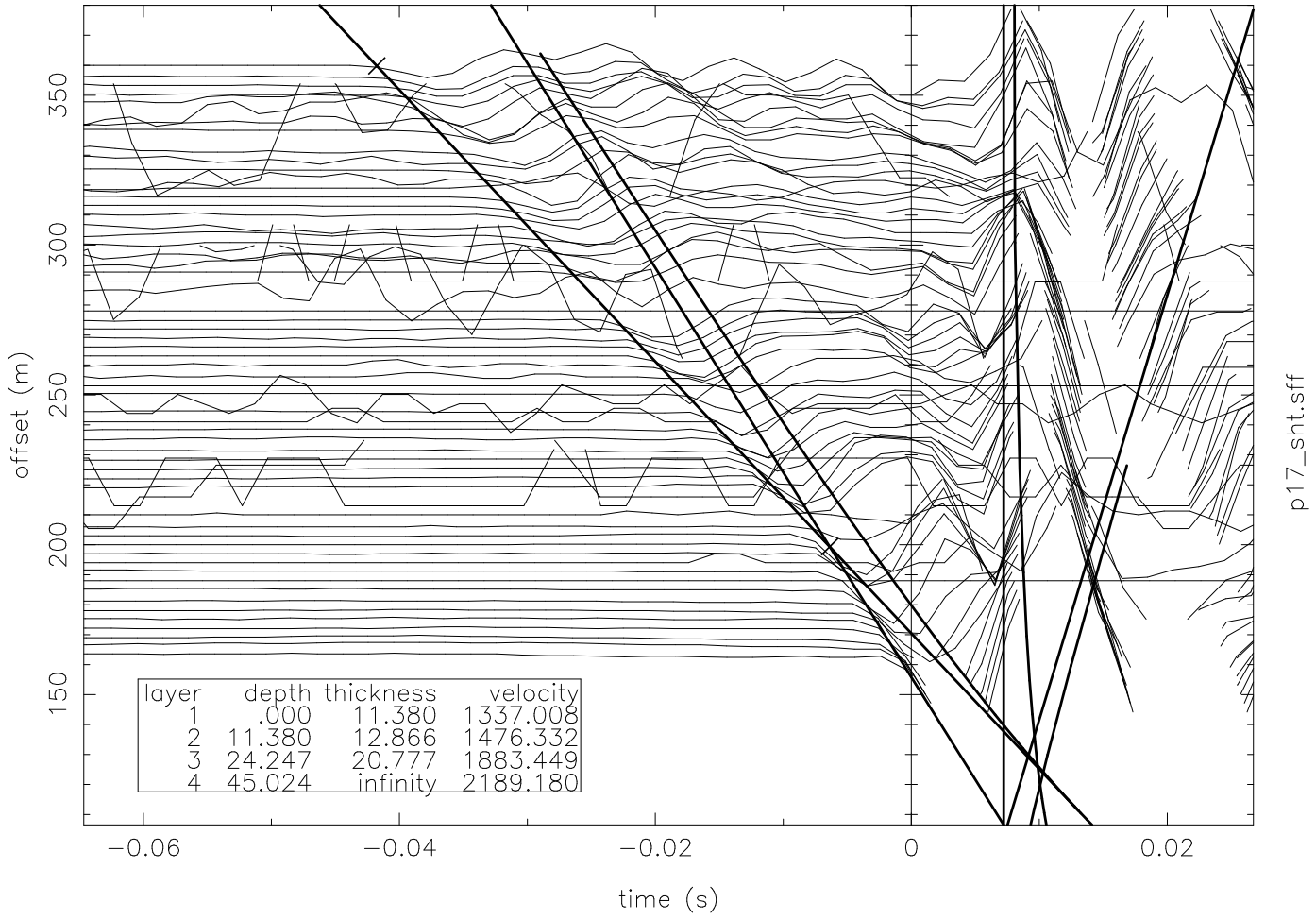

Figure 8.11: Refraction analysis of shot gather from data set II.2 


\subsection{Acquisition and Preprocessing of the OBS-Data}

During cruise Alkor 201 several OBS deployments were used to obtain dispersive seismic wave measurements comparable to the streamer measurements. Since the sediment physical data and the deep-tow streamer investigations had to be conducted at different sites, the OBS experiments were conducted in both study areas. The locations of the OBS stations in both study areas are marked by the yellow circles on the location map in figure 8.1. The OBS was deployed with a surface buoy. The seismic lines were shot with the 2.51 airgun in order to excite dispersive interface waves. Additional profiles were acquired using the small airgun and higher shot rates to obtain wide-angle reflection profiles for velocity analysis. From the results of the first case study we know that the sampling theorem is hard to fulfill in case of very soft sediments with low shear wave velocities. Since the presence of low shear wave velocities in the central Arkona Basin is known from previous studies (Theilen et al., 1993), it was doubtful, whether interface energy would be resolvable. However, the presence of Scholte wave energy should be detectable from the particle motion of the geophone components of the OBS.

The accurate position of the OBS stations and the source position had to be determined using the Differential GPS measurements and travel time inversion of the first arrivals of the seismic source. The influence of the accuracy of the navigation data was investigated

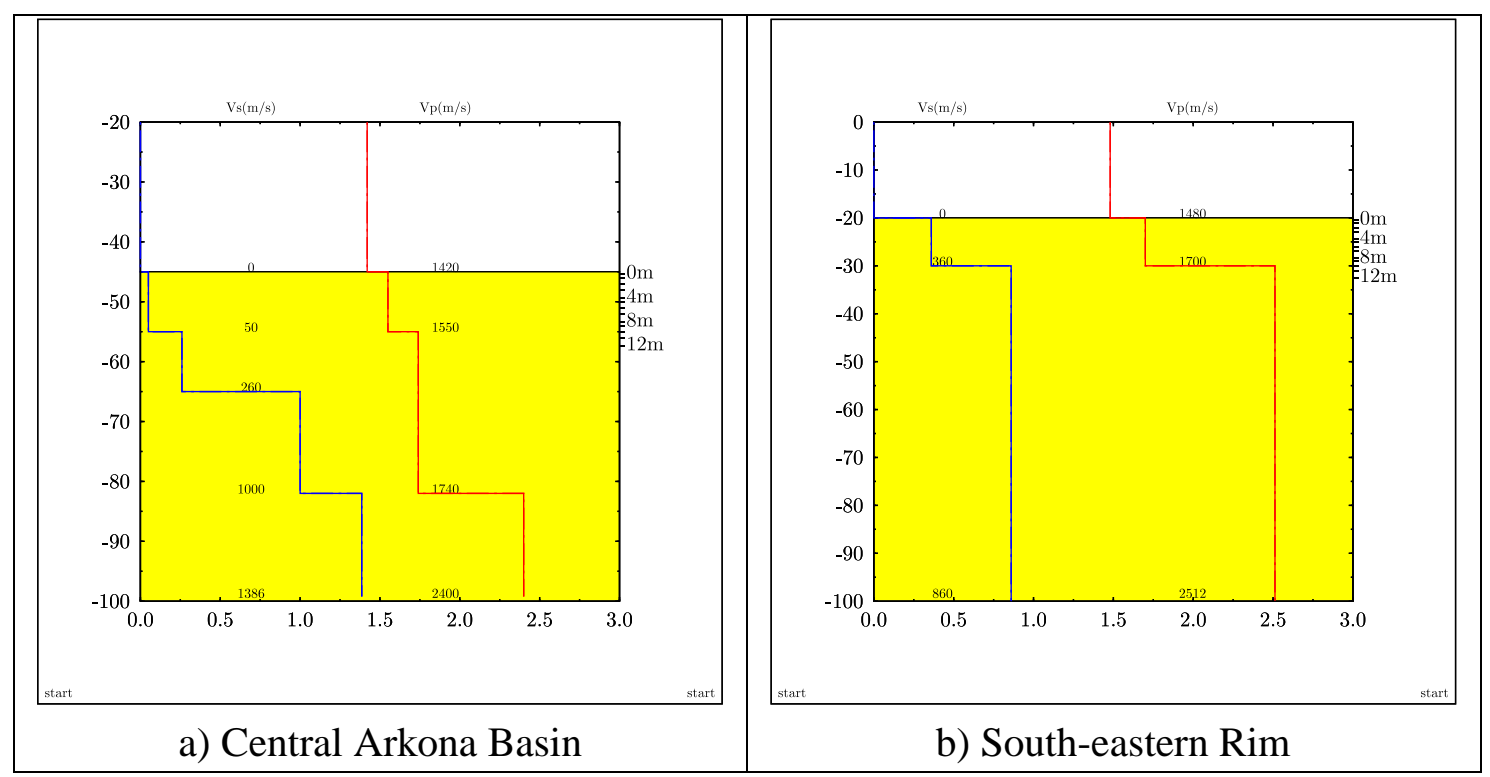

Figure 8.12: Start models for the two sites derived from the refraction analysis and interpretation of the seismostratigrahpic sequences. 
with a synthetic data set as well as the interpretation of the measured seismic data with a crude and more accurate navigation processing scheme. Both results indicated that the crude geometry with accuracy of +- $30 \mathrm{~m}$ for the OBS position is sufficient, only if the near offset traces up to $150 \mathrm{~m}$ offset are eliminated prior to the data processing. However, the data of this offset range may enhance the resolution of the data set significantly if used with an accurate geometry with errors less than $+-2 \mathrm{~m}$. This is documented in the series of wave field transforms of a synthetic data set with deliberately erroneous geometry settings presented in the appendix A.2.

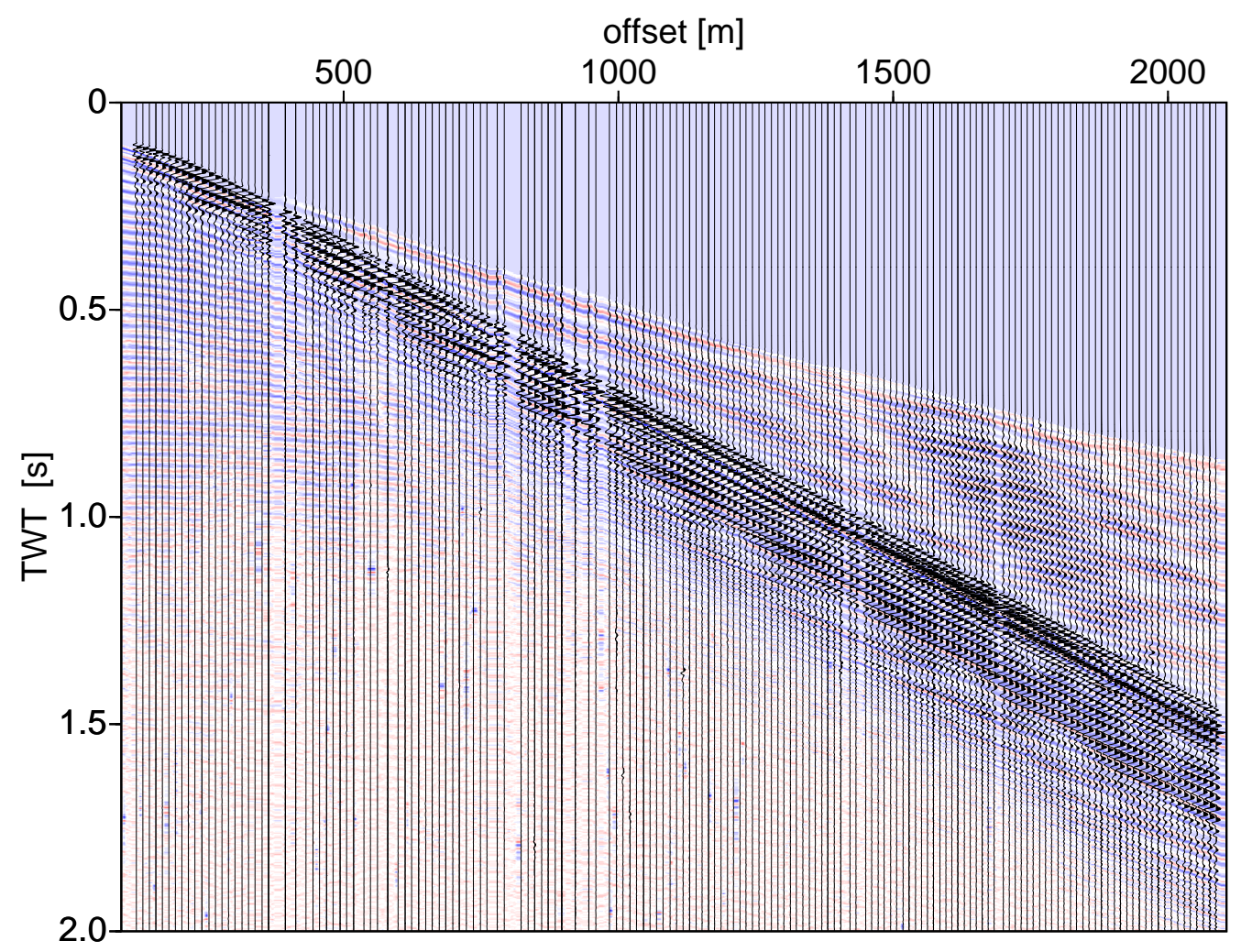

Figure 8.13: Common-Receiver-Gather of A1201-P47 recorded on OBS 45 in the Central Arkona Basin.

The common-receiver-gather of the OBS station 45 presented in figure 8.13 shows distinct dispersive modes, which are more clearly visible in the time reduced zoom of the section in 


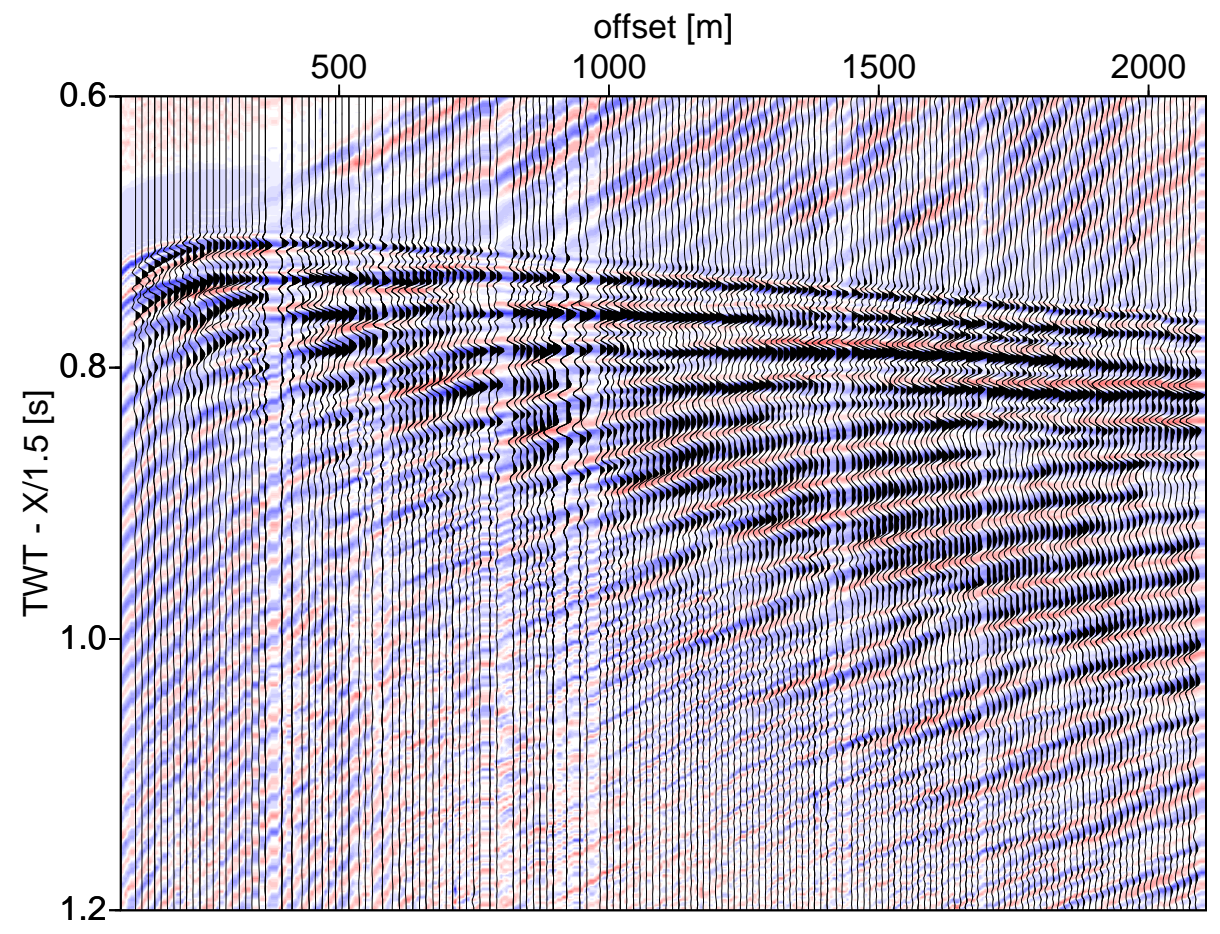

Figure 8.14: Time reduced zoom of the CRG in fig. 8.13.

The dispersive character of the acoustic wave train includes the nearly horizontal phases from 0.7 to 0.8 as well as the dispersive mode seen from 0.7 to $1 \mathrm{~s}$ along the offset range. 
figure 8.14. The mode propagating at group velocity of $1250 \mathrm{~m} / \mathrm{s}$ and phase velocity of $1600 \mathrm{~m} / \mathrm{s}$ is most dominant next to the mode with phase velocity of $1500 \mathrm{~m} / \mathrm{s}$ which aligns horizontally in the time reduced seismogram section. The velocity range suggests that these dispersive modes correspond to acoustic guided waves. Low velocity and low frequency wave energy corresponding to Scholte waves was not observed as visible in the low pass filtered section in figure 8.15. The OBS station was passed at $100 \mathrm{~m}$ distance, hence it can not be

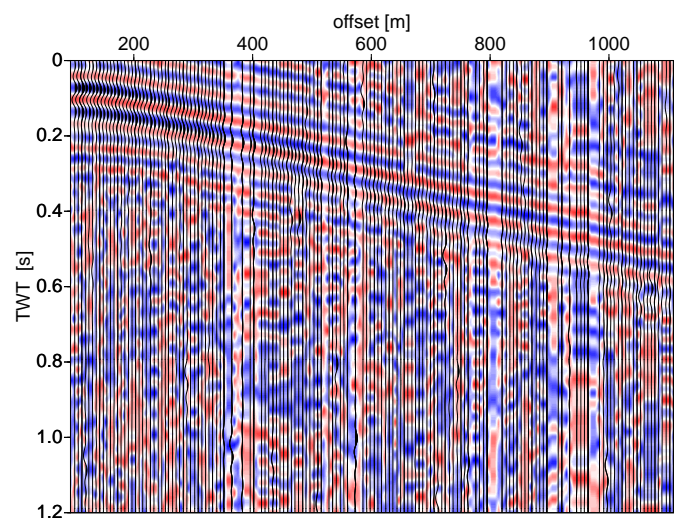

Figure 8.15: Low pass filtered section of Al201-P47 recorded at OBS 45. The wiggle traces are scaled depending on offset while the color image was AGC amplified. No Scholte wave energy is observed below $20 \mathrm{~Hz}$.

expected to identify Scholte wave energy traveling with a velocity of propagation of less than $50 \mathrm{~m} / \mathrm{s}$ at this spatial sampling $(10 \mathrm{~m})$ and distance.

In contrast to the section from OBS 45, the common-receiver-gather recorded at the OBS station 54 in the south-eastern rim area exhibits Scholte wave energy in the unprocessed raw data plotted with time and offset dependent gain in figure 8.16. The interface wave propagating with a group velocity of $160 \mathrm{~m} / \mathrm{s}$ and phase velocity of $600 \mathrm{~m} / \mathrm{s}$ is clearly visible and can be enhanced by low-pass frequency filtering (Fig. 8.17). The acoustic wave does not exhibit dispersive modes. Further analysis and identification of dispersive modes is investigated in the wave-field transformed sections in the frequency-slowness domain.

\subsection{Analysis of Dispersive Wavefields}

The first dispersive seismic waves measured with the new deep-tow streamer have been acquired on seismic lines P15 to P17 of cruise A1188 in August 2001. The on-board processing 


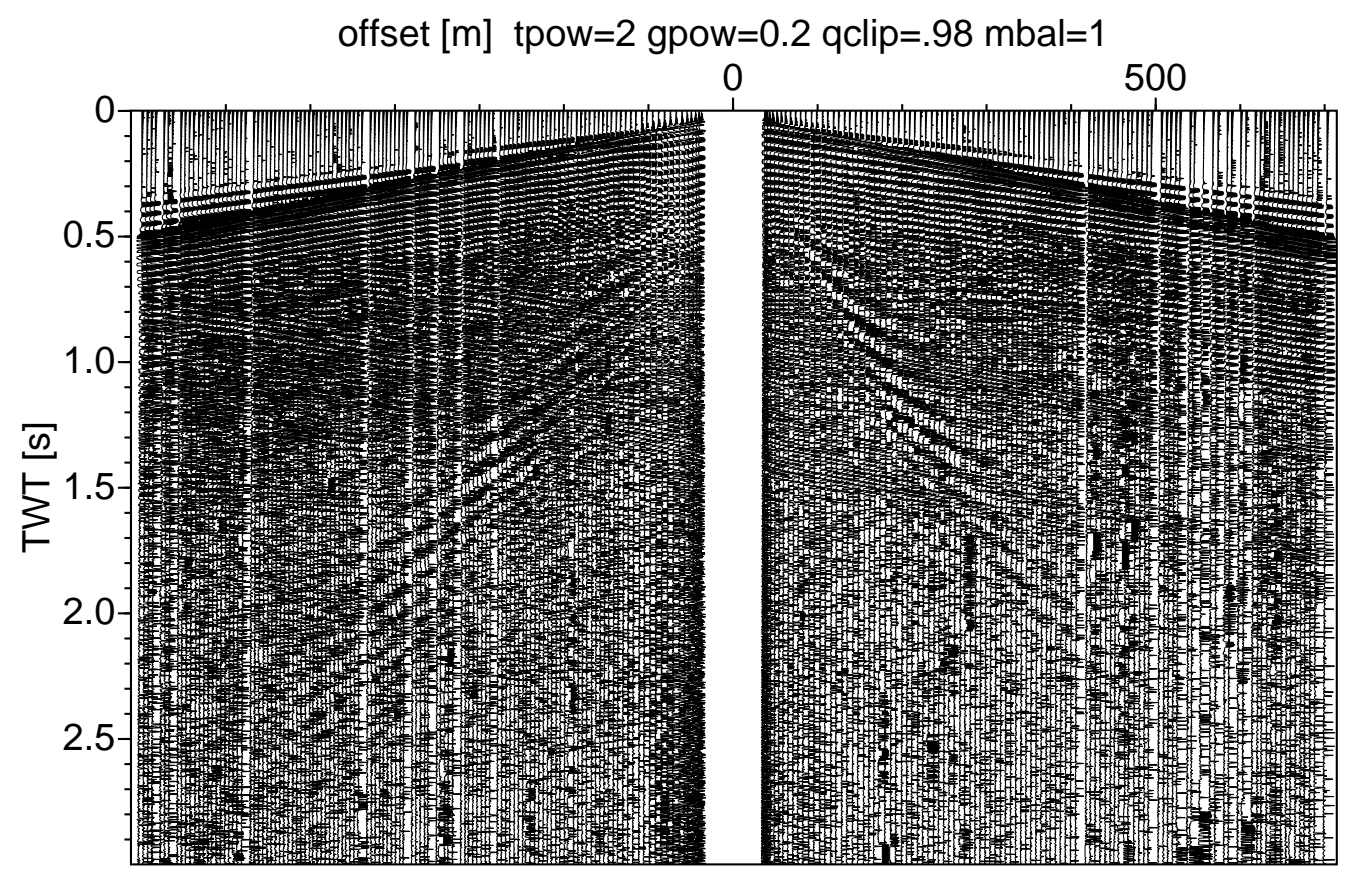

Figure 8.16: Common-receiver-gather of seismic line Al201-P59 recorded at OBS 54 in the South-Eastern rim study area. The dispersive interface wave visible in both branches of the split-spread recording has phase velocities of $400-800 \mathrm{~m} / \mathrm{s}$ and a group velocity of approximately $250 \mathrm{~m} / \mathrm{s}$. 


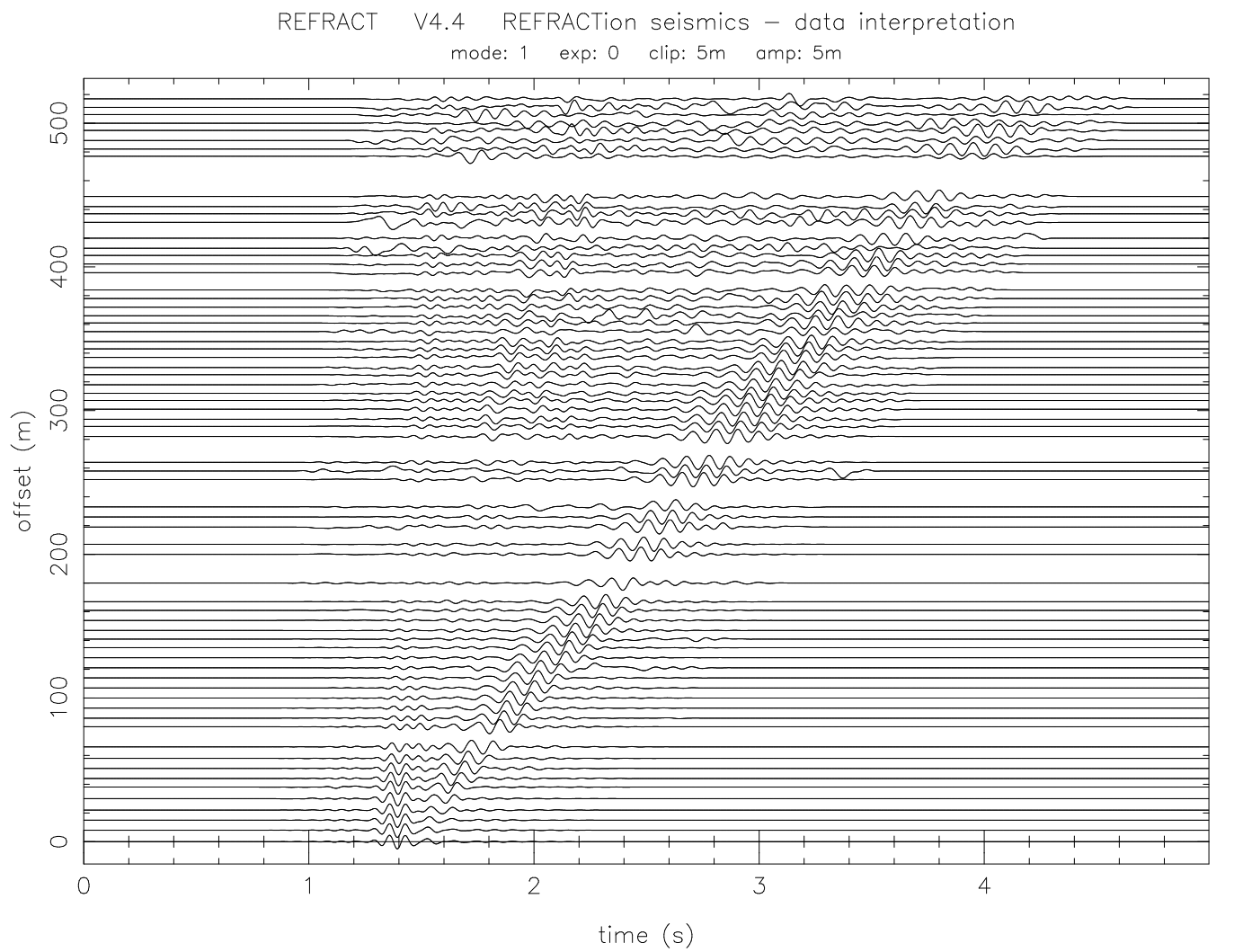

Figure 8.17: The interface wave of Al201-P59 (Fig. 8.16) has been enhanced by low-pass filtering and the application of time domain tapers. This is the input data set for the modified Fourier-Bessel transform. 
to identify the dispersive wave types was performed using standard processing tools of the Seismic-Unix package. Prior to the wave-field transform manual editing and amplitude normalization had to be applied, since high amplitude electrical noise was contaminating the shot gathers. Nevertheless the seismic wave field exhibits some dispersion in the frequency range from 6 to $12 \mathrm{~Hz}$ with phase velocity ranging from $400-800 \mathrm{~m} / \mathrm{s}$ ( Fig. 8.18, left).
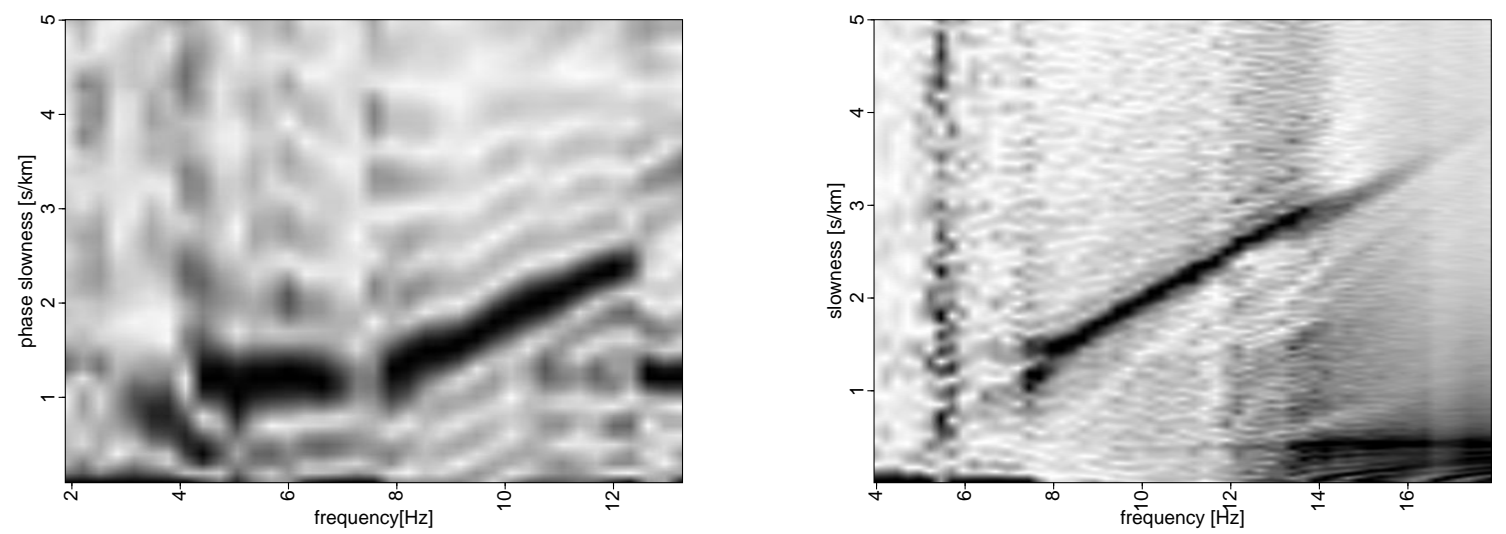

Figure 8.18: Slowness spectra of the deep-tow streamer shot 313 on seismic line A1188-P15 (left) and of line Al201-P59 recorded at OBS 54 in the south-eastern rim study area.

The spectral coefficients have been normalized for each frequency in both images.

These are very high velocities and we believe, that the dispersive seismic wave energy must be dominated by the influence of sub surface boundaries of the glacial till or chalk layers, hence could be classified as Stoneley waves according to Rauch (1980).

The comparison with the OBS data set of the same area confirms the dispersion characteristics found by the streamer measurements (Fig. 8.18, right). The slowness-spectra in figure 8.19 are obtained from the offset tapered common-receiver-gather (Fig. 8.17). The spectral coefficients have been normalized for each frequency. Therefore the dispersion relation can be identified at an enlarged frequency range. An acoustic wave is also present in the OBS data-set, but no low velocity Scholte wave energy can by identified in either of the two data-sets. The Scholte wave energy would be expected at slowness values exceeding $5 \mathrm{~s} / \mathrm{km}$ for low frequencies. Very distinct Scholte waves have been observed in the Tromper Wiek study area. However the image to the left indicates that no interface wave is present at this frequency-slowness range at this site.

The streamer recordings along profiles P15, P16 and P17 show slight variations in the position of the dispersive mode in terms of frequency and slowness values as well as their amplitude. Furthermore is the dispersive wave energy only present if the strong reflector 
seen in the single channel section of figure 8.5 had strong amplitudes in the seismic section. This coincides with the bathymetric high of the sea floor along this seismic line, which also enhances the amplitudes of the dispersive waves since the relative distance between the streamer and the sea floor is reduced. Two slowness-spectra of shot sections about $2 \mathrm{~km}$ apart are shown in figure 8.20.

The dispersive guided waves of the seismic wave-field observed on the OBS in the central Arkona Basin are clearly visible in the wave-field spectrum in figure 8.21. The amplitude variation of the individual modes indicates interference and can contain important information on the sub surface layers. While the fundamental mode as well as the first and third higher modes have energy at all frequencies the second higher modes disappears at frequencies above $65 \mathrm{~Hz}$. From the modal pattern one would expect a mode starting at cut-off frequency of $75-80 \mathrm{~Hz}$ and $0.4 \mathrm{~s} / \mathrm{km}$, but no dispersive mode is observed at this frequency.

The amplitude pattern below $0.4 \mathrm{~s} / \mathrm{km}$ at the continuation of the third higher mode (at about $45 \mathrm{~Hz}$ ) indicates the presence of another wave guide. I denoted this pattern as feathering in the figure caption, but in fact it resembles the pattern of the fundamental and higher modes of the water waveguide just at lower p-values.
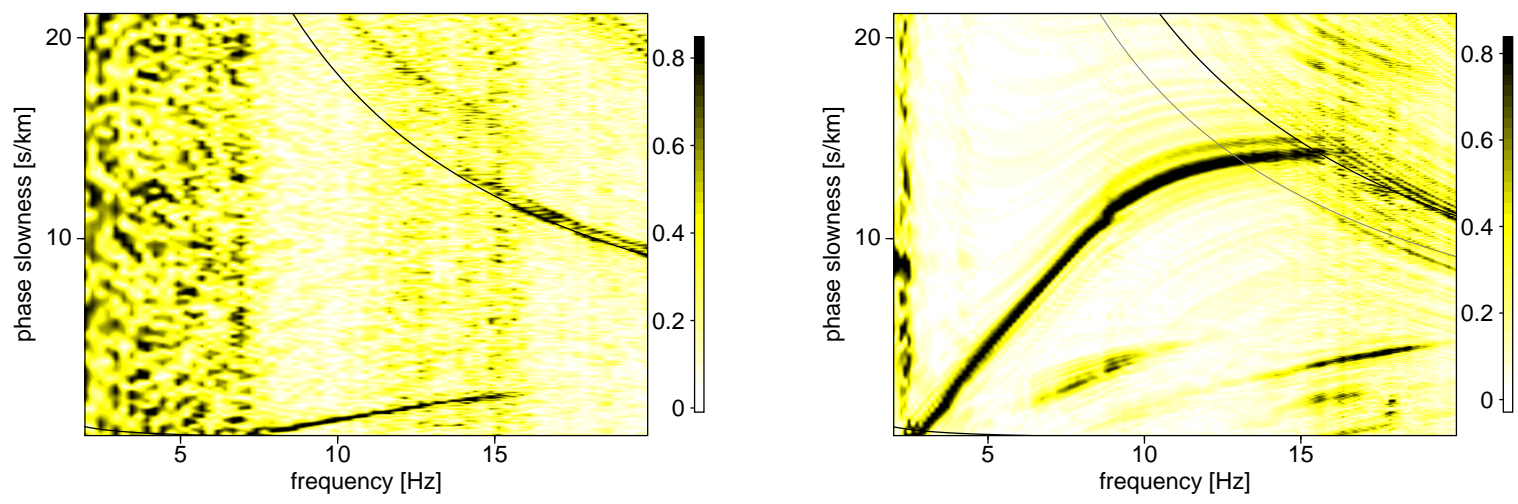

Figure 8.19: Slowness spectra of Al201-P59 recorded at the hydrophone component of OBS 54 from the south-eastern rim of the Arkona Basin (left) compared with the vertical component recording of a planted seismometer in the Tromper Wiek from Al201-P23 


\subsection{Inferring the Shear Wave Velocity by Inversion of the Wavefield Spectra}

The wave field transform using the modified Fourier-Bessel transform was performed on two representative data sets of the study areas. The seismic gathers have been scaled and tapered in offset and time domain prior to the application of the transform. For each inversion run manual interactions after each set of 10 to 20 iterations ensures, that the model is still sensible and the misfit function shows a significant gradient for the free parameters. The normal approach is therefore to start with the inversion of the first arrival times with a set of free parameters combined of all p-wave velocities and layer thickness. The balance factor between the first arrival and spectral coefficient inversion is chosen in favor to the first arrivals. Once the misfit function is stationary, the compressional wave velocities are kept constant, while the shear wave velocity of the layers dominating the dispersion characteristics are selected as free parameters and the balance factor is incremented in order to increase the weighting of the spectral coefficient inversion. The free parameters used are selected from the rating of the partial derivatives for each model parameter. The next set of iterations is always calculated in two stages. Firstly the forward models of the next set of iterations are calculated and the misfit function is plotted for each iteration. If the misfit could be reduced and the changes in the model are sensible, the inversion changes are committed and the new model is accepted as the new working model. Otherwise choice of free parameters or the damping factor is altered for the set of iterations. Since the number of free parameters is restricted, the selection of
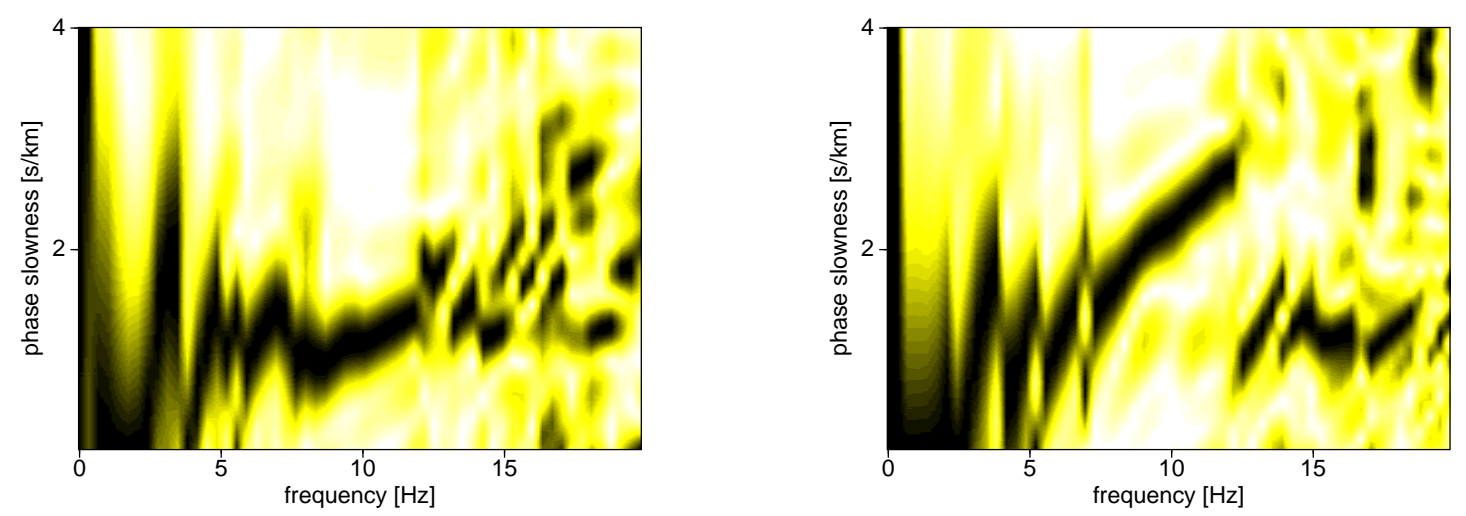

Figure 8.20: The wave spectra for two shots of seismic line Al188-P16 about $2 \mathrm{~km}$ apart indicates lateral variations of the dispersive mode which can be resolved with the towed acquisition system. 


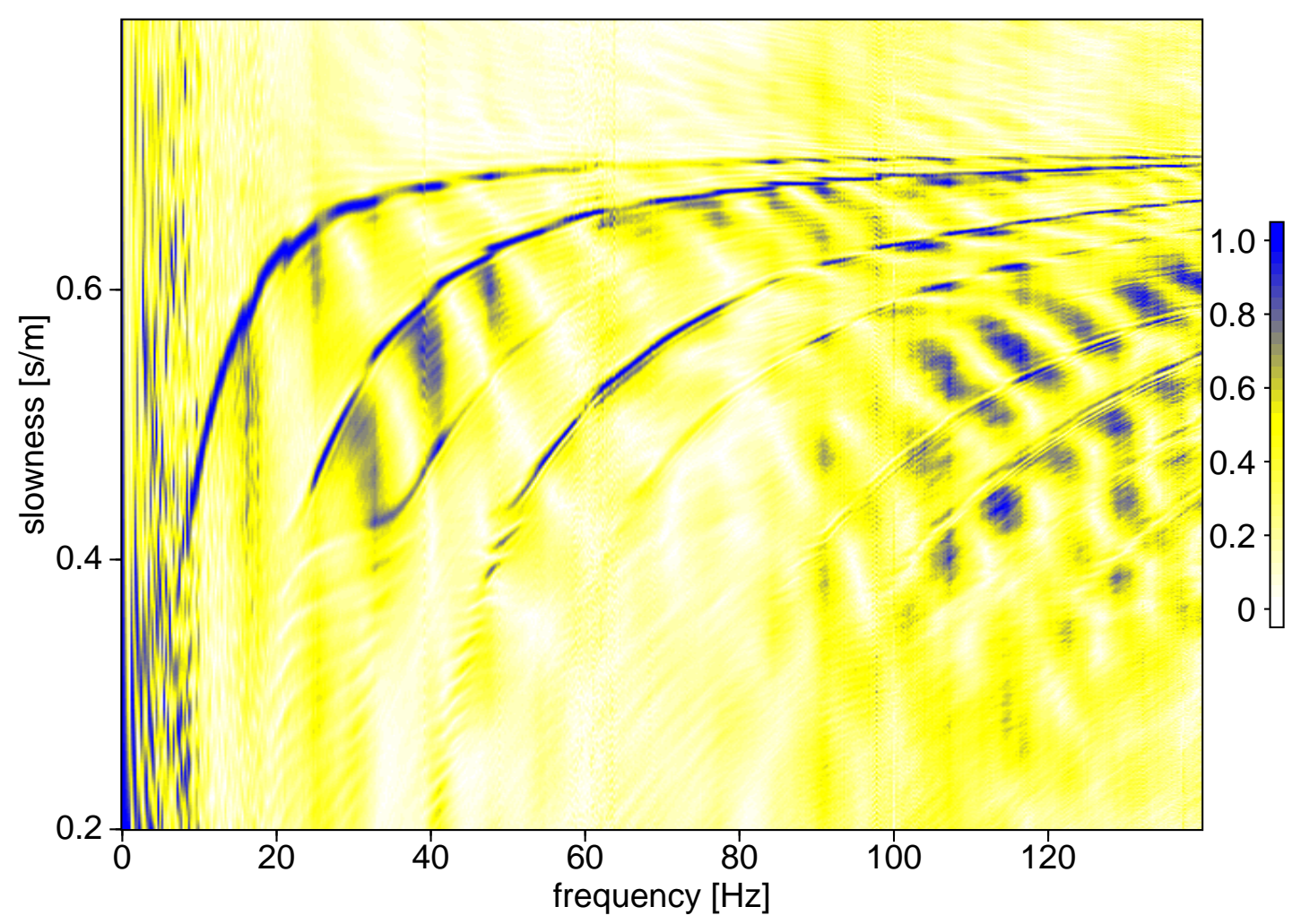

Figure 8.21: The slowness spectrum for the seismic line Al201-P47 (Fig. 8.13 and 8.14) was recorded in the central Arkona Basin on OBS 45. The presence of 6 higher modes within the frequency range from 20 to $120 \mathrm{~Hz}$ corresponds to the guiding influence of the water column. Note the variation of amplitudes of the individual modes as well as the "feathering" below slowness values $0.4 \mathrm{~s} / \mathrm{km}$ for the second and third higher modes. 
free parameters must be checked several times in order to ensure, that the misfit function of the final model is stationary with respect to all significant parameters of the model.
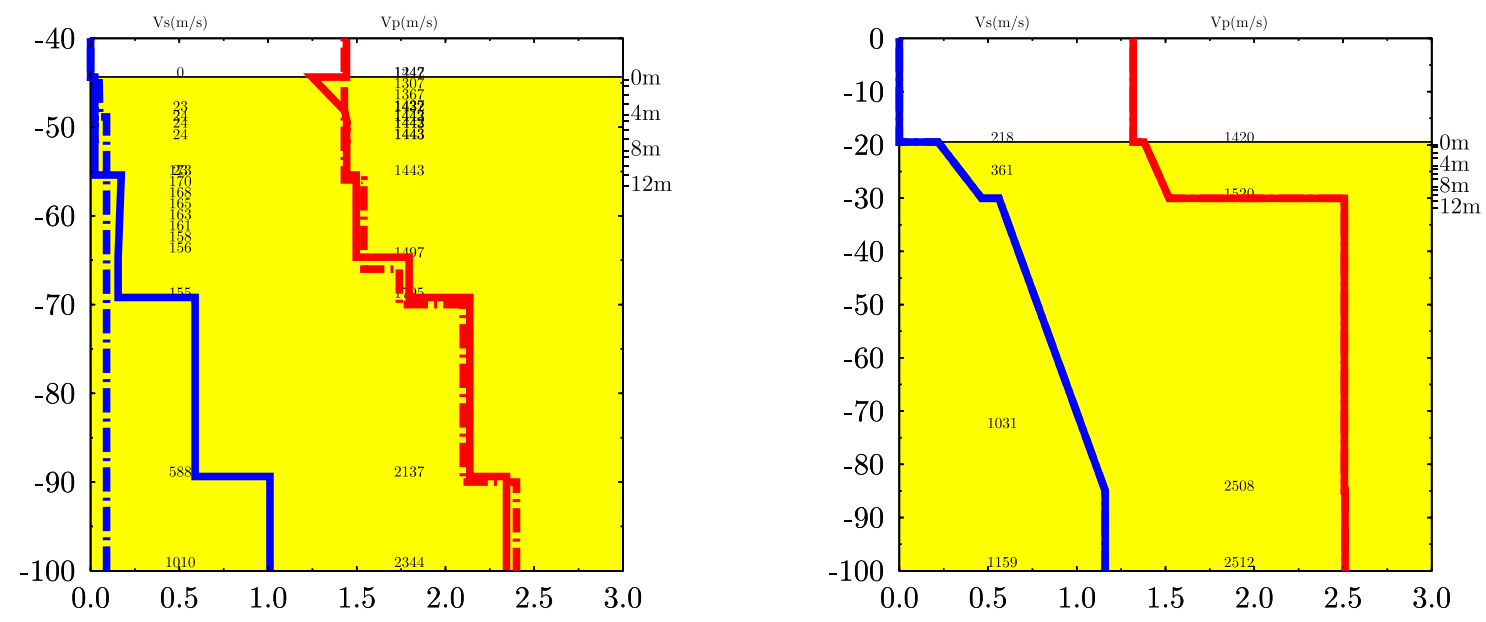

Figure 8.22: Final models of the inversion result

The inversion results for both data sets are presented in fig. 8.22 in form of the final velocity-depth models. Both inversion results were obtained with a log-amplitude scaling applied to both, the measured and synthetic spectra. The final model of the acoustic guided wave data from the central Arkona Basin indicated that the sound speed in water is well defined by the data set at $1425-1430 \mathrm{~m} / \mathrm{s}$. Variations of as little as $10 \mathrm{~m} / \mathrm{s}$ increased the misfit by factors of 5-10. The synthetic wave spectra of both models shown in fig. 8.23 have been accepted to sufficiently reproduce the measured spectra of figs. 8.21 and 8.18. The phase information of the synthetic data is without compensation for the source/receiver effects, hence here the phase remains constant within the amplitude maxima. The solid lines denote the position of the amplitude maxima of the measured spectra and coincide with the amplitude maxima of the synthetic data for demonstrating the quality of the fit of the final model.

The other model parameters not presented in fig. 8.22 have little impact on the inversion result but are shown in fig. 8.24 (top row) of the working screen of the inversion. The synthetic first arrival (bottom left) and spectral coefficients (bottom right) for the model are also visible. The overall misfit function $\chi^{2}$ is a balanced combination of the spectral coefficient misfit and the first arrival misfit according to the weighting between the two inversion results. For both data sets the inversion was based on the spectral coefficients only, since traveltime errors of the asymetric geometry remain uncorrected during the traveltime inversion. 

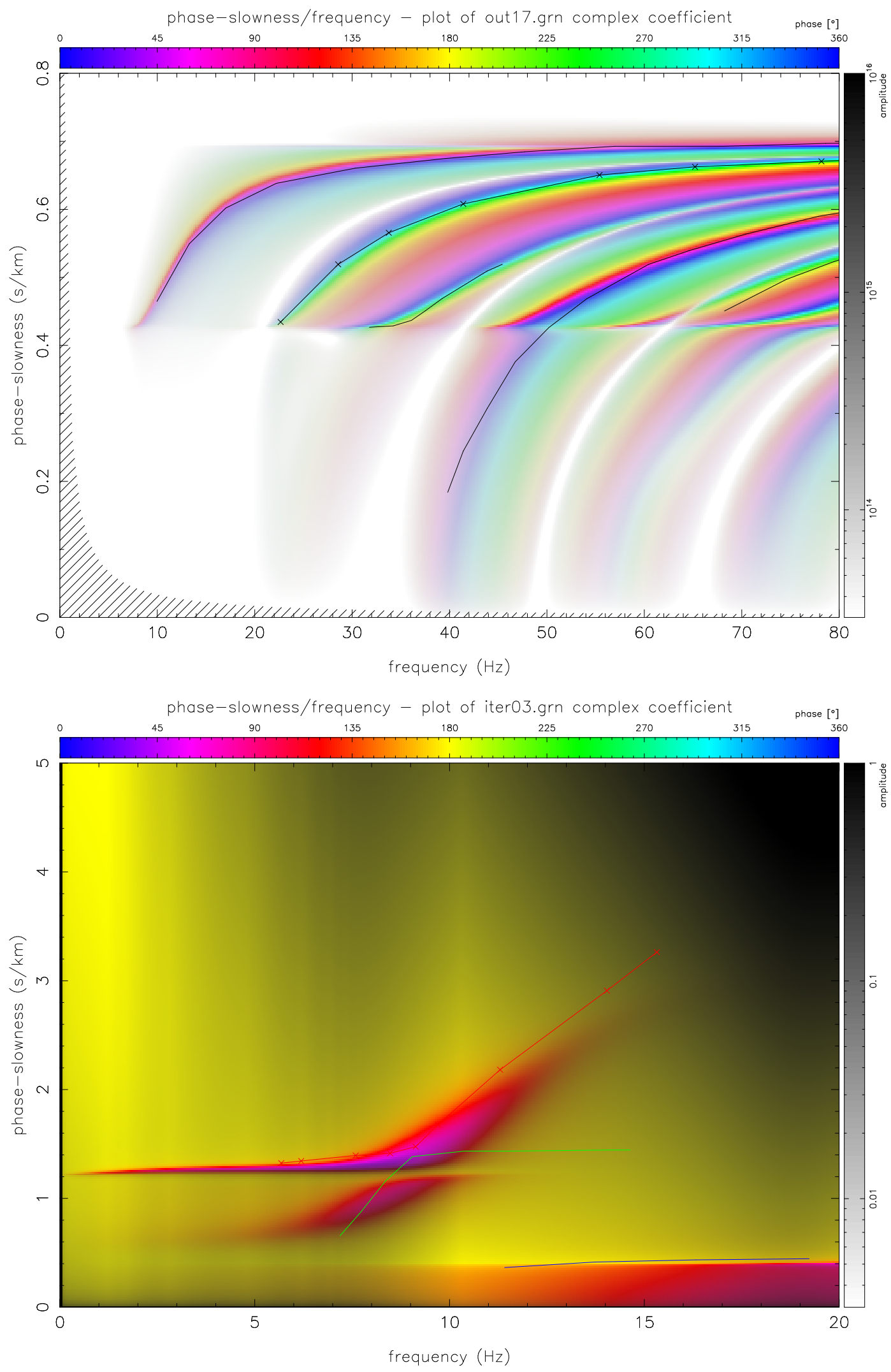

Figure 8.23: Final model and inversion result. 

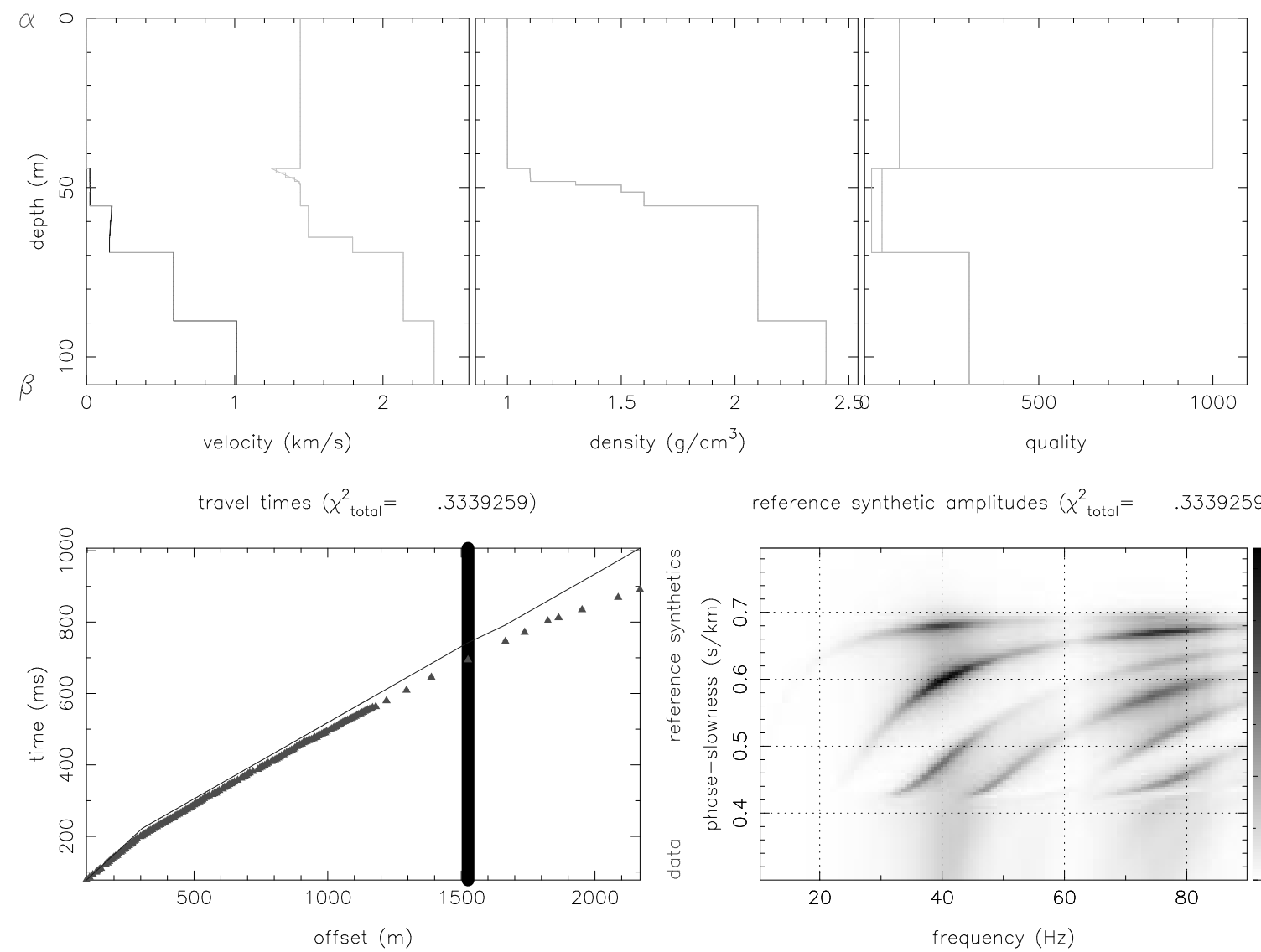

reference synthetic amplitudes $\left(\chi_{\text {total }}^{2}=\quad .3339259\right)$

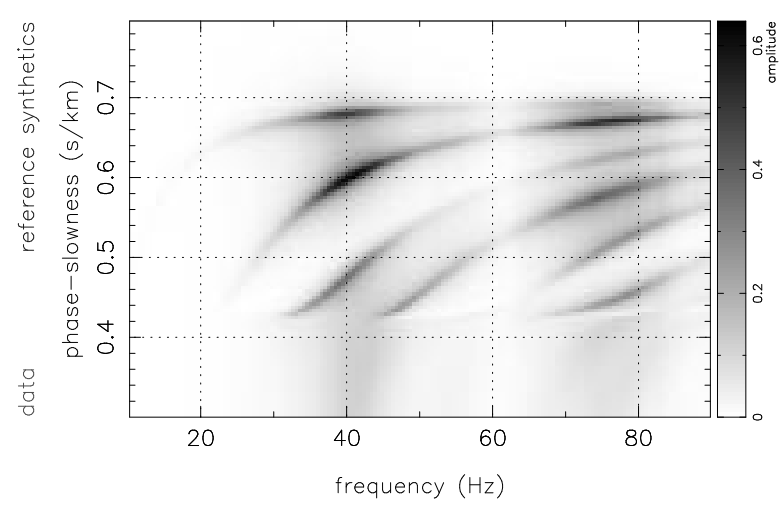

Figure 8.24: Synthetic slowness spectrum of the final model with dispersion curve picks from the measured data. 
The resolution analysis has been performed as described in section 5.3 for $10 \%$ misfit variation. The density and quality factors were fixed, but the seismic velocities in all layers as well as the layer thicknesses were selected as free parameters. In fig. 8.25 all models for all parameter variations are summarized. Velocity gradients have been allowed in the uppermost sedimentary layers. The $10 \%$ variation for the compressional wave velocity and velocity gradient of the first layer indicates the requirement for a steep velocity gradient or a large velocity change in this layer. The inversion result with constant homogeneous layers in the uppermost layer lead to a compressional wave velocity of $1350 \mathrm{~m} / \mathrm{s}$ for this layer with a sudden increase to $1600 \mathrm{~m} / \mathrm{s}$ and a low velocity layer at about $10 \mathrm{~m}$ below the sea bed. The introduction of the velocity gradient into the model parameterization could reduce the tendency towards a low velocity zone at greater depth. Hence, it was assumed that the velocity gradient in the uppermost layer is more realistic and indicated by the measured data.

The shear wave velocity of the uppermost sediment layer is not as well determined. The resolution analysis indicates that positive and negative velocity gradients would be supported equally well. Therefore I conclude that the shear wave velocity in this layer is not well determined, but is only restricted to be within $5-150 \mathrm{~m} / \mathrm{s}$. However, during several inversion runs the tendency of velocity changes in the shear wave velocity of the uppermost $20 \mathrm{~m}$ was towards very low shear wave velocities ( $3-20 \mathrm{~m} / \mathrm{s}$ ) or to no change at all with values remaining at the velocity of the starting model of $90 \mathrm{~m} / \mathrm{s}$. A start model with shear wave velocities exceeding $150 \mathrm{~m} / \mathrm{s}$ in the uppermost sedimentary layer yielded a shear wave velocity profile with very high velocity at the sea bed (exceeding $400 \mathrm{~m} / \mathrm{s}$ ) but a strong negative velocity gradient leading to velocities less than $10 \mathrm{~m} / \mathrm{s}$ at $20 \mathrm{~m}$ depth below sea bed. While this model is in contrast to the expectations as well as to the results of the sediment-physical investigations, this indicates that the shear wave velocity is well constrained for the depth interval from 10

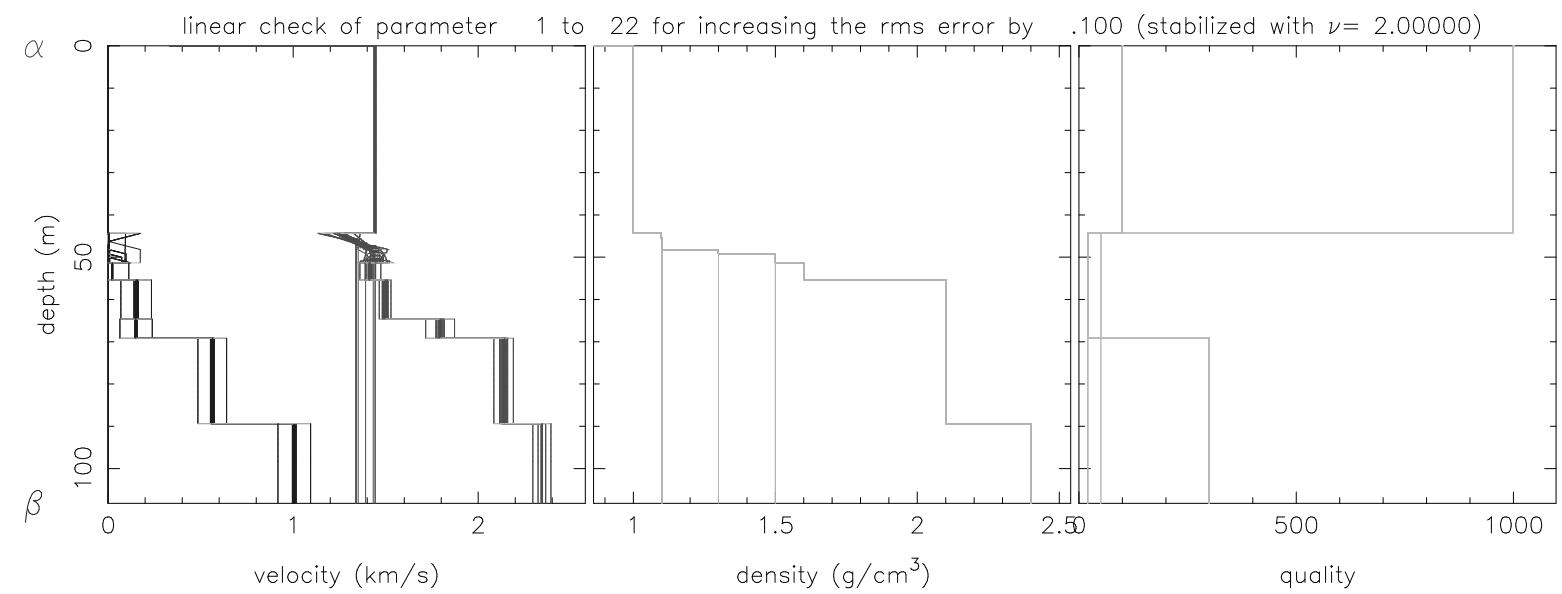

Figure 8.25: Resolution analysis results for all model velocities and layer thicknesses. 
- $30 \mathrm{~m}$ below sea bed, but it is ambiguous directly at the sea bed. The interpretation of the resolution analysis also indicates that the shear wave velocity in the second sedimentary layer is better resolved than the other layer velocities.

\subsection{Conclusions}

The potential of determining lateral variations of dispersive seismic waves with a deep-tow streamer acquisition system is very much depending on the sub-surface structure of the study area. The first acquisition tests indicated that the depth control can be improved with the application of additional depressor weights to the system. Furthermore, this could enable to alter the offset range towards closer offsets. The shot sections recorded with the $200 \mathrm{~m}$ long active section indicated, that the near-offset range from 80 to $180 \mathrm{~m}$ would be of interest in the south-eastern rim study area.

It was confirmed, that the presence of very soft sediments in the central Arkona Basin hinder the measurement of dispersive interface waves. However, very distinct acoustic guided modes have been observed in the OBS recording from this area. Constraints on the shear wave velocity profile are in agreement to previous results from in-situ probing and sediment coring. However, the resolution of the inversion with respect to shear wave velocities is not satisfactory in terms of the determination of the shear modulus of the shallow marine sediments.

Despite of the lack of absolute quantification, an indication on the strength of the sea floor can be derived from acoustic guided waves, when no other wave types are present. The lateral variability of sea floor stability of hard bottom sediments could potentially be determined with a towed acquisition unit, if a priory knowledge of the site is available. 


\section{Part III}

\section{Discussion of Results}





\section{Chapter 9}

\section{Results, Discussion and Conclusions}

\subsection{Acquisition of dispersive waves}

Two dispersive wave types, the Scholte wave and guided acoustic waves, have been acquired and analysed. The proposed methods include the application of a full wave field transform of the measured seismic data to visualize and analyse the seismic wavefield in the frequency-slowness domain. This implies certain restrictions on the data acquisition.

The two types of seismic experiments, the stationary-receiver method as well as the towed-acquisition method can be used to measure dispersive seismic waves in certain conditions. These conditions relate to the sub-surface properties and the type of dispersive waves to be observed and need to be individually checked for each survey area.

The investigations presented in the second part of my thesis show both, potentials as well as limitations of the methods to infer information on seismic properties from dispersive seismic waves for shallow marine sediments. An overview of the seismic wave types observed at the various sites is summarized in table 9.1. The results from the experiments in the Kiel Bay

\begin{tabular}{|c|c|c|c|c|}
\hline Location & $\begin{array}{c}\text { Observed } \\
\text { wave type }\end{array}$ & Acquisition & Sediment type & $\begin{array}{c}\text { Obtained shear } \\
\text { wave velocity }\end{array}$ \\
\hline \hline Kiel Bay & Scholte wave & OBS & glacial till & $260-400 \mathrm{~m} / \mathrm{s}$ \\
\hline Tromper Wik & Scholte wave & OBS & sand & $70-450 \mathrm{~m} / \mathrm{s}$ \\
\hline Arkona Basin II & $\begin{array}{c}\text { Scholte wave } \\
\text { (or Stoneley ?) }\end{array}$ & $\begin{array}{c}\text { OBS }+ \\
\text { Streamer }\end{array}$ & glacial till/chalk & $300-400 \mathrm{~m} / \mathrm{s}$ \\
\hline Arkona Basin I & Acoustic guided & OBH & soft mud & $10-50 \mathrm{~m} / \mathrm{s}$ \\
\hline Laptev Sea & Acoustic guided & Streamer & thawed permafrost & $\approx 700 \mathrm{~m} / \mathrm{s}$ \\
\hline
\end{tabular}

Table 9.1: Observed seismic wave types presented in the case studies in part II. 
study area demonstrate that dispersive Scholte waves yield information on the sub-surface properties at site A of the study area. Furthermore the Baltic Sea experiments could show the advantage of three component seismometer recordings in order to investigate the particle motion as well as the superior data quality in case of good receiver coupling. Two other investigation sites in the Kiel Bay study area indicated the limitations of the simple method of shear wave velocity inversion based on dispersion curve fitting due to the complex patterns in the observed spectra.

The limitations encountered for complex wavefield spectra can be overcome by inversion of the complex spectral coefficients of the wavefield. The limitations of acquiring dispersive interface waves at sites with very soft surfical sediments remain problematic with the survey configurations currently available due to the required dense sampling of the wavefield. However, the experiments in the central Arkona Basin indicated the possibility to obtain structural information on sediment properties despite of the presence of very soft surface sediments. These were obtained from dispersive acoustic guided waves observed in the OBH recordings. This wave type was also observed on the long offset streamer recordings of the Laptev Sea data set. Information on the thickness of the thawn sedimments as well as on the shear wave velocity of sediments and the permafrost was obtained. This gives rise to treat these types of dispersive waves separately in terms of acquisition and inversion methods.

Examples from different marine environments have been investigated within the case studies presented in the second part of the thesis. The variation in acquisition configuration for the different marine sediment structures and the related properties are summarized in table 9.2 for both the interface waves as well as the acoustic guided waves. The acquisition parameters (offst range and spacing) given in brackets correspond to the parameters used in the seismic experiments presented in the case studies. The required parameters derived therefrom are based on the experience of the data analysis of these data sets. The seismic velocities and frequency range refer to the inversion results and observations from the measured data.

Marine seismic interface waves of the Scholte wave type can be observed if the subsurface shear wave velocity is sufficiently high, e.g. exceeding $60 \mathrm{~m} / \mathrm{s}$ as encountered in the experiments in the Tromper Wik. The quantification of the limit depends on the spatial and temporal sampling as well as the frequency range of observation. Here, the stationaryreceiver experiment faces limitations on the shot spacing related to the minimum ship speed to maintain manouverability. This limitation implies that the measurement of Scholte waves is not feasible if the shear wave velocity of the sea floor sediments is below a certain level, which is determined by the frequency and velocity of the Scholte wave and the maximum distance of observation (offset) (cf. Fig. 4.3).

The repitition of the profiles in several runs is one possible solution to enhance the spatial sampling, but requires the very accurate absolute postition of the source to a few tens of 
centimeters which was not achievable at present.

Acoustic guided waves could be observed on long offset streamer and OBH recordings in the shallow $(<50 \mathrm{~m})$ water conditions of the Laptev Sea and the Arkona Basin. The dispersion characteristic observed for this wave type is sensitive to the water depth and speed of sound in water, the sediment density and the compressional as well as the shear wave velocity of the sediment. From the inversion of acoustic guided waves information on the shear wave velocity structure could be obtained at a site, where the measurement of marine seismic interface waves could not be observed. The resolution of the inversion result with respect to the shear wave velocity is best at a depth of 10-20 m below the sea floor for the profile investigated.

The dispersion of Scholte waves, in contrast, is dominantly sensitive to shear wave velocity variations. All other elastic parameters are one or several orders of magnitudes less sensititve.

The navigation system used on the research vessel used to obtain positioning data determines the accuracy of the acquisition configuration in terms of the source-receiver distance used in the data analysis. For the stationary-receiver methods this requires to measure the absolute position of source and receiver. It could be shown that the analysis method is sensitive to errors in the source receiver geometry, if the seismic wave field is determined from small offset recordings below $100 \mathrm{~m}$. The error in the receiver position of up to $+-60 \mathrm{~m}$ in cross-line direction could be compensated by amplitude scaling in favour to the far offset traces or by

\begin{tabular}{|l|c|c|c|c|}
\hline Sediment type & $\begin{array}{c}\text { offset } \\
\text { range }[\mathrm{m}]\end{array}$ & $\begin{array}{c}\text { shot/receiver } \\
\text { spacing }[\mathrm{m}]\end{array}$ & $\begin{array}{c}\text { seismic velocities } \\
{[\mathrm{m} / \mathrm{s}]}\end{array}$ & $\begin{array}{c}\text { frequency } \\
{[\mathrm{Hz}]}\end{array}$ \\
\hline \hline $\begin{array}{l}\text { submarine } \\
\text { permafrost }\end{array}$ & $\begin{array}{c}300+; 800+ \\
(500 ; 3100)\end{array}$ & $\begin{array}{c}<12.5 ; 10-15 \\
(12.4 \mathrm{~m})\end{array}$ & $\begin{array}{c}300 \text { (thawed) } \\
900-2400\end{array}$ & $3-40 ; 15-120$ \\
\hline $\begin{array}{l}\text { boulder clay } \\
\text { glacial till }\end{array}$ & $\begin{array}{c}200+; 800+ \\
(800 ;-)\end{array}$ & $\begin{array}{c}8 ; 10-15 \\
(5-8 \mathrm{~m} ;-)\end{array}$ & $200-500$ & $4-25 ; 10-120$ \\
\hline $\begin{array}{l}\text { silty sands } \\
\text { sandy silts }\end{array}$ & $\begin{array}{c}200+; 800+ \\
(800 ;-)\end{array}$ & $\begin{array}{c}5 ; 10-15 \\
(5 \mathrm{~m} ;-)\end{array}$ & $150-400$ & $2-20 ; 10-120$ \\
\hline $\begin{array}{l}\text { mud } \\
\text { soft clay }\end{array}$ & $\begin{array}{c}? ; 800+ \\
(-; 1600)\end{array}$ & $\begin{array}{c}0.1-0.5 ; 10-15 \\
(5-8 \mathrm{~m} ; 5 \mathrm{~m})\end{array}$ & $10-50$ & $0.1-3 ; 10-140$ \\
\hline
\end{tabular}

Table 9.2: Acquisition parameters required (used) for different sediment and wave types (interface ; guided). The variation of acquisition parameters is much higher for the interface waves than for guided waves. The required shot/receiver spacing render Scholte (interface) wave measurements in soft clay or mud unfeasible. 
discarding seismograms recorded at distances less than $100 \mathrm{~m}$. More important is the change in relative position of the seismograms within one section. This must be determined within a few meters.

The sound speed and depth of water were determined to an accuracy of $0.5 \%$ and less than $3 \%$, respectively, from the inversion of acoustic guided waves in the central Arkona Basin, if the accuracy of the configuration geometry is acceptable. Unfortunately the sound speed of water has not been measured independently since this additional data could be used to cross check the accuracy of the navigational data and the resulting acquisition geometry. In general it is easier to determine the water depth from echo sounder data and the sound speed from CTD measurements, than to accurately determine the position of the OBS and the towed source. Hence, the high sensitivity of the acoustic guided wave to water depth and compressional wave velocity in water could be used as additional information for the determination of the source-receiver geometry.

A towed acquisition system comprised of a deep-towed streamer of $200 \mathrm{~m}$ active length and an airgun source was capable to measure interface wave energy likely to be related to Stoneley wave propagation in the glacial till or chalk layers very close to the surface at the south-east rim of the Arkona Basin. The lateral variation of the interface mode was observed in the data. The frequency range of observation was not sufficient for a stand-alone inversion of the measured wavefield spectra from the streamer data. Yet, this could be compensated by using a starting model obtained from the inversion of a stationary-receiver experiment of the same area.

\subsection{Inversion of dispersive waves}

Two inversion methods to infer sub-bottom properties from dispersive seismic waves have been investigated. The first method is an inversion of dispersion curves to derive shear wave velocity variations in a layered medium. A full wavefield forward algorithm is used to obtain synthetic wave spectra for the given source and receiver configuration. Therefore the limitation to and identification of the fundamental Scholte mode is not required. Separation and identification of the fundamental mode is conventionally required in the SASW or equivalent methods, this does not apply to the proposed method of dispersion curve inversion. Just the requirement of the dispersion curves to be continuous and countable remains. The method was successfully applied at one of three different geological sites in the Kiel Bay, Baltic Sea. The inversion results are in good agreement with the expected values based on previous investigations.

The limitations for more complex wave fields gave rise to the application of the second inversion method investigated in this thesis. The inversion of the wavefield spectra was previ- 
ously applied to Rayleigh wave dispersion. The method was adapted to enable the inversion of marine seismic data and applied to acoustic guided waves observed in the Laptev Sea and the Arkona Basin of the Baltic Sea. A correction term related to near-field components of the synthetic seismic wavefield was included in the inversion algorithm for the inversion of shallow marine seismograms. The problems related to near-field components in the synthetic seismograms calculated for the marine environment, but not observed in the measured data, could be solved with this correction.

The source and receiver influence on the seismic wavefield is another issue of concern for the inversion process and should be thought for in the choice of application of both the acquisition and inversion method. The use of common-receiver-gathers to obtain the measured data has the advantage that no variation of receiver coupling has to be compensated, if a stable source signature can be assumed. This is the case for the airgun source as used in the Baltic Sea experiments. Then all source-receiver effects can be summarized in the source function which is included as an inversion parameter and used for the computation of synthetic wavefield spectra.

The amplitudes and phases of the complex spectral coefficients can be inverted using the proposed inversion of full wavefield spectra, if the influence of the source and receiver is either known or constant and determinable as a free inversion parameter. Here, also, it is favorable to use either common-receiver gathers or receiver gathers with constant receiver effects for all receivers. This assumption is valid for a hydrophone array towed in the water column and the inversion of dispersive seismic waves of deep-tow streamer measurements is feasible. This could be shown in the case of hard surfical sediments encountered in the Siberian Laptev Sea as presented in the second case study. Unfortunately the evidence could not be presented for the soft marine sediments of the central Arkona Basin. Possible causes are the towing of the streamer at intermediate depth, about $15 \mathrm{~m}$ above the sea floor. It is likely that the dispersive interface wave is decayed at this distance from the interface if it was excited at all. At the south-eastern rim study area with comparably hard bottom sediments the successful inversion from deep-tow streamer data was not possible without requiring the a priori knowledge of OBS measurements and geological information. However, this could be due to failures of the acquisition system the requirements of the acquisition parameters in terms of offset range and spatial sampling could not be met. The lack of numerous traces causes artifacts in the wavefield transform. The synthetic spectral coefficients are independent of the lateral distribution of the receivers but assume that the seismic wavefield has been adequately sampled. Hence, the artifacts in the spectral coefficients of the observed seismic wavefield produced by the wavefield transform can not be treated adequately during the inversion process and must cause unresolvable residues or mis-interpretation of the data. 


\subsection{Conclusions}

Scholte waves as well as acoustic guided waves can be excited in shallow marine environments using a surface towed airgun. The successful registration of Scholte waves depends strongly on the sub-surface material properties. Data examples for sediments with shear wave velocities exceeding $60 \mathrm{~m} / \mathrm{s}$ could be obtained. Both the stationary-receiver method as well as a towed acquisition system can be used to measure the lateral variation of the dispersion characteristics of the Scholte wave. Limitations for the acquisition of Scholte waves remain for very soft marine sediments with shear wave velocities lower than $50 \mathrm{~m} / \mathrm{s}$ due to the required dense spatial sampling and the low frequency range of interest for these conditions. Similar restrictions in terms of the frequency range should be envisioned for the application in deeper waters, since the frequency range of interest is affected by the water depth. However, for shallow marine sediments exceeding shear wave velocities of $60 \mathrm{~m} / \mathrm{s}$ sufficient Scholte wave energy could be excited with a surface towed airgun and the shear wave velocity could be successfully inverted from dispersive seismic wavefields recorded at or near the sea floor.

Acoustic guided waves recorded with a long streamer in the Laptev Sea and with a stationary receiver in the central Arkona Basin could be used to infer sub-surface properties including the shear wave velocity by inversion of the full wavefield spectra. Since this wave type is sensitive to compressional wave velocity and density as well as the shear wave velocity, the ambiguities have to be compensated by additional constraints. However, shear wave velocity information was obtained from this wave type at the central Arkona Basin where no seismic interface wave could be recorded. This wave type is generally known to require a hard contrast at the sea floor, yet the acoustic guided wave could be observed and analyzed in the central Arkona Basin despite of the presence of a 10 to $12 \mathrm{~m}$ thick layer of soft sediments above stiffer materials. However, the sensitivity of the shear wave velocity information is low for the soft sediment layer in this specific data set.

The advantage of using full wavefield solutions in the forward computations of the inversion procedures was investigated for the inversion of dispersion curves as well as the full wavefield spectra. The synthetic seismic wavefield accounts for the source and receiver geometry and therefore the information on the excitation of specific modes enables to extract additional information on the sub-surface from the measured data. In case of the inversion of the spectral coefficients of the full seismic wavefield even the full amplitude information can be used to derive sub-bottom parameters.

While it was possible to use dispersion curve fitting for multi-modal seismic wavefields this method is limited to dispersion curves of a countinous and countable nature. However, even a simple structure can produce complex dispersion relations, which was demonstrated by examples in the case studies in part two of the thesis. Algorithms based on dispersion curve fitting will fail in these cases (e.g. site $\mathrm{C}$ in Kiel Bay).

A proposition is to use a hybrid method to derive weighted dispersion curves from the 
data, which are then compared with the synthetic amplitude spectra of seismic models. This can overcome many of the problems of dispersion curve inversion methods, namely the requirement on identification and separation of modes. The problems due to noise or source and receiver specific influences on the measured data are reduced since the amplitudes of the measured seismic wavefield are not inverted directly, but suitable weighting of the synthetic and measured data is applied.

It is therefore possible to include relative amplitudes between modes without further requirement on the knowledge of source spectrum or true amplitudes. This would also be an achievable and useful extension of the much more simple dispersion curve inversion procedures. Both of the presented inversion methods aim to simplify difficulties arising in full wave form inversion, but still include the information of the amplitude and phase spectrum of the seismic wavefield in order to obtain additional information on the properties of the marine sediments.

\subsection{Outlook}

The general applicability to derive shear wave velocities from the measurement of dispersive seismic waves has to be considered with respect to several factors. The application of a surveying method to determine shear wave velocities in shallow marine environments in confined areas ( e.g. harbors) is of special interest for marine geotechnical applications. Due to the required offset ranges to adequately determine the dispersive seismic wave field the method doesn't seem to be applicable in such confined study areas. Furthermore, the dependence of the survey configuration on the target property itself (the shear wave velocity) is problematic in cases when no a priori knowledge is available. This restricts the applicability to open areas and to survey targets, which can be determined with a survey configuration as summarized in table 9.2. However, this could be of interest if the target of investigation is to find locations with shear resistance and shear wave velocities exceeding the level determinable with a given acquisition configuration. In large areas of investigation this could be achieved with a towed acquisition configuration, once a background model is determined from a more detailed seismic experiment at one location. As presented in the Arkona Basin case study the dispersive interface wave could be measured only in places, when a hard subsurface structure was present. From the dispersive seismic waves we could specify that the shear wave velocity must exceed $400 \mathrm{~m} / \mathrm{s}$ in this layer. Hence, we can determine areas, which are characterized by hard sediments at shallow sub-surface depth.

Furthermore the future investigations should aim to conduct of combined surveys in order to obtain a priori information required for the purpose of a joint data analysis. This should include high-resolution seismic surveys, preferably with a multi-channel recording in order to obtain velocity information from refraction and reflection seismic data analysis and to per- 
form amplitude versus offset investigations. These could provide additional constraints on density and compressional velocity as well as shear wave velocity and depth of interfaces at a certain extend (Riedel \& Theilen, 2001). The calculation of reflection coefficients from multiples in high-resolution seismic profiles and the penetration and reflection strength of echo sounder data could also provide additional information (Mathys, 2003; Klein, 1998; Riedel, 1998). The investigation of converted waves could be included in the inversion process. The reflection coefficient of converted shear waves in combination with predicted travel times could be used as additional constraints for the inversion. This would require to pick reflection events which are likely to be related to converted waves. Bussat investigated the potential to identify converted wave arrivals in shallow marine environments (Bussat, 2001).

This integrative approach to combine the information of several independent analysis method has a high potential to reduce ambiguities and to stabilize the inversion with true information on the sub-surface. This motivates to use the concept of Parker (1994) by developing an inversion with the target to find the most simple model of the sub-surface that is not in disagreement with the observations. The additional constraints of this inversion should be, that the model is not in contradiction to any of the data sets. A joint inversion algorithm with modular components for several data sets would be favorable. 


\section{References}

AKI, K., \& RichardS, P.G. 2002. Quantitative seismology. University Science Books, Sausalito, California.

ANONYMOUS. 1985. The International System of Units (SI) in oceanography/ Report of IAPSO Working Group on Symbols, Units and Nomenclature in Physical Oceanography (SUN). Tech. rept. 32. UNESCO.

Ayres, A., \& Theilen, F. 1999. Relationship between P- and S-wave velocities and geological properties of near-surface sediments of the continental slope of the Barents Sea. Geophys. Prosp., 47(4), 431-441.

BEATY, K, \& SCHMitT, D. 2000. A study of near-surface seasonal variability using rayleigh wave dispersion. Ann. Mtg. of SEG, Ex. Abstr., 70, 1323-1326.

Bohlen, T., Klein, G., Duveneck, E., Milkereit, B., \& Franke, D. 1999. Analysis of dispersive seismic surface waves in submarine permafrost. Pages talk 6-37 of: EAGE 61th Conference and Technical Exhibition, Expanded Abstracts.

Bohlen, T., Kugler, S., Klein, G., \& Theilen, F. 2003. 1.5-D Inversion of Lateral Variation of Scholte Wave Dispersion. Geophysics, submitted.

Bohlen, Thomas. 1998. Viskoelastische FD-Modellieung seismischer Wellen zur Interpretation gemessener Seismogramme. Dissertation, Kiel University.

Bourbie, T., Coussy, O., \& Zinszner, B. 1987. Acoustics of Porous Media. Huston: Gulf Publishing Co.

Buchen, P., \& BEN-HAdOR, R. 1996. Free-mode surface-wave computations. Geophys. Journ. Int., 124, 869-887.

Bussat, S. 2001. Konvertierte Scherwellen bei weichen Meeresböden. Entwicklung eines Scherwellenprozessings. Diplomarbeit, Christan-Albrechts-Universität zu Kiel. 
CARcione, J.M., \& Tinivella, U. 2000. Bottom-simulating reflectors: Seismic velocities and AVO-effects. Geophysics, 65(1), 54-67.

D' Arnaud Gerkens, J.C. 1989. Foundation of Exploraiton Geophysics. Amsterdam: Elsevier.

DAVIS. 1996. Geophysical Ground-truthing Experiments in Eckernförde Bay. Geo-Marine Letters, 16(3), 160-166.

Deslile, G. 1998. Temporal Variablilty of Subsea Permafrost and Gas Hydrate Occurances as Function of Climate Change in the Laptev Sea, Siberia. Pages 221-225 of: Polarforschung, vol. 68 .

Dullien, F.A.L. 1992. Porous Media: Fluid Transport and Pore Structure. San Diego: Acad. Press.

DVORKIN, J., \& NUR, A. 1993. Dynamic poroelasticity: A unified model with the squirt and the Biot mechanisms. Geophysics, 58, 524-533.

Dziewonski, A., Block, S., \& Landisman, M. 1969. A technique for analysis of transient seismic signals. Bull. Seism. Soc. Am., 59, 427-444.

Edelman, I.YA. 2002. Surface Waves in Porous Media. Izvestiya, Physics of the Solid Earth, 38(1), 72-89.

ENDLER, R. 1989. Akustische Eigenschaften des seebodennahen Schlicks im Arkonabecken. Dissertation, Institut für Meereskund Warnemünde.

FORBRIGER, TH. 2001. Inversion flachseismischer Wellenfeldspektren. Dissertation, Stuttgart University.

FORBRIGER, TH. 2003a. Inversion of shallow-seismic wavefields. Part 1: Wavefield transformation. Geophys. Journ. Int., 153(3), 719-734.

FORBRIGER, TH. 2003b. Inversion of shallow-seismic wavefields. Part 2: Inferrring subsurface properties from wavefield transforms. Geophys. Journ. Int., 153(3), 735-752.

Franke, D., Hinz, K., \& Oncken, O. 2001. The Laptev Sea Rift. Marine and Petroleum Geology, 18, 1083-1127.

Friederich, W., \& Dalkolmo, J. 1995. Complete synthetic seismograms for a spherically symmetric earth by a numerical computation of Green's function in the frequency domain. Geophysical Journal International, 122, 537-550. 
FuCHS, K., \& MÜLlER, G. 1971. Computation of synthetic seismograms with the reflectivity method and comparison with observations. Geophys. J. R. astron. Soc., 23(4), 417-433.

GAZETAS, G. 1982. Vibrational characteristics of soil deposits with variable velocity. $J$. Num: Anal. Meth. Geomech., 6, 1-20.

Gettrust, J., Wood, W., Lindvall, D., Chapman, R., Walia, R., Hannay, D., Spence, G., Louden, K., MacDonals, R., \& Hyndman, R.D. 1999. New Seismic Study of Deep Sea Gas Hydrates Results in Greatly Improved Resolution. EOS, (9)(September).

GIMPEL, PETER. 1987. Marine flachseismische Untersuchungen in der Kieler Bucht unter besonderer Berücksichtigung von Scherwellenmessungen. Dissertation, Kiel University.

Glangeaud, F., Mari, J-L., Lacoume, J-L., Mars, J., \& Nardin, M. 1999. Dispersive seismic waves in geophysics. Europ. Journ. of Env. and Eng. Geophys., 3, 265-306.

Grace, A. 1994. Otimization Toolbox for use with MATLAB. The MathWorks Inc.

Haskell, N. 1953. The dispersion of surface waves on multilayered media. Bull. Seism. Soc. Am., 43, 17-34.

Hinz, K., Delisle, G., Cramer, B., Franke, D., Fieguth, U., Lindemann, F., Neben, S., Tostmann, H., \& Zeibig, M. 1997. Marine Seismic Measurements and Geoscientific Studies on the shelf and slope of the Laptev Sea \& East Siberian Sea/Arctic. Tech. rept. BGR.

KJartansson, E. 1979. Constant Q-Wave propagation and attenuation. Journ. Geophys. Res., 84(B9), 4737-4748.

KLeIN, G. 1998. Sediment Properties from Sub-bottom Profiler data. Diplomarbeit, School of Ocean Sciences, University of Wales, Bangor.

Klein, G., Bohlen, T., Theilen, F., \& Milkereit, B. 2000. OBH/OBS versus OBS Registration for measuring dispersive marine Scholte waves. Page poster of: EAGE 62th Conference and Technical Exhibition, Expanded Abstracts.

Krone, R. 1997. Sismique onde S en faible profondeur d'eau: Propriétés de cisaillement des sédiments marins superficiels par inversion simultanée de la dispersion desondes de Love et de Scholte. These de Doctorat de l'Université de Bretagne Occidentale. 
KUGLER, S. 2001. Bestimmung der Scherwellengeschwindigkeit flacher mariner Sedimente aus der Bestimmung von Scholtewellen. Diplomarbeit, Christian-Albrechts-Universität zu Kiel.

LANGE, G. 1997. Korrelation sedimentphysikalischer Parameter mit seismischen Messungen. Diplomarbeit, Universität Kiel.

LEMKE, W. 1998. Sedimentation und paläogeographische Entwicklung im westlichen Ostseeraum (Mecklenburger Bucht bis Arkonabecken) vom Ende der Weichselvereisung bis zur Litorinatransgression. Marine Science Reports, 31, 156p.

Luke, B.A., \& Stokoe, K.H. 1998. Application of SASW method underwater. Journ. Geotechn. and Geoenvironm. Eng., 124(6), 523-531.

MAthys, M. 2003. Seismic characterisation and sedimentological investigation of gasrich near surface sediments in Arkona Basin (Baltic Sea). Diplomarbeit, Inst. of Geosciences, University Kiel.

Matthäus, W., Nausch, G., Lass, H.-U., Nagel, K., \& Siegel, H. 2001. Hydrographisch-chemische Zustandseinschätzung der Ostsee 2000. Pages 27-88 of: MAtThäUs, W., \& NAUSCH, G. (eds), The hydrographic-hydrochemical state of the western and central Baltic Sea in 1999/200 and during the 1990s. MSR, vol. 45. IOW.

Matthews, M.C., Hope, V.S., \& Clayton, C.R.I. 1996. The use of surface waves in the determination of ground stiffness profiles. Proc. Instn. Civ. Engrs. Geotech. Eng., 119, 84-95.

Mavko, G., Mukerui, T., \& Dvorkin, J. 1998. The Rock Physics Handbook - Tools for Seismic Analysis in Porous Media. Cambirdge University Press, Cambridge-New York-Melbourne.

McMechan, G.A., \& Yedlin, M.J. 1981. Analysis of dispersive waves by wave field transformation. Geophysics, 46(6), 869-874.

Moros, M. 1999. Spätquartäre sedimentation am Reykjanes Rücken und in der Westlichen Ostsee - Rekonstuktion anhand hochaufösender sedimentphysikalischer Eigenschaften. Dissertation, Ernst-Moritz-Arndt Universität Greifswald.

Moros, M., Lemke, W., Kuijpers, A., Endler, R., Jensen, J.B., Bennike, O., \& GingELE, F. 2002. Regressions and transgressions of the Baltic basin reflected by a new high-resolution deglacial and postglacial lithostratigraphy for Arkona Basin sediments (western Baltic Sea). Boreas. 
Müller, ChF., JÄGer, T., Theilen, F., \& Milkereit, B. 1999. Acquisition, Processing and Interpretation of Marine Data from Single Scatterers. Page P590 of: EAGE, vol. 2.

MUYZERT, E. 2000. Scholte wave velocity inversion for a near surface S-velocity model and PS-statics. In: Ann. Mtg. Soc. Expl. Geophys, Ex. Abstr.

NAZARIAN, S. 1984. In situ determination of elastic moduli of soil deposits and pavement systems by spectral-analysis-of-surface-waves method. Dissertation, University of Texas, Austin, USA.

Nolet, G. 1977. The upper mantle under Western Europe inferred from the dispersion of Rayleigh modes. Journal of Geophysics, 43(1-2), 265-285.

Nolet, G., \& Dorman, L.M. 1996. Waveform analysis of Scholte modes in ocean sediment layers. Geophys. Journ. Int., 125(2), 385-696.

Osler, John C., \& Chapman, DaVid M. F. 1998. Quantifying the interaction of an ocean bottom seismometer with the seabed. Journ. Geophys. Res., 103(B5), 9879-9894.

PARK, C. B., Miller, R. D., \& XiA, J. 1999. Multichannel analysis of surface waves. Geophysics, 64(03), 800-808.

Park, C.B., Miller, R.D., Xia, J., Ivanov, J., Hunter, J.A., Good, R.L., \& Burns, R.A. 2000. Multichannel analysis of underwater surface waves near Vancouver, B.C., Canada. Chap. Expanded Abstracts of: Ann. Internat. Mtg. of. SEG.

PARKER, R. 1994. Geophysical Inverse Theory. Princeton University Press.

Press, F., \& EwIng, W.M. 1952. Surface waves and mantle structure. Geological Society of America Bulletin, 63(12 Part 2), 1356.

RAUCH, D. 1980. Experimental and theoretical studies of seismic interface waves in coastal waters. Pages 307-326 of: Kuperman, W.A., \& Jensen, F.B. (eds), BottomInteracting Ocean Acoustics. NATO Conf. Ser. 4, vol. 5.

RIEDEL, M. 1998. AVO/AVA-Untersuchungen an flach-marinen Sedimenten. Diplomarbeit, Kiel Universitiy.

Riedel, M., \& Theilen, F. 2001. AVO investigations of shallow marine sediments. Geophys. Prosp., 49(2), 198-212.

ROTh, M., \& Hollinger, K. 1999. Inversion of source-generated noise in high resolution seismic data. The Leading Edge, 18(12), 1402-1406. 
SCHNEIDER, C. 1993. Erkundung des Untergrundes von Deponien und Altlasten mit Rayleigh-Oberflächenwellen. Reihe A, Nr. 36, Ruhr Universität Bochum.

SCHÖN, J.H. 1998. Physical properties of rocks: Fundamentals and principles of petrophysics. Handbook of Geophysical Exploration, vol. Band 18. Elsevier Science Ltd., Oxford, 2nd edition.

SCHWAB, F.A., \& KNOPOFF, L. 1972. Fast surface wave and free mode computations. Methods in Computational Physics, 11, 87-180.

SEIDl, D., \& Müller, S. 1977. Seismische Oberflächenwellen. Journ. Geophys., 42, 283-328.

Stokoe, Kenneth-H II, Gauer, R-C, \& Bay, J-A. 1991. Experimental investigation of seismic surface waves in the seafloor. Pages 51-58 of: HoveM, JENS M., RICHARDSon, Michael D., \& Stoll, Robert D. (eds), Shear Waves in Marine Sediments. Kluwer Acad. Publ.

Stoll, R.D., \& BAutista, E.O. 1994b. New tools for studying seafloor geotechnical and geoacoustic properties. JASA, 96(5), 2937-2944.

Theilen, F., Ollier, G., McGee, T., Brussaard, P., De Batist, M., Ori, G., Perini, L., Kögler, F.Chr, Neben, S., \& Omlin, A. 1993. GISP - Geophysical In Situ Probe. Tech. rept. Final Report of the MAST project, Contract number CT90-0057.

Theilen, FR., \& PeCher, I. 1991. Assessment of shear strength of the sea bottom from shear wave velocity measurements on box cores and in-situ. Pages 67-74 of: HovEM, J.M. (ed), Shear waves in marine sediments. Kluwer Academic Publishers.

Thomson, W.T. 1950. Transmission of elastic waves through a stratified solid medium. $J$. Appl. Phys., 21, 89-93.

WANG, R. 1999. A simple orthonormalization method for stable and efficient computations of Green's functions. Bull. Seism. Soc. Am., 89(3), 733-741.

Whitcombe, D.N., Connolly, P.A., Reagan, R.L., \& Redshaw, T.C. 2002. Extended elastic impedance for fluid and lithology prediction. Geophysics, 67(1), 63-67.

Xia, J., Miller, R.D., \& PARK, C.B. 1999. Evaluation of the MASW technique in unconsoliddated sediments. In: Ann. Mtg. of SEG, Exp. Abstr.

XiA, J., Miller, R.D., \& PARK, C.B. 2000. Advantages of calculating shear-wave velocity from surface waves with higher modes. In: Ann. Mtg of SEG, Exp. Abstr. 


\section{List of Figures}

2.1 Models and theories to describe porous media (Schön, 1998) . . . . . . . 18

2.2 The stress-strain relation for an unconfined body. The supscripts $k, l$ represent the components $x, y, z \ldots \ldots \ldots \ldots \ldots \ldots$

2.3 Stress-strain relation for a confined body. The subscripts $1,2,3$ denote the components of principle stresses, which conicide with the components $x, y, z$ in the coordinate system specified. . . . . . . . . . . . . . . 22

2.4 Rheological bodies to describe a viscoelastic material (in Mavko, 1998) . . . 24

2.5 Particle movement for P, SV and SH body waves (top) and the P-SV interface wave (bottom) . . . . . . . . . . . . . . . . . . 25

2.6 Vertical (a) and horizontal (b) component of the normalized particle displacement as a function of depth for the Scholte wave . . . . . . . . . . . . . . . 29

2.7 Schematic diagram for a wave guide . . . . . . . . . . . . . . 31

2.8 Group and phase velocity of the fundamental first and second higher modes of a wave guide (Love waves) normalized by the velocity of the halfspace . . 33

2.9 Frequency ranges of fluid flow processes in porous sediments (in Mavko, 1998) 34

3.1 Comparison of seismogram gather and slowness spectra for full (OBS) and limited (Streamer) offset ranges. . . . . . . . . . . . . . . . . . . . . 43

3.2 Comparison of the wavefield spectra obtained by the application of a frequency domain slant stack (left) and a modified Fourier-Bessel transform (right) of a real data set further discussed in chapter $8 \ldots \ldots$. . . . . . . . . 4

4.1 The variation of dispersion curves with sediment properties for the model given in a) (top left) is shown for variations in shear wave velocity (bottom left) and sediment layer thickness (bottom right). The errorbars of the dispersion curves (top right) represent the amplitude coefficients relating to the obtainable amplitudes for excitation and recording at $15 \mathrm{~m}$ and $20 \mathrm{~m}$ depth corresponding to a deep-towed streamer experiment. 
4.2 The variation of the energy of the eigenfunctions with depth is displayed for discrete frequencies of the fundamental mode (red, amplitudes to the left) and the second mode (blue, amplitudes to the right) in the frequency range from 3 to $15 \mathrm{~Hz}$. The exponental decay of the energy in the water column is more dominant for the high frequencies, hence the frequency band is increased by towing the acquisition system close to the sea bed. Furthermore is the information at higher resolution enhanced if higher modes are included, since these dominate at high frequencies. . . . . . . . . . . . . . . . .

4.3 Lowest resolvable interface wave velocity in a stationary-receiver experiment with speeds from $0.5 \mathrm{~m} / \mathrm{s}$ (lowest line) to $2.5 \mathrm{~m} / \mathrm{s}$ ( $\sim 5 \mathrm{kn}$, top line) and $4 \mathrm{~Hz}$ sensors with respect to the source-receiver distance. . . . . . . . . .

4.4 Data example to demonstrate the effect of aliasing. The amplitude of the dispersion curve is folded to reappear at zero slowness at the frequency where the hyperbolic line(black) meets the signal energy. . . . . . . . . . . . .

5.1 Crossection of the uncorrected (left) synthetic wavefield spectrum at frequency $40 \mathrm{~Hz}$ and the correction term to be applied (right). The real (top) and imaginary (bottom) part of the complex spectral coefficients is plotted with respect to phase slowness at constant frequency. The peaks relate to the fundamental and higher modes of the seismic wavefield.

5.2 Crossections at $40 \mathrm{~Hz}$ for the corrected synthetics and measured spectral coefficients in the representation corresponding to fig. 5.1. . . . . . . . . 70

5.3 Synthetic wavefield spectra with and without the near-field correction applied. 71

6.1 (left page) The bathymetry map of the Baltic Sea shows the locations of the study areas of the Kiel Bay and Arkona Basin case studies presented in chapters 6 and 8 (top). The Laptev Sea study area described in chapter 7 is indicated in the bathymetric map of the Polar Seas (bottom, courtesy of IOBAC, 2003) . . . . . . . . . . . . . . . . . . 77

6.2 Survey configuration in the first seismic experiment at Kiel Bay, Baltic Sea, Sept. 1999 . . . . . . . . . . . . . . . . . . . .

6.3 Location of the two surveys in the Kiel Bay, Baltic Sea. The first Survey (1999) focused on site A, while measurements at A, B and C were obtained

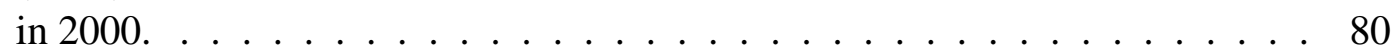

6.4 Location of profiles acquired in the Kiel Bay, Aug. 2000 experiment . . . . . 81

6.5 Common-receiver-gather of the OBS prior and after the application of the low-pass filter at $20 \mathrm{~Hz} \ldots \ldots \ldots$. . . . . . . . . . . . 85

6.6 Comparison of the wavelets (left) and the corresponding frequency spectra (right) from the $\mathrm{OBS}, \mathrm{OBH}$ and $\mathrm{OBC} \ldots \ldots . \ldots . \ldots 86$ 
6.7 Comparison of seismic shot gathers of various ocean bottom sensors . . . . . 87

6.8 The particle displacement for the three components ( $\mathrm{z}=$ vertical and $\mathrm{x}, \mathrm{y}=$ horizontal) of the seismometer recording (OBS) and the hodograms of the particle motion projected in the three planes (from Kugler, 2001) . . . . . . . . . 88

6.9 The results from the wavefield transform into the frequency-slowness domain of the shot gathers shown in fig. 6.7. . . . . . . . . . . . . . . . . 89

6.10 Common-receiver-gathers recorded at site A. The body wave arrivals have been suppressed by low-pass filtering, while the two modes of the Scholte wave energy propagating at low group velocity are very clear (from Kugler,

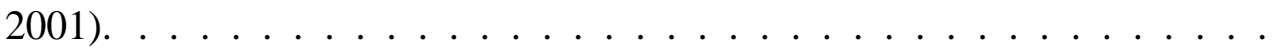

6.11 Inversion results from site A compared with data from previous investigations (from Kugler, 2001) . . . . . . . . . . . . . . . . . . 92

6.12 Resolution analysis for the inversion result for Site A was performed by the "rubber band" test, i.e. by successive variation of the shear wave velocity value in each of the $5 \mathrm{~m}$ thick layers producing an increase in misfit of $10 \%$.

6.13 The common-receiver-gathers from sites B (top) and C (bottom) are not as clear as the corresponding seismic section of site A (from Kugler, 2001). . . . 95

6.14 Synthetic seismogram and the corresponding model possibly resembling the geological situation at site B (from Kugler, 2001) . . . . . . . . . . . . . 96

6.15 The measured p-f-spectrum from site $C$ (right) compared with a synthetic spectrum (center) corresponding to the shear wave velocity model on the right (from Kugler, 2001) . . . . . . . . . . . . . . . . . . . 96

7.1 The bathymetry map of the Laptev Sea shows the large plateau area of the shelf with water depth below $100 \mathrm{~m}$ up to $300 \mathrm{~km}$ and a sudden drop into deep waters. The tectonic setting is indicated by the Gakkel Ridge, i.e. the mid-ocean ridge and boundary between the North American and Eurasien plates, and the mountain ranges on shore. . . . . . . . . . . . . . . 102

7.2 Location of study area and seismic lines. The data example of the multichannel seismic lines have been selected near the locations of the $\mathrm{OBH}$ indicated in the map. . . . . . . . . . . . . . . . . . . . . . . 104

7.3 Acquisition parameter of the 1997 survey in the Laptev Sea conducted by the BGR and SMNG. . . . . . . . . . . . . . . . . . . . . . . . 105

7.4 In the example shot gather from the reflection seismic line 21 the refracted arrivals with several reverberations have a velocity exceeding $3 \mathrm{~km} / \mathrm{s}$ related to shallow depth. This indicates the presence of submarine permafrost. The acoustic wave exhibits dispersion which is more clear in the time reduced zoom in fig. 7.5 and marked by the box. The amplitudes are scaled with

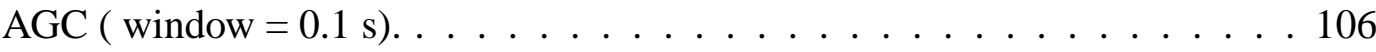


7.5 The time reduced section of the shot gather in fig. 7.4 with true amplitudes (offset dependend scaling for geometric spreading is applied for the wiggle traces. The colored backgroud image is scaled with AGC equivalent to the

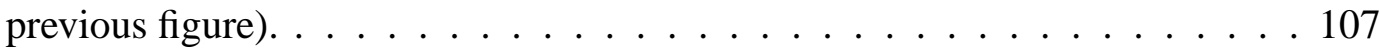

7.6 The spectral coefficients in the frequency-slowness domain have been obtained from successive $\tau-p$ and Fourier transforms of the seismic gather. The reflected and refracted energy is seen at $0.3 \mathrm{~s} / \mathrm{km}$ and $10 \mathrm{~Hz}$ while the three modes of the guided wave tend to the slowness of $0.7 \mathrm{~s} / \mathrm{km}$ corresponding to the acoustic velocity in the water. . . . . . . . . . . . . . 108

7.7 The dispersive seismic wavefield has regional variations in the two shot examples from seismic lines 5,20 and 21 which is expressed in the variation in position and amplitudes of the acoustic modes. . . . . . . . . . . . . 109

7.8 The reference model reflects the setting of a sub-marine permafrost overlain by a thawed layer fo sediments. The corresponding synthetic spectrum shows the acoustic guided modes in the slowness range corresponding to the limits from the water velocity and the permafrost compressional velocity. . . . . . 110

7.9 Snapshot of the shear (top) and compressional (bottom) wave energy of the synthetic seismic wavefield at 0.8 seconds after exciting an explosive source at zero offset computed with the FD-algorithm (see text for desctiption of arrivals) . . . . . . . . . . . . . . . . . . 11

7.10 The dispersion curves obtained from the normal mode solution for the reference model shows that the number of normal modes is much higher than the observed modes. . . . . . . . . . . . . . . . . . . . 11

7.11 Depth variation of the eigenfunctions (top) of horizontal and vertical diplacement and the corresponding sensitivity kernels with respect to shear wave veolcity indicate the penetration depth as a function of frequency. . . . . . . . 114

7.12 The comparison of the energy variation with depth for the fundamental (red) and first higher (blue) mode shows the deeper penetration for the higher mode at high frequencies. . . . . . . . . . . . . . . 111

7.13 From the partial derivatives of the misfit function with respect to the model parameter of the final inversion model the sensitivity of individual modes to a certain model parameter can be investigated. The sensitivity to variations in Vp and Vs (top) and the density (bottom) of the sediment layer as well as Vs in the permafrost (bottom right) are plotted. Note the different absolute amplitudes of the scale $\left(\mathrm{A}(\alpha)=0.55, \mathrm{~A}(\beta)=2.4, \mathrm{~A}(\rho)=0.29, \mathrm{~A}\left(\beta_{\text {perm }}\right)=0.24\right) . \quad 116$ 
7.14 The presence of a thick permafrost layer $(>100 \mathrm{~m})$ gives rise to additional modes below $250 \mathrm{~s} / \mathrm{km}$ with increasing number for a thicker layer. The leaky mode energy for the $30 \mathrm{~m}$ layer (top right) is in smooth transition to the normal modes. For the other images a more sudden change in amplitudes is observed at the slowness corresponding to the p-wave velocity of the permafrost layer. . . . . . . . . . . . . . . . . . . . 117

7.15 The variation of shear wave velocity affects the dispersion pattern most for low shear wave velocites. The amplitudes of the acoustic modes are restricted to small patches. This becomes less severe as the Poisson number tends to $1 / 4$, i.e. the sediment corresponds to the normal compressional to shear wave velocity ratio of $\sqrt{3}$ commonly used for consollidated sediments and rocks.

7.16 The variation of the sediment layer thickness influences the amplitude distribution of the acoustic modes significantly. A thick sediment layer causes interference patterns and discontinous acoustic modes while a thin layer bounds most energy to the permafrost layer velocity. The $5-10 \mathrm{~m}$ thick layer has the smoothest and highest amplitudes of acoustic guided modes. . . . . . . . . 120

7.17 The comparison of the intermediate and final models (right) indicates the variability of the results if only limited a priori knowledge is used. The water depth changed significantly from $44 \mathrm{~m}$ to $34 \mathrm{~m}$. . . . . . . . . . . . . . . 122

7.18 The wavefield spectrum of the intermediate model (top) obtained from the travel time inversion does not fit the measured wavefield spectrum indicated by the solid lines. The synthetic wavefield spectrum of the final model (bottom) gives an acceptable fit. . . . . . . . . . . . . . . . 123

7.19 Resolution analysis for the final inversion model. . . . . . . . . . . . . . 124

7.20 The resolution analysis of the shear wave velocity in the permafrost compared with the density of the sediment layer indicate the trade-off between these two parameters. Remarkably is that the trade-off for variations in Vs to the sediment density is stronger than vice versa. . . . . . . . . . . . . 125

8.1 Location map of selected seismic lines and gravity coring and OBS positions in the two study areas in the Arkona Basin. Also marked is the location of the OBS station in the Tromper Wiek and the 'Petrobaltic' well G14. . . . . . 129

8.2 The towing-depth of the streamer is determined from the forces acting on the system as indicated by the arrows (a). The observed shape of the towed streamer (b) could possibly be improved by the application of a depressor weight (c) . . . . . . . . . . . . . . . . . . . . 133

8.3 Depth control of the deep-towed streamer was monitored with a) the depth of the four streamer bird and $b$ ) the fin angle for each of the birds. . . . . . . 134 
8.4 Arrival times of the direct wave and ghost arrivals for the deep-tow streamer configuration. . . . . . . . . . . . . . . 136

8.5 a) Deep-tow streamer gather and b) single-channel section of channel 33 (220 $\mathrm{m}$ offset)

8.6 Seismic lines of the deep-tow experiments in the south-eastern rim study area. Airguns of 2.51 and 1.21 were used on profiles P15-17 and P24,25, respectively. The small airgun (0.1 1) was used for surface towed streamer profiles P27-28. The location of OBS 54 is marked by the circle. The locations where interface wave energy was observed in the streamer sections is marked by grey patches along the seismic lines. The numbered crosses indicate the shot positions of the data examples from the deep-tow streamer experiments. . 138

8.7 High resolution seismic section coming into the central basin area. . . . . . . 140

8.8 Line drawings of high resolution seismic data sets in study area I. . . . . . . 141

8.9 The sub bottom profiler section from study area II shows the structure of the dominant layers. . . . . . . . . . . . . . . . . . . . . 142

8.10 Line drawings of high resolution seismic data sets in study area II. . . . . . . 143

8.11 Refraction analysis of shot gather from data set II.2 . . . . . . . . . . . . . 143

8.12 Start models for the two sites derived from the refraction analysis and interpretation of the seismostratigrahpic sequences. . . . . . . . . . . . . . 144

8.13 Common-Receiver-Gather of A1201-P47 recorded on OBS 45 in the Central Arkona Basin. . . . . . . . . . . . . . . . . . . . 145

8.14 Time reduced zoom of the CRG in fig. 8.13. . . . . . . . . . . . . . 146

8.15 Low pass filtered section of A1201-P47 recorded at OBS 45. The wiggle traces are scaled depending on offset while the color image was AGC amplified. No Scholte wave energy is observed below $20 \mathrm{~Hz}$. . . . . . . . . . . . 147

8.16 Common-receiver-gather of seismic line Al201-P59 recorded at OBS 54 in the South-Eastern rim study area. The dispersive interface wave visible in both branches of the split-spread recording has phase velocities of 400-800 $\mathrm{m} / \mathrm{s}$ and a group velocity of approximately $250 \mathrm{~m} / \mathrm{s}$. . . . . . . . . . . . 148

8.17 The interface wave of A1201-P59 (Fig. 8.16) has been enhanced by low-pass filtering and the application of time domain tapers. This is the input data set for the modified Fourier-Bessel transform. . . . . . . . . . . . . . . . . . . . 149

8.18 Slowness spectra of the deep-tow streamer shot 313 on seismic line Al188P15 (left) and of line Al201-P59 recorded at OBS 54 in the south-eastern rim study area. . . . . . . . . . . . . . . . . . . . 150 
8.19 Slowness spectra of Al201-P59 recorded at the hydrophone component of OBS 54 from the south-eastern rim of the Arkona Basin (left) compared with the vertical component recording of a planted seismometer in the Tromper Wiek from Al201-P23 . . . . . . . . . . . . . . . . . . . 151

8.20 The wave spectra for two shots of seismic line Al188-P16 about $2 \mathrm{~km}$ apart indicates lateral variations of the dispersive mode which can be resolved with the towed acquisition system.

8.21 The slowness spectrum for the seismic line Al201-P47 (Fig. 8.13 and 8.14) was recorded in the central Arkona Basin on OBS 45. The presence of 6 higher modes within the frequency range from 20 to $120 \mathrm{~Hz}$ corresponds to the guiding influence of the water column. Note the variation of amplitudes of the individual modes as well as the "feathering" below slowness values 0.4 $\mathrm{s} / \mathrm{km}$ for the second and third higher modes. . . . . . . . . . . . 153

8.22 Final models of the inversion result . . . . . . . . . . . . . . 154

8.23 Final model and inversion result. . . . . . . . . . . . . . . . . 155

8.24 Synthetic slowness spectrum of the final model with dispersion curve picks from the measured data. . . . . . . . . . . . . . . . . . . . 156

8.25 Resolution analysis results for all model velocities and layer thicknesses. . . . 157

A.1 Offset variation in synthetic seismograms lead to an simulated error of the OBS location from the navigation data. . . . . . . . . . . . . 187

A.2 Reducing the offset range used for the wavefield transform at the near-field side demonstrates that the simulated navigation errors are not relevant for measurements at distances of about the double of the navigational error. . . . 188

B.1 Influence of temperature and salinity on sea water sound speed profiles . . . . 190

B.2 Depth control system on day one of the deep-tow testing phase . . . . . . . 192

B.3 Single channel sections of channel 33 at $220 \mathrm{~m}$ offset from the 2.51 airgun source. . . . . . . . . . . . . . . . . . . . 193

B.4 Single channel sections of channel 33 at $220 \mathrm{~m}$ offset. . . . . . . . . . . . . 194

B.5 Shots 19387 and 19397 of line Al188-P16. For practical reasons the first two digits of the shot numbers were ommitted in the axis labels of the previous figures. . . . . . . . . . . . . . . . . . 195 


\section{List of Tables}

2.1 Initial and boundary conditions . . . . . . . . . . . . . . 22

2.2 Interface wave types as classified by Rauch (1980). The subscript $l$ denotes the fluid properties of the liquid halfspace and s the solid properties, subscripts $s 1$ and $s 2$ denote the solid upper and lower halfspaces respectively.

5.1 Control parameters for the inversion of full wave spectra. The error levels for the travel times and greens coefficients are denoted $t t_{e r r}$ and $g_{e r r}$ and correspondingly the misfit functions $\chi_{t t}^{2}$ and $\chi_{g r e e n s}^{2}$ for the data sub sets of the travel time and greens coefficients. . . . . . . . . . . . . . 68

6.1 Comparison of the sensor systems $\ldots \ldots \ldots$. . . . . . . . . . 90

8.1 Stratigraphic sequences of the Baltic Sea classified according to Endler (1989) and Lemke (1998). . . . . . . . . . . . . . . . . . . . . . . . 139

9.1 Observed seismic wave types presented in the case studies in part II. . . . . . 161

9.2 Acquisition parameters required (used) for different sediment and wave types (interface; guided). The variation of acquisition parameters is much higher for the interface waves than for guided waves. The required shot/receiver spacing render Scholte (interface) wave measurements in soft clay or mud unfeasible. 


\section{Appendix A}

\section{Methods}

\section{A.1 Acquisition Restrictions of the Stationary-Receiver Ex- periment}

The derivation of the relation between the lowest resolvable shear wave velocity and the offset, ship speed and lowest determinable frequency in an walk-away seismic experiment us founded on the sampling theorem

$$
c_{\text {min }}=4 \pi \Delta x f_{\text {min }} \Rightarrow \Delta x=\frac{c_{\text {min }}}{4 \pi f_{\text {min }}}
$$

The following relations are used to express this relation of the lowest resolvable shear wave velocity $c_{\min }$ in terms of the maximum distance from the receiver $X_{\max }$, the profiling speed of the research vessel towing the seismic source $V_{\text {ship }}$ and the frequency of the interface wave to be measured $f_{\min }$. The shot spacing is determined by the minimum recording length, i.e. the time of energy propagation of the Scholte wave to arrive at the maximum distance of observation.

$$
\begin{array}{ccc}
V_{\text {ship }} T_{R}=\Delta x & \Rightarrow \quad T_{R}=\frac{\Delta x}{V_{\text {ship }}}=\frac{c_{\text {min }}}{4 \pi f_{\min } V_{\text {ship }}} \\
V_{\text {min }} T_{R}=X_{\text {max }} & \Rightarrow \quad V_{\text {min }} c_{\text {min }}=4 \pi f_{\text {min }} X_{\text {max }} V_{\text {ship }}
\end{array}
$$

With an approximation of the Scholte wave velocity to be less than 0.8 times the lowest shear wave velocity to be resolved this leads to the relation presented in the text.

$$
V_{\min } \geq 0.8 c_{\min } \quad \Rightarrow \quad c_{\min }^{2}=5 \pi f_{\min } X_{\max } V_{\text {ship }}
$$




\section{A.2 Influence of Acquisition Geometry}

The Influence of the acquisition geometry for a stationary-receiver experiment was investigated with a synthetic seismic wavefield. First the wavefield transform in form of a frequencydomain slant-stack was performed with the synthetic seismogram with the acuisition geometry according to the model. Then the OBS position was changed sucessivly at 5-10 m intervals in cross-line direction. The source-receiver geometry was caclulated for this modified configuration, hence the seismiograms are calculated for a different position than the analysis performed. The error in terms of the offset corresponds to an absolute error in the OBS positioning for a real measurement, but is in the opposite direction. The sequence of wavefield spectra obtained for variations of the cross-line distance are plotted in the range from 10 $100 \mathrm{~m}$ (Fig. A.1). The subsequent elimination of near-field traces of the section to obtain a limited offset spread of the far traces only can compensate for the systematical navigation errors from above (Fig. A.2). 

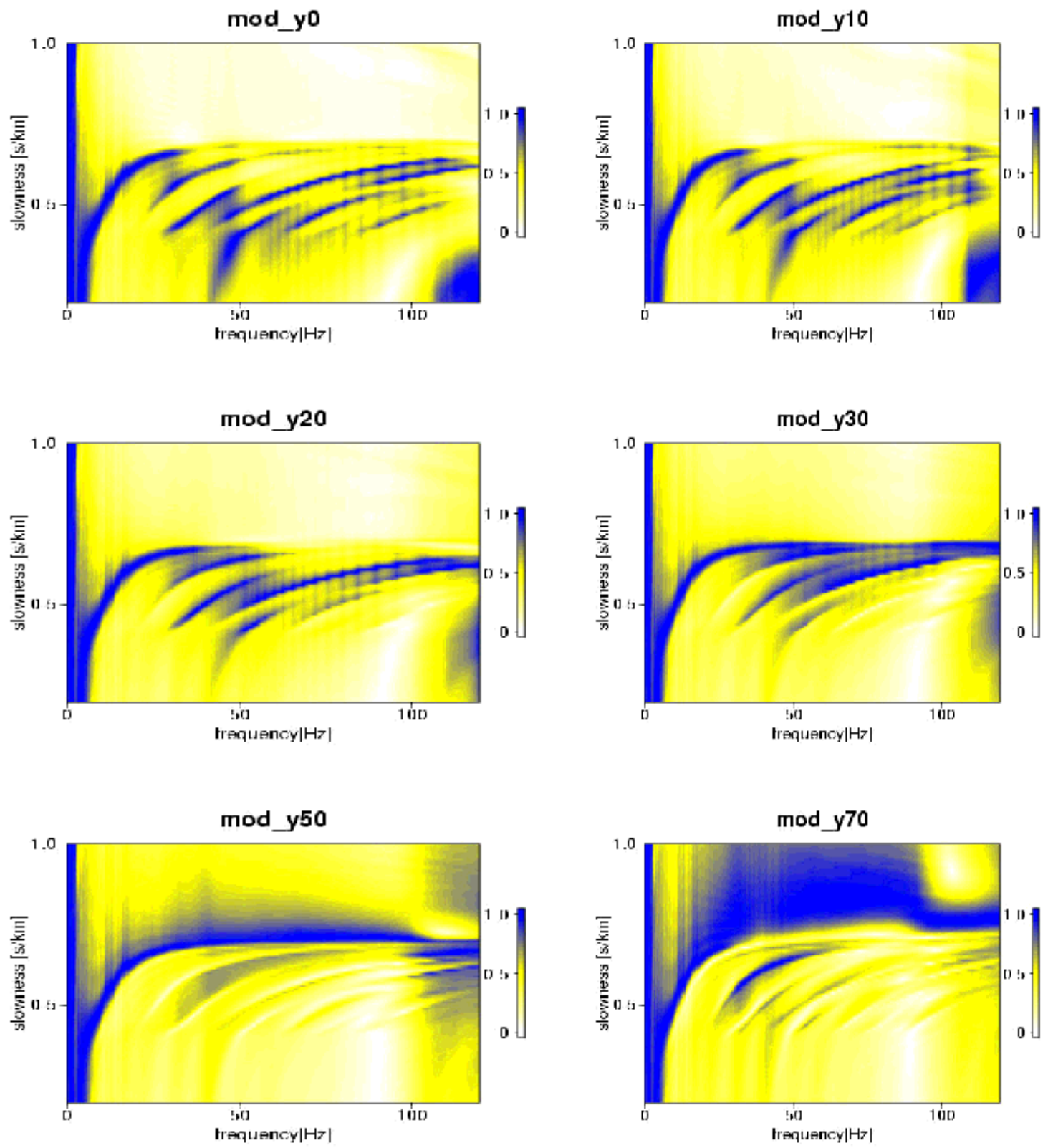

Figure A.1: Offset variation in synthetic seismograms lead to an simulated error of the OBS location from the navigation data. 

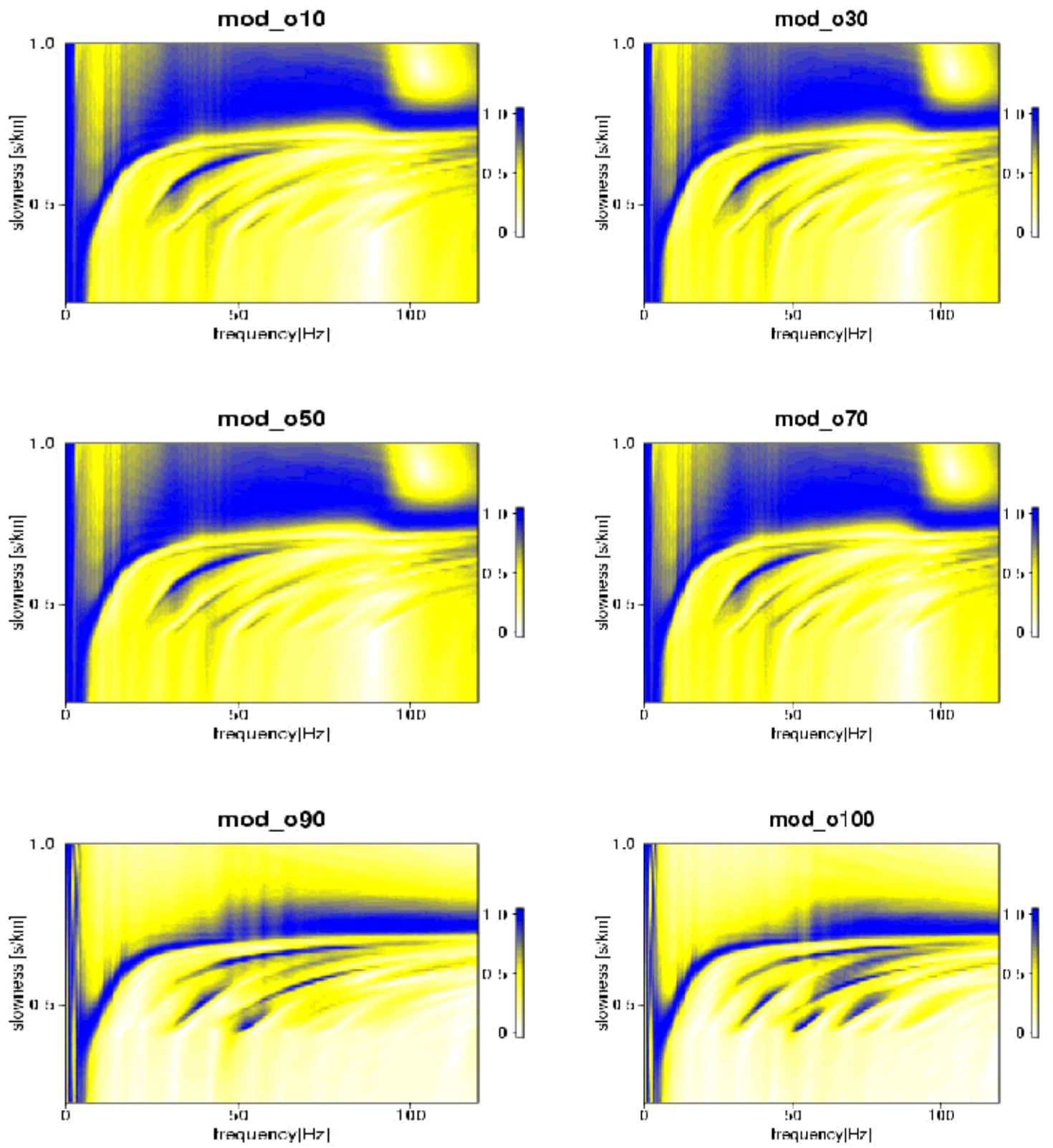

Figure A.2: Reducing the offset range used for the wavefield transform at the near-field side demonstrates that the simulated navigation errors are not relevant for measurements at distances of about the double of the navigational error. 


\section{Appendix B}

\section{Data Acquisition}

\section{B.1 Research Cruises}

\section{B.1.1 List of research cruises}

\begin{tabular}{l|l|l|l|l} 
Cruise & Area & Dates & No & Research Vessel \\
\hline Li199 & Kiel B. & 27.09. - 30.09.1999 & & FK Littorina \& FB Polarfuchs \\
Al160 & Arkona & 17.05. - 22.05.2000 & 160 & FS Alkor \\
Li200 & Kiel B. & 28.08. - 31.08.2000 & & FK Littorina \& FB Polarfuchs \\
Po266 & Arkona & 08.01. - 12.01.2001 & 266 & FS Poseidon \\
Al174 & Kiel B. & 05.02. - 09.02.2001 & 174 & FS Alkor \\
Al181 & Ark/K.B. & $14.05 .-18.05 .2001$ & 181 & FS Alkor \\
Al188 & Arkona & $18.08 .-24.08 .2001$ & 188 & FS Alkor \\
Al196 & Arkona & $11.02 .-15.02 .2002$ & 196 & FS Alkor \\
A1201 & Arkona & $10.05 .-19.05 .2002$ & 201 & FS Alkor
\end{tabular}

B.1.2 List of stations and profiles of the cruises Po266, Al188 and Al201 


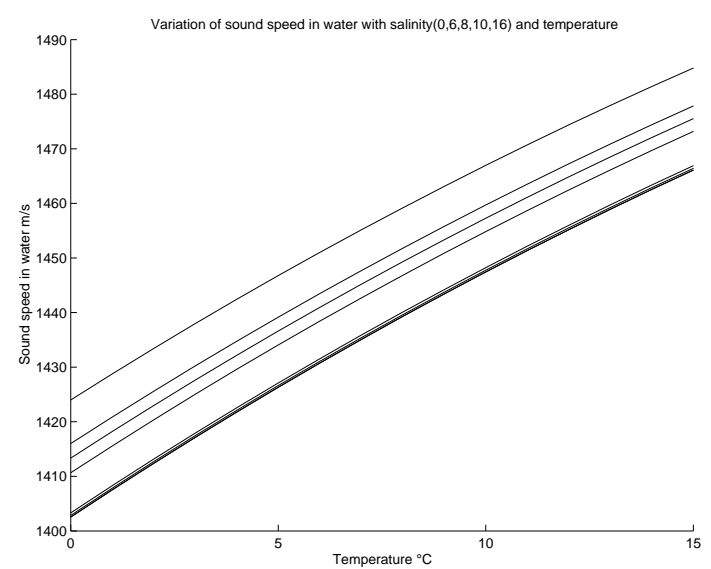

(a) variation of sound speed with salinity and temperature

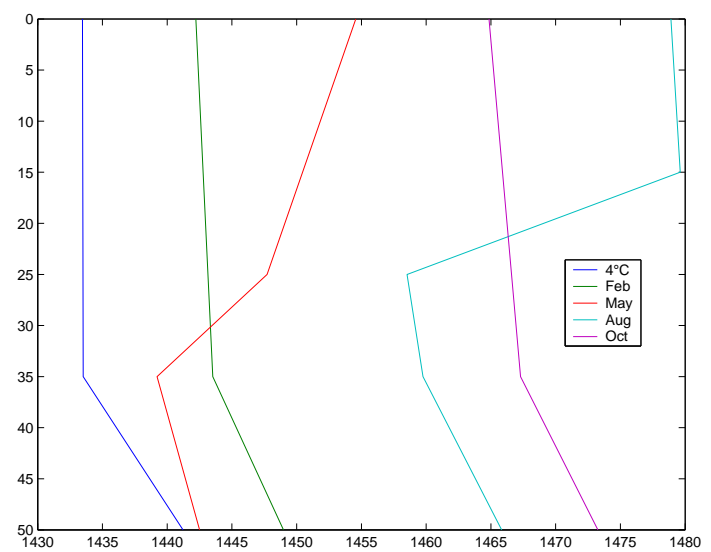

(b) depth profiles of sound spees in water for the temperatures and salinities reported sound speed profiles

Figure B.1: Influence of temperature and salinity on sea water sound speed profiles

\section{B.1.3 Corrections for Sound Speed in Water}

Since the absolute position of the OBS stations deployed on cruise Al201 was obtained by measuring the travel time of the direct p-wave, the sound speed of water had to be used. The indication of very low sound speeds in the data gave rise to cross check the plausibility of the inversion result in terms of physically sensible of sound speed in water for the Arkona Basin. Therefore the hydro-chemical setting of the Arkona Basin as described by Matthäus et. al. (Matthäus et al., 2001) was used to compute typical sound speed profiles for the water column using the procedures proposed by UNESCO (Anonymous, 1985).

The speed of sound in water is a mere function of the density, or in terms of observables a function of temperature, salinity and pressure. Both temperature and salinity variations within the year 2000 have been studied and reported by (Matthäus et al., 2001) and we produced sound speed profiles for February, May and End of July representing the cruises in winter (Po266,Al196), spring (Al160,Al181,Al201) and summer (Al188).

\section{B.1.4 Data conversion and preparation}

All seismic data was converted to the SEG-Y derived Seismic Unix data format. The MCS data was recorded in SEG-D Rev 8058 format, the SBP data in Microsoft-audio(.wav) format, and the OBS data either in PASSCAL or SEDIS format. All conversions were performed on 
a Linux PC using factory supplied or at IFG developed conversion software. Some conversion procedures to extract common receiver gathers from the OBS data had to be developed for the new OBS system (Appendix B.1.4). Manual preprocessing usually included editing, detrending with a constant average, offset dependent scaling, filtering and tapering and was performed with standard or slightly modified versions of the Seismic Unix package. (Source code of all modified modules is provided on the acompaning CD or the IFG website).

\section{B.1.5 Navigation}

The aim to link the navigation data with the seismic data is twofold. Dominantly it is important to reflect the acquisition geometry, i.e. the relative position between seismic source and receivers. Secondly the absolute position is required in order to link the information of the different profiles and also previous research work. This implies also, that the geometry setting requires high accuracy of the relative positions between source and receivers, while for interpretation of the data requirements on navigation accuracy are less. To accomplish the latter, correction for the distance between the GPS antenna and the actual source position have generally not been applied (e.g SBP data). However, the calculation of the survey geometry required to account for those corrections whenever the survey geometry was not fixed in relation to the shipboard GPS antenna, e.g for the OBS measurements.

Generally the navigation data from the shipboard differential GPS system has been used when available. Therefore the navigation data was linked to the seismic data by UTC time keeping. The Meinberg time server was installed within the local network to keep all recording units to UTC time during measurements when possible. The time keeping for the OBS data was achieved, by synchronising the clock of the recorder at the beginning and measuring the skew at the end of the recording.

OBS geometry For the geometry calculations for OBS configurations absolute position of the source as well as the OBS stations had to be calculated. The source position projected to the surface was calculated and the source and receiver depth treated in the further processing. The position of the OBS was recorded at surface deployment. Since the water depth was less than $30 \mathrm{~m}$ the drift of the OBS during sinking to the sea floor was neglected. The position of selected stations was checked by picking the travel times from at least 4 shot positions and inverting for the OBS location.

MCS geometry The geometry of the MCS-recordings have been calculated relative to the source position. The airgun source was placed either $25 \mathrm{~m}$ or $50 \mathrm{~m}$ from the stern of the vessel at various depth. While this was accounted for in calculating the source receiver geometry, the absolute position of the source was usually assumed to be at the antenna position. Also, the 


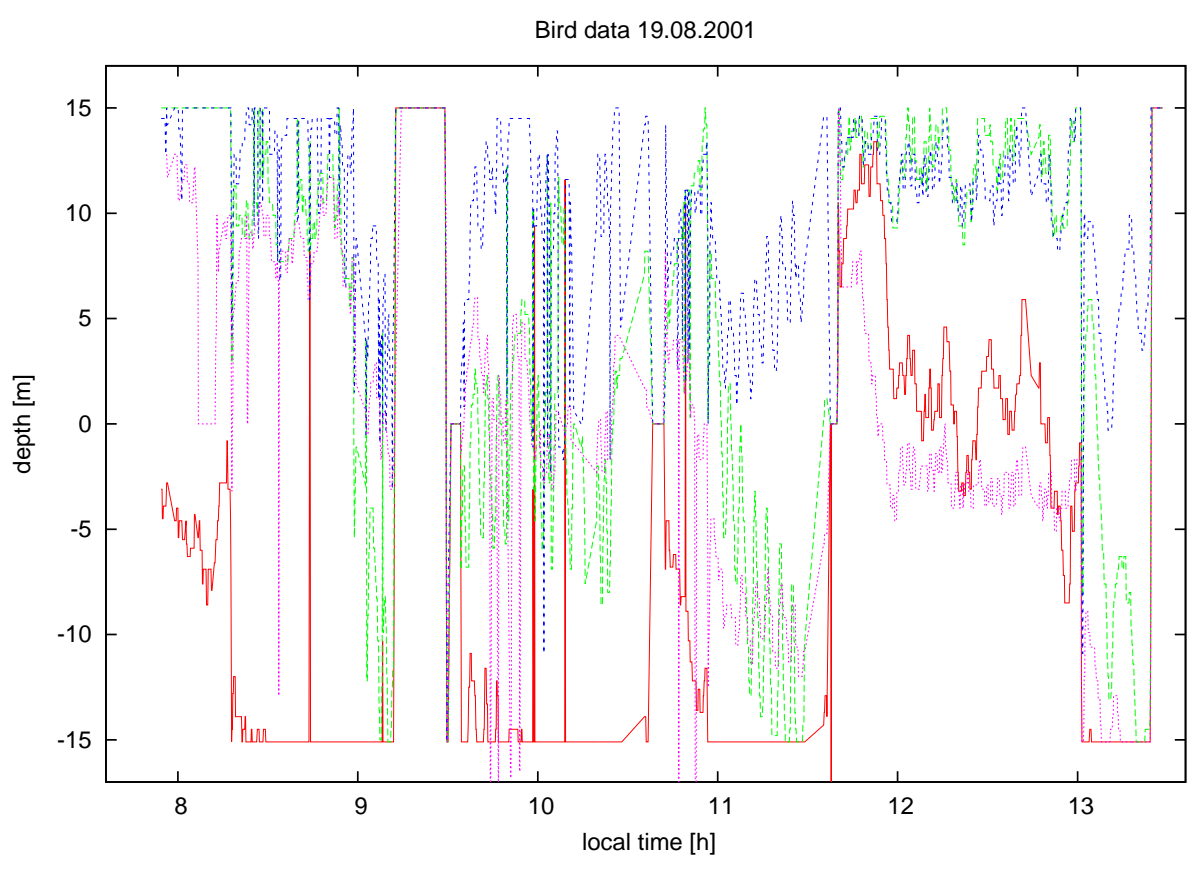

(a) fin angles (negative is downwards) of the individual birds.

Figure B.2: Depth control system on day one of the deep-tow testing phase

distance between source and first receiver of the streamer was assumed to be in direction of the profile only. Lateral distances of approximately $10 \mathrm{~m}$ have been neglected. The distance between the source and the first recording channel has been adapted according to changes in the acquisition, i.e. the length of the lead-in cable, towing depth of the streamer or the airgun etc.. All other recording channels of the streamer have been assumed to be at fixed spacing along the profile. Streamer feathering was not taken into account. More accurate geometry settings were tested with two profiles, but showed no significant improvement to the data. Therefore the crude geometry settings as mentioned above were assumed to be acceptable.

\section{B.2 Deep-tow streamer data}

\section{B.2.1 Deep-tow acquisition tests}

The depth control system of the streamer was used to monitor the streamer position as well as the applied finn angles of the streamer birds to maintain the streamer at this position. 


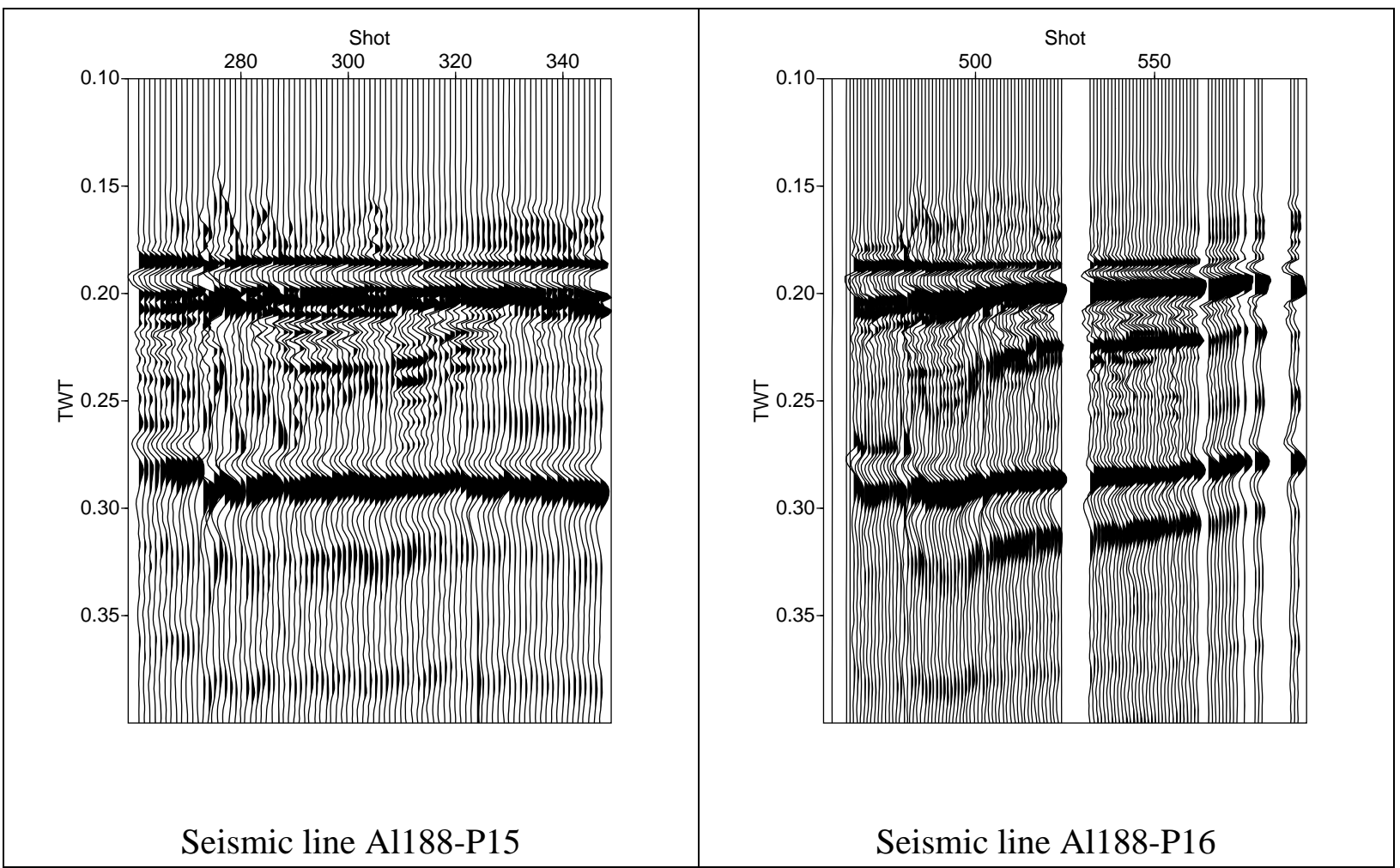

Figure B.3: Single channel sections of channel 33 at $220 \mathrm{~m}$ offset from the 2.51 airgun source.

\section{B.2.2 Single channel sections of deep-tow seismic lines}

In figures B.3 and B.4 the single-channel section of the seismic lines are presented where Scholte/Stonley interface waves have been observed.

\section{B.2.3 Selected shot-gathers of the deep-tow streamer lines}




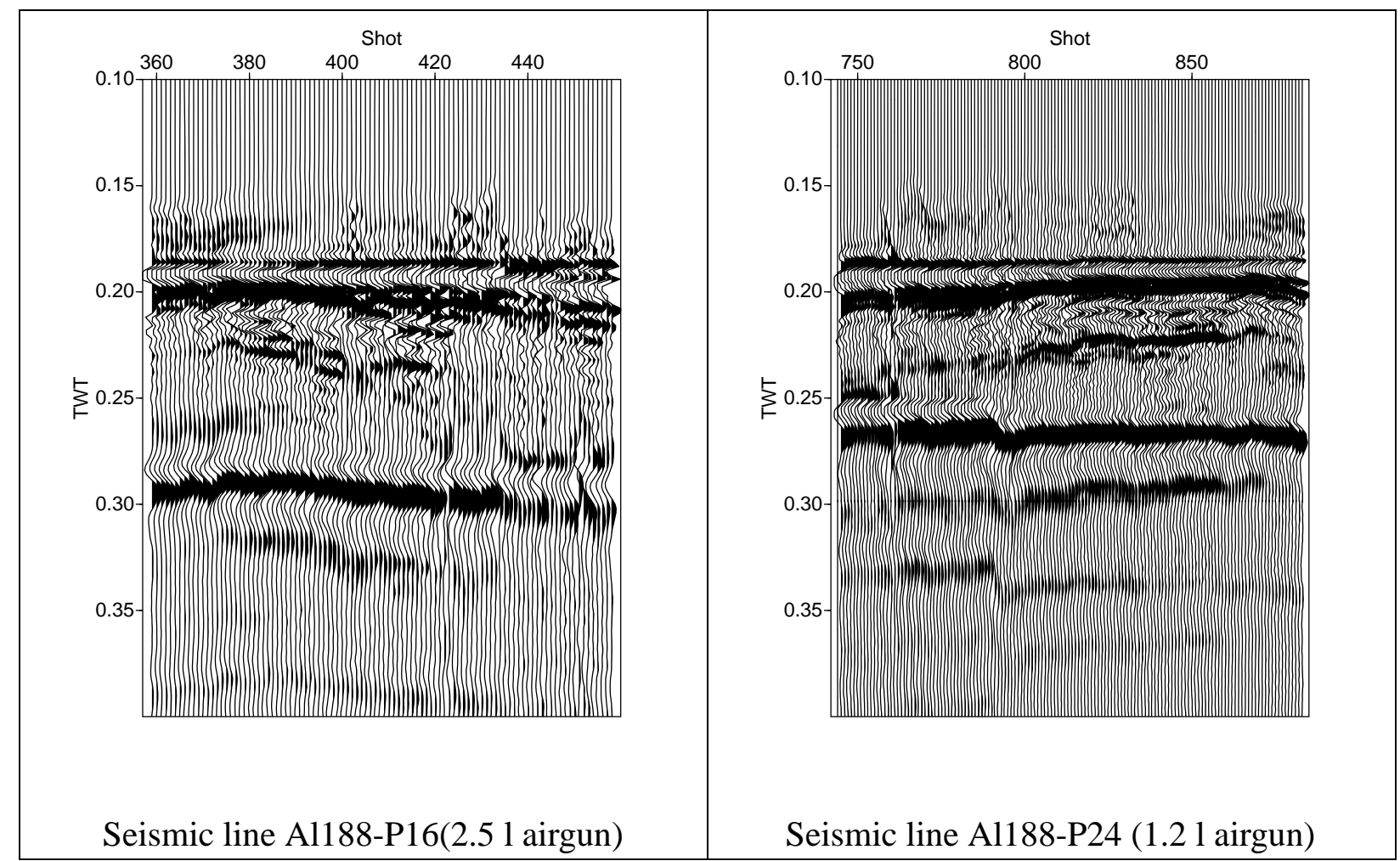

Figure B.4: Single channel sections of channel 33 at 220 m offset. 


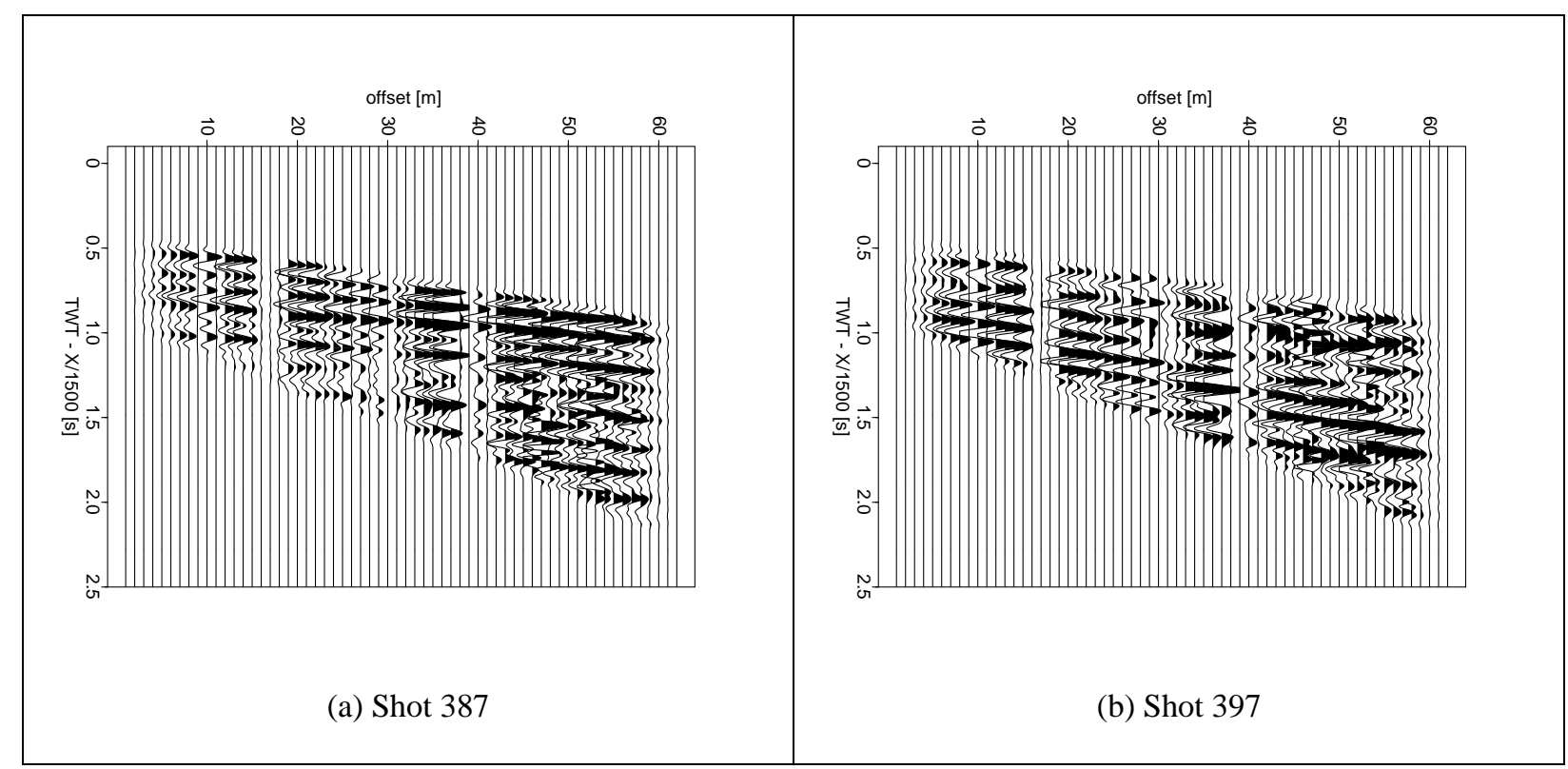

Figure B.5: Shots 19387 and 19397 of line A1188-P16. For practical reasons the first two digits of the shot numbers were ommitted in the axis labels of the previous figures. 


\section{Appendix C}

\section{Sensitivity of Inversion Parametes}

\section{C.1 Sesnsitivity of Acoutic Guided Wave Dispersion in Ma- rine Permafrost Conditions}

Parameter Variations

- Density

- Shear wave velocity

- Poisson ration

- Compressional velocity

- Layer thickness 
a) Sediment

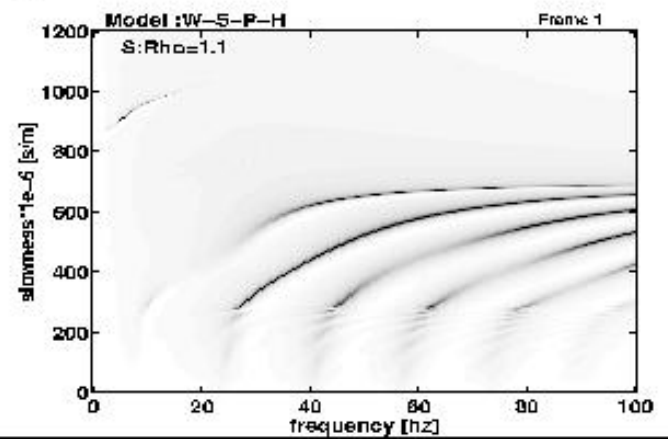

b) P'ermafrost
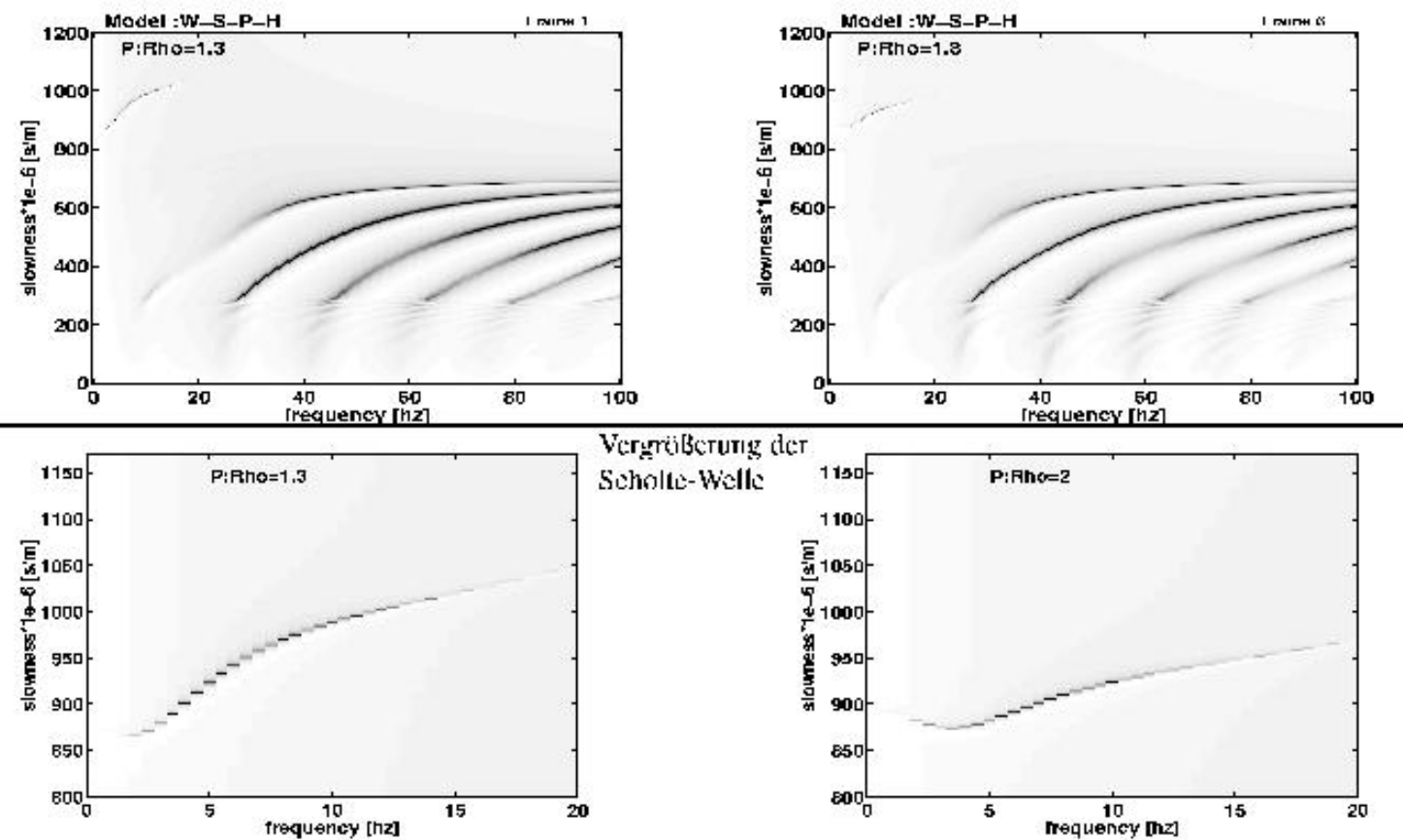

vereyribserutig der Soholtc-Willo

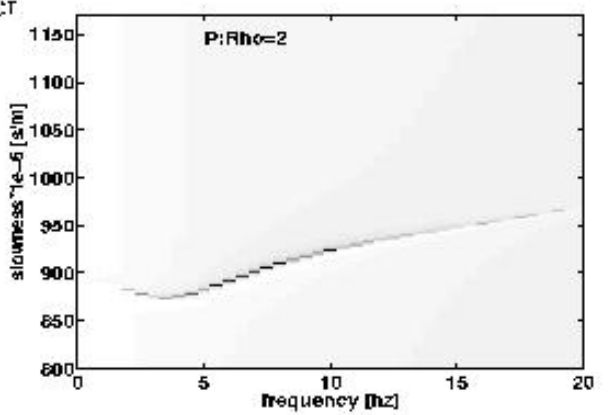

c) IIall)raum
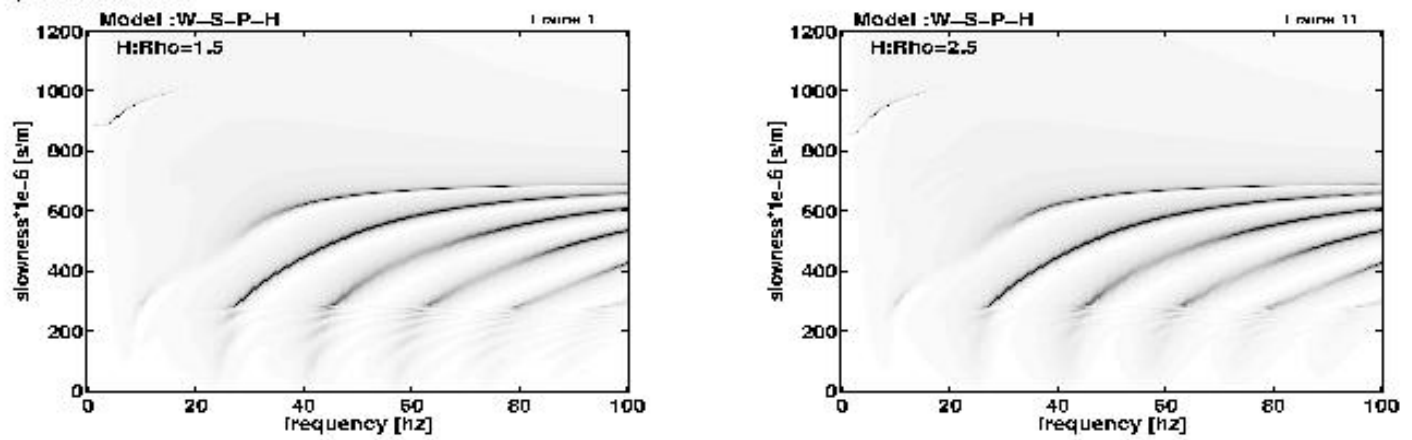


\section{Scherwellengeschwindigkeit}

a) im l'ermafrost
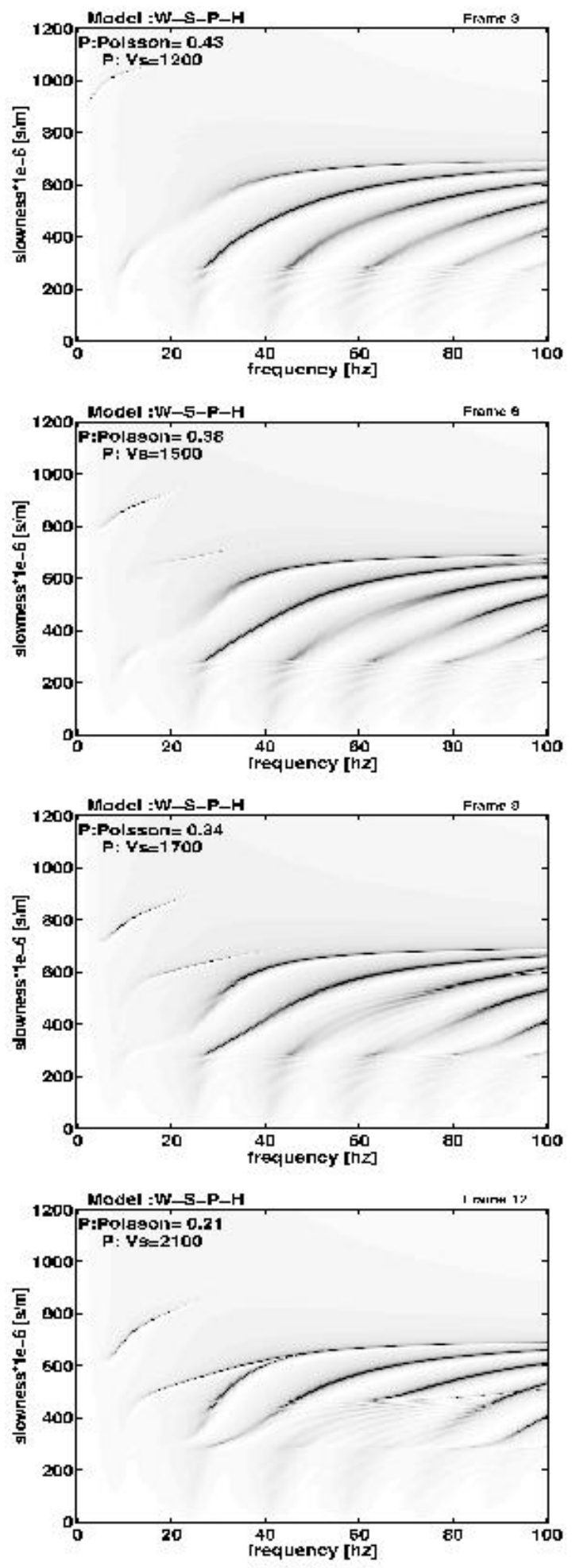

b) im Halloraum
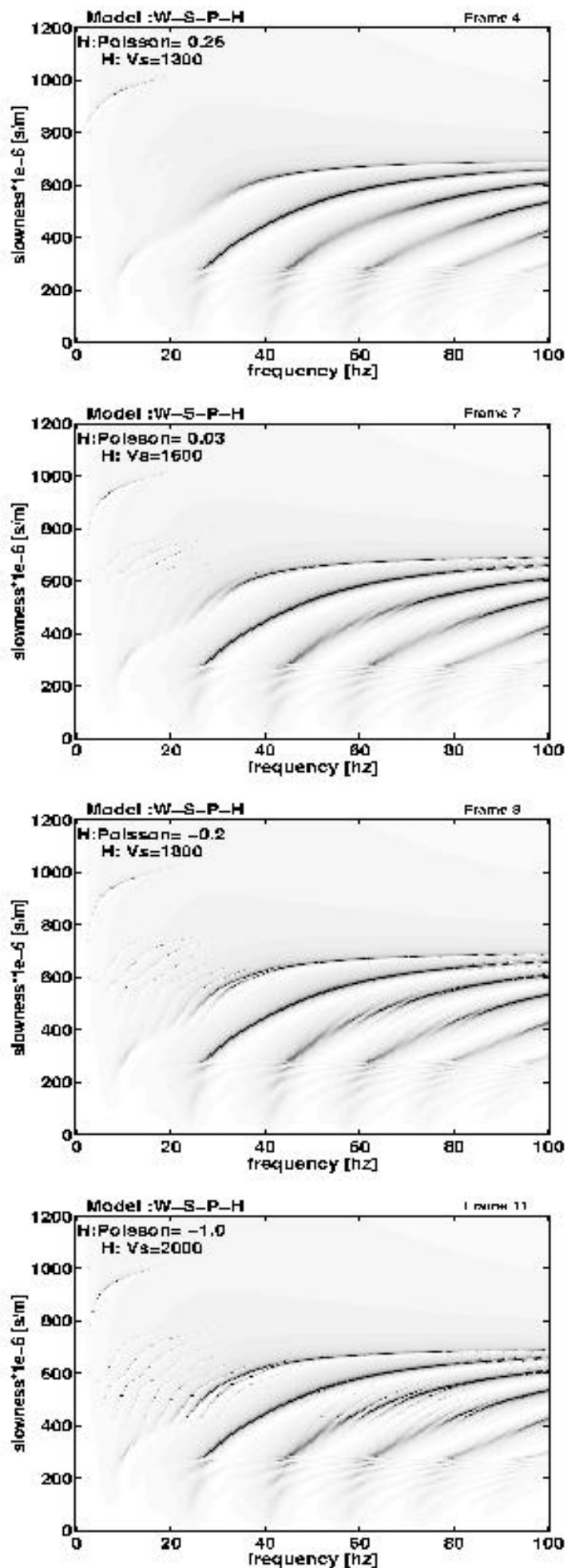
a) Scherwellengesch windigkeit
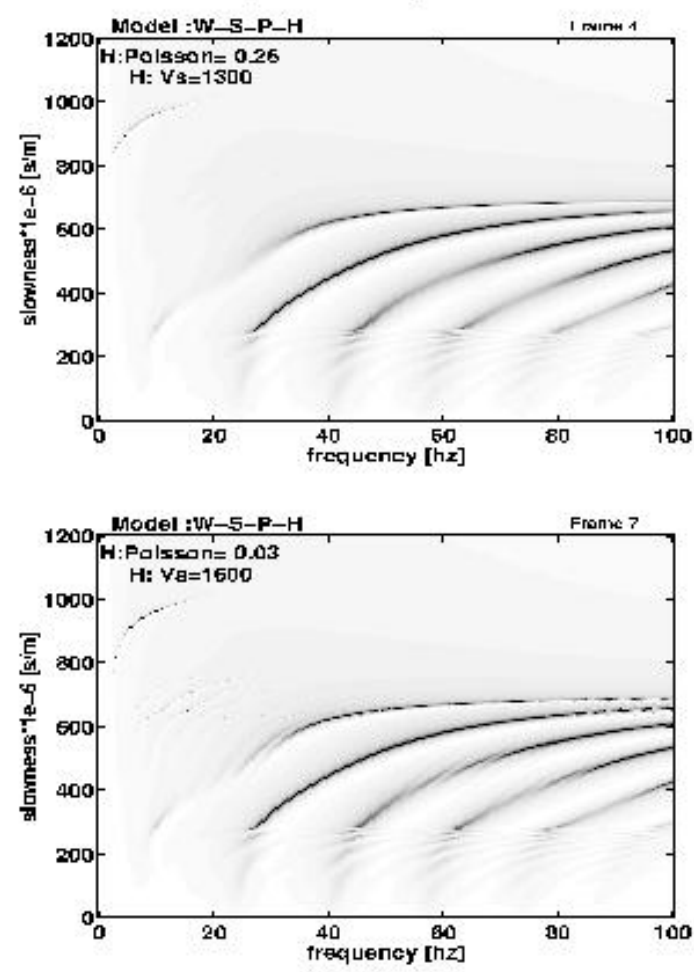

b) Kompressionswellengeschwindigkeit

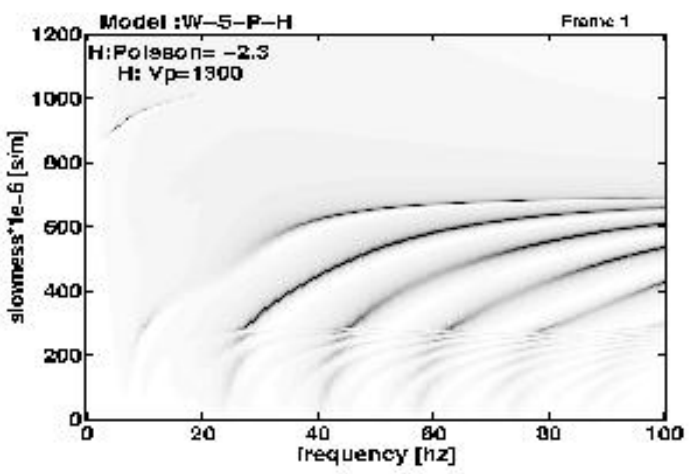

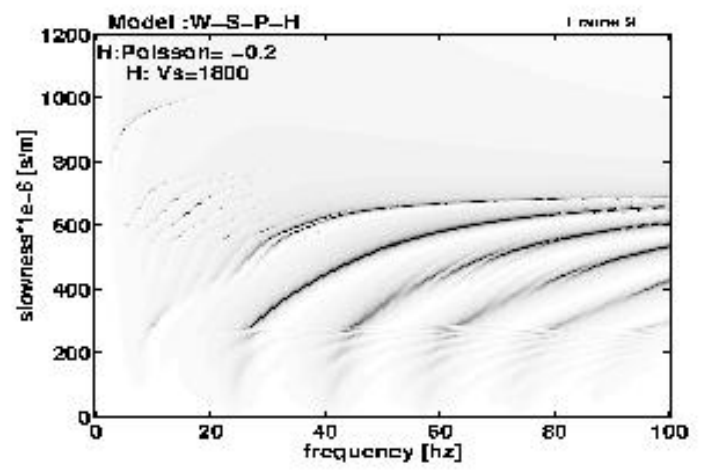
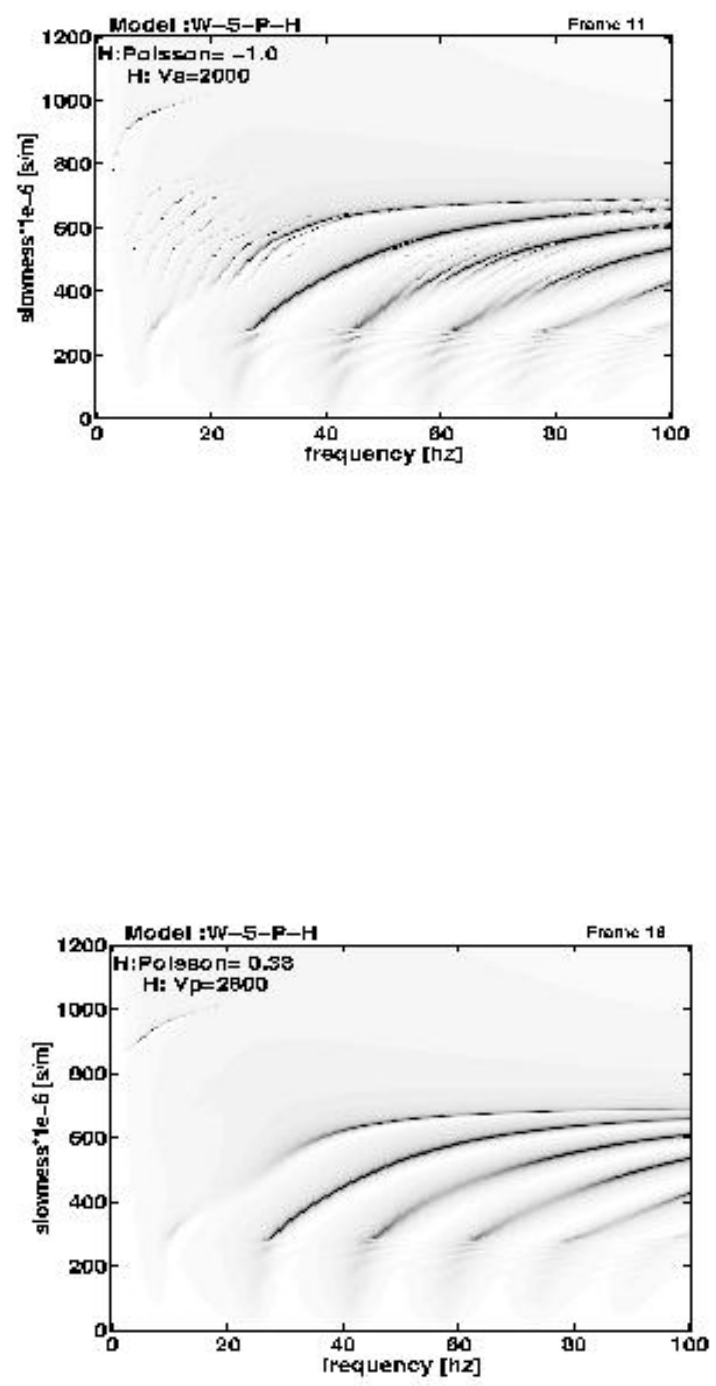

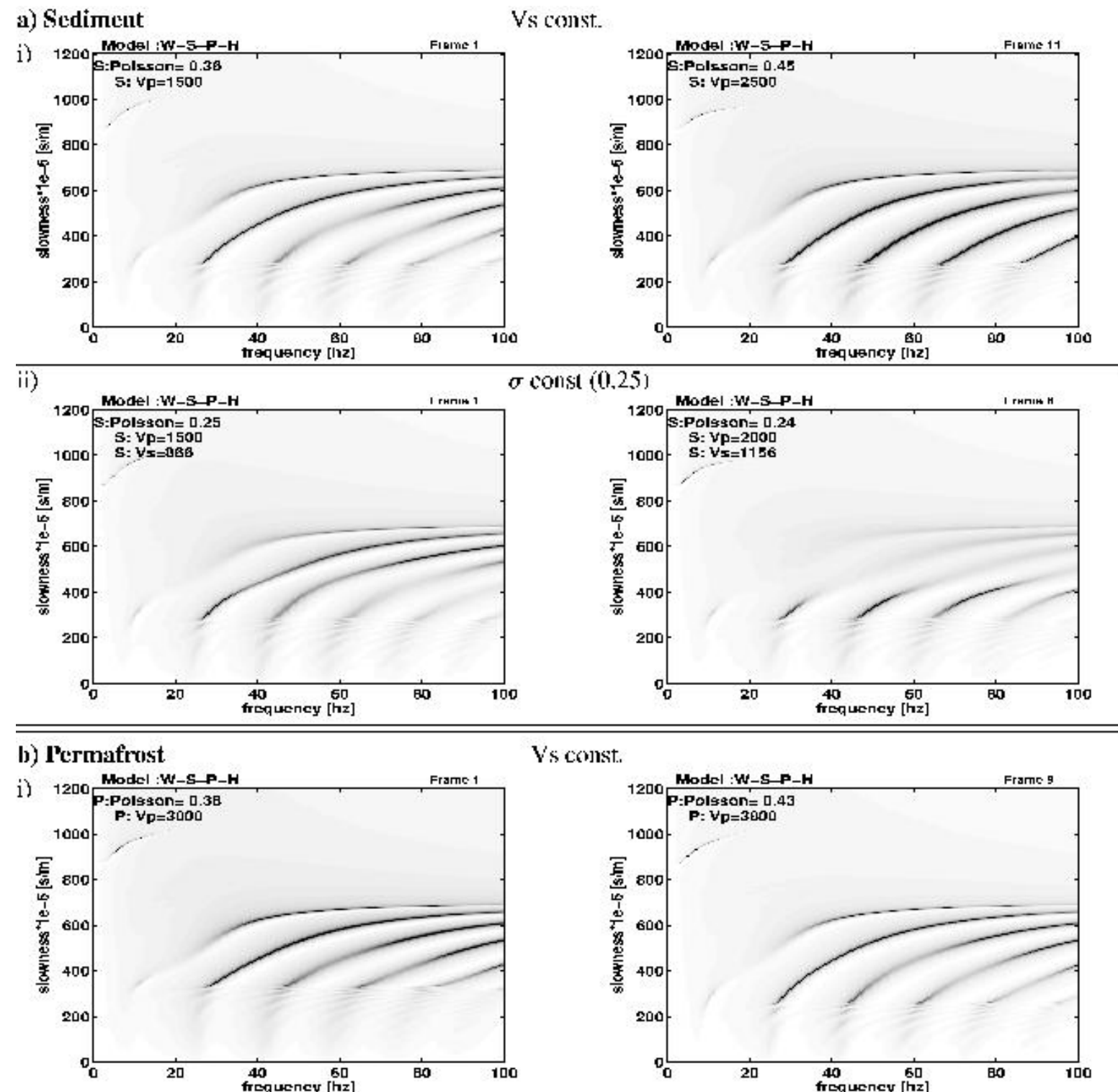

V's const.
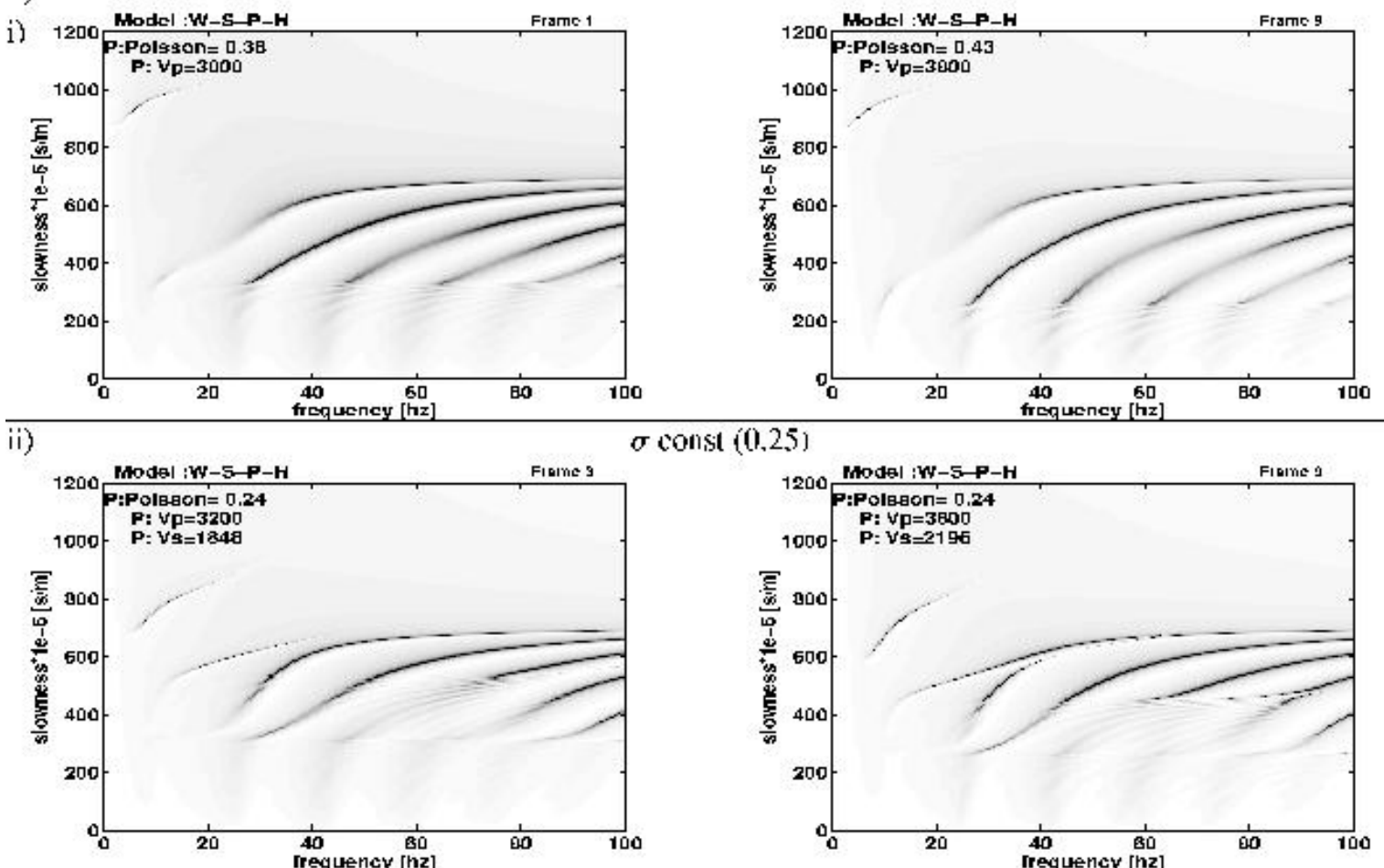
$\sigma$ const $(0,25)$

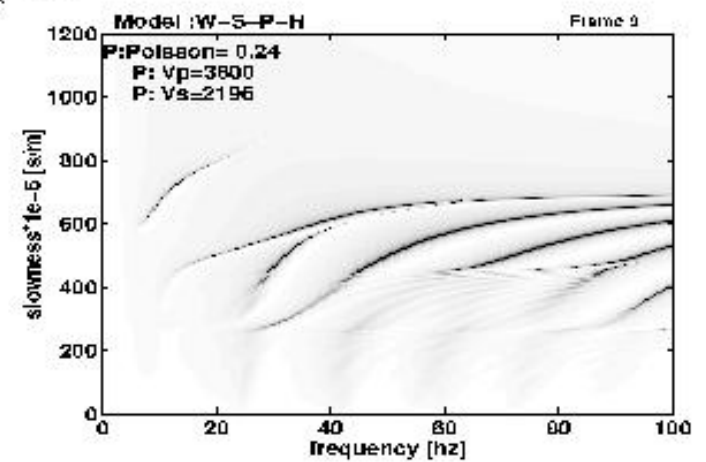


a) Wassertiele
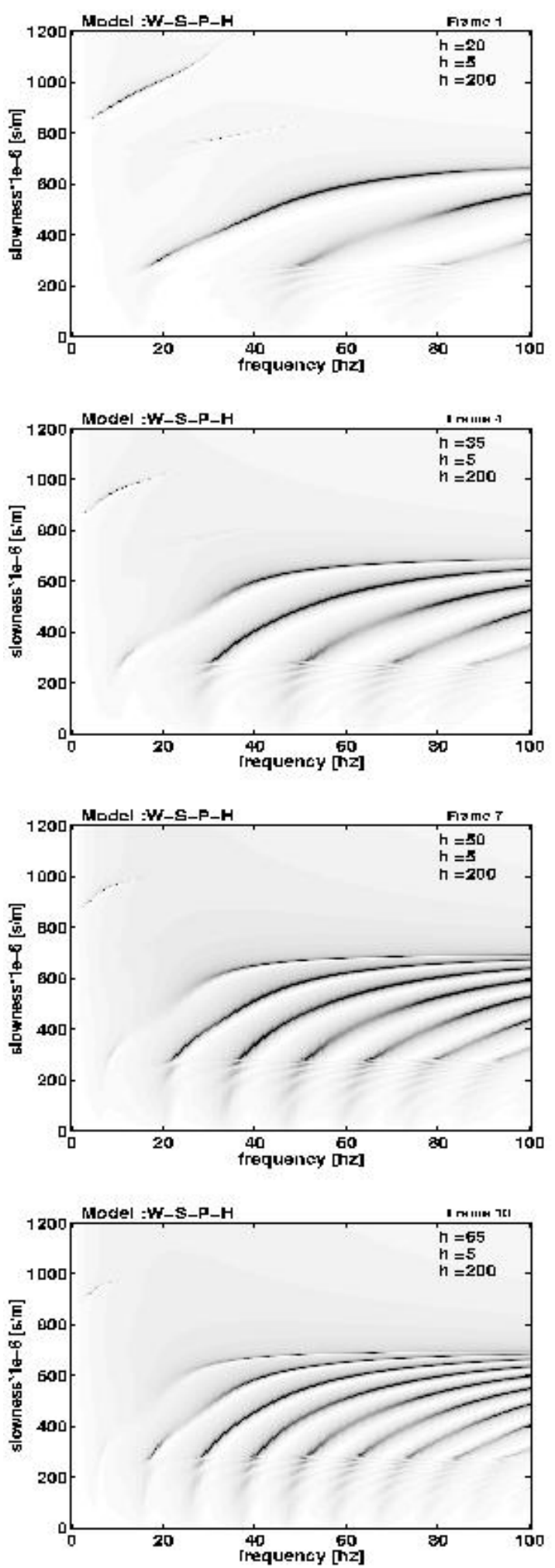

b) Sedimentächtigkeit
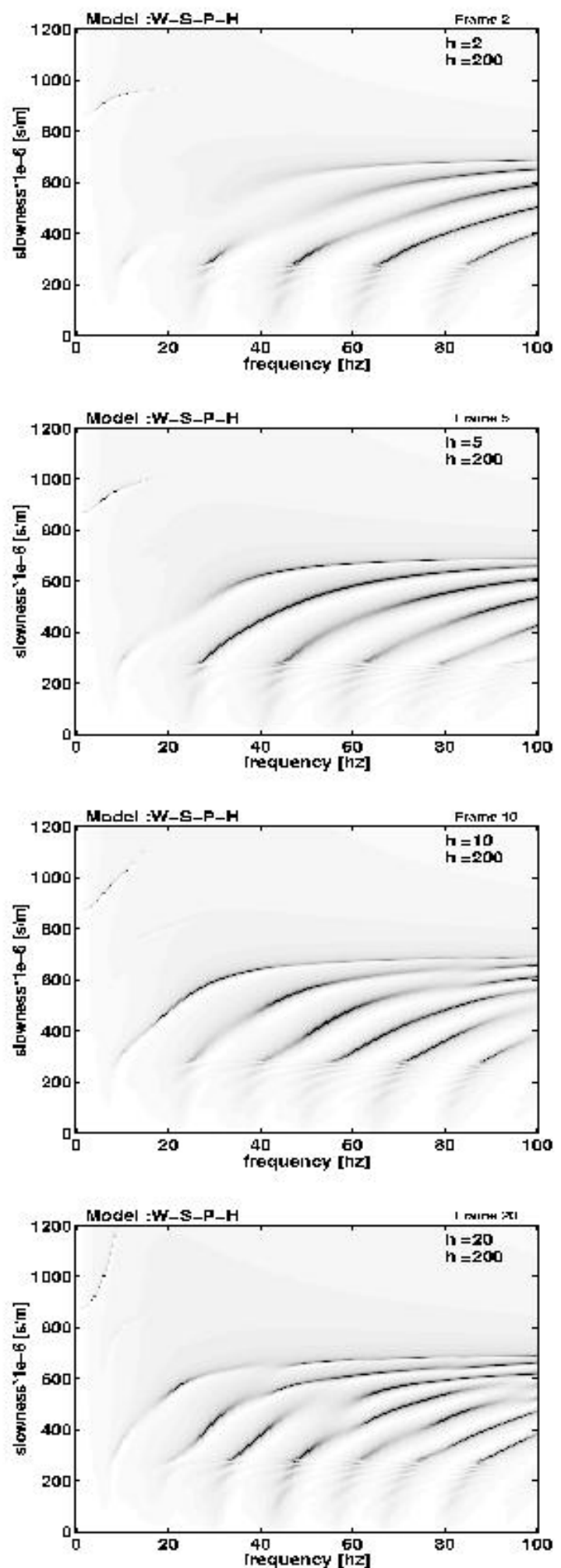
l'ermafrostmächtigkeit
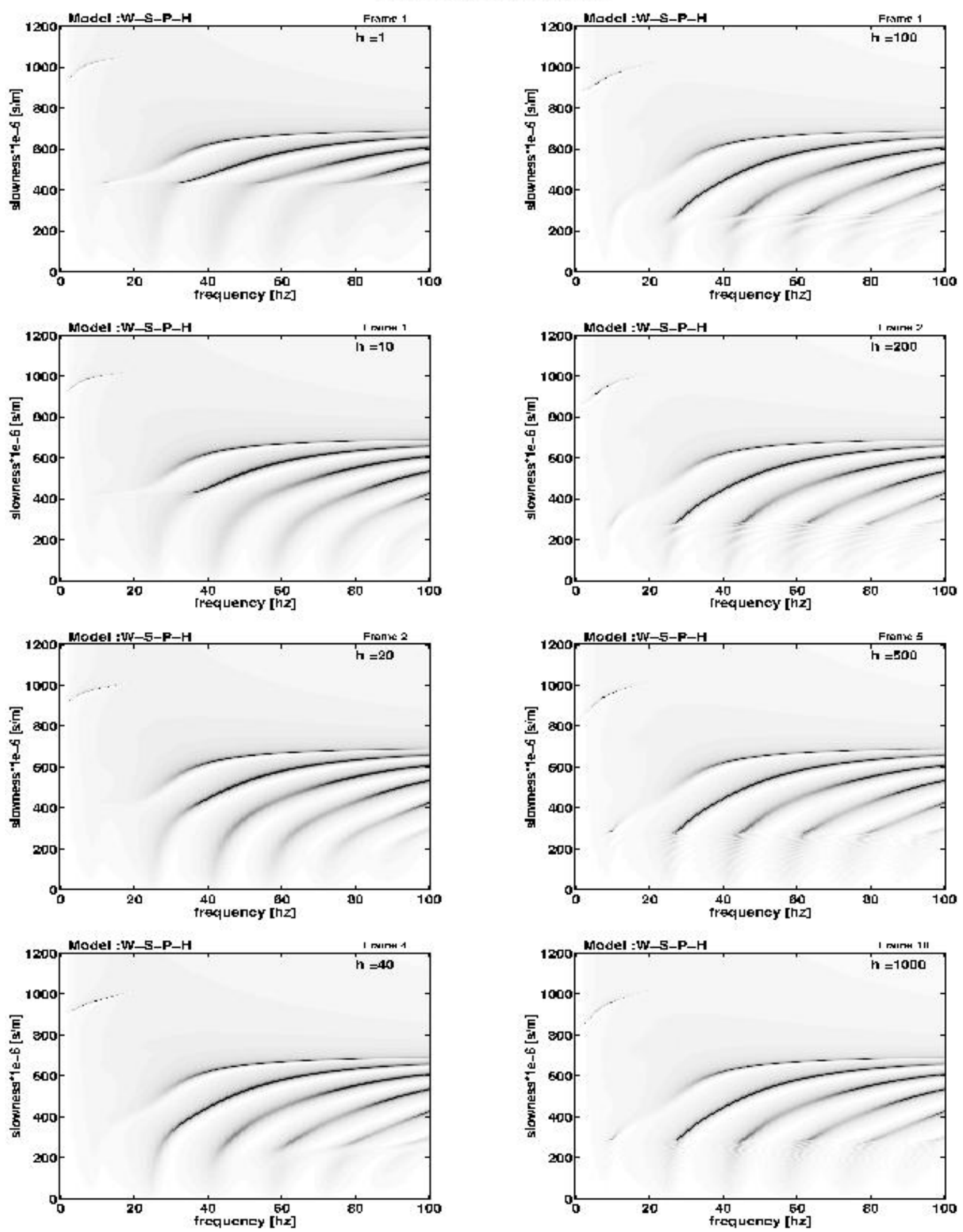


\section{Appendix D}

\section{Used Tools and Software}

The seismic equipment used for the various marine seismic experiments is mentioned with the data acquisition procedures. This is a list of other tools and computer software used which was either not mentioned thorughout the thesis or is here specified in more detail.

\section{D.1 Forward Algorithm}

\section{D.1.1 Propagator Matrix Algorithm MSEIS and MSEISFK}

The programs Mseis and MseisFK were provided by Wang, 1999 and 2001 in personal communication.

\section{D.1.2 Mode Summation Algorithm FLSPHER}

The normal mode summation program FLSPHER was provided by W. Friederich in personal communication. It is based on numerical itegration of the wave equation for a spherical earth model. Next to the dispersion relation the eigenfunction of the displacement as well as the sensitivity kernels have been used to study the variation of dispersion with model parameters.

\section{D.2 Seismic Processing}

\section{D.2.1 Standared Processing}

All standard seismic processing was performed with the Seismic Unix package from the Center or Wave Phenomena of the Colorado School of Mines (http://www.cwp.mines.edu/cwpcodes/) 


\section{D.2.2 Frequency Domain Slant-stacking Algorithm}

The implementation of the frequency domain slant stacking method is based on the Seismic Unix Tools.
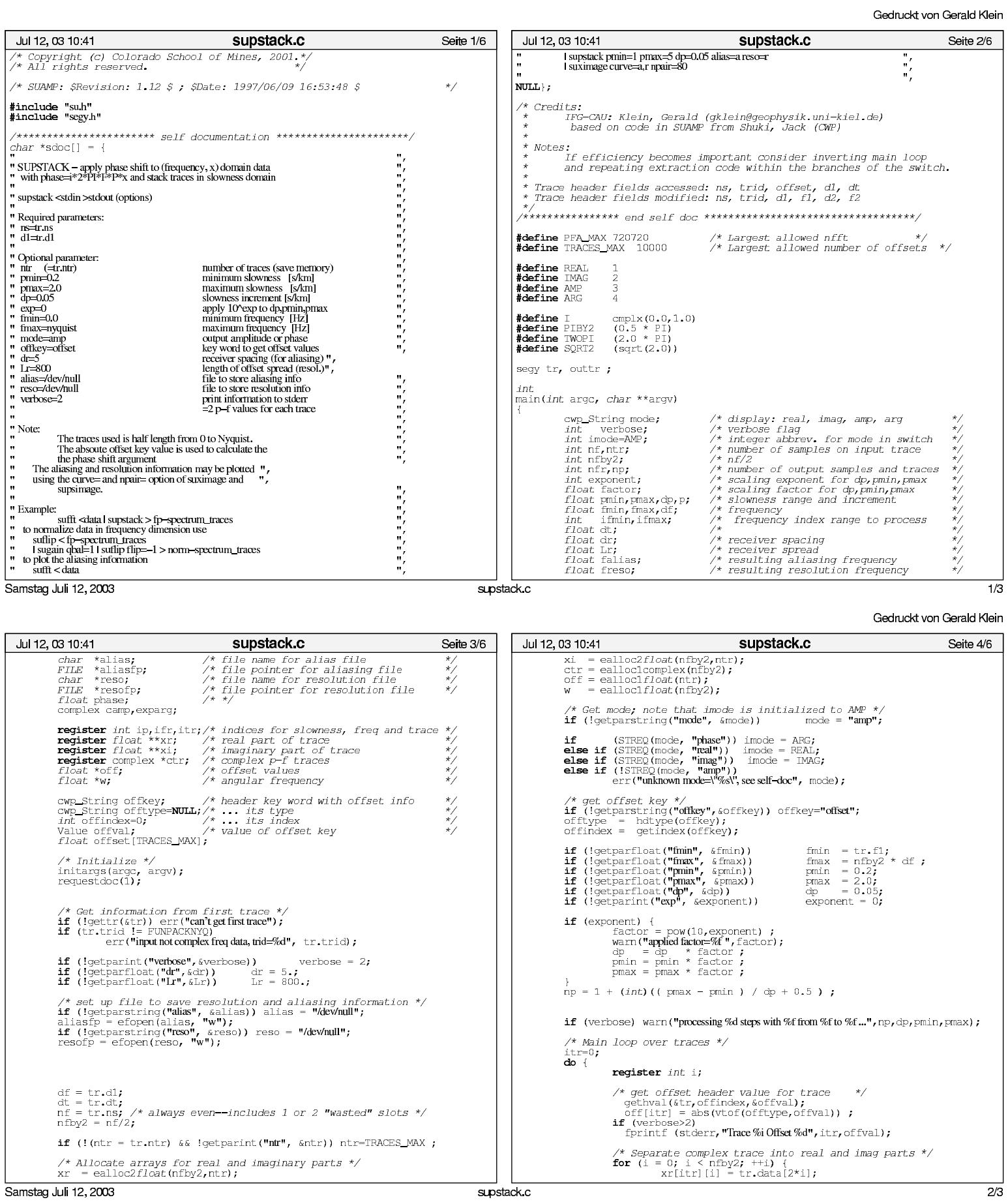


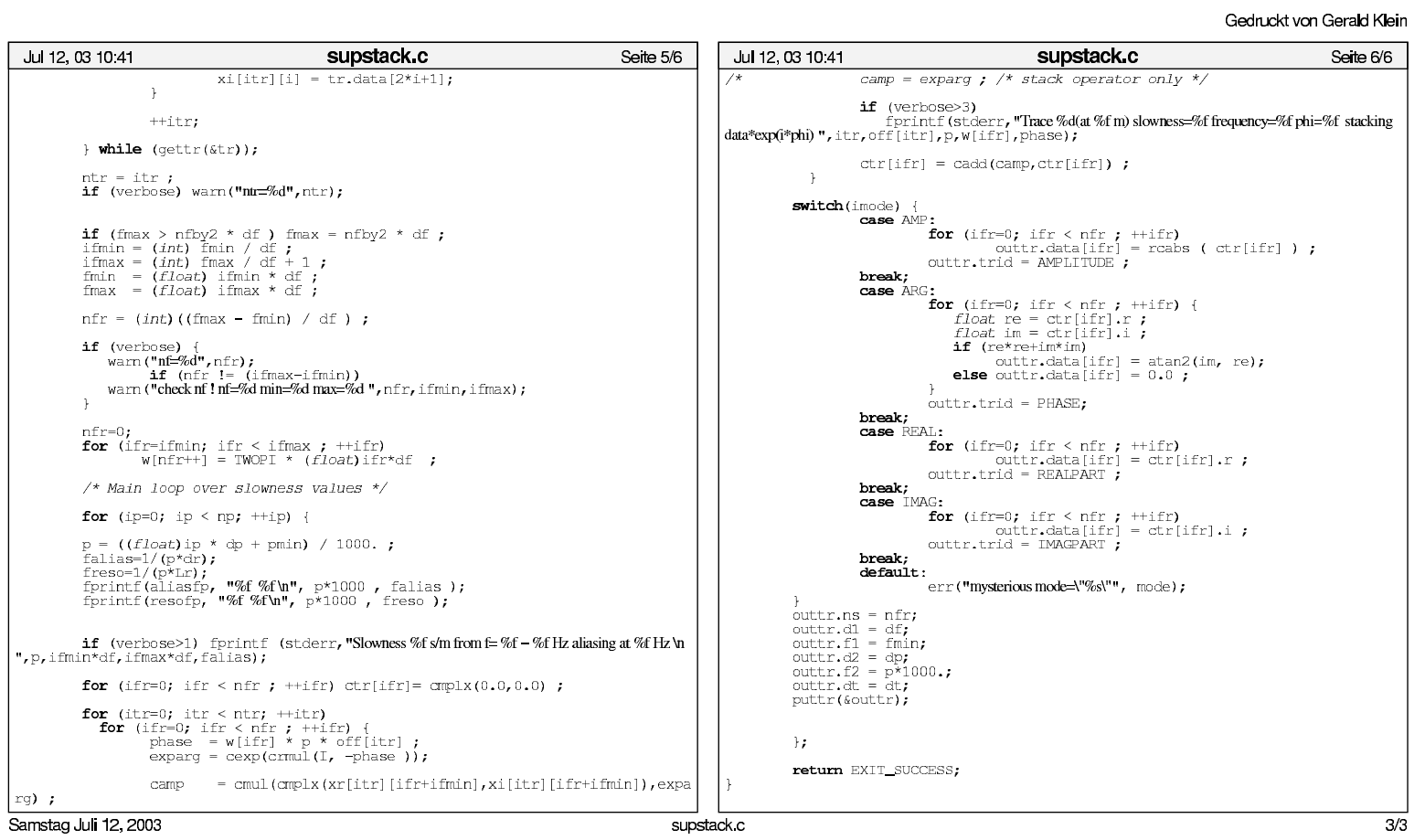

\section{D.2.3 Dispersion Curve Inversion and Visulization}

The dispersion curve inversion is performed in MATLAB with use of the Optimazation Toolkit(Grace, 1994). Visulization of synthetic wavefield spectra presented in the sensitivity study of Laptev Sea case study and in appendix C.1 was performed with MATLAB.

\section{D.2.4 Inversion of full Wavefield Spectra}

The preparation was performed using software STUFI, FIDASE and REFRACT of the StuttgartFileFormat package. The inversion was performed with WGREMLIN, all programs provided by Thomas Forbriger in personal communication.

\section{D.2.5 Other Tools}

The compilation of text, programs and data was performed on Linux based PCs and the SUN Solaris 2 server CORE with frequent use of standard shell scripting with make,awk, sed, and perl and other programs all under the GNU-Licence of the Free Software Foundation ${ }^{1}$. Images were produced with SU, GMT, GLE, GnuPlot and further processed using GIMP, ImageMagic and XFig programs. The typesetting was performed using Lyx and $\mathrm{LT}_{\mathrm{E}} \mathrm{X}$.

\footnotetext{
${ }^{1}$ http://www.gnu.de
} 


\section{D.3 List of Abbrevations and Symbols}

This list of abbrevations and symbols is a collection of terms and symbols frequently used throughout the thesis. Other terms used just in single occasions are explained in the text.

\section{Abbrevations}

OBS Ocean Bottom Seismometer

OBH Ocean Bottom Hydrophone

OBC Ocean Bottom Cable

CRG Common-receiver-gather

MFT Multiple frequency technique

SASW Spectral Analysis of Surface Waves

AGC Automatic Gain Control amplitude scaling

TWT Two-Way-Traveltime

\section{Symbols}

$x, r \quad$ distance or offset variable in cartesian and spherical coordinates

$t$ time

$f, \omega \quad$ frequency and angular frequency, $\omega=2 \pi f$

$\alpha, \beta \quad$ compressional and shear wave velocity of a medium

$c \quad$ phase velocity

$p \quad$ phase slowness, $1 / c$

$U$ group velocity

$\vec{u}(x, t)$ displacement vector in the time-distance domain

$u, w, v \quad$ displacement components of $\vec{u}$

$\vec{u}(\omega, p)$ displacement vector in frequency-slowness domain

$G(\omega p) \quad$ spectral coefficient 


\section{Acknowledgements}

This work would not have been possible without the contributions and help of many colleagues and friends. First of all I origin and foundation of these studies were encouraged and made possible by Prof. Milkereit and Prof. Rabbel. Also I wish to thank Thomas Bohlen and Friedrich Theilen for the guiding within the projects and field experiments.

For inspriring and motivating discussions I express my thank to Thomas Bohlen who supervised and guided my work with encouragement.

Many thanks are due to Thomas Forbriger as well as to Wolfgang Friederich, who not only donated the large program library for seismic data analysis and inversion but further gave inspiring discussions and good help in theoretical and computational aspects. Thanks for the kind hospitality and Stuttgart and Frankfurt University during my visits.

Simone Kugler I thank for numerous discussions and I would like to acknowledge the possibility to use some images from her diploma thesis and the jointly edited reports and paper.

The regular and fruitfull discussions with Thomas, Simone and Sascha gave inspiring thoughts as well as they provided good working atmosphere including the consumption of many liters of tea, thanks for that.

For the successful conduction of the many research cruises on RV Poseidon, RV Alkor, FK Littorina and FB Polarfuchs I owe my gratitude to the captains and crews, the senior scientists as well as the numerous student helpers and technicians without whom these marine seismic experiments would not have been possible.

For the reading of the manuscript I thank Inga Eisenberg, Monika Klein and Thomas Bohlen, also thanks to Thomas Forbriger for his comments on the selected chapters discussing the inversion methods.

I thank my family for support and encouragement and last but certainly not least, I express my most sincere thank to Inga Eisenberg for encouragement and patience in the long period of hard work.

Financial support was granted from the BGR, DFG and BMBF-Geotechnologien by funding the projects (BGR: Permafrost-98/99, DFG: SCHERSEIS no. Bo1721/1-1 and BMBF: INGGAS-NATLAB contract no. 03 G 0564 D). 


\section{Lebenslauf}

$\begin{array}{ll}\text { Name: } & \text { Gerald Klein } \\ & \text { Von-der-Tann-Str. } 7 \\ \text { Adresse: } & \text { 24114 KIEL } \\ \text { geboren: } & \begin{array}{l}\text { 17. Januar 1972, in Frankfurt am Main } \\ \text { Staatsangehrigkeit: }\end{array} \\ \text { deutsch }\end{array}$

Grundschule:

1976 - 1982 Römerstadtschule, Frankfurt am Main

Gymnasium:

1982 -1991 Wöhlerschule, Frankfurt am Main

Hochschulreife 1991

Studium:

1993 - 1995 Johann Wolfgang von Goethe Universtitt, Frankfurt Studienfach: Geophysik (Vordiplom 1995)

1995 - 2003 Christian-Albrechts-Univesitt zu Kiel

Hautpfach: Geophysik

Urlaubssemester WS 97/98 - WS 98/99

1997 - 1998 University of Wales, Bangor, UK

Abschluß: Master of Science Marine Geotechnics

School of Ocean Sciences, Menai Bridge

1999 - 2003 Christian-Albrechts-Univesitt zu Kiel

Wissenschaftlicher Angestellter,

Promotion 


\section{Eidesstattliche Erklärung}

Hiermit erkläre ich an Eides statt, dass die vorliegende Abhandlung, abgesehen von der Beratung durch meine Betreuer, nach Inhalt und Form meine eigene Arbeit darstellt und weder ganz noch zum Teil einer anderen Stelle im Rahmen eines Prüfungsverfahrens vorgelegen hat.

Teile der Arbeit sind in folgende Veröffentlichungen eingeflossen:

Bohlen T., Kugler S., Klein, G. \& Theilen, F.: 1.5-D Inverison of Lateral Variation of Scholte Wave Dispersion, submitted to Geophysics, 2003 